\title{
Immigranten \\ zwischen Einbürgerung und Abwanderung
}

Eine empirische Studie zur bindenden Wirkung von Sozialintegration

\author{
Dissertation \\ zur Erlangung des sozialwissenschaftlichen Doktorgrades der \\ Sozialwissenschaftlichen Fakultät \\ der Georg-August-Universität Göttingen
}

\author{
vorgelegt \\ von \\ Jürgen Leibold \\ aus Flieden
}

Göttingen 2006 
1. Gutachter: Prof. Dr. Steffen-Matthias Kühnel

2. Gutachter: Prof. Dr. Peter Schmidt

Tag der mündlichen Prüfung: 31.08.2006 


\section{Zusammenfassung}

Die Bundesrepublik Deutschland hat sich in den zurückliegenden Jahrzehnten durch Einwanderung von einer tendenziell ethnisch homogenen zu einer ethnisch heterogenen Gesellschaft gewandelt. Im Zuge der einzelnen Einwanderungsphasen sind von den Immigranten, ihren Nachkommen und der Aufnahmegesellschaft erhebliche Integrationsleistungen erbracht worden. Ein Teil der Menschen mit Migrationshintergrund entschied sich jedoch die Aufnahmegesellschaft zu verlassen, während andere für immer oder zumindest für sehr lange Zeit zu bleiben beabsichtigen. Welche Faktoren hierfür ausschlaggebend sind und wie die Prozesse aussehen, die zu dauerhafter Integration oder zu Abwanderung führen, ist Gegenstand der vorliegenden Arbeit. In enger Anlehnung an theoretische Arbeiten von Hartmut Esser wird ein Handlungsmodell zur Sozialintegration von Immigranten vorgestellt, mit dessen Hilfe das Auftreten bzw. Ausbleiben von Einbürgerung und Abwanderung erklärt werden soll. Auf Basis der Daten des Sozio-oekonomischen Panels wurde das entwickelte Modell einer empirischen Prüfung unterzogen, in deren Rahmen sich der Erwerb kultureller Kenntnisse und der Aufbau sozialer Kontakte zu den Angehörigen der Aufnahmegesellschaft erwartungsgemäß als bestimmend für die Sozialintegration der Personen mit Migrationshintergrund erwiesen. Entgegen der theoretischen Erwartung konnte für die Positionierung auf dem Arbeitsmarkt kein wesentlicher integrationsfördernder Effekt festgestellt werden. Darüber hinaus ergaben sich zwischen einzelnen Migrantengruppen erhebliche Unterschiede hinsichtlich der Bedeutung von Sozialintegration für die Erklärung von Abwanderungsund Einbürgerungsvorgängen. Während EU-Bürger trotz guter bis sehr guter Sozialintegration erhebliche Abwanderungsraten aufweisen und sich eher selten für Einbürgerungen entscheiden, tendieren Personen türkischer Herkunft bei wesentlich geringeren Integrationswerten weniger zur Abwanderung und eher zu dauerhaftem Aufenthalt und Einbürgerung. Die Befunde deuten daraufhin, dass dissimilative Komponenten der Sozialintegration, wie z.B. transnationale Bezüge und kulturelle Pluralisierung, für die Erklärung von Abwanderungs- und Einbürgerungsvorgängen von größerer Bedeutung sind. 


\section{Abstract}

Within the last few decades the Federal Republic of Germany has changed through immigration from a rather ethnically homogeneous, to an ethnically heterogeneous society. In the course of the several immigration periods, since the nineteen fifties, considerable integration work has been undertaken by the immigrants, their descendants and the host society. Part of the people with a migration background, though, decided to leave the host country, while others intend to remain for ever or at least for a very long time. Which factors are decisive for this and what kind of processes lead to a durable integration or to re-migration are the topics of this dissertation. Using the theoretical work of Hartmut Esser, an individual action model is introduced, which seeks to explain the appearance or non-appearance of naturalisation and re-migration by the social integration of people with a migration background. Based on the data set of the German Socio-Economic Panel, the action model was subjected to an empirical examination. As expected, the examination showed that the acquisition of cultural knowledge and developing contacts to members of the host society, are factors that determine social integration. Contrary to the theoretical expectation, the position on the labour market plays no essential role in the social integration of immigrants and their descendants. In addition, considerable differences appear between several groups of immigrants, particularly with regard to the effect of social integration on re-migration and naturalisation. Immigrants from member states of the European Union show higher re-migration rates and tend far less towards naturalisation although they show a high degree of social integration. On the other hand, individuals of Turkish origin are in comparison less integrated but tend rather more towards long-term stay and naturalisation. The results indicate that dissimilative components of social integration, such as transnational ties and cultural pluralisation, are more important for the explanation of naturalisation and re-migration of immigrants. 


\section{Inhalt}

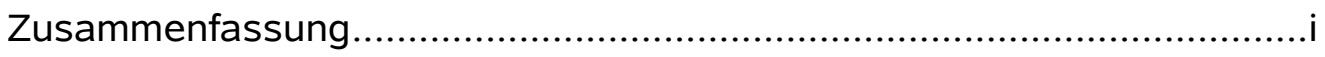

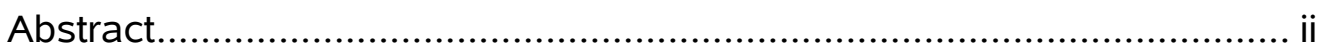

Verzeichnis der Abbildungen.............................................................. vi

Verzeichnis der Tabellen......................................................................vii

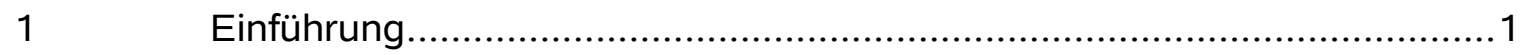

$1.1 \quad$ Fragestellung und thematische Eingrenzung ................................... 3

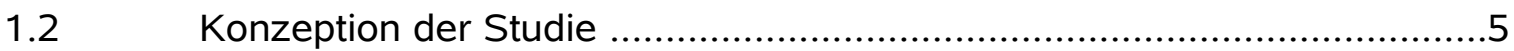

2 Forschungslinien $\mathrm{zu}$ den Anpassungsstrategien und Integrationsprozessen von Immigranten.............................................................. 7

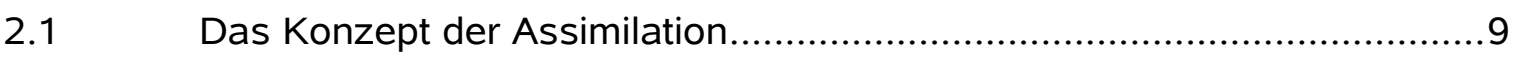

2.1.1 Phasenmodelle der Eingliederung.................................................

2.1.1.1 Ökonomische Modelle zum Integrationsverlauf................................... 10

2.1.1.2 Am Sukzessionsprozess orientierte Modelle.....................................11

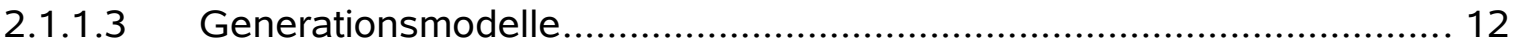

2.1.1.4 Race-Relation-Cycle ................................................................ 13

2.1.2 Determinanten der Assimilation.......................................................... 14

2.2 Ausschluss als ein Grundmuster sozialer Integration.......................... 19

2.3 Kulturelle Pluralisierung und transnationale Bezüge ...........................23

2.3.1 Kulturelle Pluralisierung von Einwanderungsgesellschaften..................23

2.3.2 Transnationale Bezüge der kulturellen Pluralisierung...........................27

2.4 Zusammenfassung und Zwischenfazit ............................................ 29

3 Abwanderung und Einbürgerung: Stand der Forschung...................... 34

3.1 Abwanderung: Absichten und Determinanten................................... 34

3.1.1 Umfang der Abwanderung von Immigranten und ihrer Nachkommen und Defizite der Erfassung......................................................................36

3.1.2 Empirische Ergebnisse zur Abwanderung von Personen mit Migrationshintergrund.

3.1.2.1 Rückkehrabsichten der Immigranten in der Bundesrepublik

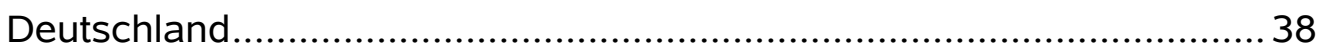

3.1.2.2 Determinanten des Abwanderungs- und Remigrationsverhaltens..........39 


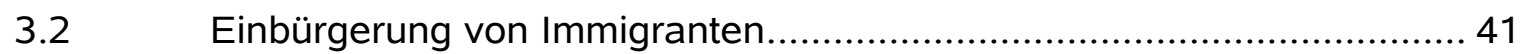

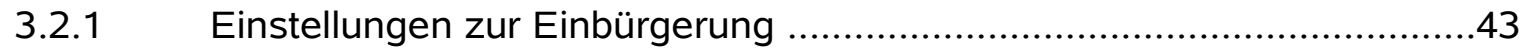

3.2.2 Determinanten der Einbürgerung ................................................ 45

3.2.2.1 Verwaltungshandeln und Einbürgerung in der Bundesrepublik Deutschland..................................................................................... 46

3.2.2.2 Determinanten der Einbürgerung nach Eigenschaften der

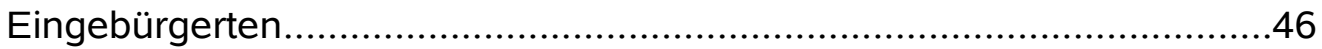

Z.3 Zwischenfazit zu den Forschungsergebnissen............................... 48

$4 \quad$ Ein handlungstheoretisches Modell zur Erklärung der Abwanderungsund Einbürgerungsvorgänge auf Basis des Niveaus der

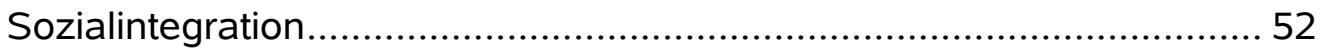

$4.1 \quad$ Integration: Von der Makro- zur Mikroebene.................................... 53

4.1.1 Sozialintegration und ihr Verhältnis zur Assimilation............................. 56

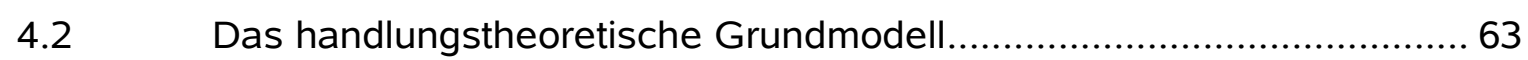

4.3 Handlungsentscheidungen vor dem Hintergrund der sozialen

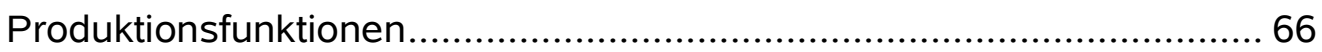

4.4 Individuelle Konsequenzen und die Summe der Handlungen............... 68

4.4.1 Entscheidungen zwischen Assimilation und Differenz ........................68 68

4.4.2 Die Aggregatebene: Veränderungen und deren Auswirkungen.............70

4.4.3 Abwanderung und Einbürgerung als Resultat der Sozialintegration.......75

4.5 Zusammenfassung und Diskussion.................................................78

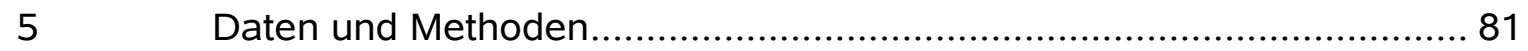

5.1 Das Sozio-oekonomische Panel des Deutschen Instituts für Wirtschaftsforschung: Konzeption und Design.

5.1.1 Teilnehmer des Sozio-oekonomischen Panels mit

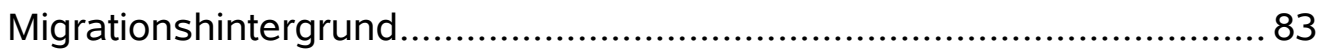

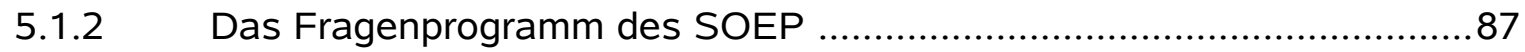

5.1.2.1 Operationalisierung der Konzepte des Kausalmodells......................... 89

5.1.3 Konsequenzen für die empirische Analyse.......................................... 99

5.2 Auswahl des statistischen Analysemodells zur Prüfung der kausalen Beziehungen an den Daten............................................................ 102

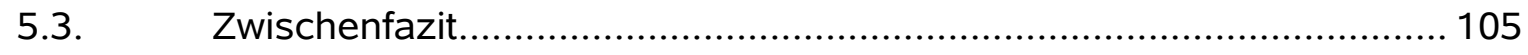


Analysen zur Erklärung der Abwanderungs- und

Einbürgerungsvorgänge.....

6.1 Sozialintegration der Personen mit Migrationshintergrund in den Daten des Sozio-oekonomischen Panels...................................................... 108

6.1.1 Die Entwicklung der Sozialintegration............................................... 111

6.1.2 Sozialintegration im Verhältnis zu den Abwanderungs- und Einbürgerungsvorgängen

6.1.3 Zwischenfazit zu den deskriptiven Ergebnissen.

6.2 Strukturgleichungsmodelle zur Erklärung der Abwanderungs- und Einbürgerungsvorgänge ............................................................125

6.2.1 Die kausale Struktur der Sozialintegration. 126

6.2.2 Sozialintegration als Erklärung für Einbürgerungs- und Abwanderungsvorgänge

6.2.3 Der Sozialintegrationsprozess als Erklärung für Einbürgerungs- und Abwanderungsvorgänge

6.2.4 Zusammenfassung zu den Ergebnissen der kausalen Analysen 144

Literatur. 156

Anhang - Personenfragebogen des Sozio-oekonomischen Panels der Welle 1999

Lebenslauf. 


\section{Verzeichnis der Abbildungen}

Abbildung 3.2.1a: Schema der Emigration-Remigrations-Schleifen mit zwei

Aufnahmegesellschaften.

Abbildung 3.3.1a: Zahl der Einbürgerungen von Ausländern in

Deutschland 1975 - 2004.

Abbildung 4.1a: Schemata zum Verhältnis von Systemintegration und

Sozialintegration in ethnisch homogenen und heterogenen

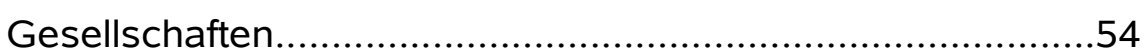

Abbildung 4.1.1a: Kausale Struktur der Assimilation nach Esser (2001)............60

Abbildung 4.1.1b: Prozessmodell der Assimilation nach Esser (1980)...............61

Abbildung 4.4.2a: Beispiele für Assimilation und Dissimilation der Aggregate....71

Abbildung 4.4.3a: Erklärungsmodell zur Einbürgerung und Abwanderung basierend auf dem Kern des Prozessmodells von 1980.........76

Abbildung 4.4.3b: Erklärungsmodell zur Einbürgerung und Abwanderung basierend auf dem Prozess der Sozialintegration.

Abbildung 6.1a: Anzahl der Personen in den Herkunftsgruppen nach Jahr der Erhebung. 110

Abbildung 6.2.1a: Struktur- und Messmodell der Sozialintegration.

Abbildung 6.2.1b: Modifiziertes Strukturmodell der Sozialintegration 1999.......128

Abbildung 6.2.2a: Struktur- und Messmodell zur Erklärung der Abwanderungsund Einbürgerungsvorgänge über die Sozialintegration.

Abbildung 6.2.2b: Strukturmodell zur Erklärung der Abwanderungs- und Einbürgerungsvorgänge zwischen 2000 und 2004 über das Niveau der Sozialintegration 1999.

Abbildung 6.2.3a: Struktur- und Messmodell zur Erklärung der Abwanderungsund Einbürgerungsvorgänge über den Prozess der Sozialintegration 140

Abbildung 6.2.3b: Strukturmodell zur Erklärung der Abwanderungs- und Einbürgerungsvorgänge über den Prozess der Sozialintegration. .142 


\section{Verzeichnis der Tabellen}

Tabelle 5.1.1a: Anzahl der Personen mit einer ausländischen

Staatsangehörigkeit aufgeschlüsselt nach Jahr der Erhebung und den einzelnen SOEP-Stichproben.

Tabelle 5.1.1b: Anzahl der Personen im SOEP, die nach 1948 in die

Bundesrepublik bzw. die Deutsche Demokratische

Republik zuwanderten.

Tabelle 5.1.1c: $\quad$ Anzahl der Immigranten im Vergleich zu Personen ohne deutsche Staatsangehörigkeit im SOEP nach

Erhebungsjahr. .86

Tabelle 5.1.2a: Übersicht zur thematischen Ausrichtung der Panelwellen des Sozio-oekonomischen Panels. .87

Tabelle 5.1.2b: Übersicht zu ausgewählten ausländerspezifischen Themenkomplexen im Sozio-oekonomischen Panel. 89

Tabelle 5.1.2.1a: Übersicht zu den ausgewählten Items und Variablen. .97

Tabelle 5.1.2.1b: Auswirkungen der Filterführung auf die Zusammensetzung der Antwortenden (1997-2004)

Tabelle 6.1.1a: Entwicklung der subjektiven Deutschkompetenz ausgewählter Migrantengruppen zwischen 1985 und 2003.

Tabelle 6.1.1b: Entwicklung der Sozialintegration über den Arbeitsmarkt nach ausgewählten Migrantengruppen zwischen 1985

und 2003

Tabelle 6.1.1c: Entwicklung der Sozialintegration ausgewählter Migrantengruppen über soziale Kontakte zu Deutschen zwischen 1985 und 2003 115

Tabelle 6.1.1d: Entwicklung der Sozialintegration ausgewählter Migrantengruppen über die emotionale Identifikation mit der Aufnahmegesellschaft zwischen 1985 und 2003. 116

Tabelle 6.1.2a: Umfang der Abwanderungs- und Einbürgerungsvorgänge ausgewählter Migrantengruppen zwischen 1985 und 2004..118 
Tabelle 6.1.2b: Entwicklung der Sozialintegration von später abgewanderten bzw. eingebürgerten Immigranten im Kontrast zu befragten Inländern ohne deutsche Staatsangehörigkeit von 1985 bis 2003

Tabelle 6.2.1a: Stärke der unstandardisierten Effekte und Güte der jeweiligen Modellanpassung des modifizierten Modells der Sozialintegration für die Jahrgänge 1985, 1989, 1995, 1999 und 2003. 129

Tabelle 6.2.1b: Stärke der unstandardisierten Effekte und Güte der jeweiligen Modellanpassung des modifizierten Modells der Sozialintegration getrennt nach Herkunftsgruppen für das Jahr 1999

Tabelle 6.2.2a: $\quad$ Stärke der unstandardisierten Effekte und Güte der jeweiligen Modellanpassung des Erklärungsmodells getrennt nach Herkunftsgruppen für das Jahr 1999. 137 


\section{Einführung}

Die dauerhafte oder auch nur vorübergehende Integration von Immigranten ist bedingt durch die weltweit zunehmenden Wanderungsbewegungen für viele Aufnahmegesellschaften zu einer wichtigen und fortwährenden Aufgabe geworden. Mit der Ankunft in einer Aufnahmegesellschaft beginnt in Abhängigkeit von den Eigenschaften und Absichten des jeweiligen Immigranten sowie den vorgefundenen Möglichkeiten ein Prozess, der ganz allgemein als wechselseitige Anpassung an die durch Einwanderung veränderten Lebensumstände bezeichnet werden kann. Welche Verhaltensmuster sich bei den beteiligten Akteuren und Gruppen herausbilden, ist von vielen Faktoren abhängig. In dieser Adaptationsphase sind Veränderungen der Fähigkeiten, Intentionen und Gefühle der Immigranten, wie auch der Bedingung in der Aufnahmegesellschaft nicht nur möglich sondern oft unumgänglich.

Auch die Bundesrepublik Deutschland bildet in dieser Hinsicht keine Ausnahme, denn seit dem Ende des Zweiten Weltkrieges sind aus unterschiedlichsten Motiven Menschen nach Deutschland gekommen. Viele von denen, die einreisten, kehrten in die alte Heimat zurück oder suchten sich neue Ziele und zogen weiter. Eine nicht unbeträchtliche Zahl an Menschen entschied sich dafür längerfristig oder für immer zu bleiben. ${ }^{1}$ Die Bundesrepublik Deutschland hat sich dadurch von einer eher ethnisch homogenen zu einer ethnisch heterogenen Gesellschaft gewandelt. Der Anteil der Personen mit Migrationshintergrund, die gleichzeitig nicht ethnisch-deutsch sind, wird mit etwa zwölf Prozent angegeben. Im Rahmen dieser Veränderungen sind erhebliche Integrationsleistungen von den Immigranten und der deutschen Gesellschaft erbracht worden, aber die Integration großer Teile der Bevölkerung mit Migrationshintergrund kann weder als abgeschlossen angesehen werden, noch besteht bei allen Zuwanderern und ihren Nachkommen ein Interesse daran, sich dauerhaft in die Aufnahmegesellschaft zu integrieren. So bestehen Bildungsunterschiede zwischen Deutschen und den Arbeitsmigranten, die sich zum Teil in den letzten Jahren verschärft haben. Ausländer sind allgemein in hohem Maße von Arbeitslosigkeit betroffen, es haben sich in vielen Großstädten ethnisch segregierte

1 Im Zeitraum von 1954 bis 2000 ist insgesamt ein Wanderungsgewinn von rund 9 Millionen Menschen zu verzeichnen gewesen, dabei stehen ca. 32 Millionen Zuzügen etwa 23 Millionen Fortzügen gegenüber. Der Ausländeranteil für die genannten Werte liegt ungefähr bei $80 \%$. (eigene Berechnung, Datenquelle: Statistisches Bundesamt) 
Wohnviertel gebildet und die Einstellung eines nennenswerten Teils der autochthonen Bevölkerung ist weiterhin von Distanz, Vorurteilen und Ablehnung gegenüber Immigranten gekennzeichnet.

Neben der ethnischen Heterogenisierung hatte die Zuwanderung auch erheblichen Einfluss auf die Bevölkerungsentwicklung der Bundesrepublik. Seit dem Ende der sechziger Jahre wurde der einsetzende natürliche Bevölkerungsrückgang durch die Zuwanderungsgewinne überkompensiert und das Verhältnis von älteren und jüngeren Menschen hätte sich ohne Immigration noch stärker in Richtung der Älteren verschoben. Für die Zukunft prognostiziert das Statistische Bundesamt, bedingt durch anhaltend niedrige Geburtenzahlen, einen Rückgang der Bevölkerungszahl, der, selbst bei erheblich verstärkter Zuwanderung, nicht mehr auszugleichen sein wird (Statistisches Bundesamt 2000). Jedoch lassen sich die mit den demographischen Veränderungen einhergehenden Folgen über Zuwanderung abschwächen und verzögern, was aber zusätzliche Integrationsleistungen erforderlich machen würde. Aus den Gutachten und Expertisen die für die Unabhängige Kommission Zuwanderung und den Sachverständigenrat für Zuwanderung und Integration erstellt wurden, geht hervor, dass die positiven Effekte der Zuwanderung die damit verbundenen Kosten übersteigen werden, wenn eine gezielte Auswahl im Hinblick auf das Integrationspotential der Immigranten getroffen wird (Eitenmüller/Gramke/Jens 2001, Schäfer 2004, Löffelholz/Bauer/HaiskenDeNew/Schmidt 2004, Brückner/Kohlhaas 2004). Ein Potential, das nur dann voll entwickelt werden kann, wenn gezielt integrationsfördernde Maßnahmen eingesetzt werden. In Bezug auf die Integrationsförderung von ethnisch-nichtdeutschen Immigranten in der Bundesrepublik kommt der Sachverständigenrat jedoch zu dem vernichtenden Urteil, es sei eine „Ansammlung von bereichsspezifischen, föderal zergliederten und weitgehend unkoordinierten, auf unterschiedlichen Ebenen und in unterschiedlichen Zuständigkeiten angesiedelten Konzepten. Es fehlte eine übergreifende strategische Ausrichtung, aus der sich Ziele und Maßnahmen der dezentral handelnden Akteure ableiten konnten. (...) nicht selten mangelte es den Konzepten auch an der Berücksichtigung von Zusammenhängen zwischen den verschiedenen Bereichen und mithin an Kohärenz, Effektivität und Zielgenauigkeit.“ (Sachverständigenrat für Zuwanderung und Integration 2004, 433-434). 
Um diesen Defiziten Rechnung zu tragen, fordern die Sachverständigen unter anderem eine intensivere Erforschung der Integrationspotentiale und -prozesse, denn sie sehen die Migrations- und Integrationspolitik bislang aufgrund fehlender Informationen im „Blindflug“ (vgl. Sachverständigenrat für Zuwanderung und Integration 2004, 415-435).

Allerdings steht die sozialwissenschaftliche Forschung hinsichtlich der Anpassungsstrategien und Integrationsprozesse - wie für die Sozialwissenschaften eher typisch - noch am Anfang, was nicht zuletzt daran liegt, dass es sich um komplexe Interdependenzen handelt, die wiederum im Zusammenhang mit der jeweiligen Migrations- und Integrationspolitik stehen. Um Antworten auf die drängenden Fragen zur Integration geben zu können, genügt es nicht, die wesentlichen Dimensionen der Sozialintegration von Immigranten zu klären, sondern es ist auch notwendig, die kausalen Zusammenhänge zwischen ihnen zu analysieren. Solange aber die kausale Struktur der Integrationsprozesse nicht hinreichend geklärt ist, lassen sich die Effekte von integrationspolitischen Maßnahmen nur schwerlich prognostizieren und evaluieren. Damit bleibt aber zunächst offen, wie eine auf Dauer und Nachhaltigkeit angelegte Integrationspolitik aussehen sollte, damit die vorhandenen Integrationspotentiale effektiv entwickelt werden können. Aber diese Frage ist von zentraler Bedeutung, denn die Lebensverhältnisse der Immigranten und die Entwicklung der aufnehmenden Gesellschaft werden davon erheblich mitgeprägt.

\subsection{Fragestellung und thematische Eingrenzung}

Im Kontext der hier angesprochenen migrations- und integrationspolitischen Erfordernisse ergeben sich aber noch eine Reihe weiterer Fragen. Soll der Integrationsprozess in seiner Gesamtheit erfasst werden, dann stehen zu Beginn zwei Fragen: Welche Voraussetzungen bringen die Immigranten mit und welche sind in der Aufnahmegesellschaft gegeben? Davon ausgehend sind die Entwicklungsverläufe von besonderer Bedeutung: Worauf sind die erheblichen Unterschiede in den Integrationsbemühungen und -erfolgen zurückzuführen? Welche Bedeutung besitzen die Einstellungen und Fähigkeiten der Einwanderer und welche die Restriktionen bzw. Angebote der Aufnahmegesellschaft im Hinblick auf den Integrationsverlauf? Las- 
sen sich Muster in den wechselseitigen Reaktionen erkennen oder entziehen sich diese weitgehend einer Systematisierung? Ist es wesentlich, Bereiche der Integration zu unterscheiden und wenn ja, welche sind wichtig und welche Beziehungen bestehen zwischen diesen? Wie ist es um das Verhältnis von Integration und Aufenthaltsabsichten bestellt? Gute Integration in einzelnen Teilbereichen ist nicht unbedingt an eine dauerhafte Aufenthaltsabsicht gekoppelt, denn nicht jeder Immigrant, der eine beruflich hohe Position einnimmt und dadurch viele Kontakte zu Mitgliedern der Aufnahmegesellschaft unterhält, muss zwangsläufig für immer im Aufnahmeland bleiben wollen. Daraus ergibt sich die Frage: Wie sehen die Integrationsverläufe aus, die dazu führen, dass Immigranten und ihre Nachkommen sich entscheiden, für immer zu bleiben, während andere beabsichtigen, das Aufnahmeland zu verlassen?

Nicht alle genannten Fragen sind im Rahmen dieser Arbeit zu beantworten, aber ausgehend von der letzten Frage ist es das Ziel über die Integration in eine Aufnahmegesellschaft die Abwanderung, den Aufenthalt sowie die Einbürgerung von Immigranten zu erklären. Dabei ist es unumgänglich, auch auf die für das Aufenthaltsverhalten der Immigranten bedeutsamen Integrationsaspekte einzugehen. Dem Erklärungsversuch wird das handlungstheoretische fundierte Modell der Sozialintegration von Hartmut Esser (Esser 1980, 2001, 2003) zugrunde gelegt. Die Theorie Essers zur Eingliederung von Immigranten hat in der deutschsprachigen Migrationsund Integrationsforschung viel Aufmerksamkeit gefunden und zählt hierzulande zu den einflussreichsten theoretischen Arbeiten im Bereich der Integrationsforschung (Nauck 1988, Angenendt 1992, Heckmann 1992; Treibel 2003, Han 2000). Zur Modellierung der Integration verwendet Esser ein aus vier Subdimensionen bestehendes Kausalmodell der Assimilation, das theoretisch an die Arbeiten von Shmuel N. Eisenstadt, Milton M. Gordon und Ronald Taft zu den Determinanten der Eingliederung von Immigranten anknüpft. (vgl. Esser 1980, 70). Die assimilationstheoretischen Ansätze und insbesondere das Modell von Esser erscheinen mir für den Erklärungsversuch besonders geeignet, weil die Assimilationsforschung sich der Erforschung von Anpassungsstrategien und Integrationsprozessen widmet und dabei das Gelingen oder Misslingen eines fortschreitenden, typischerweise intergenerativen Prozesses der sozioökonomischen, kulturellen und sozialen Integration in die Aufnahmegesellschaft ins Zentrum des Interesses rückt (vgl. Alba/Nee 2004). 


\subsection{Konzeption der Studie}

Integration von Immigranten und ihren Nachkommen in der von immer stärkerer Mobilität geprägten Welt ist in den letzten Jahren stärker in den Fokus des sozialwissenschaftlichen Forschungsinteresses gelangt. Es werden nicht nur in den klassischen Einwanderungsnationen, sondern auch im europäischen Raum zunehmend neue Publikationen zu diesem Thema veröffentlicht. Das Spektrum an unterschiedlichen Interpretationslinien und Erklärungsansätzen nimmt dadurch ständig weiter zu. Auf der Basis einer von Richard Alba und Victor Nee vorgelegten Klassifizierung soll im anschließenden Kapitel (2) ein Überblick zu wesentlichen Forschungslinien der sozialwissenschaftlichen Auseinandersetzung mit dem Thema Immigration und Integration gegeben werden. Es geht bei dieser Übersicht in erster Linie darum, die wesentlichen theoretischen Differenzen herauszuarbeiten und die Ansätze dann hinsichtlich der Verwendbarkeit als theoretische Basis für die gewählte Fragestellung einzuordnen.

Nach der Einführung in die Hauptlinien der Integrationsforschung werden in Kapitel 3 entsprechend der in der Forschungsliteratur vorherrschenden Trennung von Remigration/Abwanderung auf der einen und Einbürgerung auf der anderen Seite die Ergebnisse wichtiger Forschungsarbeiten zu beiden Themenbereichen referiert. Der Schwerpunkt wird auf den Determinanten der Remigrationsabsicht und des Abwanderungsverhaltens liegen, die eine Reihe von Parallelen zu den bestimmenden Faktoren der Einbürgerungsabsicht und der Naturalisierungsvorgänge aufweisen. Mit Bezug auf diese Gemeinsamkeiten in den Forschungsergebnissen soll verdeutlicht werden, warum eine Interpretation der Abwanderung und Einbürgerung im Sinne von Eckpunkten der Integration als angemessen angesehen werden kann.

Vor diesem Hintergrund wird der theoretische Bezugsrahmen für die Erklärung von Einbürgerungs- und Abwanderungsvorgängen in Kapitel 4 dargestellt. Es handelt sich dabei um den von Esser vorgestellten und in der Tradition der Assimilationsforschung stehenden handlungstheoretischen Ansatz zur Sozialintegration von Immigranten und ihren Nachkommen. Der Ansatz wird dazu nicht einseitig im Sinne einer auf Assimilation bezogenen Entwicklung, sondern im Sinne eines ergebnisoffenen Assimilations-Dissimilations-Prozesses interpretiert, an dessen Ende Einbür- 
gerung und Abwanderung als Resultat der unterschiedlichen Integrationsverläufe stehen können. In diesem Zusammenhang werden auch mögliche Rückwirkungen im Hinblick auf die Zusammensetzung der ethnischen Gruppen und das Konfliktpotential zwischen ethnischen Gruppen einer Aufnahmegesellschaft diskutiert. Aufbauend auf den theoretischen Vorarbeiten wird am Ende ein Prozessmodell zur Erklärung des Auftretens bzw. Ausbleibens von Einbürgerung und Abwanderung vorgestellt, das als Grundlage für die statistischen Analysen dienen wird.

Ausgehend von den theoretischen Vorgaben wird im folgenden fünften Kapitel die Auswahl des Sozio-oekonomischen Panels als Datenbasis für die Analysen erläutert und ein Überblick zu den im Fragenprogramm vorhandenen Informationen gegeben. Unter Berücksichtigung der kausalen Struktur des Erklärungsansatzes und der Zusammensetzung der Daten werden im Anschluss daran in Frage kommende statistische Analysemodelle diskutiert.

Das im vierten Kapitel entwickelte Prozessmodell der Sozialintegration wird in Kapitel 6 zur Erklärung der Einbürgerungs-Abwanderungs-Vorgänge anhand der SOEP-Daten eingesetzt. Zur Einführung in die Daten wird zunächst ein deskriptiver Überblick zu den Beziehungen der einzelnen Bestandteile des Erklärungsmodells im Verlaufe der Jahre und im Vergleich von Herkunftsgruppen bzw. der Immigrantengenerationen gegeben. Im zweiten Teil des Kapitels steht dann der schrittweise Test des an der Sozialintegration orientierten Erklärungsmodells im Mittelpunkt. Im Zuge dieser Prüfung an den empirischen Daten wird es auch um die Stabilität der kausalen Beziehungen innerhalb des Modells der Sozialintegration und im Hinblick auf die zu erklärenden Vorgänge gehen, wenn unterschiedliche Zeitpunkte und Herkunftsgruppen getrennt betrachtet werden. Das Schlusskapitel (7) ist auf eine Einordnung der Ergebnisse im Rahmen der Integrationsforschung mit Bezug zu den einleitend aufgeworfenen migrations- und integrationspolitischen Fragen ausgerichtet. 


\section{Forschungslinien zu den Anpassungsstrategien und Integrationsprozessen von Immigranten}

Mit den Integrationsverläufen und den Anpassungsstrategien von Immigranten beschäftigt sich die sozialwissenschaftliche Forschung nicht erst seit Integration von Einwanderern und ihrer Nachkommen in Deutschland einen oberen Platz auf der politischen Agenda einnimmt. Bereits Klassiker der Sozialwissenschaften widmeten dem Forschungsbereich Migration und Integration ihre Aufmerksamkeit. Neben den Arbeiten der Chicago-School sind hier vor allem die von Georg Simmel und Alfred Schütz über den „Fremden“ und Max Webers Ausführungen zu ethnischen Gruppen zu nennen (Simmel 1908, Schütz 1944, Weber 1922). Aber nicht nur soziologisch orientierte Autoren, sondern auch Vertreter der Ökonomie, Sozialpsychologie und nicht zuletzt Politik- und Geschichtswissenschaft haben sich inhaltlich und theoretisch mit der Integration von Einwanderern auseinandergesetzt und Wesentliches zu unserem heutigen Verständnis beigetragen.

Gegenwärtig bezieht die wissenschaftliche Auseinandersetzung mit der Integration von Immigranten ihre gesellschaftliche Relevanz über mehrere Problemfelder. An erster Stelle sind hier die Folgen der Einwanderungsschübe in den zurückliegenden Jahrzehnten zu nennen, die zu einer ethnischen, religiösen und kulturellen Pluralisierung geführt haben, auf der zum Teil nicht unerhebliche Segmentationen und Stratifikationen in den einzelnen Gesellschaften basieren. Ein zweiter Beweggrund für die Auseinandersetzung mit der Eingliederung von Immigranten ist in dem weiterhin bestehenden Immigrationsdruck in Richtung Europa und Nordamerika zu sehen. Gleichzeitig verbreitet sich in den europäischen Zuwanderungsgesellschaften, die von einem mehr oder weniger allmählichen Bevölkerungsrückgang betroffen sind, die Ansicht, dass prognostizierte demographische Veränderungen und die daraus resultierenden Konsequenzen mit einer darauf abgestimmten Einwanderung verzögert und abgeschwächt werden können.

Im Mittelpunkt des sozialwissenschaftlichen Interesses stehen deshalb die Integrationsverhältnisse (vgl. Bade/Bommes 2004, S7). Das Forschungsfeld ist stark ausdifferenziert und von ganz unterschiedlichen thematischen, theoretischen und normativen Schwerpunktsetzungen gekennzeichnet. Beschreibungen und Analysen 
der Integrationsverhältnisse sind mit einer Vielzahl an Begriffen und Bildern ${ }^{2}$ verbunden, die eine Systematisierung durch variierende Bedeutungsinhalte zusätzlich erschweren. Darüber hinaus bestehen bei einem großen Teil der wissenschaftlichen Veröffentlichungen sowohl explizit als auch implizit enge Verbindungen zwischen Darstellungsformen bzw. Erklärungsansätzen auf der einen und politischen Programmen auf der anderen Seite. Diese Verbindung von empirischen Aussagen und normativen Grundlagen der jeweiligen theoretischen Ausrichtung lässt es daher sinnvoll erscheinen, auch die zum Teil implizit transportierten normativen Aspekte zu benennen, zumal sie oft unmittelbar auf politische Integrationskonzepte ausgerichtet sind.

Günter Endruweit legte 1975 eine Bestandsaufnahme zur theoretischen Entwicklung der Integrationsforschung vor, in der er zu der Feststellung gelangte, dass die Eingliederungsforschung von einem Nebeneinander unterschiedlichster Ansätze gekennzeichnet ist (vgl. Endruweit 1975, 225). Richard Alba und Victor Nee haben eine Klassifizierung vorgestellt, die auf der Beschreibung und Erklärung der sozialen Integration von Einwanderern und ihrer Nachfahren in Nordamerika beruht, dieser folgend soll die wissenschaftliche Auseinandersetzung mit den Integrationsverhältnissen und Anpassungsstrategien dargestellt werden. Gemäß dieser Klassifizierung werden drei grundlegende Konzepte der Integrationsforschung unterschieden, die in der Vergangenheit Bedeutung erlangt haben und voraussichtlich auch weiterhin besitzen werden: Die Forschungslinien lassen sich unter den Begriffen Assimilation (2.1), rassistischer Ausschluss (2.2) und kulturelle Pluralisierung (2.3) zusammenfassen (vgl. Alba/Nee 2004). In den nun folgenden Abschnitten sollen die-se drei Hauptlinien der wissenschaftlichen Auseinandersetzung mit den Integrationsverläufen und Anpassungsmustern von Immigranten skizziert werden, um darauf aufbauend Konsequenzen für die theoretische Modellierung des Untersuchungsvorhabens abzuleiten. Der Schwerpunkt liegt dabei nicht auf empirischen Ergebnissen, sondern auf den theoretischen Entwicklungen und grundsätzlichen Perspektiven der wissenschaftlichen Auseinandersetzung, die eingebettet in die normativen Positionen und politischen Programmatiken dargestellt werden sollen.

2 z.B.: Integration, Assimilation, Inkorporation, Absorption, Akkomodation, Inklusion/Exklusion, Pluralisierung, Amalgamation, Adaptation, Akkulturation, melting-pot, salad bowl, anglo-confromity, 


\subsection{Das Konzept der Assimilation}

Unter Assimilation wird ganz allgemein ein Anpassungsmuster verstanden, bei dem Merkmale eines Individuums oder einer Gemeinschaft denen einer Referenzgruppe ähnlicher werden (vgl. Brubaker 2001). Diese Angleichung geht, im Kontext von Migration hauptsächlich von den Immigranten aus und meist in sehr viel geringerem Umfang von den autochthonen Mitgliedern der Aufnahmegesellschaft. Das Assimilationskonzept ist das älteste und über lange Zeit auch dominierende Konzept in der wissenschaftlichen Erforschung von Integrationsverläufen gewesen. Allerdings ist am Assimilationskonzept in den letzten Jahrzehnten immer heftig Kritik geübt worden. Die Kritik bezieht sich zum einen auf die zum Teil normativen Interpretationen der Assimilation und zum anderen auf Integrationsverläufe, die nicht im Sinne einer Angleichung, sondern nur im Hinblick auf Stabilisierung und Entwicklung von Differenz interpretiert werden können.

\subsubsection{Phasenmodelle der Eingliederung}

In den Sozialwissenschaften wurde die Assimilation von Immigranten sehr früh zu einem zentralen Thema (vgl. Han 2000, S.40). Parallel zur Ausdifferenzierung der Sozialwissenschaften in der ersten Hälfte der 20. Jh. etablierte sich das, was heute im Nachhinein als Assimilationsforschung bezeichnet werden kann. So erschienen bereits 1914 „Racial Assimilation in Secondary Groups“ von Park und 1918 die ersten beiden Bände von „The Polisch Peasant in Europe and America“ von Thomas und Znaniecki (Park 1914; Thomas/Znaniecki 1918).

Im Rahmen der Erklärung von Anpassungsprozessen wurden in den Anfangsjahren eine Reihe von Modellen entwickelt, die weniger theoretisch als vielmehr deskriptiv einzelne Phasen des Eingliederungsprozesses unterscheiden. In der Regel wird dabei unterstellt, dass es unabhängig von den jeweiligen historischen Gegebenheiten, für die Eingliederung von Immigrantengruppen typische Phasen von feststehender Reihenfolge gibt, die letztendlich Assimilation zur Folge haben. In der Logik der Phasenmodelle kann dieser Prozess zwar durch unterschiedliche Faktoren verzögert aber nicht verhindert werden, auch eine Umkehrung des einmal eingeschlagenen Weges zur Assimilation oder das Auslassen einzelner Phasen, im 
Sinne einer partiellen Assimilation, war nur in wenigen Modellen vorgesehen (vgl. Price 1969).

In seinem Übersichtsartikel „The study of Assimilation“ unterscheidet Charles Price vier Typen von Phasenmodellen der Assimilation: die ökonomischen, ökologischen und generationsbezogenen Sequenzmodelle sowie die so genannten racerelation-cycles (vgl. Price, 1969, S. 200-217).

\subsubsection{1 Ökonomische Modelle zum Integrationsverlauf}

Die aus der Ökonomie stammenden Modelle beschreiben den Integrationsprozess in Abhängigkeit von Konjunkturzyklen der Aufnahmegesellschaft. Ausgangspunkt ist dabei eine wachsende Industrieproduktion, die einen erhöhten Arbeitskräftebedarf nach sich zieht. Wird dieser Bedarf in den unteren Lohnsektoren durch Einwanderer gedeckt, so die weitere Argumentation, dann entstehen durch den verschärften Wettbewerbsdruck Konflikte zwischen den Eingewanderten und Einheimischen. Im Rahmen der von den Modellen erwarteten zyklischen konjunkturellen Verschlechterung entsteht erhöhte Konkurrenz um Arbeitsplätze. Als Reaktion darauf verstärken sich die Konflikte und können bis hin zu gewalttätigen Auseinandersetzungen gehen. Auf politisch-administrativer Ebene kann in dieser Situation mit restriktiveren Einwanderungsbestimmungen reagiert werden, um weiteren Druck zu vermeiden. Verbessern sich die wirtschaftlichen Rahmenbedingungen wieder, reduziert sich das Konfliktpotential und damit sinkt die Ablehnung der Einwanderer durch die Mehrheitsgesellschaft. Angeregt durch den erneut ansteigenden Arbeitskräftebedarf wird die Einreise für Arbeitsmigranten wieder erleichtert. Im nächsten periodischen Abschwung werden erneut Konflikte ausgetragen, erreichen, bedingt durch die zunehmende Integration der ehemaligen Immigranten, aber nicht die Schärfe der vorhergehenden Periode (Price 1969).

Modelle dieses Typs erklären zwar das Entstehen von Vorurteilen, Diskriminierung und fremdenfeindlicher bzw. rassistischer Gewalt, machen darüber hinaus aber nur wenig bis keine Aussagen über die Integration von Immigranten. Inwieweit Einwanderergruppen sich im Zuge der vornehmlich ökonomischen Integration assi- 
milieren bleibt dabei offen. In neueren Arbeiten wird der Assimilation größere Bedeutung eingeräumt, so betrachtet Christian Dustmann beispielsweise die Aufenthaltsdauer im Zusammenhang mit den Investition in spezifisches Kapital des Aufnahmelandes (vgl. Dustmann 2002). In der Regel werden allerdings andere kausale Zusammenhänge untersucht, bei denen die ökonomische Integration als abhängige Variable der Assimilierung betrachtet wird (vgl. Pischke 1993, Chiswick 1978). Der Schwerpunkt der ökonomischen Migrationsforschung liegt aber weniger im Bereich der Assimilation und Integration als viel mehr im Bereich der Erklärung von Wanderungsverhalten (vgl. Haug 2000b).

\subsubsection{Am Sukzessionsprozess orientierte Modelle}

Zur Modellierung der Integration und Assimilation von Immigranten wurden im Rahmen der Stadtforschung an den ökologischen Sukzessionsprozess angelehnte Phasenmodelle entwickelt, in denen davon ausgegangen wird, dass auf Grundlage der spezifischen lokalen Voraussetzungen und den Eigenschaften der einwandernden Gruppe assimilative Prozesse ablaufen (Wirth 1928, Glazer 1957, Price 1964). Abstrahiert man von den spezifischen Besonderheiten, dann lassen sich aber auch einige Gemeinsamkeiten der Modelle erkennen: In der Pionierphase werden zunächst für die etablierte Bevölkerung weniger attraktive Wohngegenden durch die Immigranten besiedelt. Gleichzeitig findet eine ökonomische Integration auf der Ebene von gering bewerteten Berufspositionen statt. In dieser Phase werden auch Einrichtungen und Institutionen aufgebaut, die an die Herkunftskultur der Einwanderer anschließen. Die zweite Phase ist vom sozialen Aufstieg eines Teils der Ghettobewohner gekennzeichnet, die daraufhin in bessere Viertel umziehen und sich oft auch in ihrem Verhalten und ihren Einstellungen der Aufnahmegesellschaft gegenüber öffnen. Für die sich daran anschließenden Entwicklungen ist die Phaseneinteilung nicht einheitlich, aber die beschriebenen Prozesse sind nahezu identisch. Die freiwerdenden Nischen werden durch neue Immigranten besetzt. Aus der heterogeneren Ghettobevölkerung steigen im Verlauf der Zeit weiterhin Personen oder Familien auf und siedeln in besser ausgestattete Wohngebiete um. Mit der zunehmenden räumlichen Vermischung von autochthoner und allochthoner Bevölkerung 
sind auch zunehmend bikulturelle Freundschaftsbeziehungen verbunden und entsprechende Partnerschaften werden eingegangen, die letztlich zu einer Auflösung der Gruppengrenzen führen können. Mit der schrittweisen Reduzierung der Ghettogesellschaft auf einen Kern von Personen, die ihre Ethnizität stärker betonen, kann es aber auch zu entgegengesetzten Entwicklungen kommen, die sich in der stärkeren Betonung der Herkunftskultur und einem Ausbau der ethnischen Infrastruktur äußern. Alle diese Modelle unterstreichen die Bedeutung von räumlicher Konzentration einer Immigrantengruppe für den Aufbau einer ethnischen Kolonie und den weiteren Integrations- und Assimilationsprozess. ${ }^{3}$ Am Beispiel der amerikanischen Juden und ihrer Integration in die Gesellschaft entwarf Louis Wirth 1928 ein entsprechendes fünfstufiges Modell. Zu einer ähnlichen Phaseneinteilung gelangten Rex und Moore 1967 bei der Darstellung der Integration von Immigranten aus Irland, Pakistan und Indien in Birmingham. ${ }^{4}$

\subsubsection{Generationsmodelle}

Nahezu zeitgleich zu den ökologischen Modellen wurden Generationsmodelle entworfen (vgl. Price 1969 S. 204-213). Diese trugen dem Umstand Rechnung, dass eine umfassende Eingliederung in aller Regel nicht durch die Einwanderergeneration zu leisten ist, sondern erst durch nachfolgende Generationen erreicht werden. Demnach gliedert sich die Mehrzahl der ersten Generation nur partiell in die Aufnahmegesellschaft ein, der Schwerpunkt liegt dabei auf der ökonomischen Integration. Parallel wird der Aufbau ethnischer Netzwerke und Institutionen betrieben. Die zweite Generation ist von dem Spagat zwischen elterlicher Herkunftskultur und den Anforderungen durch die Aufnahmegesellschaft in Schule und Beruf geprägt. In dieser Brückengeneration steigt der Anteil bikultureller Freundschaften und Ehen. Erst die dritte Generation ist den Modellen zufolge in der Lage, die Herkunftskultur gänzlich aufzugeben und sich als Teil der Aufnahmegesellschaft zu verstehen. Das Verhältnis zur Herkunftskultur der Großeltern wird dahingehend privatisiert, so dass sie

3 Der Frage nach den Auswirkungen der Konzentration auf die Integration, soll hier nicht weiter nachgegangen werden (vgl. hierzu Alpheis 1990 und Esser 1986).

4 Empirische Belege für die Gültigkeit der ökologischen Phasenmodelle finden sich bei Warner und Srole 1945 sowie Price 1964. 
als Teil der Familiengeschichte interpretiert wird. Beziehungen zwischen Angehörigen der dritte Generation und der autochthonen Bevölkerung werden als normal angesehen.

Gegen den „three-generation-assimilation-cycle“ wurden bald empirische Ergebnisse angeführt, die zeigten, dass sich einzelne ethnische Gruppen nicht schrittweise assimilierten, sondern dass nach mehreren Generationen weiterhin hohe Endogamieraten vorlagen oder dass interethnische Heiraten entlang konfessioneller Trennlinien erfolgten (vgl. Esser 1980 S. 41-42). Prozesse der Segmentation und Re-Ethnisierung werden zum Teil auch unter den heute in Deutschland lebenden Türken beschrieben (Heitmeyer/Müller/Schröder 1997, Straßburger 2001).

\subsubsection{Race-Relation-Cycle}

Das wohl bekannteste und für die weitere Forschung einflussreichste Phasenmodell ist der fünfstufige race-relation-cycle von Robert E. Park und Ernest W. Burgess, dem wird bescheinigt, sie besäßen bereits einige Charakteristiken formaler Theorien, waren aber ähnlicher Kritik wie die Generationsmodelle ausgesetzt (vgl. Price 1969 und Han 2000). Ausgangspunkt des Modells sind die durch Wanderung entstandenen Beziehungen zwischen ethnischen Gruppen, die sich ein Siedlungsgebiet teilen. In der ersten Kontaktphase sind die Beziehungen in der Regel friedlich und dienen dem Kennenlernen. Daraufhin folgt eine Phase, in der die Gruppen in einen Wettbewerb um knappe Ressourcen treten. Aus diesem Wettbewerb, der z.B. auf dem Arbeits- und Wohnungsmarkt stattfindet, ergeben sich Konflikte, die in der dritten Phase Diskriminierung und gewalttätige Auseinandersetzung nach sich ziehen. Im Anschluss daran soll es zu einem Arrangement zwischen den ethnischen Gruppen kommen, das durch Separation und Segregation gekennzeichnet ist. Diese von Trennung gekennzeichnete Lebensweise wird allerdings dem Modell zufolge in der letzten Phase durch interethnische Vermischung überwunden und endet mit vollständiger Assimilation und der sich daraus ergebenden Auflösung der Gruppen (vgl. Price 1969, Treibel 2003). 
Zunächst war das Modell so konzipiert, dass die einzelnen Stufen im Kontakt zwischen ethnischen Gruppen immer in der vorgegebenen Reihenfolge zu gelten hatten, dass der Ablauf zwar verlangsamt, aber nicht aufgehalten werden konnte, und dass es keine Umkehrung des Prozesses geben konnte. In Auseinandersetzung mit der Kritik erweiterte Park das Modell später hinsichtlich des Endzustands, um Kastensysteme und die Möglichkeit der permanenten Minderheit, da es im Falle der indischen Gesellschaft und der Juden in Europa dauerhaft zu keiner vollständigen Assimilation gekommen war. Zu dem diskutierte er die Möglichkeit der Regression, im Sinne von Gruppenkonflikten, die sich nach dem Erreichen der Arrangementphase einstellen können (Park 1928). Weiteren Anlass zur Kritik bot das Fortbestehen der Unterschiede zwischen Kanadiern angelsächsischer Herkunft und Frankokanadiern, die beide als Teil der Kerngesellschaft angesehen werden müssten (Price 1969). Nach dem Modell hätte aber eine allmähliche Angleichung einsetzen müssen, die letztendlich die Auflösung der Gruppenunterschiede zur Folge haben sollte.

\subsubsection{Determinanten der Assimilation}

Die Phasenmodelle bleiben unscharf im Hinblick auf die Erklärung von Assimilation, weil die Prozesse nur als starre Abfolge einzelner Schritte beschrieben werden, ohne dass nach den Gründen der Entwicklung gefragt wird oder Störgrößen berücksichtigt werden. Neben den Phasenmodellen gibt es aber auch einige Arbeiten, die den Prozess der Eingliederung über einzelne Faktoren, wie z.B. ökonomischen Erfolg, soziale Kontakte zu Angehörigen der Aufnahmegesellschaft oder Übernahme der Aufnahmekultur zu erklären suchen.

Ronald Taft unterscheidet in seinem Modell zunächst dazu drei Formen, die monistische, interaktionistische und pluralistische Assimilation von Immigranten. Die monistische Assimilation beschreibt die vollständige Aufgabe der nationalen und kulturellen Identität und das Aufgehen des Individuums in der Aufnahmegesellschaft. Unter interaktionistischer Assimilation versteht Taft, die Entwicklung einer gemeinsamen Identität durch Immigranten und Mehrheitsgesellschaft, bei der Elemente unterschiedlicher Kulturen miteinander kombiniert werden. Mit der Bezeich- 
nung pluralistischer Assimilation ist keine Annäherung verbunden, sondern es verbirgt sich dahinter die gesellschaftliche Akzeptanz kultureller Pluralisierung.

Angelehnt an die monistische Assimilation entwickelte Taft eine Klassifikation des Eingliederungsprozesses auf individueller Ebene, in der die zwei grundlegenden Dimensionen Einstellungen und Verhalten des Wanderers im Angleichungsprozess an eine neue Gruppe unterschieden werden. Auf dieser Grundannahme beruhen sieben Stadien, die ein Immigrant durchlaufen kann, bis er vollständig assimiliert ist, wobei das Erreichen eines Stadiums nicht automatisch die nächste Stufe nach sich zieht, sondern nur die Wahrscheinlichkeit für die nächste Assimilationsstufe erhöht. Daraus ergibt sich, dass in Tafts Modell, im Gegensatz zu den vorgestellten Phasenmodellen, eine teilweise und ungleichmäßige Eingliederung durchaus denkbar ist. Assimilation ist aus dieser Perspektive nicht mehr ein zwangsläufiger Prozess, es besteht aber eine gewisse Wahrscheinlichkeit dafür. Taft formuliert darauf aufbauend eine optimale Sequenz, die den Idealfall der monistischen Eingliederung ohne zusätzliche Hindernisse und Misserfolge beschreibt. Es handelt sich dabei um sieben Facetten der Eingliederung eines Wanderers, die sich auf das kulturelle Wissen, die soziale Interaktion, die Übernahme von Rollenmustern, die soziale und emotionale Identifikation sowie die Konvergenz der Normen beziehen. Diese sieben Facetten werden von Taft hinsichtlich der Ansicht des Immigranten, den jeweiligen Zustand zu erreichen und welches Verhalten er zeigt, um den Zustand zu erreichen, weiter untergliedert. Darüber hinaus wird unterschieden, inwieweit die beabsichtigte Eingliederung durch den Immigranten subjektiv und objektiv erreicht wird (vgl. Taft 1957, 1973). Insgesamt ist das Modell als Wechselspiel von Kognitionen und Handlung des Einwanderers auf der einen Seite und Reaktion der Aufnahmegesellschaft auf der anderen konzeptualisiert.

Dem von Taft entworfenen Modell bescheinigt Esser, dass es über „alle relevanten Elemente einer allgemeinen Theorie der Eingliederung“ (Esser 1980, S.56) verfügt, dass es aber leider über eine Klassifikation der Prozesse nicht hinausgeht.

Ausgehend von seinen Untersuchungen zu den Ursachen und Auswirkungen der Einwanderung nach Israel entwickelte Shmuel N. Eisenstadt 1954 seinen Ansatz zur „Absorption“ der Einwanderer durch die Aufnahmegesellschaft. Voraussetzung 
für die Absorption sind auf Seiten der Einwanderer deren Erwartungen, Hoffnungen und Einstellungen, die auf eine Annäherung gerichtet sein müssen. Ein günstiges Klima für die Absorption in der Aufnahmegesellschaft wird durch die Bereitschaft zur Veränderung, soziale Durchlässigkeit, Aufnahmebereitschaft und über ausreichend Aufnahmefähigkeit gewährleistet. Sofern die Eliten der Immigranten in den schrittweisen Angleichungsprozess eintreten, stellen sie einen zusätzlichen unterstützenden Faktor dar, der über Identifikationssymbolik und Handlungsmodelle die weitere Annäherung der Gruppen fördert. Assimilative Handlungen und Orientierungen gewinnen durch diese Form der Unterstützung innerhalb der ethnischen Gruppen an Gewicht.

Eisenstadt unterscheidet im Rahmen der Eingliederung drei Indikatoren, die für eine gelungene Absorption sprechen. Es ist zunächst die Akkulturation der Immigranten, die vom Erlernen der wichtigsten Alltagstechniken und der Übernahme von Normen und Gewohnheiten gekennzeichnet ist. Infolge dieser Anpassung sollte eine Änderung des Selbstbildes und die Auflösung von Eingewöhnungsproblemen miteinander einhergehen, die als personale Auswirkung der Akkulturation zu sehen sind. Als weiteres Indiz für Absorption der Immigranten nennt Eisenstadt die Dispersion der Immigranten innerhalb der Gesellschaft. Bestehen ethnische Segregation oder ökonomische Diskriminierung fort, so ist dies als Indiz für eine nicht vollständige Absorption der Immigranten zu werten.

Für Eisenstadt ist diese Absorption der Immigranten kein einseitiger Prozess, der nur die Immigranten betrifft. Mit einer steigenden Zahl von eingegliederten Personen werden auch in stärkerem Maße Veränderungen in der aufnehmenden Gesellschaft erwartet (vgl. Eisenstadt 1954).

Für Milton M. Gordon, der sein Modell der Assimilation vor dem Hintergrund der usamerikanischen Gesellschaft entwickelte, sind gesellschaftliche Differenzierungen Ausgangspunkt der Analyse. Aus den bestehenden und untereinander verschränkten ethnischen und vertikalen Ungleichheiten entstehen, unterstützt durch regionale Disparitäten, ethnische Klassenzugehörigkeiten, so genannte „ethclass“ (Gordon 1964). 
Assimilation von Immigranten geschieht demzufolge in einer ethnisch geschichteten Gesellschaft über die Annäherung an eine dominante Kernkultur. Auch Gordon entwirft ein Stufenmodell der Assimilation. Dem idealtypischen Verlauf gemäß wandeln sich zunächst die kulturellen Einstellungs- und Handlungsmuster, daran anschließend dringen die Immigranten in institutionelle Bereiche der Gesellschaft vor und können dann bestehende Heiratsschranken überwinden. Letztendlich entwickeln die Immigranten eine emotionale Verbindung zur Aufnahmegesellschaft. Diese Entwicklung hat zur Folge, dass sich ablehnende Einstellungen und diskriminierende Verhaltensweisen gegenüber den mehr und mehr eingegliederten Gruppen reduzieren und am Ende Interessenkonflikte zwischen den ethnischen Gruppen ihre Basis verlieren. Nach Gordon ist die Akkulturation der erste Schritt zur Assimilation, der auch dann stattfindet, wenn alle weiteren Assimilationsschritte unterbleiben. In der strukturellen Assimilation, die gleichzeitig oder nach der Akkulturation erfolgt, sieht er hingegen die Voraussetzung für alle weiteren Stufen der Assimilation (vgl. Gordon 1964).

Die drei dargestellten Konzepte versuchen alle den Prozess der Anpassung und Integration über eine allmähliche Annäherung der Immigranten an eine Aufnahmegesellschaft zu beschreiben, wobei durchgehend die Perspektive auf eine sich tendenziell auflösende Unterschichtung gerichtet ist. Dabei wird vernachlässigt, dass im Falle einer nicht homogenen Aufnahmegesellschaft die Kernkultur nicht ausschließlich als Bezugspunkt der Assimilation dienen muss. Auch wurde das Ausbleiben von Assimilation und damit das Voranschreiten kultureller Pluralisierung zum Anlass genommen, Assimilation als Beschreibung von Integrationsprozessen völlig abzulehnen (vgl. Glazer/Moyhian 1963). Ein weiterer Kritikpunkt an den Assimilationsmodellen ist, dass jeder assimilative Schritt als unwiderruflich aufgefasst wird, obwohl Forschungsergebnisse auf die Existenz regressiver Tendenzen verweisen (vgl. Herberg 1960, Heitmeyer/Müller/Schröder 1997).

Ganz ähnlich wie bei den Phasenmodellen wird in den Erklärungsansätzen von Taft, Eisenstadt und Gordon der Assimilationsprozess in Form eines Stufenmodells dargestellt. Gegenüber den Phasenmodellen zeichnen sich die Ansätze dadurch 
aus, dass die Vorstellung einer unausweichlichen Assimilation mit feststehender Reihenfolge der einzelnen Schritte fallen gelassen wurde. Die vollständige Assimilation einer Gruppe von Immigranten wird zu einer Möglichkeit unter vielen, für die, abhängig von den Voraussetzungen, lediglich eine mehr oder weniger hohe Wahrscheinlichkeit besteht.

Historisch betrachtet bleibt noch anzumerken, dass die unterschiedlichen Assimilationsmodelle auch dazu benutzt wurden, um politische Programme, wie die nach dem Ersten Weltkrieg in den USA propagierte Americanization im Sinne der angloconformity, zu legitimieren. In der Folge wurde der deskriptive Assimilationsbegriff immer wieder mit der normativen Forderung nach Aufgabe der nationalen und kulturellen Identität gleichgesetzt (vgl. Lucassen 2004). Diese Entwicklung führte zur kritischen Auseinandersetzung mit den theoretischen Ansätzen der Assimilationsforschung, die neue Perspektiven und Schwerpunktsetzungen in die Erforschung der Anpassungsstrategien und Integrationsverläufe von Immigranten anregte.

Im Rahmen der Beobachtung transnationaler Migrationssysteme wird den Assimilationsmodellen entgegengehalten, dass sie für die Erklärung gegenwärtiger Migration nicht weiter angemessen seien, weil die Fokussierung auf die Aufnahmegesellschaft der Wahrnehmung bedeutsamer grenzüberschreitender Bezüge im Wege stehe (vgl. Glick-Schiller/Basch/Blanc-Szanton 1999).

Unter dem Titel „The Return of assimilation?“ beschreibt Rogers Brubaker, anhand der Entwicklung in Frankreich, Deutschland und den USA, wie sich das Verständnis des Assimilationsgedankens in den letzten Jahren zu wandeln beginnt. In seinem zusammenfassenden Überblick zu den Charakteristiken neuerer Assimilationskonzepte konstatiert er eine Verschiebung des Fokus von vollständigem Aufgehen der Individuen hin zum Prozess des Ähnlichwerdens auf der Aggregatebene, der sich von den frühen Phasenmodellen über die an Determinanten orientierten Ansätze bis hin zur Gegenwart fortgesetzt hat. Gleichzeitig mit dieser Verschiebung ist die Bedeutung der kulturellen gegenüber den sozio-ökonomischen Unterschieden immer weiter in den Hintergrund getreten. Damit einhergehend werden sowohl die Immigranten als auch die Aufnahmegesellschaft weniger als homogene, sondern sehr vielmehr als heterogene Gemeinschaften wahrgenommen, zwischen de- 
nen sich Assimilation vollziehen kann. Auch würden Immigranten weniger als Objekte einer sich vollziehenden oder mit ihnen vorzunehmenden Assimilation gesehen, dafür eher als Akteure, die Entscheidungen fällen, deren Konsequenz eine nicht immer intendierte Angleichung der Populationen sein kann. Als Letztes der vielleicht wichtigste Unterschied, den Brubaker benennt: Assimilation ist demnach als ein Bündel aus Prozessen in unterschiedlichen Bereichen zu verstehen, die zum Teil völlig distinkt voneinander verlaufen können (vgl. Brubaker 2001).

\subsection{Ausschluss als ein Grundmuster sozialer Integration}

Die Integrationsverläufe von Immigranten und ihren Nachkommen sind nicht nur an der Tendenz sich über Assimilation der Mehrheitsgesellschaft anzugleichen ausgerichtet. Es existieren auch andere Muster, die prägend wirken. Insbesondere immer dann, wenn wahrnehmbare oder zugeschriebene Kriterien zur Legitimierung von ausschließenden Praxen herangezogen werden. Den Immigranten wird es dadurch erschwert, zwischen Anpassungsstrategien frei zu wählen. Dabei ist es nicht wesentlich, ob die Schließung nun über rassistische, ethnische, religiöse oder linguistische Ideologien gerechtfertigt wird, sehr viel wichtiger für dieses Forschungskonzept ist, dass die jeweilige Diskriminierung institutionalisiert wird. So war z.B. die Einwanderung und permanente Niederlassung von Personen asiatischer Herkunft zwischen $1924^{5}$ und $1952^{6}$ in den USA generell verboten, weil „Asiatics“ selbst in kleiner Zahl als „unassimilable“ galten (vgl. Price 1969, 184). Ähnliche Vorbehalte bestanden zeitweilig auch gegenüber Iren, Juden, süd- und osteuropäischen Einwanderern (Hoerder 2002, S.146). In abgeschwächter Form wurden Praktiken rassistischer Benachteiligung, wie sie der schwarzen Bevölkerung gegenüber mehr oder weniger selbstverständlich waren, auf andere Minderheiten ausgeweitet. Aber nicht nur in den Vereinigten Staaten waren Gesetze und Alltagshandeln von rassistischen Einstellungen geprägt, auch in anderen Einwanderungsnationen, wie Australien, Neuseeland und Kanada fanden ähnliche Regelungen über Jahrzehnte hinweg Anwendung. Jedoch ist die rechtliche Fixierung ausschließender Praxen seit dem Ende des Zweiten Weltkrieges und im Zuge der Entkolonialisierung weitge-

5 Oriental Exclusion Act (für Chinesen bereits 1943 aufgehoben),

6 Mc Carran - Walter Immigration and Naturalization Act 
hend diskreditiert und wurde in den seither vergangenen Jahrzehnten zurückgedrängt. ${ }^{7}$ Allerdings sind damit ausschließende Praktiken in Gesetzen oder im Alltagshandeln nicht verschwunden und noch weit weniger die Folgen überwunden.

Vor diesem Hintergrund wurden Ansätze entwickelt, die zum Teil in expliziter Abgrenzung zum Assimilationsparadigma auf die Entstehung und Reproduktion der Ausschließung von Immigranten und Minderheiten gerichtet sind. ${ }^{8}$ Diese Ansätze in der Adaptationsforschung gehen auf eine fast parallele Entwicklung in der Arbeitsmarkt- und Armutsforschung in Europa und den Vereinigten Staaten zurück. In Europa insbesondere in Frankreich ist sie mit den Begriffen Exklusion und Inklusion verbunden, während sie in den USA eher unter dem Label „underclass“ zusammengefasst wird (Kronauer 2002). Obwohl die Auseinandersetzung mit länderspezifischen Problemlagen diese Ansätze hervorgebracht hat, bestehen grundsätzliche Gemeinsamkeiten. Kennzeichnend für Ausschluss ist dem gemäß zunächst eine marginale Position auf dem Arbeitsmarkt, die sich über Langzeitarbeitslosigkeit, Unterbeschäftigung oder ein unzureichendes Einkommen manifestiert. Der Einkommensverlust schränkt die gesellschaftlichen Teilhabemöglichkeiten ein, so dass allmählich das Konsumverhalten, aber auch die Wohnumstände und kulturelle Aktivitäten den Gegebenheiten immer weiter angepasst werden müssen (vgl. Bös 1995). Charakteristisch ist die sukzessive Reduzierung der sozialen Kontakte auf das Milieu der Benachteiligten (ghetto poor). Zu dem erhöhen sich durch die prekäreren Lebensumstände auch die psychischen Belastungen der Betroffenen, die als zusätzliche Risikofaktoren für den Bestand des familiären Umfelds und der übrigen sozialen Nahbeziehungen angesehen werden müssen. Letztendlich mündet diese Abwärtsspirale aus Einkommensverlust, Einschränkung von Partizipationsmöglichkeiten und Erosion sozialer Netzwerke nicht selten in gesellschaftlicher Isolation (vgl. Castel 1996, Paugam 1994). Die Exkludierten oder Mitglieder der underclass werden zur „Residualkategorie“ der Gesellschaft, der bedingt durch geringe Bildung, mangelnde Qualifikation und Verlust der Arbeitsmentalität ohne Intervention

7 So wurden beispielsweise in der Bundesrepublik in das im Kern auf rassistischer und ethnischer Schließung beruhende Einbürgerungsrecht 1993 und 2000 Elemente des jus-soli eingefügt (vgl. Dornis 2001).

8 Die Kritik richtet sich vornehmlich gegen die normativen Komponenten des Assimilationsansatzes (vgl. 2.1.1). 
von außen nahezu alle Aufstiegsmöglichkeiten versperrt sind (vgl. Kronauer 2002, 72).

Dieser Ansatz aus der Armutsforschung wurde dort zur Analyse der Eingliederung aufgegriffen, wo in der zweiten Hälfte des 20. Jahrhunderts weite Teile der Bevölkerung in Industriegesellschaften von verfestigter Armut betroffen waren. Bedingt durch diese Entwicklung erfuhr insbesondere der „underclass“ Begriff in Nordamerika eine Verengung zur „Black Underclass“. Das Problem von sich verstetigender Armut und Arbeitslosigkeit wurde nun verstärkt im Kontext von Klasse und Rasse diskutiert (z.B. Glasgow 1980, Hall 1980, Wilson 1980, Gilroy 1987, Fainstein 1987, Massey/Denton 1993). Tenor der Arbeiten ist, dass über Segregation innerstädtische Armutsviertel entstanden, in denen die überwiegend schwarze Bevölkerung bedingt durch die geringe Einbindung in den Arbeitsmarkt und die unzureichende staatliche Unterstützung nur geringe Teilhabemöglichkeiten besitzt. Gleichzeitig führen die mit der zunehmenden Verelendung sinkenden Bildungschancen zur Reduzierung der Fähigkeiten, Teilhaberechte einzufordern und dort wo sie bestehen, in Anspruch zunehmen. Dabei wird von einem Teil der Autoren in der weiterhin bestehenden rassistischen Diskriminierung der wichtigste Erklärungsfaktor für diese Entwicklung gesehen (vgl. Fainstein 1987, Massey/Denton 1993).

Dem Verlust von Möglichkeiten und Fähigkeiten zur Teilhabe an zivilen, politischen oder sozialen Prozessen in einer Gesellschaft wird das Konzept der Inkorporation entgegengestellt, das als politische Forderung formuliert, die Verantwortlichkeit für die möglichst gleichberechtigte Einbeziehung von Desintegrierten in alle Bereiche der Gesellschaft bei den Besserintegrierten ansiedelt (vgl. Castles 1987, Anthias/Yuval-Davis 1992). Das Konzept der Inkorporation bzw. die Forderung nach aktiver Inklusionspolitik geht auf Thomas H. Marshalls Analysen zur Teilhabe in entwickelten kapitalistischen Gesellschaften zurück (Marshall 1950). Vor dem Hintergrund der Ausweitung der Bürgerrechte auf die arbeitenden Klassen in Großbritannien stellte er die Analyse der Beziehungen von „social class“ und „citizenship“ ins Zentrum seiner Arbeit. Während die Klassenstruktur in seinen Augen der Ursprung grundlegender Ungleichheiten ist, stehen die Bürgerrechte für das entgegengesetzte Bestreben, den Mitgliedern einer nationalen Gemeinschaft gleichberechtigte Teilhabe zu ermöglichen. Marshall unterscheidet dazu drei Elemente des 
Bürgerrechts: „I shall call these parts, or elements, civil, political and social“ (Marshall 1950, 10). Wobei mit bürgerlichen Rechten klassische Eigentums- und Freiheitsrechte bezeichnet werden und unter politischen Rechten vor allem das aktive und passive Wahlrecht verstanden wird. In Bezug auf diese beiden Elemente stellt Marshall allerdings fest, dass sie nur wenig zur Veränderung der Ungleichheiten zwischen den Klassen, aber wesentlich zur Integration des Staates beigetragen haben (ebd., 24-26). Erst die Etablierung sozialer Rechtsansprüche, auf ein Minimum an sozialer Sicherheit und wirtschaftlichem Wohlstand, ist seiner Auffassung nach dafür ausschlaggebend gewesen, dass die Klassenunterschiede in stärkerem Maß abgeschwächt werden konnten.

In Abhängigkeit von den spezifischen Verhältnissen der jeweiligen Gesellschaft werden in der Diskussion des Ausschlusses einzelne Elemente oder auch Kombinationen der Bürgerrechte im Sinne Marshalls eingefordert. In den USA lässt sich die Debatte durch Begriffe wie staatliche Wohlfahrt und positive Diskriminierung kennzeichnen (Loury 2000). Während in Bezug auf die Situation der Ausländer in Deutschland lange Zeit die rechtliche Gleichstellung durch ein erleichtertes Einbürgerungsverfahren im Vordergrund stand, hat sich die Diskussion über theoretische Gräben hinweg seit der Änderung des Einbürgerungsrechts und mit den PISA-Ergebnissen in noch verstärkter Form in Richtung des Ausgleichs struktureller Nachteile insbesondere im Hinblick auf Bildungs- und Erwerbsbeteiligung verschoben (Kirsten 2000, Radtke 2004, Hunger/Thränhardt 2004). Im Falle von Immigranten ohne geregelten Aufenthaltsstatus (in der Illegalität lebend) beziehen sich die Forderungen auf alle Ebenen der von Marshall charakterisierten Bürgerrechte (vgl. Haines/Rosenblum 1999, Rosner 2001, Bade 2002, Stobbe 2004).

Mit der Verlagerung der Forschungsperspektive von der Annäherung hin zur Existenz und Reproduktion von Ausschluss wurde versucht, ein normatives Gegenkonzept zum Assimilationsansatz zu entwickeln. Allerdings ist dies nur dort gelungen, wo man sich gegenüber den normativen Interpretationen der Assimilation abgrenzen konnte. Gemessen an den konkreten Forschungsinhalten bestehen zwischen beiden Ansätzen große Gemeinsamkeiten. Sieht man von den normativen Prämissen $a b$, so thematisieren beide Forschungsrichtungen die Einbeziehung von Immigranten bzw. Minderheiten in alle Bereiche einer Gesellschaft. Bei allen Ähnlich- 
keiten bestehen aber auch wesentliche Unterschiede. Während im Assimilationsansatz die Immigranten als Akteure gesehen werden, deren Verhalten die weiteren Abläufe maßgeblich bestimmen, sind es gemäß der Prämissen des Ausschlusskonzepts die herrschenden Gruppierungen, in aller Regel die autochthone Bevölkerung, die über die Positionierung der Gruppen in der Gesellschaft entscheidet. Die Erklärung der Entstehung und Reproduktion sozialer Ungleichheiten zwischen Immigranten bzw. Minderheiten und der Mehrheitsgesellschaft zielt dementsprechend letztlich auf eine mehr oder weniger deutlich ausgeprägte Schuldzuweisung. Auf dieser normativen Setzung aufbauend werden die gesellschaftlichen Entwicklungen bewertet und integrationspolitische Maßnahmen begründet.

\subsection{Kulturelle Pluralisierung und transnationale Bezüge}

Neben den Forschungslinien, die auf die Entwicklung der Annäherung von autochthoner und allochthoner Bevölkerung bzw. deren Verhinderung gerichtet sind, gibt es Ansätze, in deren Rahmen die Erforschung der Anpassungsstrategien von der Konstanz in den Unterschieden her entwickelt wird. Mit dieser Perspektive ist sowohl das Konzept der kulturellen Pluralisierung, als auch der Transnationalismus verbunden.

\subsubsection{Kulturelle Pluralisierung von Einwanderungsgesellschaften}

Ein klassisches Verhaltensmuster von Immigranten in einer Aufnahmegesellschaft ist die weitgehende Bewahrung der ethnischen oder kulturellen Identität, die es ermöglicht, die Nutzbarkeit des mitgebrachten sozialen und spezifisch-kulturellen Kapitals zu erhalten (vgl. Esser 1997). Diese Strategie der Anpassung setzt auf Dauer die Bildung ethnischer oder kultureller Gemeinschaften voraus, ohne deren Unterstützung die Produktion spezifischer Güter und die Pflege identitätsstiftender Traditionen erheblich erschwert sind. Die weitere Verwendbarkeit des kulturellen Kapitals ist sogar zwingend an die Existenz der entsprechenden Gruppe gebunden. Gleich- 
zeitig erleichtert die Einbindung in eine ethnisch geprägte Kolonie zunächst die Integration von Immigranten in die Aufnahmegesellschaft (vgl. Elwert 1984). Für die aufnehmende Gesellschaft können ethnische Kolonien von Vorteil sein, weil erforderliche Integrationsleistungen durch die Einwanderercommunities erbracht werden, ohne dass Ressourcen der autochthonen Mehrheitsgesellschaft für diesen Prozess bereitgestellt werden müssen.

Die räumliche Konzentration von Immigranten mit ähnlicher ethnischer Herkunft ist in Einwanderungsgesellschaften immer wieder zu beobachten. Beispiele für diese Anpassungsstrategie lassen sich in der Migrationsgeschichte viele finden, ob es z.B. türkische Einwanderer in westdeutschen Großstädten, chinesische in Australien oder lateinamerikanische Gruppen in den USA sind. Welche Entwicklung eine solche ethnische Kolonie nimmt, ist dabei nicht von vornherein klar. Einige, wie z.B. die im Ruhrgebiet verbliebenen Gruppe der Polen, assimilieren sich vollständig und sind nach wenigen Jahrzehnten nahezu verschwunden, andere, wie die Siebenbürger Sachsen in Rumänien, bleiben über Jahrhunderte im Kern bestehen.

Die kulturelle Pluralisierung von Gesellschaften ist immer ein wesentlicher Aspekt der Integrationsforschung gewesen, allerdings lag der Schwerpunkt lange Zeit auf der normativ gefassten Assimilation. Kultureller Pluralismus wurde demgemäß im Sinne einer unvollständigen und unzureichenden Assimilation verstanden, die es abzuschließen galt. Erst in der zweiten Hälfte des 20. Jahrhunderts wurde über die sozialwissenschaftliche Rezeption sozialer Bewegungen eine neue Perspektive im Hinblick auf kulturelle Pluralisierung entwickelt. Neben dem Kampf gegen die Rassentrennung in den USA war es vor allem die politische Entwicklung in Kanada, die auf eine kulturelle Gleichstellung der frankophonen mit den englischsprachigen Kanadiern abzielte und damit den Grundstein für das normative Integrationskonzept des Multikulturalismus legte. ${ }^{9}$ Unter dem Label ethnic revival wurde neben vielfältigen kulturellen Initiativen auch eine große Anzahl Forschungsarbeiten veröffentlicht. Die Rückbesinnung auf die eigene Herkunftskultur wurde mit der Forderung

9 The Royal Commission on Bilingualism and Biculturalism empfahl 1970 die gleichberechtigte Anerkennung der bestehenden kulturellen Unterschiede in Kanada. 1971 wurde die Politik des sogenannten Multikulturalismus zur politischen Leitidee der kanadischen Minderheitenpolitik. (vgl. Salojee 2003, S. 10) 
nach Akzeptanz unterschiedlicher Ethnizität verbunden, die in Abgrenzung zum Bild des amerikanischen Schmelztiegels, als salad bowl beschrieben wird.

In Deutschland wurde die Anerkennung kultureller Pluralisierung zu einem politischen Thema, nachdem unübersehbar geworden war, dass trotz der in den achtziger Jahren zur Rückkehrförderung bereitgestellten Mittel, große Teile der ehemaligen Gastarbeiterfamilien es vorzogen, in Deutschland zu bleiben. Ende der Achtziger und Anfang der Neunziger wurde das Modell zunächst von einigen wissenschaftlichen und politischen Autoren unter dem Begriff der multikulturellen Gesellschaft aufgegriffen und als auf Deutschland übertragbar angeführt (z.B. Ruhloff 1989, Leggewie 1990; Baringhorst 1991; Cohn-Bendit/Schmid 1992). Daraufhin entstand eine Diskussion, die den Begriff multikulturelle Gesellschaft zu einem Reizwort werden ließ (vgl. Mintzel 1997, S.24-38), dem der Leitkulturbegriff entgegen gesetzt wurde. Als politisches Konzept war die Anerkennung kultureller Pluralisierung in Deutschland damit vorläufig gescheitert, obwohl empirisch betrachtet durch neue Immigrationsschübe die Pluralisierung weiter vorangeschritten ist.

International betrachtet, wurde das Konzept theoretisch weiter ausgearbeitet. Charles Taylor und Will Kymlicka, die zurzeit prominentesten Theoretiker des Multikulturalismus, versuchten gleichermaßen im Rahmen ihrer Sozialtheorien eine Legitimationsgrundlage für die Gleichberechtigung ethnischer und kultureller Kollektive innerhalb eines Nationalstaates zu liefern. Auf der Basis der Anerkennung ethnischer und kultureller Unterschiede und vermittelt über die jeweilige ethnische Gemeinschaft soll dementsprechend die Integration der Individuen in die Gesellschaft erfolgen (vgl. Taylor, 1992). Kennzeichnend für Kollektive sind dabei Sinngrenzen oder Realitätskonzepte, die als implizites Hintergrundwissen der Akteure beschrieben werden. ${ }^{10}$ Wird im Verhältnis zur Umwelt dann eine Differenz zu den Sinngrenzen einer anderen Gemeinschaft wahrgenommen, entwickelt sich daraus die ethnische Identität. Kymlicka ergänzt in seiner idealtypischen Definition noch den Aspekt der räumlichen Abgrenzbarkeit kultureller Gemeinschaften. Multikulturalismus entsteht dann, wenn innerhalb eines Nationalstaates mehrere ethnische oder kulturelle Kollektive nebeneinander existieren, die gleichermaßen Anspruch auf Aner-

10 Hier besteht große Ähnlichkeit zu dem, was Esser als spezifisches oder kulturelles Kapital bezeichnet (vgl. Esser 1997). 
kennung ihrer Kultur haben. ${ }^{11}$ Unterstellt wird dabei, dass die Gemeinschaften sich als different nach außen wahrnehmen und nach innen homogen erscheinen. Die einzelnen Individuen werden als Träger der gemeinsamen Kultur verstanden und in ihrer Summe bilden sie dann die kulturelle Gemeinschaft (vgl. Reckwitz, 2001 S.181-185).

Neben dieser gibt es noch eine weitere Perspektive zur kulturellen Pluralisierung von Gesellschaften, die den Kulturbegriff angelehnt an die Arbeiten Wittgensteins definiert (ebd.). Dabei wird die Vorstellung der Homogenität einer Kultur aufgegeben und die Verbindung aus Kultur, Gemeinschaft und Individuen eher als Ausnahmefall betrachtet. Ausgehend von einer Definition der Kultur, als Sammlung geteilter sozialer Praktiken und Wissensordnungen, erfolgt eine Entkopplung der Kultur von den Akteuren. Der Einzelne kann Träger unterschiedlicher sozialer Praktiken und Wissensordnungen sein, mit deren Hilfe die Teilhabe an verschiedenen Kulturen erfolgt. Aus dieser Idee kultureller Hybridität heraus ließen sich im Rahmen des Multikulturalismus neue Fragestellungen entwickeln. Fragestellungen, die stärker auf die Identitätsentwicklung und die Nutzung unterschiedlicher kultureller Ressourcen abzielen, was eine Öffnung des Multikulturalismusansatzes in Richtung des Transnationalismus ermöglicht (vgl. Reckwitz 2001).

Die Konzepte des Multikulturalismus sind sowohl in der Öffentlichkeit, als auch in der Wissenschaft über Jahre hinweg kontrovers diskutiert worden, aber vor allem die sozialen und politischen Entwicklungen in einigen Einwanderungsnationen, die eine multikulturelle Integrationspolitik verfolgten, hatten einen relativierenden Einfluss auf die jüngsten Debatten (vgl. Bade/Bommes 2004). Nicht die Politik der Anerkennung wird dabei in Frage gestellt, es sind vielmehr die besorgniserregenden aber nicht intendierten Folgen, die den politischen und theoretischen Grabenkämpfen ein Ende bereitet haben. So entstanden trotz der Anerkennung ethnischer Gemeinschaften gesellschaftliche Segmentationen, die in der Mehrzahl der Fälle sukzessive auf eine dauerhafte Unterschichtung der Mehrheitsgesellschaft hinauslau-

11 Aber selbst im erklärtermaßen multikulturellen Kanada wird eine Hierarchie im Hinblick auf die Identität erwartet. Der jeweiligen ethnischen Identität soll eine einheitliche kanadische übergeordnet sein, die mit unity-within-diversity umschrieben wird. Teil dieser erwarteten Einheit ist die Anerkennung gemeinsamer Grundwerte, die letztendlich im Konfliktfall Vorrang vor denen der ethnischen Gemeinschaft haben (Geissler 2003). 
fen (Böcker/Thränhardt 2003, Baringhorst 2003, Castles 1998, Ring 1998). ${ }^{12}$ Deutlich wird an diesen Entwicklungen, dass sich aus den politischen Zielsetzungen der Gleichberechtigung von ethnischen Gruppen nicht zwangsläufig eine sozial-strukturelle Gleichstellung ergeben muss. Beate Winkler verweist in diesem Zusammenhang darauf, dass eine kulturell-pluralisierte Gesellschaft nur dann Chancengleichheit ermöglichen kann, wenn Anerkennung auch mit sozialem Ausgleich verbunden ist (Winkler 1993).

\subsubsection{Transnationale Bezüge der kulturellen Pluralisierung}

Mit der Bewahrung ethnischer bzw. kultureller Identität ist noch eine weitere Anpassungsstrategie verbunden, die für Einwanderungsgesellschaften typisch zu sein scheint. Immigranten tendieren nicht nur dazu ethnische Kolonien zu bilden, sie unterhalten auch oft enge Kontakte zu ihrer Herkunftsgesellschaft; seltener zu Kolonien der eigenen Ethnie in anderen Nationalstaaten. ${ }^{13}$ Unterstützt durch technische Entwicklungen im Bereich der Telekommunikation und durch die erhöhte Mobilität fällt der Aufbau und die Pflege derartiger Beziehungsgeflechte heute leichter. Sofern diese Beziehungen über nationalstaatliche Grenzen hinweggehen, wird von der Bildung transnationaler Systeme oder Sozialräume gesprochen (GlickSchiller/Basch /Blanc-Szanton 1999, Vertovec 1999, Faist 2000, Pries 2001). In Abgrenzung zum ökonomisch geprägten Begriff der Globalisierung hat sich der Terminus Transnationalismus für die über nationalstaatliche Grenzen hinweggehenden individuellen Beziehungen etabliert.

Die Anpassungsstrategie eines idealtypischen Transmigranten an seine Lebensumstände besteht aus dem fortwährenden Wechsel zwischen unterschiedlichen Nationalgesellschaften, wobei dieser nicht durch die nationalen, sondern durch die sozialen Bezüge determiniert wird. Die Strukturierungskraft nationaler Staaten für soziale Beziehungsgeflechte verliert dem Ansatz zufolge an Bedeutung und es bilden

12 Eine Ausnahme stellen chinesische Einwanderer in Nordamerika dar. In Kanada und den USA sind Zeichen einer Überschichtung der Mehrheit durch chinesische Einwanderer festzustellen. (vgl. Geißler, 2003)

13 So z.B. die kurdischen Flüchtlinge in Westeuropa, die untereinander und mit der (transnationalen) „Heimat“ engen Kontakt halten. (vgl. Wahlbeck, 1998) 
sich zunehmend pluri-lokale transnationale Sozialräume, die sich auf die Identifikation und Sozialstruktur auswirken. Mit der schwindenden Bindung des Individuums an eine nationale Gesellschaft wird erwartet, dass transnationale Bezüge die Entwicklung von Mehrfach- und hybriden Identitäten fördern (Pries 2002).

Im Rahmen des Transnationalismusansatzes werden soziale, kulturelle, politische und ökonomische Beziehungen betrachtet, wie z.B. die Heiratsmuster türkischer Immigranten in Deutschland, die transnationalen Geschäftstätigkeiten türkischer Gewerbetreibender oder die Pendelmigration mexikanischer Personen und Familienverbände zwischen den USA und Mexiko (Straßburger 2001, Faist 1999, Espinosa/Massey 1999).

Transnationale Bezüge stellen einen wichtigen Pfeiler ethnischer Kolonien dar, weil über den Austausch mit der Herkunftskultur oder einer anderen Kolonie die eigene ethnische Identität stabilisiert und weiterentwickelt werden kann. Gleichzeitig bietet der Kontakt dem Einzelnen die Möglichkeit zusätzliche Ressourcen zu erschließen, die auch für den Aufbau und Erhalt der „Diasporagemeinde“ genutzt werden können, zumal einige Ressourcen in der Aufnahmegesellschaft möglicherweise nicht vorhanden oder nur schwer zugänglich sind. ${ }^{14}$ Nicht zuletzt besteht der Nutzen transnationaler Beziehungen in der Exitoption, denn sollte die Aufnahmegesellschaft nicht den Erwartungen genügen, dann sind nicht alle Brücken abgebrochen oder auch neue Verbindungen geknüpft. Dem entsprechend bezeichnet Ludger Pries die grenzüberschreitenden Aktivitäten als „transnationale Lebens- bzw. häufig Überlebensstrategien“ von Migranten (Pries 2003, 25)

Aber nicht alle Forschungsfelder, die unter dem Label Transnationalismus zusammengefasst werden, sind wirkliches Neuland der sozialwissenschaftlichen Forschung. Die Innovation besteht eher darin, die grenzüberschreitenden Anpassungsmuster der Immigranten in voller Breite zu würdigen und nicht auf die im Aufnahmeland als relevant bewerteten Prozesse wie z.B. Transferzahlungen und Remigration zu reduzieren sowie die Bedeutung von transnationalen Bezügen für die Identitätsentwicklung von Migranten herauszuarbeiten.

14 Ein typisches Beispiel ist die Praxis der Anwerbung von türkischen Imamen durch türkisch-islamische Gemeinden in Deutschland. (Vgl. Zentrum für Türkeistudien, 2005, S. 9) 


\subsection{Zusammenfassung und Zwischenfazit}

Das wissenschaftliche Interesse an Immigranten war zu Beginn der sozialwissenschaftlichen Forschung dominiert von der Frage, welche Prozesse bis zur vollständigen Assimilation der Immigranten ablaufen. In den frühen theoretisch orientierten Arbeiten wurde der Assimilationsprozess demgemäß nach zeitlichen Aspekten klassifiziert. Als das für die spätere Forschung einflussreichste Modell dieser Periode gilt der race-relation-cycle von Park und Burgess. Die Phasen- oder Sequenzmodelle der Assimilation waren auf einen idealtypischen Verlauf der Eingliederung ausgerichtet, der fast ausschließlich an den Interessen der Aufnahmegesellschaft orientiert ist. Deshalb konnten diese starr konzipierten Modelle nur wenig zum Verständnis nicht-idealtypisch verlaufender Integrationen beitragen. In der Auseinandersetzung mit diesen Defiziten wurde der Assimilationsansatz weiterentwickelt, indem die Determinanten der Assimilation stärker in den Mittelpunkt gerückt wurden. Die vorgestellten Modelle von Taft, Eisenstadt und Gordon haben gemeinsam, dass die Assimilation in Subdimensionen untergliedert wird. Für den Prozess der Eingliederung werden übereinstimmend das Erlernen kultureller Grundtechniken, der Aufbau sozialer Kontakte und die Entwicklung einer Identifikation mit der Aufnahmegesellschaft als wesentliche Dimensionen der Assimilation betrachtet. Eisenstadt betont zudem, dass Immigranten innerhalb der Gesellschaft verteilt anzutreffen sein sollten, weil Diskriminierung, Segregation und ökonomische Segmentation als Indiz für eine nicht abgeschlossene Absorption anzusehen sind. Gordons Modell beinhaltet in diesem Sinne weitere Subdimensionen der Assimilation. Dabei werden die Einbindung des Individuums in das Statussystem der Gesellschaft als strukturelle Assimilation, das Fehlen von Vorurteilen und Diskriminierung in der Aufnahmegesellschaft, als einstellungs- und verhaltensmäßige Akzeptanz und das Fehlen von Wert- und Machtkonflikten als zivile Assimilation bezeichnet. Im Gegensatz zu den frühen Klassifikationen mit feststehender Phaseneinteilung und unausweichlicher Assimilation wird in den an Determinanten orientierten Modellen die vollständige Eingliederung zu einer Möglichkeit.

Aber nicht alle Entwicklungen im Rahmen von Immigration können ohne weiteres mit Assimilation erklärt werden. Es lassen sich auch Tendenzen erkennen, die auf die Verhinderung von Annäherung abzielen und den Immigranten bzw. Vertretern 
inferiorer Bevölkerungsgruppen soziale Mobilität weitgehend verwehren. Verbunden mit den Begriffen Exklusion und underclass entwickelte sich daraus ein weiterer Zweig der Integrationsforschung, der das Ausbleiben von Assimilation auf schließende Praxen zurückführt. Aufbauend auf den Analysen von Marshall zur gesellschaftlichen Teilhabe wurden die Wirkungen rechtlicher Benachteiligungen und sozialer Missstände auf die Integration von Immigranten und Minderheiten erforscht. Als Antwort auf das normative Assimilationsverständnis wurde das Konzept der Inkorporation formuliert, mit dem die Möglichkeit zur zivilen, politischen und sozialen Teilhabe unabhängig von Merkmalen, wie z.B. Rasse, Herkunft, Ethnie, Sprache, Religion zu einem gesellschaftlichen Ziel erklärt wird.

Ausgehend von der Neigung zum Erhalt ethnischer bzw. kultureller Identität in Immigranten- und Minderheitencommunities wurde im Anschluss an die Emanzipationsbestrebungen im französisch geprägten Quebec und den ethnic-revival in den USA die kulturelle Pluralisierung von Gesellschaften zu einem weiteren Bereich sozialwissenschaftlicher Auseinandersetzung mit der Integrationsproblematik. Vor dem Hintergrund der Anerkennung ethnischer Differenz wird im Rahmen dieses Ansatzes Assimilation als normatives Integrationskonzept abgelehnt. Auf Basis der Anerkennung kultureller Pluralisierung sind unterschiedliche Integrationskonzepte entwickelt und diskutiert worden, die unter dem Begriff Multikulturalismus zusammengefasst werden. Klassischerweise werden ethnische Gemeinschaften intern als homogen und in Abgrenzung zu anderen Ethnien als different betrachtet. Kennzeichnend für diese Multikulturalismusansätze ist, dass die Individuen als Träger der Kultur in ihrer Summe die Gemeinschaft bilden. Damit werden, bedingt durch die herausgehobene Stellung der ethnischen und kulturellen Gemeinschaft im Multikulturalismus, in erster Linie die ethnischen Kollektive zu den eigentlichen Adressaten einer Integrationspolitik (Bade/Bommes 2004). Aber die Anerkennung kulturell differenter Gemeinschaften schließt nicht per se aus, dass es trotzdem zu Segmentations- und Stratifikationsprozessen kommt, wie am Beispiel einiger klassischer Einwanderungsnationen festgestellt werden konnte.

Mit der Tendenz, die ethnisch-kulturelle Identität zu erhalten, ist auch das Konzept der Transnationalisierung verbunden, das an den grenzüberschreitenden Beziehungen und Aktivitäten der Immigranten ansetzt. Insbesondere die Kontakte zur 
Herkunftsgesellschaft stehen dabei im Vordergrund des sozialwissenschaftlichen Forschungsinteresses. Der Transmigrant als Mittler und Pendler zwischen den Kulturen wird weniger als Mitglied einer homogenen ethnisch-kulturellen Gemeinschaft wahrgenommen, sondern eher als Träger unterschiedlicher kultureller Wissensordnungen und Praktiken. Die Entstehung widersprüchlicher und spannungsgeladener hybrider Identitäten wird auf die wechselnden ethnisch-kulturellen Einflüsse zurückgeführt.

Werden die dargestellten Forschungslinien ohne die jeweiligen normativen Programme bezogen auf die Anpassungsstrategien und die Integrationsverläufe betrachtet, so wird deutlich, dass die einzelnen Ansätze auf Teilaspekte der Strategien und Verläufe des Integrationsgeschehen ausgerichtet sind.

Überspitzt formuliert: Während sich die Befürworter des Assimilationsansatzes dem Ähnlichwerden von Immigranten und Mitgliedern der Mehrheitsgesellschaft widmen und dabei alle anderen Entwicklungen tendenziell als Hemmnisse oder Verzögerungen interpretieren, fokussiert der Inklusions-Exklusionsansatz vornehmlich auf die Barrieren, die von der Aufnahmegesellschaft gegenüber den Immigranten errichtet werden und die Konzepte der kulturellen Pluralisierung beziehen sich in erster Linie auf das spezifisch Ethnische bzw. der Transnationalismus auf die grenzüberschreitenden Aktivitäten und vernachlässigen dabei tendenziell die zwangsläufige Einbettung in den aufnehmenden Kontext, der oft erheblich durch die Kernkultur der Mehrheitsgesellschaft geprägt ist (Brubaker 2001). Aber Assimilation, kulturelle Pluralisierung, Aufbau transnationaler Sozialräume und gesellschaftliche Schließungsprozesse sind nicht alternative Prozesse, sondern dahinter stehen Strategien der beteiligten Akteure und - soweit die Möglichkeit besteht - werden sie auch immer gleichzeitig anzutreffen sein. In welchem Ausmaß sie auftreten und welche Rolle sie hinsichtlich der ökonomischen Verhältnisse, der sozialen Einbindung und der Identitätsentwicklung einer spezifischen Immigrantengruppe erlangen, sind empirisch zu klärende Fragen, die in großem Maße von den jeweiligen Rahmenbedingungen beeinflusst werden. Immigration bewirkt in jedem Fall Veränderung, dem das Handeln der beteiligten Individuen Rechnung trägt. Aus dieser vereinfachenden Perspektive sind Assimilation, Einbürgerungsangebote, Bildung ethnischer Kolonien, Aufbau transnationaler Bezüge, Anerkennung kultureller Unter- 
schiede, Schließungsprozesse und Abwanderung häufig vorzufindende Strategien, mit denen auf die neue bzw. auf die durch Zuwanderung veränderte Umwelt reagiert wird. ${ }^{15}$

Die Integrationsforschung wird der Vielfalt des gleichzeitigen Nebeneinanders von Reaktionsstrategien und komplexen Kombinationsmustern, die auf Basis der unterschiedlichen Gegebenheiten zu sehr spezifischen Integrationsverläufen führen, nur unzureichend gerecht, wenn das Forschungsfeld aus der Perspektive eines Teilbereichs betrachtet wird. Darüber hinaus wird es durch die isolierte Betrachtung schwieriger, Verhaltensmuster und Entwicklungen in ihrer Gesamtheit nachzuvollziehen oder gar zu prognostizieren. Die Kontroversen zwischen Vertretern einzelner Ansätze darüber, welches Forschungsparadigma durch die neueren Entwicklungen als überholt und inadäquat zu gelten habe, gehen am Kern der Problematik vorbei und erscheinen oft politisch motiviert zu sein. Solange die von Hartmut Esser schon 1980 beklagte „chaotische Zersplitterung“ der Forschungsarbeiten vorherrscht und sich mit Verallgemeinerungen spezifischer Forschungsergebnisse verbindet, werden auftretende Widersprüche immer wieder Anlass zu Auseinandersetzungen konkurrierender Schulen bieten (vgl. Esser 1980, 12). Ansätze, die auf spezifische Problemfelder ausgerichtet sind, können sich gegenseitig ergänzend möglicherweise ein Gesamtbild vermitteln, aber es ist m. E. wenig sinnvoll, aus dem verstärkten Auftreten transnationaler Prozesse einen Bedeutungsverlust der Assimilation abzuleiten, noch die Entwicklung kultureller Pluralisierung zu vernachlässigen, weil in einigen klassischen Einwanderungsländern das politische Projekt des Multikulturalismus vermutlich mit zu sozialen Unterschieden zwischen ethnischen Gruppen beigetragen hat. Das Verständnis und die einseitige Betonung transnationaler Systeme wird wenig zur Verbesserung der Lebensverhältnisse von Immigranten beitragen, wenn nicht auch die Strukturen und Prozesse in den jeweiligen Aufnahmegesellschaften untersucht werden. ${ }^{16} \mathrm{Um}$ die Integrationsverhältnisse mit den wesentlichen dahinter liegenden Anpassungsstrategien und Verläufen erfassen zu können, ohne dabei von vornherein schon über die theoretischen Setzungen relevante Prozesse auszuschließen, bedarf es eines ergebnisoffenen Ansatzes. Dieser sollte die Breite

15 Dabei handelt es sich nicht unbedingt um neue Reaktionen, wie an den Ergebnissen der historischen Migrationsforschung zu erkennen ist (z.B. Sievers 1981, Hoerder 1985; Herbert 2001; Bade 2002).

16 Insofern ist Ludger Pries zuzustimmen, wenn er Transnationalisierung als Ergänzung des klassischen Migrationsverständnisses bezeichnet (vgl. Pries 2003, 23). 
eines Phänomens widerspiegeln, das über Generationen hinweg als fragil, jederzeit revidierbar und zum Teil in distinkten Prozessen verlaufend beschrieben wird (vgl. Pries 2003, 33-34; Brubaker 2001, 544). Die vorgestellten Ansätze beziehen sich aber nur auf Teilaspekte der Integrationsverhältnisse, allerdings haben sie eines gemeinsam: Alle Ansätze rekurrieren auf Anpassungsstrategien und die sich daran anschließenden typischen Verlaufsmuster. Selbst die eher philosophischen Reflexionen über den Fremden und seine Funktion in einer Gesellschaft können nicht umhin, auf die Akteure Bezug zu nehmen (Nassehi 1995; Bielefeld 1998; Radtke 1998). Wenn nicht lediglich aus einer beschreibenden Perspektive heraus die Thematik behandelt wurde, dann sind bislang in den meisten Fällen erklärende Modelle mittlerer Reichweite entwickelt worden, ohne diese theoretisch an das Handeln der Individuen anzubinden und die intendierten wie die Fülle der nicht intendierten Folgen dabei im Auge zu behalten. Am Maßstab einer deduktiv-nomologischen oder zumindest induktiv-statistischen Erklärung gemessen, erfüllt ein großer Teil der Forschungsliteratur nicht die Anforderungen, die an eine in diesem Sinne wissenschaftliche Erklärung gestellt werden (vgl. Hempel/Oppenheim 1948 135-175; Opp 1995 50-55; Esser 1999b, 119-140). Ein Ansatz, der die unterschiedlichen Forschungsperspektiven in Form eines Erklärungsmodells der Anpassungsmuster von Immigranten und autochthoner Bevölkerung aufeinander beziehend bündelt, ist bislang nicht vorgelegt worden. Im Hinblick auf die Erklärung des Verhaltens von Immigranten und der Integrationsverläufe sind die neueren Assimilationsmodelle, in der am methodologischen Individualismus ausgerichteten Form, am vielversprechendsten, solange Assimilation als multidimensionaler Prozess definiert wird - der weder vollständig durchlaufen werden muss, noch unumkehrbar ist - und nicht eine Umdeutung in ein politisches Programm erfährt (Brubaker 2001; Lucassen 2004). 


\section{Abwanderung und Einbürgerung: Stand der Forschung}

Die Frage nach den Determinanten von Abwanderung aus und Einbürgerung in eine Aufnahmegesellschaft stehen im Rahmen der wissenschaftlichen Auseinandersetzung mit Immigration und ihren Folgen nicht unbedingt im Fokus des wissenschaftlichen Interesses. Im Vordergrund der Debatten stehen akutere Probleme, wie die Entwicklung ethnischer Stratifikationen, Kontroversen hinsichtlich der für die Gesellschaft allgemeingültigen Prinzipien oder auch Konflikte um die symbolische Aneignung des öffentlichen Raums. Aber Abwanderung und Einbürgerung stellen gewissermaßen Eckpunkte der Integration dar. Einbürgerung kann in diesem Sinne als Ausdruck eines dauerhaften Interesses an der Aufnahmegesellschaft und der Anerkennung dieses gewertet werden und ist damit prototypisch für eine gelungene Eingliederung. Im Gegensatz dazu steht Abwanderung für eine weitgehende Abkehr von der Aufnahmegesellschaft und ist - sofern angestrebt - ein Indiz für missglückte Integration. Die Perspektive Abwanderung und Einbürgerung gleichzeitig im Kontext der Integration zu untersuchen, ist in der Forschungsliteratur nicht üblich, wenn überhaupt, werden beide Vorgänge isoliert voneinander betrachtet.

Im Folgenden sollen jeweils nach einer kurzen Konkretisierung des Begriffs, das Ausmaß und die durch empirische Forschung herausgearbeiteten bestimmenden Faktoren der Abwanderung (3.1) bzw. der Einbürgerung (3.2) dargestellt werden, um dann auf den Ergebnissen aufbauend, Argumente für eine gemeinsame Betrachtung herauszuarbeiten (3.3).

\subsection{Abwanderung: Absichten und Determinanten}

Migration ist ganz allgemein ein Ortswechsel, allerdings bestehen keine einheitlichen Regeln, ab wann eine Person als Immigrant gilt. Während die Vereinten Nationen empfehlen, einen Wohnortswechsel als Migration anzusehen, wenn dieser länger als fünf Jahre anhält, wird in der Bundesrepublik jeder tatsächliche Gemeindewechsel als Migrationsvorgang interpretiert und statistisch erfasst (vgl. Han 2000, 
7). Werden nationalstaatliche Grenzen während des Migrationsvorgangs überschritten, so wird dies im Unterschied zur Binnenmigration als internationale Migration bezeichnet.

\section{Abbildung 3.2.1a: Schema der Emigration-Remigrations-Schleifen mit zwei Auf-} nahmegesellschaften

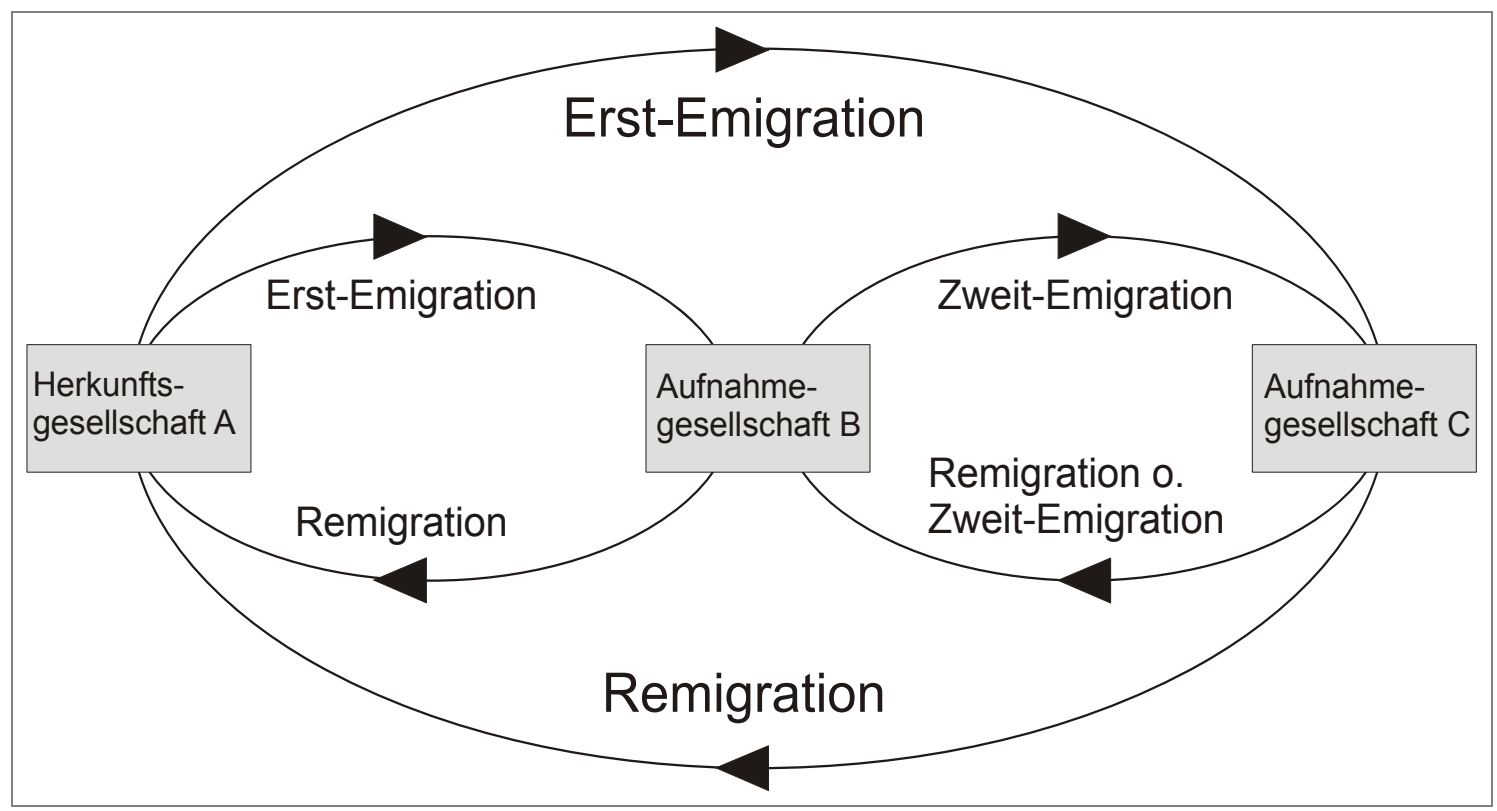

Um die Zuverlässigkeit der Forschungsergebnisse hinsichtlich des Zu- und Abwanderungsverhaltens einschätzen zu können, ist es zudem erforderlich, darüber hinausgehende Unterscheidungen von Wanderungsvorgängen vorzunehmen, denn es gibt eine Reihe unterschiedlicher Ortswechsel, die vereinheitlichend unter Migration zusammengefasst werden (vgl. Abb. 3.2.1a).

Anhand der in Abbildung 3.2.1a skizzierten Wanderungsbewegungen eines Migranten zwischen einer Herkunftsgesellschaft und zweier Aufnahmegesellschaften lassen sich grundsätzliche Typen des Migrationsverhaltens unterscheiden. Ausgehend von der Gesellschaft, in die eine Person hineingeboren wird (Herkunftsgesellschaft), soll die erste Abwanderung als Erst-Emigration definiert werden $(A \rightarrow B$; $A \rightarrow C)$. Schließt sich ganz allgemein eine Rückkehr zu einem vorherigen Aufenthaltsort an, dann wird dies in der Literatur üblicherweise mit Remigration $(A \rightarrow B \rightarrow A$; $A \rightarrow C \rightarrow A ; B \rightarrow C \rightarrow B$ ) bezeichnet. Für die Wanderung von einer Aufnahmegesellschaft $(B)$ zur nächsten $(C)$ wird im Folgenden der Begriff Zweit-Emigration $(B \rightarrow C$; 
$\mathrm{C} \rightarrow \mathrm{B})$ verwendet. Emigrationsvorgänge, denen eine Rückkehr vorausging und die daraufhin nicht zu einem vorherigen Emigrationsziel zurückführen, sind somit ZweitEmigrationen mit vorangegangener Remigration $(A \rightarrow B \rightarrow A \rightarrow C ; A \rightarrow C \rightarrow A \rightarrow B)$. Vorstellbar sind darüber hinaus unterschiedliche Kombinationen von Emigrations-Remigrations-Schleifen; um das Schema nicht noch weiter auszudifferenzieren, sollen wiederholte Emigration-Remigrations-Bewegungen zwischen den selben Gesellschaften als Pendelmigration bezeichnet werden $\quad$ ( $z$.B.: $\quad A \rightarrow B \rightarrow A \rightarrow B \rightarrow A$; $\mathrm{A} \rightarrow \mathrm{B} \rightarrow \mathrm{C} \rightarrow \mathrm{B} \rightarrow \mathrm{A} \rightarrow \mathrm{C}$ ).

Daraus folgt beispielsweise für die Analyse von Familienabwanderungen, dass für die Generation der Einwanderer die Rückkehr in die Herkunftsgesellschaft eine Remigration darstellt, während die in die Aufnahmegesellschaft Hineingeborenen erstmals emigrieren. In der Regel werden in den sozialwissenschaftlichen Studien diese Unterscheidungen nicht getroffen und alle Abwanderungen aus der Aufnahmegesellschaft als Remigration interpretiert (Constant/Zimmermann 2003, 3). Oft scheiterten detailliertere Analysen bereits an der unzureichenden Datenerfassung, allerdings gibt es auch Ausnahmen, bei denen entsprechende Unterschiede zum Teil berücksichtigt werden konnten (z.B. Ernst 2002, Nekby 2004). Darüber hinaus verläuft die Erfassung der Zuzüge aus dem Ausland in den meisten Fällen analog zur Abwanderung und so bleibt in der Regel offen, ob es sich um Erst-, Zweit-, Reoder Pendelmigration handelt.

\subsubsection{Umfang der Abwanderung von Immigranten und ihrer Nachkommen und Defizite der Erfassung}

Dirk Hoerder gelangt in seiner historischen Untersuchung der Arbeitsmigration zwischen Nordamerika und Europa zu der Einschätzung, dass die Rückkehr von Emigranten und ihren Nachkommen als Forschungsgegenstand lange Zeit vernachlässigt wurde, obwohl sie einen beträchtlichen Teil der internationalen Wanderungsbewegungen auszumachen scheint (vgl. Hoerder 1985). Leider lässt sich der tatsächliche Umfang meist nicht genau bestimmen, da eine dafür notwendige detaillierte Erfassung der individuellen Migrationsverläufe unterbleibt. Die Auswertung einseitig 
geführter Statistiken im Herkunftsland bringt erhebliche Probleme mit sich, da bei der Erfassung der Rückwanderer oft zwischen ehemaligen Auswanderern, ihren Nachkommen und angeheirateten Personen anderer Herkunft, die bereits die Staatsangehörigkeit erhalten haben, nicht unterschieden wird. Ebenso unklar bleibt in aller Regel, ob aus dem Aufnahmeland abwandernde Personen in das Herkunftsland zurückkehren oder ein anderes Ziel wählen. Für die Bundesrepublik gilt zudem, dass Abwanderungen oft nicht erfasst werden, weil die Abmeldung bei den Einwohnermeldeämtern unterbleibt. Die Defizite bei der korrekten Messung der Remigrationszahlen sind schon seit längerem bekannt und beeinträchtigen die Beschreibung der Migrationsprozesse (vgl. Haug 2001).

Aus den Ergebnissen der historischen Remigrationsforschung kann geschlossen werden, dass Rückwanderungsraten ${ }^{17}$ zwischen $20 \%$ und $40 \%$ keine Seltenheit darstellen (Kortum 1981, 168; Breitenbach 1982, 35-36; Tedebrand 1985, 369; Virtanen 1985, 395, Duleep 1994, 47; Ernst 2002, 157). Verglichen damit liegt das Verhältnis von statistisch erfassten Fortzügen und Zuzügen bei Personen ohne deutsche Staatsangehörigkeit für die Bundesrepublik Deutschland im Zeitraum von 1954 bis 2000 mit etwa 80 \% deutlich höher (vgl. Münz/Ulrich 2000, 28; Beauftragte der Bundesregierung für Migration, Flüchtlinge und Integration 2003, 6). Dieser im Vergleich sehr hohe Wert dürfte zum Teil auf das Rotationsprinzip, die staatliche Rückkehrförderung ${ }^{18}$, Mehrfachzählung von Pendelmigranten ${ }^{19}$, die restriktiven Aufenthaltsbestimmungen ${ }^{20}$ und die Einbürgerungspraxis zurückzuführen sein. Darüber hinaus bleibt insgesamt unklar, ob es sich bei den erfassten Fortzügen um Remigration, Erst-Emigration oder Zweit-Emigration handelt. Für ein vollständiges Bild der Abwanderung von Immigranten und ihren Nachkommen müssten zusätzlich

17 Verhältnis der Remigrationen zu den Emigrationen.

18 Eigentlich handelt es sich um eine Abwanderungsförderung, denn ob der jeweilige Abwanderer in sein Herkunftsland zurückkehrt oder sich in einem Drittstaat niederlässt, hat keinen Einfluss auf die Förderung.

19 Insbesondere scheint die Pendelmigration von größerer Bedeutung zu sein, so finden sich in historischen, qualitativen und auch quantitativen Untersuchungen Hinweise für Pendelmigration (Ernst 2002; Constant/Zimmermann 2003 und 2002; Krumme 2004). Amelie Constant und Klaus F. Zimmermann schätzen auf Basis der Daten des Sozio-oekonomischen Panels, dass $60 \%$ der Immigranten wiederholt emigrierten (Constant/Zimmermann 2003, 23).

20 Kennzeichnend für die Auseinandersetzung mit den Determinanten der Wanderungsentscheidung ist, dass implizit für den jeweiligen Akteur Entscheidungsfreiheit unterstellt wird. Dabei wird insbesondere im deutschen Kontext die Vielzahl von aufenthaltsrechtlichen Bestimmungen ausgeblendet, die eine Abwanderung auferlegen und auf deren Grundlage gegebenenfalls eine Zwangsabschiebung erfolgt. 
noch Deutsche mit und ohne Migrationshintergrund getrennt voneinander betrachtet werden.

\subsubsection{Empirische Ergebnisse zur Abwanderung von Personen mit Migrationshintergrund}

Den Abwanderungsabsichten und dem Abwanderungsverhalten von Personen mit Migrationshintergrund wird in Form der Rückkehrabsichten und der Remigration seit nunmehr rund dreißig Jahren größere Beachtung durch die Forschung geschenkt. Das Interesse an der Rückkehr entwickelte sich in Deutschland vor allem bedingt durch die Verstetigung des Aufenthalts der ehemals als Gastarbeiter angeworbenen ausländischen Arbeitskräfte. Mit dem Anwerbestopp 1973 wurde ein Prozess in Gang gesetzt, der aus Gastarbeitern zum Teil Einwanderer werden ließ. Im Zuge dieser Entwicklung begannen die Arbeitsmigranten in stärkerem Maß ihre Familien in die Bundesrepublik zu holen. Seit es Anfang der 1970er Jahre offensichtlich wurde, dass die Aufenthaltsabsichten und das Remigrationsverhalten nicht mehr in Einklang mit dem Rotationsmodell zu bringen waren, gewannen die Absichten und Beweggründe der Immigranten für Wissenschaft und Politik an Bedeutung. Die in der Mehrzahl deskriptiven Untersuchungen waren zunächst überwiegend den Rückkehrabsichten gewidmet, in den letzten Jahren ist jedoch die Erklärung des Rückkehr- und Abwanderungsverhaltens stärker in den Mittelpunkt des Interesses gerückt.

\subsubsection{Rückkehrabsichten der Immigranten in der Bundesrepublik Deutschland}

In den Studien zu den Rückkehrabsichten der ausländischen Bevölkerung kommt immer wieder zum Ausdruck, dass die Remigration ein wichtiger Bezugspunkt im Leben der Immigranten zu sein scheint (Mehrländer 1987, 60-61; Korte 1990, 240255; Krumme 2004, 150). Rückkehrpläne und tatsächliche Rückkehr können den Ergebnissen zufolge nicht gleichgesetzt werden, so zeigen die Forschungsergeb- 
nisse, dass sich in der Regel die Bleibeabsichten im Verlauf des Aufenthalts verlängern. Als Gründe für das Hinausschieben der Rückwanderung werden finanzielle Motive, die Ausbildung der Kinder und die besseren Lebensbedingungen in Deutschland genannt (Mehrländer 1987, 61; Meis 1993, 70; Werth 1983, 352). Die Ergebnisse zeigen zudem, dass eine höhere Position, ein höheres Nettoeinkommen und Familiennachzug bzw. Familiengründung sich rückkehrhemmend auswirken können (Mehrländer 1987, 61-62; Brecht 1994, 187, 212). Mit steigender Schulbildung und besseren Deutschkenntnissen und dem Vorhandensein von Wohneigentum in Deutschland sinkt die Remigrationsabsicht (Steiner/Velling 1992, 12-13). Darüber hinaus senken temporäre Aufenthaltsperspektiven die Neigung, in Bildung und Deutschkenntnisse zu investieren (vgl. Dustmann 2002).

\subsubsection{Determinanten des Abwanderungs- und Remigrationsverhaltens}

Es liegen mittlerweile eine ganze Reihe von Forschungsergebnissen zu den Determinanten des Abwanderungsverhaltens von Personen mit Migrationshintergrund vor. Im Rahmen der durchgeführten Ereignisdatenanalysen wurde immer eine Vielzahl von Variablen berücksichtigt, von denen in der Regel nur ein Teil signifikante Effekte mit der Abwanderung aufwies. Die Analysen werden deshalb hier nicht in allen Einzelheiten wiedergegeben, der Schwerpunkt soll vornehmlich auf den sich abzeichnenden Gemeinsamkeiten der Ergebnisse liegen.

Schon in den amtlichen Statistiken ist zu erkennen, dass es Unterschiede in den Abwanderungsraten der einzelnen Immigrantengruppen gibt. So variierten die Abwanderungsraten unterschiedlicher Immigrantengruppen in den USA ganz erheblich. Für europäische und nordamerikanische Einwanderer lagen die Werte dabei am höchsten, während insbesondere Immigranten asiatischer Herkunft deutlich seltener abwanderten (Jasso/Rosenzweig 1990; Borjas/Bratsber 1996). In Deutschland wurden auf Basis der Daten des Sozio-oekonomischen Panels ähnliche Unterschiede zwischen Italienern, Griechen, Spaniern, Jugoslawen und Türken festgestellt, wobei für die EU-Bürger deutlich höhere Abwanderungswerte ermittelt wurden 
(Jankowitsch/Klein/Weick, 2000; Constant/Massey 2002; Möser 2005). Lena Nekby findet ebenfalls starke Unterschiede zwischen einzelnen Nationalitäten in den Abwanderungsdaten Schwedens (Nekby 2004).

Im Hinblick auf enge soziale Beziehungen lässt sich feststellen, dass Trennung vom Ehepartner oder den Kindern einen fördernden und Zusammenleben einen reduzierenden Effekt auf die Remigrationswahrscheinlichkeit zu haben scheint, denn zumindest für eine der beiden Variablen konnte in mehreren Analysen ein signifikanter Effekt nachgewiesen werden (Dustmann 1993; Jankowitsch/Klein/Weick, 2000; Haug 2000a; Constant/Massey 2002; Nekby 2004; Möser 2005). Zudem konnte Sonja Haug einen sehr starken Zusammenhang mit vorangegangener Remigration von Haushaltsmitgliedern in ihren Analysen der SOEP-Daten nachweisen (Haug 2000a).

Für die Variablen ökonomischer Erfolg, Vollzeiterwerbstätigkeit und hohes Berufsprestige, die als Messung gelungener strukturelle Assimilation gewertet werden können, wurde fast durchgängig eine remigrationshemmende Wirkung ermittelt (Borjas 1989; Brecht 1994; Velling 1994; Edin/LaLonde/Ålsund 2000; Haug 2000a; Constant/Massey 2002), nur die Analyse schwedischer Daten ergab eine in der Tendenz u-förmige Beziehung (Nekby 2004). Die vom deutschen Gesetzgeber bereitgestellten finanziellen Mittel, um Immigranten zur Abwanderung zu animieren, können als wirkungsvoll angesehen werden (Jankowitsch/Klein/Weick, 2000; Möser 2005). Darüber hinaus scheint das Erreichen des Rentenalters die Wahrscheinlichkeit für eine Abwanderung zu erhöhen (Duleep 1994; Steiner/Velling 1992; Jankowitsch/Klein/Weick, 2000; Möser 2005).

Widersprüchliche Ergebnisse liegen für die Beziehung von Bildung und Remigration vor, während in den USA für mexikanische Immigranten mit Collegeabschluss eine höhere Abwanderungsrate festgestellt werden konnte (Reagan/Olson 2000), ließ sich in deutschen Untersuchungen kein signifikanter Zusammenhang nachweisen (Velling 1994, Jankowitsch/Klein/Weick, 2000; Constant/Massey 2002). In den Analysen schwedischer Daten konnten durchgängig abwanderungsfördernde Effekte von Hochschulabschlüssen nachgewiesen werden (Nekby 2004). Ein nicht allzu sehr überraschender Befund aus mehreren Studien, dass mit steigender Aufent- 
haltsdauer die Abwanderungswahrscheinlichkeit sinkt (Dustmann 1993; Brecht 1994; Duleep 1994; Jankowitsch/Klein/Weick, 2000; Möser 2005), bestätigte sich jedoch in zwei Untersuchungen nicht (Velling 1994; Haug 2000a).

\subsection{Einbürgerung von Immigranten}

Die Einbürgerungszahlen für ethnisch nicht-deutsche Immigranten liegen verglichen mit den Zahlen der letzten 30 Jahre heute erheblich höher. An Hand Abb. 3.3.1a ist zu erkennen, dass der Anstieg zu Beginn der 1990er Jahre einsetzte und im Jahr 2000 ein Niveau von fast 200.000 Einbürgerungen erreicht hatte. Seit diesem Höhepunkt sinken die Zahlen kontinuierlich, liegen aber mit zuletzt 127.153 Einbürgerungen immer noch erheblich über dem Durchschnitt gegen Ende der 90er Jahre. ${ }^{21}$

\section{Abbildung 3.3.1a: Zahl der Einbürgerungen von Ausländern in Deutschland}

1975 - 2004

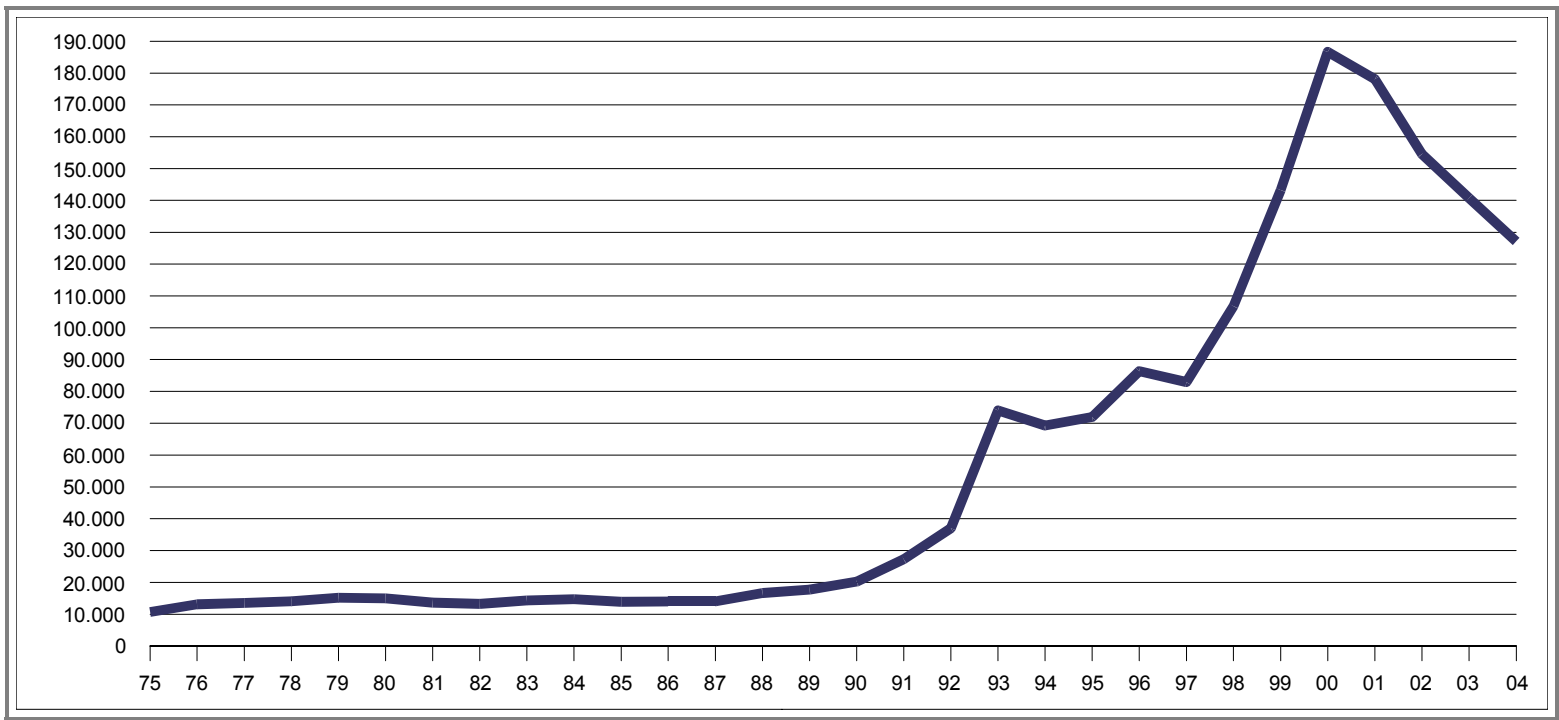

Datenquelle: Statistisches Bundesamt 1997, 2002 und 2005

21 Im Rahmen einer Erhebung unter Personen türkischer Herkunft in NRW gaben rund 25\% der Befragten an, die deutsche Staatsangehörigkeit zu besitzen. Gleichzeitig bestanden bei den bislang nicht eingebürgerten Personen mit Einbürgerungsabsicht zum Teil erhebliche Zweifel, ob sie alle Voraussetzungen für eine Einbürgerung erfüllen (vgl. Zentrum für Türkeistudien 2000). Dies spricht dafür, dass die Interessenten unter den Einbürgerungsberechtigten, die sich selbst hohe Anerkennungschancen einräumten, bereits eingebürgert wurden und deshalb die Einbürgerungszahlen zurückgehen. 
Die schrittweise Ausweitung der Möglichkeit zur Einbürgerung traf offensichtlich auf ein erhebliches Interesse in der ausländischen Bevölkerung. Welche Beweggründe hinter dem Interesse an der Staatsbürgerschaft eines Aufnahmestaates stehen und welche Faktoren einen Einfluss auf die Einbürgerung haben, soll hier mit Hilfe der Forschungsergebnisse zur Einbürgerung näher beleuchtet werden.

Welchen Nutzen versprechen sich die Immigranten davon, einen Einbürgerungsantrag zu stellen? Ganz allgemein sind an die Staatsbürgerschaft in der Regel Rechte und Pflichten gebunden, die im Rahmen einer rechtstaatlichen Ordnung den Staatsbürgern im Unterschied zu den Nicht-Staatsbürgern zustehen bzw. abverlangt werden. Die Einbürgerung ist meist die einzige Möglichkeit, die sich den NichtStaatsbürgern bietet, um in den Genuss der staatsbürgerlichen Rechte zu gelangen. Jenseits der rechtlichen Sphäre werden mit der Staatsangehörigkeit nicht selten Einstellungen und Emotionen verbunden, die eine Einbürgerung als attraktiv oder aber auch als völlig uninteressant erscheinen lassen können. Im Kontext der Integration von Immigranten stellt die Einbürgerung einen Schritt unter vielen im Rahmen der Eingliederung dar, der aber ganz allgemein als Ausdruck eines langfristigeren Interesses hinsichtlich der Aufnahmegesellschaft gewertet werden kann.

Weltweit existieren sehr unterschiedliche gesetzliche Regelungen ${ }^{22}$ und Verfahren der Einbürgerung. Bezogen auf den Personenkreis, der sich einbürgern lassen könnte, fällt allerdings auf, dass nur ein Teil die Möglichkeit zur Einbürgerung nutzt (vgl. Bischoff/Teubner 1991; Zentrum für Türkeistudien 2000; Yang 2002). Daraus ergibt sich die Frage, welche Determinanten haben im Rahmen der nationalen Gegebenheiten einen Einfluss auf die Einbürgerungsneigung und gibt es Einflussgrößen, die unabhängig von den nationalen Besonderheiten wirken.

Die wissenschaftliche Auseinandersetzung mit dem Thema Einbürgerung in Westeuropa ist lange Zeit eher an den rechtlichen Rahmenbedingungen orientiert gewesen, die in enger Verbindung mit dem Konzept der Inkorporation diskutiert wurden (z.B. Brubaker 1992; Castles 1994; Cinar 1994; Martinello 1994; Faist 1995;

22 In der Regel handelt es sich um Voraussetzungen, die der Antragsteller erfüllen muss, um einen Antrag stellen zu dürfen (z.B. fester Wohnsitz im Staatsgebiet) und um Eigenschaften, die eine positive Entscheidung begünstigen oder zwingend erfordert (z.B. Kenntnis der Hauptverkehrssprache, gesichertes Einkommen, Aufgabe einer anderen Staatsangehörigkeit). Einige Staaten, so wie Argentinien und Irland, bieten ihre Staatsangehörigkeit zum Kauf an (vgl. Dornis 2001). 
Jacobson 1996; Halfmann/Bommes 1998; Kronauer 2002; Bloemraad 2003). Seit wenigen Jahren gewinnen auch die Einstellungen und Determinanten der Einbürgerung mehr an Bedeutung.

\subsubsection{Einstellungen zur Einbürgerung}

Erst mit der schrittweisen Ausweitung ${ }^{23}$ der Anspruchseinbürgerung wurde den Einstellungen zur Einbürgerung von ethnisch nicht-deutschen Personen etwas mehr Beachtung zuteil. Mit der Gesetzesänderung von 1993 erhielten vor allem die ehemals als Gastarbeiter eingewanderten ausländischen Mitbürger die Möglichkeit zur Einbürgerung. Die wenigen Analysen zu den Einstellungen standen im Zusammenhang mit Studien zur Fremdenfeindlichkeit, Integration von Immigranten und Integrationsbereitschaft in der Gesellschaft. Es wurden dabei sowohl die Einstellungen der in Deutschland lebenden Ausländer als auch die der Deutschen betrachtet (vgl. Mehrländer/Ascheberg/Uelzhöffer 1996; Kühnel/Leibold 2000; Zentrum für Türkeistudien 2000; Leibold 2004).

Obwohl das Interesse an der deutschen Staatsangehörigkeit bei Immigranten in den MARPLAN Ausländerbefragungen seit 1970 und im Sozio-oekonomischen Panel seit 1984 regelmäßig erhoben wird, liegt bislang keine Analyse zur Entwicklung der Einbürgerungsabsichten vor. In den USA untersuchten Protes und Curtis 1987 die Einbürgerungsabsichten mexikanischer Einwanderer und konnten nur einen Zusammenhang von Hausbesitz, den sie als „taking roots“ interpretieren, mit der Einbürgerungsabsicht feststellen. Mehrländer u.a. stellten in ihrer Untersuchung, die sich auf eine eigene Erhebung aus dem Jahr 1995 bezieht, fest, dass im Durchschnitt weniger als ein Viertel der Immigranten in der Bundesrepublik Einbürgerungsabsichten hegt. Zwischen den betrachteten Nationalitäten ergaben sich erhebliche Unterschiede. Während mit 26,2\% unter den türkischen Befragten das Interes-

23 Das weitgehend am jus-sanguinis orientierte Reichs- und Staatsangehörigkeitsrecht (RuStAG) von 1913 bildete die Grundlage für die Regelung von Einbürgerungsfragen in der Bundesrepublik. 1990 wurde die sogenannte Regeleinbürgerung eingeführt und 1993 wurde den in Deutschland lebenden Ausländern bei Erfüllung bestimmter Voraussetzungen ein Anspruchsrecht auf Einbürgerung eingeräumt. Mit der Novellierung und Umbenennung des RuStAG zum Staatsangehörigkeitsgesetz (StAG) wurde am 01.01.2000 erstmals ein Element des jus-soli in das deutsche Staatsangehörigkeitsrecht aufgenommen (vgl. Dornis 2001). 
se verhältnismäßig am stärksten ausgeprägt war, waren die EU-Bürger (Italiener $12 \%$, Griechen 11,3\%) in erheblich geringerem Umfang an der deutschen Staatsbürgerschaft interessiert. Als wesentliche Gründe für das Interesse wurden rechtliche Vorteile (Aufenthaltsrecht, Wahlrecht und Reisefreiheit in der EU), die persönliche Verwurzelung in Deutschland und die nur noch geringe Bindung an das Herkunftsland genannt. Häufig genannte Gegenargumente waren die Aufgabe der eigenen Staatsangehörigkeit, Rückkehrabsichten und ein ohnehin gesichertes Aufenthaltsrecht (vgl. Mehrländer/Ascheberg/Uelzhöffer 1996). In Befragungen des Zentrums für Türkeistudien unter Personen türkischer Herkunft in Nordrhein-Westfalen, äußerten die befragten türkischen Staatsbürger in ähnlichem Umfang $(25,3 \%$ 1999 und 30,1\% 2000) Interesse an der deutschen Staatsangehörigkeit. ${ }^{24}$ Die am häufigsten genannten Gründe gegen eine Einbürgerung waren Aufgabe der eigenen Staatsangehörigkeit, rechtliche Nachteile in der Türkei, dass keine Vorteile darin gesehen werden, dass man trotzdem Ausländer bleibt und bestehende Rückkehrabsichten. In einer Sekundäranalyse der Städte-Studie ${ }^{25}$ des Instituts für Konflikt- und Gewaltforschung kommt zum Ausdruck, dass die Einbürgerungsabsichten der türkischen Befragten nach Altersgruppen getrennt analysiert starke Unterschiede aufweisen. Unter den jüngeren Personen (16-29 Jahre) äußerte mit 59\% die Mehrheit eine Einbürgerungsabsicht, während nur bei 15\% der älteren Befragten (45 und älter) ein solche Absicht bestand. Die bisher eingehendste Untersuchung der Einbürgerungsabsichten für Deutschland hat Claudia Diehl vorgelegt. Die Analysen der Daten des Integrationssurveys des Bundesinstituts für Bevölkerungsforschung zeigen, dass insbesondere die emotionale Identifikation mit Deutschland, Deutsch als Verkehrssprache unter Freunden, in Deutschland geboren zu sein und geringe Religiosität die Einbürgerungsabsichten von türkischen Befragten erhöhen (Diehl 2002).

Auf Basis der Allgemeinen Bevölkerungsumfrage der Sozialwissenschaften (ALLBUS) 1996 betrachteten Kühnel und Leibold, welche Voraussetzungen von den Befragten für die Einbürgerung von Immigranten als wichtig angesehen wurden. Dabei zeigte es sich, dass die Meinungen von deutschen und ausländischen

24 Allerdings gaben 1999 15,7\% und 2000 24,5\% der Respondenten an, bereits deutsche Staatsangehörige zu sein.

25 Befragt wurden deutsche $(n=929)$ und türkische $(n=708)$ Personen in den Städten Duisburg, Münster und Wuppertal. 
Befragten weitgehend übereinstimmten. Sowohl Deutsche als auch Ausländer legten größten Wert auf strafrechtliche Unbescholtenheit und die Fähigkeit, den eigenen Lebensunterhalt bestreiten zu können. Die Mitgliedschaft in einer christlichen Glaubensgemeinschaft wurde hingegen von allen Gruppen als unwichtig angesehen. Geringe Unterschiede bestanden lediglich in der Bedeutung der Geburt in Deutschland und der deutschen Abstammung. Personen nicht-deutscher Herkunft sahen diese Punkte als weniger wichtig an, während Aussiedler der Geburt in Deutschland keinen hohen Stellenwert einräumten, dafür aber die deutsche Abstammung als bedeutsamer ansahen (vgl. Kühnel/Leibold 2000).

\subsubsection{Determinanten der Einbürgerung}

Die Auseinandersetzung mit den Determinanten im Einbürgerungsprozess findet hierzulande erst seit kurzem statt (Dornis 2001; Diehl 2002; Diehl/Blohm 2002; Özcan 2002), während in klassischen Einwanderungsnationen und insbesondere in den USA schon sehr viel länger und kontinuierlich zu den Hintergründen der Einbürgerung geforscht wird (Bernard 1936; Krassowski 1963; Beijbom 1971; Evans 1988; Yang 1994, 2002). Allerdings dürfte die Einschätzung DeSipios immer noch zutreffen, dass „Naturalization, as a focus of social science research, has not received the attention it merits.“ (DeSipio 1987, 402)

Der Einbürgerungsprozess ist im Regelfall komplexer als die Abwanderung, denn im Rahmen des Einbürgerungsprozesses sind zumindest immer zwei Parteien zu berücksichtigen, die Einfluss auf die Einbürgerungsvorgänge haben. Die Einbürgerungsentscheidungen werden im Wesentlichen von den gesetzlichen Bestimmungen und ihrer Ausgestaltung in der Verwaltungspraxis sowie den ausländischen Personen als Adressaten des Einbürgerungsrechts beeinflusst (vgl. Özcan 2002). Es kann deshalb auch nicht von dem Einbürgerungsverhalten gesprochen werden, weil zumindest ein Verwaltungsbeamter und ein Antragsteller miteinander interagieren. Es gibt demgemäß zwei Forschungsperspektiven, eine am Einbürgerungsrecht und dem Verwaltungshandeln orientierte sowie eine an den Merkmalen der Eingebürgerten ausgerichtete, zu deren Ergebnissen in den folgenden beiden Abschnitten ein kurzer Überblick gegeben werden soll. 


\subsubsection{Verwaltungshandeln und Einbürgerung in der Bundesrepublik Deutschland}

Welchen Einfluss das Verwaltungshandeln auf die Antragstellung und die Einbürgerungsquote hat, lässt sich an Hand der Studie von Christian Dornis erschließen. In der er herausarbeitet, dass neben der Struktur der für die Einbürgerung zuständigen Behörden insbesondere die Prüfung der Einbürgerungsvoraussetzungen und die Informationspolitik zum Einbürgerungsrecht wesentlichen Einfluss auf die Einbürgerungsquote zu haben scheinen. Als Ergebnis der Untersuchung von Einbürgerungsentscheidungen in der Bundesrepublik formuliert der Autor zwei kausale Zusammenhänge: Erstens, je liberaler Einbürgerungsvoraussetzungen (insbesondere Sprachkenntnisse) überprüft werden, desto höher ist die Einbürgerungsquote und zweitens, wenn der gleiche Sachbearbeiter Ausländer- und Einbürgerungsangelegenheiten betreut, dann liegt die Einbürgerungsquote über dem Durchschnitt des Bundeslandes (Dornis 2001, 182).

Anders als Bultmann, der die Unterschiede in den Einbürgerungsquoten der Bundesländer als Ergebnis der restriktiven bzw. liberalen Auslegung der Einbürgerungsvorschriften ansieht, vermutet Dornis die wesentlichen Ursachen auf der Nachfrageseite und dort vor allem in der unterschiedlichen Zusammensetzung der Bevölkerung nach Nationalitäten. Als Beleg für diese These dient die signifikante Korrelation zwischen türkischem Bevölkerungsanteil und der regionalen Einbürgerungsquote, die mit der unter türkischen Immigranten höheren Einbürgerungsabsicht korrespondiert (vgl. vgl. Bultmann 1999; Dornis 2001).

\subsubsection{Determinanten der Einbürgerung nach Eigenschaften der Eingebürgerten}

Inwieweit die Merkmale der Eingebürgerten über eine Einbürgerung entscheiden. ist international seit längerem Forschungsgegenstand; in der Bundesrepublik wird der Thematik erst seit wenigen Jahren wissenschaftliche Aufmerksamkeit zuteil. 
Bereits 1936 konnte Bernard mit seiner Untersuchung us-amerikanischer Daten zeigen, dass Nationalität, Bildung, Einkommen und Status im Zusammenhang mit der Zeit stehen, die bis zur vollständigen Einbürgerung einer Einwandererkohorte verstreicht (Bernard 1936). Der positive Effekt eines hohen sozio-ökonomischen Status' auf die Einbürgerung konnte in weiteren Studien bestätigt werden (Kassowski 1963; Beijbom 1971). Dieser Ansatz wurde dahingehend weiterentwickelt, dass weitere Variablen zur Erklärung der Einbürgerung herangezogen wurden. Die kulturelle und soziale Assimilation der Immigranten standen dabei im Vordergrund, für deren einbürgerungsfördernde Wirkung auch einige empirische Belege vorliegen (vgl. Evans 1988, Liang 1994, Yang 1994, Diehl 2002). Zur Bildung sind die Befunde etwas widersprüchlich. In einigen Studien konnten positive Effekte und in anderen konnte kein Zusammenhang festgestellt werden. Philip Q. Yang ermittelte in seiner Untersuchung japanischer und vietnamesischer Immigranten eine nicht lineare Beziehung zwischen Bildung und Einbürgerung. Personen mit niedrigeren oder höheren Bildungsabschlüssen ließen sich seltener einbürgern als die Befragten mit mittleren Schulabschlüssen (Yang 2002).

Neben der ökonomischen, sozialen und kulturellen Integration der Immigranten wurden auch Merkmale der Herkunft- und Aufnahmegesellschaft zur Erklärung der Einbürgerung herangezogen. Jasso und Rosenzweig konnten Zusammenhänge der Einbürgerungsquoten mit der Alphabetisierungsrate und der ökonomischen Leistungsfähigkeit des Herkunftslandes nachweisen (Jasso/Rosenzweig 1987). Für seine Analyse der Einwanderungskohorten von 1970-1974 berücksichtigte Yang neben Merkmalen des Herkunftslandes auch die kulturelle und ökonomische Assimilation der Einwanderer. Die Ergebnisse belegen eindrucksvoll, dass die Qualität der Englischkenntnisse am meisten zur Erklärung der Einbürgerung beiträgt. Als deutlich weniger wichtig aber signifikant erwiesen sich die meisten berücksichtigten Variablen. Unter anderem besaßen eine einbürgerungsfördernde Wirkung Hausbesitz, höheres Einkommen, längere Schulbildung, minderjährige Kinder im Haushalt, geringere ökonomische Leistungsfähigkeit der Herkunftsgesellschaft, die Mitgliedschaft in den US-Streitkräften, hohe Konzentration der ethnischen Gruppe in Städten, eine größere ethnische Gemeinschaft, Herkunft aus einem sozialistischen Staat und eine höhere Entfernung zwischen Herkunftsland und den USA. Senkend wirkten sich entgegen der Erwartung die Anerkennung doppelter Staatsbürgerschaft 
durch den Herkunftsstaat und Englisch als eine offizielle Sprache im Herkunftsland aus (Yang 1994).

Die spärlichen Forschungsergebnisse zur Erklärung der Einbürgerung aus Deutschland lassen eine Reihe von Ähnlichkeiten erkennen. So bestehen zwischen den einzelnen Nationalitäten erhebliche Unterschiede hinsichtlich ihrer Einbürgerungsquote, zwischen 1995 und 1998 wurden jährlich ca. 2\% der türkischen und nur rund 0,2\% der italienischen Staatsbürger in Deutschland eingebürgert. Die Ergebnisse zur Konzentration der ethnischen Gruppe entsprechen den internationalen Befunden, denn die Stadtstaaten weisen im Bundesvergleich hohe Ausländerkonzentration und gleichzeitig die höchsten Einbürgerungsquoten auf (Dornis 2001).

Diehl kommt in ihrer Analyse der Einbürgerungen italienischer und türkischer Befragter des Integrationssurveys zu dem Ergebnis, dass die kulturelle und identifikative Assimilation in Deutschland erheblichen Einfluss auf die Einbürgerung ausüben. Das Gefühl Deutscher zu sein und eine geringe Religiosität wirken sich für beide Gruppen einbürgerungsfördernd aus. Es bestehen aber auch eine Reihe von Unterschieden zwischen den Gruppen, so ließen sich türkische Frauen signifikant häufiger einbürgern als türkische Männer, hingegen waren bei Italienern keine Geschlechtsunterschiede feststellbar. Der Geschlechtseffekt weist zudem eine Beziehung zum Alter auf, da er vor allem auf den höheren Einbürgerungsraten der 18-35 jährigen Frauen basiert. Für italienische Befragte erwies es sich darüber hinaus als einbürgerungsfördernd, wenn die Personen in Deutschland geboren wurden, die in der Bundesrepublik geborenen italienischen Staatsbürger weisen eine doppelt so hohe Einbürgerungsrate wie die italienischen Immigranten auf (vgl. Diehl 2002).

\subsection{Zwischenfazit zu den Forschungsergebnissen}

Abwanderung und Einbürgerung sind zwei Aspekte, denen bislang von wissenschaftlicher Seite her nicht allzu große Aufmerksamkeit gewidmet wurde, obwohl sie herausragende Ereignisse im Rahmen des Aufenthaltes in einer Aufnahmegesellschaft darstellen. Überdies macht Abwanderung aus ehemaligen Aufnahmegesellschaften einen erheblichen Teil der stattfindenden Migration aus und Einbürge- 
rung ist für klassische, wie neue Einwanderungsländer ein wichtiges politisches Instrument, um über Anerkennung und Gleichstellung Zuwanderer an die Aufnahmegesellschaft zu binden.

In der Forschungsliteratur werden Abwanderung und Einbürgerung getrennt voneinander betrachtet und eher zu Einwanderung und Aufgabe der Staatangehörigkeit in Beziehung gesetzt. Zudem lassen sich noch weitere Unterschiede finden, so zerfällt Abwanderung bei genauerem Hinsehen in unterschiedliche Akte, die - wenn nicht erzwungen - der Entscheidung des Immigranten unterliegen. Hingegen ist Einbürgerung ein Vorgang am Ende einer Interaktionskette zwischen Immigrant und Aufnahmegesellschaft, der ausgehend von Interessen und Angeboten über Anträge und Prüfung bis hin zur Anerkennung verläuft. Trotzdem ergeben sich aus den Forschungsarbeiten Gemeinsamkeiten, die m. E. eine verknüpfte Betrachtungsweise sinnvoll erscheinen lassen.

Entsprechend der dargestellten Forschungsergebnisse sind eine Reihe von Faktoren vorhanden, die in ähnlicher Weise einen Einfluss auf Abwanderung und Einbürgerung ausüben oder mit ihnen im Zusammenhang stehen. Nicht weiter überraschend sind die Unterschiede zwischen einzelnen ethnischen Gruppen bzw. Nationalitäten im Hinblick auf ihre Abwanderungsraten; eben solche Unterschiede finden sich bei der Einbürgerungsabsicht und beim tatsächlichen Wechsel der Staatsangehörigkeit. Sofern Merkmale des Herkunftslandes berücksichtigt wurden konnten Beziehungen zwischen Alphabetisierung, ökonomischer Leistungsfähigkeit und der Entfernung zwischen Herkunfts- und Aufnahmegesellschaft und der Einbürgerung festgestellt werden. ${ }^{26}$ In fast allen Studien ergaben sich auch Zusammenhänge mit der beruflichen Position, dem Einkommen bzw. ökonomischem Erfolg, die als Indiz für wirtschaftliche Integration gewertet werden können. Ganz ähnliche Befunde liegen für familiäre Bindungen, Sprachkenntnisse, Wohneigentum und die emotionale

26 Im Sinne einer Kosten-Nutzen-Abwägung der Einbürgerung muss berücksichtigt werden, dass nicht für jeden Immigranten der selbe Nettonutzen mit einer Einbürgerung verbunden ist. Dem jeweiligen Zugewinn an Rechten (z.B. politische Teilhabe, gesicherter Aufenthaltsstatus, Freizügigkeit in der EU) und der Befreiung von Pflichten (z.B. Wehrdienst im Heimatland) stehen als Kosten neue Pflichten (in Deutschland z.B. Wehrdienst für männliche Personen), der Verlust an zum Teil länderspezifischen Rechten im Heimatland (z.B. türkisches Erbrecht) und die Transaktionskosten selbst (z.B. Gebühren für Ein- und Ausbürgerung und der zeitliche Aufwand) gegenüber. Es ist deshalb auch nicht verwunderlich, dass sich abhängig vom Herkunfts- und Aufnahmeland, aber auch der Emigrationsgründe Unterschiede bezogen auf das Einbürgerungsverlangen einstellen. 
Identifikation mit der Aufnahmegesellschaft vor. Der Verlust von Bindung, wie das Erreichen des Rentenalters oder Familiennachzug, bewirkt eine höhere Wahrscheinlichkeit der Abwendung von der jeweiligen Gesellschaft zu der dadurch die Bindungen gelockert wurden. Insgesamt ergeben sich von ihrer Richtung her betrachtet relativ eindeutige Effekte: Was Abwanderung fördert, senkt die Wahrscheinlichkeit für Einbürgerung und umgekehrt. Es existieren aber auch Merkmale, die keinen eindeutigen Zusammenhang aufweisen, so z.B. sind die Befunde zur Bildung als heterogen zu bezeichnen. Im Sinne des hier charakterisierten Bindungsgedankens stellt Bildung eher ein Potential dar Bindungen zu entwickeln.

Die Gemeinsamkeit von Abwanderung und Einbürgerung liegt im jeweiligen Akt der Hin- und Abwendung, mit dem Bindungen gelöst und neue hergestellt werden. Allerdings stehen sich Einbürgerung und Abwanderung in dieser Hinsicht diametral gegenüber. Die Abwanderung deutet eine geringe Bindung zur Aufnahmegesellschaft an, während Einbürgerung tendenziell als Ausdruck eines längerfristigen Interesses gewertet werden kann. Es spricht deshalb einiges dafür, sie nicht als isolierte Handlungen zu werten. Beide können als Resultat des Integrationsverlaufs aufgefasst werden, das hauptsächlich von den Immigranten und den Bedingungen in der Aufnahmegesellschaft beeinflusst wird. Zusätzlich besitzen tatsächliche und wahrgenommene Veränderungen der Herkunftsgesellschaft wie auch alternativer Migrationsziele Einfluss auf den weiteren Integrationsverlauf.

Das alles zusammengefasst vermittelt den Anschein, dass Integration von Immigranten bezogen auf Abwanderung und Einbürgerung einer Art Selektionslogik folgt, in deren Rahmen die Aufnahmegesellschaft wie ein Filter funktioniert. Ein Teil der Immigranten wird auf Dauer bleiben, während andere nach einem mehr oder weniger langen Aufenthalt abwandern. Zuwanderer, denen es gelingt, vielfältige Bindungen aufzubauen, bleiben mit einer höheren Wahrscheinlichkeit längerfristig in der neuen Heimat und werden in Form der Einbürgerung den bisherigen Bürgern gleichgestellt. Immigranten ohne umfangreiche Bindungen an die Aufnahmegesellschaft werden hingegen sehr viel stärker zur Abwanderung tendieren. ${ }^{27}$ Unklar bleibt dabei jedoch, welche kausalen Beziehungen die einzelnen Integrationsbe-

27 Wenn nicht sogar von Seiten des Aufnahmelandes aktiv Anreize geschaffen werden oder Druck bzw. Zwang ausgeübt wird. 
reiche bzw. Bindungsformen untereinander aufweisen und welche Bedeutung sie für das jeweilige Resultat besitzen. 


\section{Ein handlungstheoretisches Modell zur Erklärung der Abwanderungs- und Einbürgerungsvorgänge auf Basis des Niveaus der Sozialintegration}

Im Rahmen der empirischen Untersuchung soll der Zusammenhang von Integration und dem Auftreten bzw. Ausbleiben von Abwanderung und Einbürgerung analysiert werden. Vor dem Hintergrund der in den vorangegangenen Kapiteln skizzierten Forschungsperspektiven zu den Integrationsverhältnissen und des Forschungsstands in Bezug auf das Verlassen von Aufnahmegesellschaften und der rechtlichen Gleichstellung von Immigranten mit den Bürgern eines Staates kann nun das theoretische Modell entwickelt werden, das als Basis der nachfolgenden empirischen Analysen dienen wird.

Ausgangspunkt ist die Feststellung, dass die von zum Teil starken Differenzen geprägten Forschungsansätze sich letztendlich alle auf die Handlungsstrategien der beteiligten Akteure und auf die sich daran anschließenden typischen Verlaufsmuster der Integration beziehen. Es liegt daher nahe ein Modell heranzuziehen, das explizit auf eine Handlungstheorie Bezug nimmt. Integration sollte dabei aber nicht als eindimensionaler Vorgang definiert sein, sondern eher als ein auf unterschiedlichen Ebenen und teilweise asynchron verlaufender Prozess verstanden werden, der nicht immer auf die Kultur der Mehrheitsgesellschaft ausgerichtet sein muss. Darüber hinaus kann im Anschluss an die dargestellten Forschungsergebnisse festgehalten werden, dass sprachliche Kompetenzen sowie ökonomische, soziale und emotionale Bindungen im engeren Zusammenhang mit Abwanderung und Einbürgerung stehen.

Vor dem Hintergrund der Gastarbeiter-Integration in Deutschland entwickelte Hartmut Esser eine allgemeine Theorie der Assimilation bzw. Sozialintegration von Immigranten, die auf dem methodologischen Individualismus ${ }^{28}$ beruhend eine handlungstheoretische Erklärung der Eingliederung liefert (Esser 1980, 2003). Von zen-

28 Mit dem von Joseph A. Schumpeter (1908) geprägten Begriff des methodologischen Individualismus wird das Postulat verbunden, „daß wir versuchen müssen, alle kollektiven Phänomene als auf Aktionen, Interaktionen, Zielsetzungen, Hoffnungen und Gedanken von Individuen zurückführbar zu verstehen“ (vgl. Popper 1965, 123). 
traler Bedeutung für den Ansatz ist ein Konzept des idealtypischen Verlaufs von Integration in Form der Assimilation, zu der Esser allerdings einschränkend feststellt: „....the classic case of assimilation actually only occurs under quite special conditions“ (Esser 2003a, 20).

Ausgehend vom Entscheidungsprozess für oder gegen assimilative Handlungen wird auch das Auftreten weiterer Phänomene wie z.B. die ethnisch-kulturelle Pluralisierung einer Gesellschaft, ethnische Schichtungen, transnationale Netzwerke und Konflikte zwischen Immigrantengruppen und der Mehrheitsgesellschaft erklärt. Der Assimilationsprozess wird dazu in separate Subdimensionen gegliedert, die wiederum in kausaler Beziehung zueinander stehen. Insgesamt lässt das Modell damit eine Reihe der Charakteristiken erkennen, die Brubaker einem offenen Assimilationsverständnis zuschreibt. Mit dem darauf bezogenen handlungstheoretischen Integrationsansatz soll im empirischen Teil der vorliegenden Arbeit der Versuch unternommen werden, die Abwanderungs- und Einbürgerungsprozesse in der Bundesrepublik zu erklären. Bevor jedoch das eigentliche handlungstheoretische Modell dargestellt werden soll, ist es notwendig, zunächst das grundlegende Konzept der Integration etwas differenzierter zu betrachten.

\subsection{Integration: Von der Makro- zur Mikroebene}

Mit dem Begriff Integration wird üblicherweise das Herstellen eines neuen Ganzen aus einzelnen Teilen oder das Einfügen neuer Teile in ein bereits bestehendes Gefüge bezeichnet. Im Kontext der Immigration kann Integration im Sinne des letzteren Falls ganz allgemein als die Eingliederung von Immigranten in eine Aufnahmegesellschaft definiert werden. Integration ist somit ein Prozess, an dessen Ende ein wie auch immer gearteter Zustand des Integriert-Seins steht.

Hierbei ist es hilfreich, die Ebene der Akteure und die der Gesellschaft zu unterscheiden, denn während eine Gesellschaft hoch integriert sein mag, können gleichzeitig Akteure fast ohne jegliche Anbindung darin existieren. Um diese Unterscheidung zu kennzeichnen, verwendet Esser im Anschluss an David Lockwood den Begriff Systemintegration für den Integrationszustand der Gesellschaft und Sozialinte- 
gration für das Integrationsniveau der Akteure und Gruppen innerhalb der Gesellschaft (vgl. Esser 2001, 3). Das Ausmaß und die Struktur der Systemintegration einer Aufnahmegesellschaft steht im engen Verhältnis zur Sozialintegration der einzelnen Akteure und der durch sie gebildeten Gruppen.

Die Differenzierung in Makro- und Mikroebene der Integration bringt einige Vorteile für die Analyse der Integration in Aufnahmegesellschaften mit sich. Es lassen sich nämlich eine Reihe typischer Integrationszustände ethnisch homogener und heterogener Gesellschaften analytisch unterscheiden. Dabei wird offenkundig, dass hohe Systemintegration nicht pauschal mit einer soliden Sozialintegration gleichgesetzt werden kann und auch umgekehrt gute Sozialintegration der einzelnen Mitglieder nicht immer eine nachhaltige Systemintegration zur Folge hat.

In Abbildung 4.1a sind einige charakteristische Formen von Netzwerken dargestellt, die Kreise stehen dabei für Individuen und die Linien für die unterhaltenen Bezie-

Abbildung 4.1a: Schemata zum Verhältnis von Systemintegration und Sozialintegration in ethnisch homogenen und heterogenen Gesellschaften

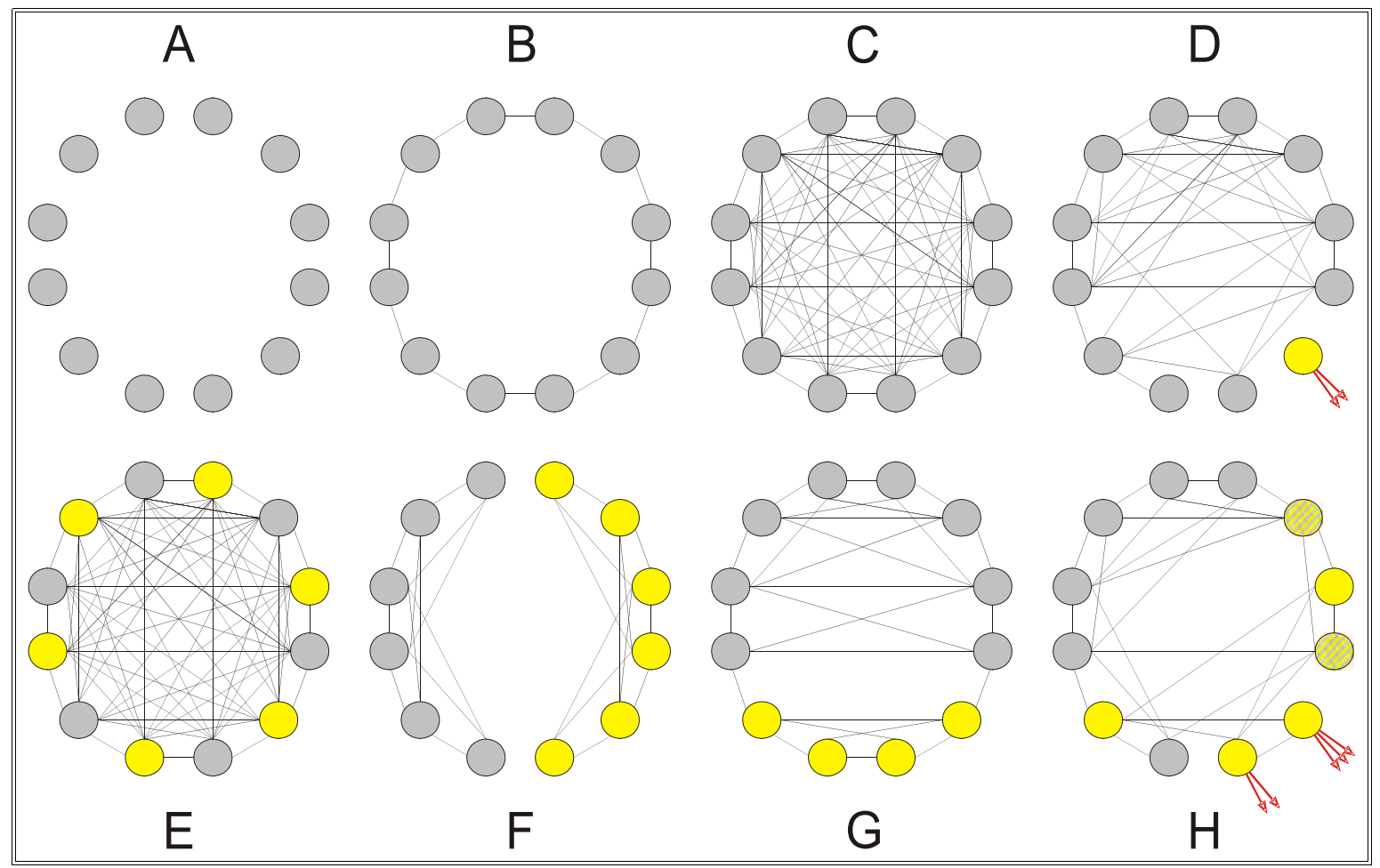


hungen. Die einzelnen Netzwerke veranschaulichen unterschiedliche gesellschaftliche Integrationszustände. Werden die Netzwerke A, B und C verglichen, so ist leicht zu erkennen, dass im ersten Fall keinerlei Sozialintegration vorliegt und somit auch keine Systemintegration gegeben sein kann. Die Varianten B und C unterscheiden sich in der Stärke der Systemintegration, die von den individuellen Unterschieden der jeweiligen Mitglieder getragen werden. Während in Netzwerk B die einzelnen Elemente jeweils nur zwei Verbindungen aufweisen, ist die Systemintegration in C maximal, weil alle Bestandteile voll integriert sind. Netzwerk D steht für den Fall der Immigration in ein bestehendes Netzwerk mit hoher Systemintegration bei gleichzeitig stark unterschiedlicher Sozialintegration der Akteure. Der Zuwanderer unterhält hier zunächst keine Beziehungen zu den Mitgliedern der Aufnahmege-

sellschaft aber noch einige Verbindungen in sein Herkunftsland. Durch die Netzwerke $\mathrm{E}$ bis $\mathrm{H}$ werden verschiedene Integrationszustände ethnisch heterogener Gesellschaften beschrieben. Netzwerk E steht für eine Gesellschaft, in der alle Akteure unabhängig von ihrer ethnischen Zugehörigkeit voll integriert sind und auch keine segregativen Tendenzen erkennen lassen. Variante $F$ hingegen besteht aus zwei separierten Teilnetzwerken, wie sie für eine vollständig segregierte Gesellschaft kennzeichnend sind. Wird die vertikale Anordnung zusätzlich im Sinne einer sozialen Hierarchie interpretiert, so ist in Schema G eine ethnische Schichtung wiedergegeben, die aus zwei fast voneinander separierten Netzwerken besteht, von denen eines dem anderen strikt untergeordnet ist.

In Reinkultur lassen sich die jeweiligen Varianten sicherlich in der Realität nicht finden. Die Prozesse in modernen differenzierten Gesellschaften sind in der Regel nicht ganz so übersichtlich angelegt. Das letzte Schema $(\mathrm{H})$ deutet dementsprechend eine Mischung verschiedener Entwicklungen an, die sich im Kontext ethnisch heterogener Gesellschaften ergeben können: So können die vertikale Anordnung und die Verbindungen zwischen den Teilen sowohl als tendenzielle ethnische Schichtung als auch im Sinne einer Segmentierung interpretiert werden. Darüber hinaus lassen sich Hinweise auf eine beginnende Auflösung der Gruppengrenzen erkennen und ein Teil der Akteure unterhält über das Netzwerk hinausgehende Verbindungen. 
Mechanismen der Systemintegration sieht Esser in Märkten, mit ihren durch die Interessen der Marktteilnehmer strukturierten Abläufen, Organisationen, als vertikale Vermittlungsinstanzen und Medien - etwa Geld oder die sogenannte Interpenetration - die vorauseilende Berücksichtigung der Logik und Informationen anderer gesellschaftlicher Bereiche bei Entscheidungsprozessen. Alle diese angeführten Mechanismen der Systemintegration geben demnach dem Handeln der individuellen und kollektiven Akteure im Sinne einer Rahmung Orientierung und tragen dadurch wesentlich zum Ausmaß und zur Struktur der fortwährenden gesellschaftlichen Integration bei (Esser 2001, 6-8).

\subsubsection{Sozialintegration und ihr Verhältnis zur Assimilation}

Systemintegration ist der Aspekt, dem in den landläufigen Diskussionen um Integration von Immigranten in aller Regel weniger Aufmerksamkeit geschenkt wird. Im Zentrum der Debatten steht die Sozialintegration der Zuwanderer und ihrer Nachkommen. Esser fasst unter dem Begriff der Sozialintegration mehr als nur die direkten sozialen Beziehungen zu anderen Mitgliedern der Gesellschaft zusammen. Es sind insgesamt vier Dimensionen, die nach Esser im Hinblick auf das IntegriertSein der Akteure zu berücksichtigen sind: Kulturation, Platzierung, Interaktion und Identifikation. Diese einzelnen Dimensionen der Sozialintegration, die nicht völlig unabhängig voneinander sind, sondern in einer kausalen Beziehung zueinander stehen, werden als bedeutsam für die ,individuellen Voraussetzungen einer gelingenden Systemintegration“ bezeichnet (ebd., 8).

Die Kulturation ist dabei die grundlegendste Form der Sozialintegration, denn damit ist die Verfügung über kulturelle Fähigkeiten und Informationen gemeint, die für Interaktionen in der (Aufnahme-)Gesellschaft mehr oder weniger als Standard angesehen und häufig vorausgesetzt werden. Das wichtigste Element ist dabei die in der Gesellschaft verwendete Hauptverkehrssprache, über deren Erlernen nicht nur Kommunikationsfähigkeit, sondern z.T. auch kulturelles Wissen und Zusammenhänge vermittelt werden. Von ihrer Definition her ist die Kulturation unabhängig vom Zeitpunkt ein Lernvorgang, der maßgeblich durch die Gelegenheitsstruktur der Um- 
welt mitbestimmt wird (vgl. ebd. 8). Alle weiteren Integrationsformen sind bei fehlender Kulturation nur in Ausnahmefällen möglich.

Mit der Kulturation eng verbunden ist die Platzierung, bei der es sich um die Besetzung gesellschaftlicher Positionen handelt. Die Eingliederung in ein bestehendes soziales Gefüge steht in Abhängigkeit von den Entscheidungen anderer Akteure und damit von deren Interessen und Einstellungen. Esser bezeichnet die Platzierung als eine „Spezialvariante des Marktgeschehens“ (ebd., 9), weil Akteure Angebote machen, auf die unter Umständen ein Nachfrager mit einer Positionsvergabe reagiert. Es kann sich dabei um Lehrer handeln, die eine Empfehlung aussprechen, um Personalchefs, die eine Stelle vergeben, Verwaltungsbeamte, die einem Einbürgerungsantrag stattgeben oder auch um einen Hausbesitzer, der eine Wohnung vermietet. Weil Platzierung nicht selten über die Ausstattung mit allgemeingültigen oft über die Gesellschaft hinausreichenden Kapitalien (z.B. Geld und Bildung) entscheidet, sieht Esser in ihr die nachhaltigste Form der Sozialintegration (vgl. ebd., 9-10).

Unter dem Begriff Interaktion bindet Esser die sozialen Beziehungen zu anderen Mitgliedern der Aufnahmegesellschaft in sein Modell ein. Es sind darunter Beziehungen zu fassen wie sie zwischen Lebensgefährten, Freunden, Nachbarn oder Arbeitskollegen bestehen. Zwar funktionieren sie nach weniger festen Regeln, unterliegen aber zum Teil explizit rechtlichen Regelungen (z.B. Ehe und Nachbarschaft). Ebenso wie im Falle der Kulturation sind auch Gelegenheitsstrukturen interethnischen Zusammentreffens für den Aufbau sozialer Bindungen von ausschlaggebender Bedeutung. In einer stark ethnisch segregierten Gesellschaft, die sich in Nachbarschaften, Schule und im Arbeitsbereich widerspiegelt, ist diese notwendige Voraussetzung nicht gegeben. Eine weitere Voraussetzung für soziale Beziehungen ist insbesondere die Kommunikationsfähigkeit, die im Kontext der Immigration oft von der vorangegangenen erfolgreichen Akkulturation der Immigranten abhängig ist. Darüber hinaus besitzen nach Esser die materiellen Interessen ebenfalls einen gewissen Einfluss auf die Interaktion, und ein Akteur mit hohen Marktchancen ist insofern auch im Rahmen sozialer Beziehungen attraktiver als Interaktionspartner. 
Die Identifikation definiert Esser als Einstellung des Akteurs, sich mit einem sozialen Gefüge als Einheit wahrzunehmen, die in „kollektiven Orientierungen“, wie z.B. Nationalstolz, ihren Ausdruck findet. Gleichzeitig wird aber mittels dreier Formen der Identifikation eine Dimension der Unterstützung des Systems aufgespannt, die von emotionaler Hingabe bis hin zu ohnmächtiger Hinnahme (Deferenzintegration) reicht (ebd., 12). Emotionale Hingabe als stärkste Form der Unterstützung wird durch Identifikation mit dem Kollektiv und die Anerkennung des Wertekanons und starker Solidarität mit anderen Mitgliedern ausgedrückt und bekräftigt. Die schwächste Form der Unterstützung wird als Hinnahme bezeichnet und gliedert sich in Verkettungs- und Deferenzintegration. Während Deferenzintegration Hinnahme auf Grund fehlender Perspektiven einer Veränderung bezeichnet, ist mit dem Begriff der Verkettungsintegration eine Hinnahme des Systems gemeint, die den inneren Konflikt zwischen Ablehnung bei gleichzeitig guter Einbindung und damit materieller und sozialer Vorteile anspricht. Zwischen emotionaler Hingabe und Verkettungsintegration steht der sogenannte Bürgersinn. Hinter diesem Begriff verbirgt sich die Unterstützung einer kollektiven Ordnung im Sinne des rationalen Kalküls: Solange das System subjektiv als wichtig empfundene Freiheiten und Prinzipien sichert, werden kollektive Ansprüche in angemessener Form akzeptiert. Im Unterschied zur Verkettungsintegration ist der Bürgersinn weniger mit materiellen Belohnungen verknüpft, sondern erinnert eher an das Verhältnis von Bürger und Gemeinwesen in Rousseaus Gesellschaftsvertrag ${ }^{29}$ (ebd., 12-15).

Das Integriert-Sein der Zuwanderer in eine Aufnahmegesellschaft bzw. die Mehrheitsgesellschaft wird als Resultat eines Prozesses betrachtet, der mit dem Erwerb kultureller Grundkenntnisse, der Einnahme einer ersten Position und der Kontaktaufnahme zu den Mitgliedern der Aufnahmegesellschaft beginnt und möglicherweise in der Identifikation mit der Mehrheitsgesellschaft mündet. Bleiben die einzelnen Zwischenschritte, wie Platzierung auf gesellschaftlich höher bewerteten Positionen oder Intensivierung der Kontakte zu den autochthonen Mitgliedern der Gesellschaft aus, wird die emotionale Hingabe mit hoher Wahrscheinlichkeit nicht in der beschriebenen Form ausfallen. Ausgehend von diesem Grundkonzept der Einbindung

29 „Was der Mensch durch den Gesellschaftsvertrag verliert, ist seine natürliche Freiheit und sein unbegrenztes Recht auf alles, wonach inn gelüstet und was er erreichen kann; was er erhält, ist die bürgerliche Freiheit (...).“ und „die Verpflichtungen, die uns an den Gesellschaftskörper binden, sind nur deshalb zwingend, weil sie gegenseitig sind (...).“ (Rousseau [1762] 1991; 22, 33) 
differenziert Esser die Sozialintegration der Immigranten nach dem Integriert-Sein in Aufnahme- und Herkunftsgesellschaft (bzw. ethnische Community) und leitet für diesen Fall vier Typen der Sozialintegration ab. Ist der Immigrant integriert in beide Gesellschaften, so liegt eine Mehrfachintegration vor. Das Gegenteil der Mehrfachintegration, also das fehlen jeglicher Einbindung wird im Anschluss an die Arbeiten von Simmel, Park und Stonequist als Marginalität bezeichnet. Bleibt der Immigrant seiner Herkunftsgesellschaft verhaftet oder integriert sich - wenn überhaupt - in eine ethnische Gemeinde, dann spricht Esser von Segmentation. Entwickelt sich diese Form der Sozialintegration zu einem dominierenden Modell, wird auf der Ebene der Systemintegration betrachtet eine ethnisch segmentierte, bzw. eine entsprechend geschichtete Gesellschaft wahrscheinlicher. Integrieren sich die Immigranten hingegen in ihre Aufnahmegesellschaft und geben die Bindungen an ihre Herkunftsgesellschaft bzw. die ethnische Kolonie auf, dann entspricht dies der klassischen Vorstellung der Assimilation eines Immigranten. Assimilation wird dadurch zu einer Option, für die sich abhängig von den Merkmalen und Entscheidungen des Zuwanderers sowie den Gegebenheiten der Umwelt, ganz unterschiedliche Wahrscheinlichkeiten ergeben.

Analog zu den Subdimensionen der Sozialintegration unterscheidet Esser weitergehend einzelne Aspekte der Assimilation, bestehend aus kultureller, struktureller, sozialer und identifikativer Assimilation. Kulturelle Assimilation steht für die Aneignung von kulturellen Kenntnissen, insbesondere der Hauptverkehrssprache; mit struktureller Assimilation wird das Erreichen von Positionen im Bildungs- und Erwerbsbereich verbunden: soziale Assimilation spricht den Kontakt und die Beziehungsmuster zu Angehörigen der Mehrheitsgesellschaft an und die identifikative Komponente die gefühlsmäßige Beziehung zur Mehrheitsgesellschaft. Die Teilaspekte der Assimilation werden nicht als unabhängig voneinander beschrieben, sondern als untereinander in Beziehung stehend (vgl. Abb. 4.1.1a). 


\section{Abbildung 4.1.1a: Kausale Struktur der Assimilation nach Esser (2001) ${ }^{30}$}

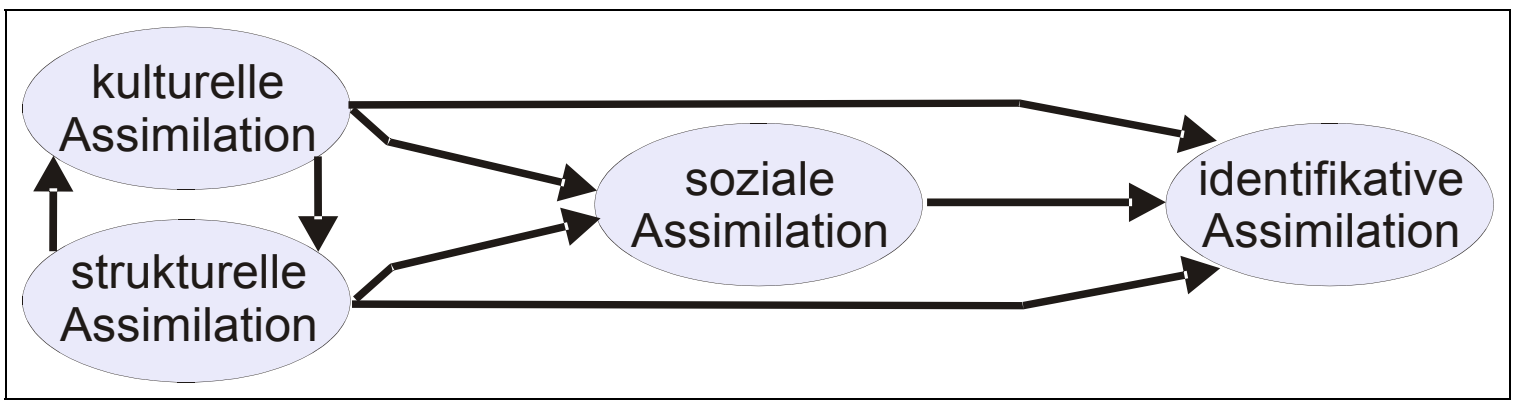

Kulturelle $^{31}$ und strukturelle Assimilation beeinflussen sich gegenseitig und die beiden anderen Aspekte. Die auf der Interaktion beruhende soziale Komponente hat einen Effekt auf die identifikative Assimilation (vgl. ebd. 22).

Das Assimilationsmodell basiert letztlich auf dem Konzept der individuellen Eingliederung von Wanderern und der damit verbundenen Kausalstruktur der Assimilation, das Esser bereits 1980 in „Aspekte der Wanderungssoziologie“ vorgestellt hat (Esser 1980, 225-232). Die vielleicht wichtigste Änderung gegenüber dem älteren Modell sei hier betont, dass nämlich die grundlegende Sozialintegration mit der Einbindung in Herkunfts- und Aufnahmegesellschaft zweidimensional angelegt ist, während der Eingliederungsprozess im früheren Modell zwischen den Polen Assimilation und ethnische Subkultur stattfand (vgl. hierzu auch Esser 1985).

In der Veröffentlichung von 1980 beschreibt Esser an das noch geringfügig anders konzeptualisierte Kausalmodell der Assimilation anknüpfend die Entwicklung in Form eines Prozessmodells der Assimilation, in dem die zeitliche Abfolge der Entwicklung etwas differenzierter gliedert ist (vgl. Esser 1980, 231-234). In das Prozessmodell werden zusätzlich weitere Variablen eingeführt: So wird darin ausgehend vom Grad der Ähnlichkeit des Herkunftssystems zum Aufnahmesystem und der Offenheit des Aufnahmesystems gegenüber den Immigranten der Ablauf bis hin zum Zustand des Assimiliert-Seins über drei Zeitpunkte modelliert (vgl. Abb. 4.1.1b).

30 Die von Esser beschriebenen kausalen Beziehungen der Subdimensionen enthalten keine explizite Aussage zur Unterscheidung von direkten und indirekten Effekten. In der vorliegenden Form wurden die genannten Beziehungen als direkte Effekte interpretiert.

31 In früheren Veröffentlichungen wurde statt des Begriffs kulturell die Bezeichnung kognitiv verwendet. (vgl. Esser 1980) 
Abbildung 4.1.1b: Prozessmodell der Assimilation nach Esser (1980)

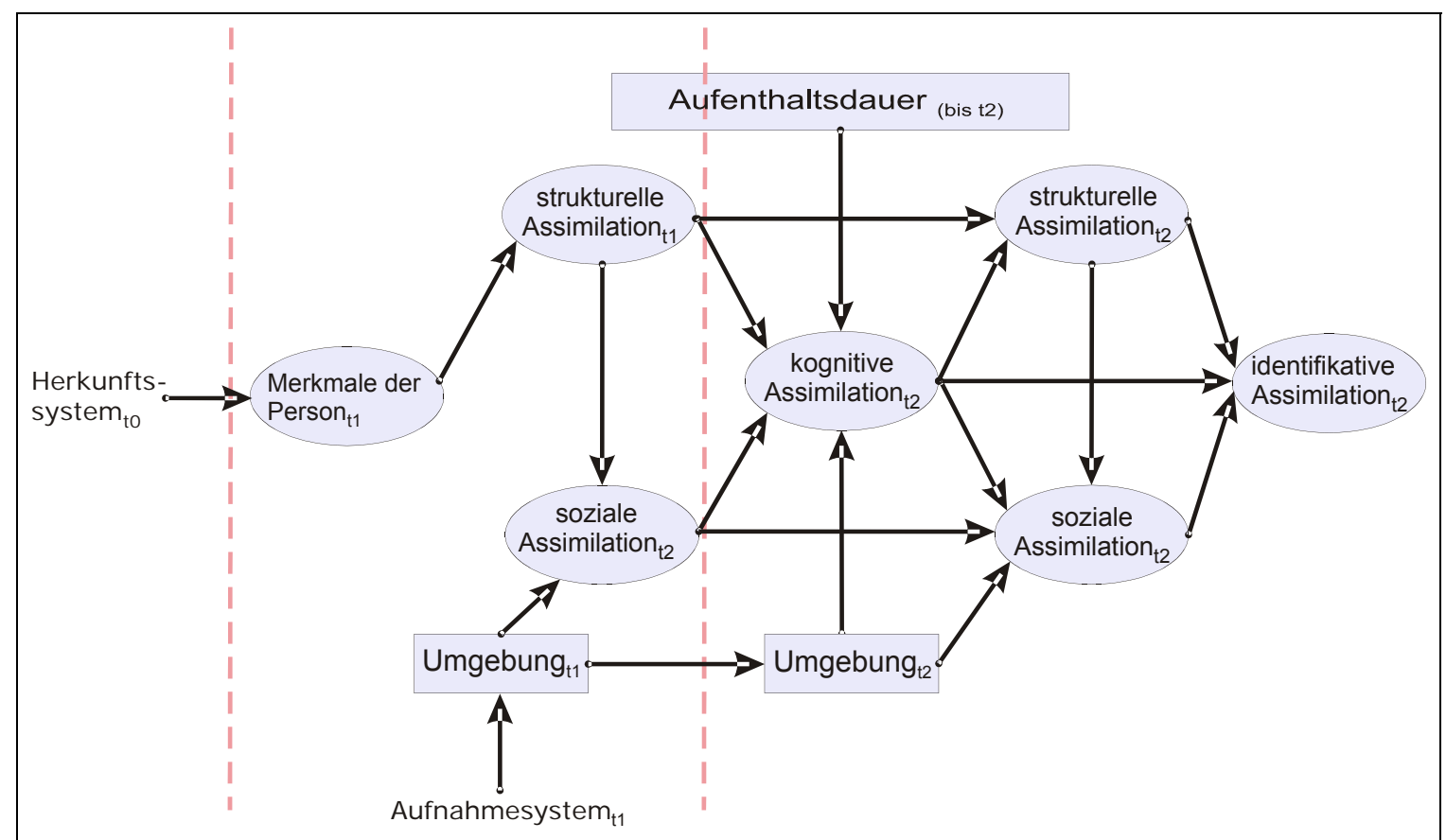

Die erste Phase (t0) ist als Enkulturation oder Primärsozialisation in der Herkunftsgesellschaft zu verstehen, die dem Wanderungsakt voraus geht. Im zweiten Zeitabschnitt, der der Ankunftsphase in der Aufnahmegesellschaft entspricht, beeinflussen Merkmale der Person wie z.B. Bildung, Alter, Geschlecht, Religion, soziale Schicht, Familienstand, Wanderungsmotiv und ethnische Identifikation die erste Positionierung $\left(t_{1}\right)$ im Aufnahmesystem. Diese spezifischen Merkmale werden zu den allgemeinen Variablen Motivation, Kognition, Attribuierung und Widerstand zusammengefasst. Neben der ersten sogenannten strukturellen Assimilation wirkt die soziale Umgebung u.a. über Religion, Bildung, soziale Schicht, Zahl der Mitwanderer, Existenz und Größe ethnischer Kolonien, formale Rechte, Vorurteile, Diskriminierung, Arbeitsmarktsituation auf die Interaktionsmöglichkeiten mit der autochthonen Bevölkerung ein. Ähnlich wie bei den Merkmalen der Person repräsentieren für Esser die Merkmale der sozialen Umwelt allgemeinere Variablen. Diese einzelnen Variablen werden zu den drei Gruppen assimilative Opportunitäten, Barrieren, die einer Assimilation entgegenstehen und nicht-assimilative alternative Opportunitäten zusammengefasst. Esser diskutiert im Anschluss daran die Probleme, die sich aus dieser Form der Modellierung für eine empirische Prüfung ergeben und kommt zu dem Schluss, dass „Auf Variablen, deren Indikatorfunktion nicht eindeutig bestimm- 
bar ist, (wie z.B. bei den beliebten demographischen Variablen), müsste dann freilich verzichtet werden“ (ebd. 220-222). ${ }^{32}$

Soziale und strukturelle Assimilation $z u t_{1}$ bedingen neben der Aufenthaltsdauer und der sozialen Umgebung zu $\mathrm{t}_{2}$ die kulturelle (kognitive) Angleichung ( $\left.\mathrm{t}_{2}\right)$ des Immigranten. Letztere in Verbindung mit der strukturellen Assimilation $\left(t_{1}\right)$ haben einen Einfluss auf die weitere strukturelle Positionierung $\left(\mathrm{t}_{2}\right)$. Auf die soziale Assimilation zum dritten Zeitpunkt üben die Beziehungsmuster zu t1 und die kulturelle Annäherung, das aktuelle soziale Umfeld und die Positionierung einen Einfluss aus. Am Ende steht die emotional-identifikative Angleichung, die durch die kulturelle, strukturelle und nicht zuletzt durch soziale Assimilation bestimmt wird.

Mit dem nach Esser noch bewusst einfach gehaltenen Ansatz liegt ein theoretisch fundiertes Modell vor, das als Basis zur Erklärung des Integrationsprozesses von den Charakteristiken des Herkunftslandes über die Ankunftssituation in der Aufnahmegesellschaft bis hin zum weiteren Eingliederungsprozess dienen kann. Allerdings gesteht Esser ein, dass ,jeder empirische Test des Modells (...) schon erhebungstechnisch und datenanalytisch äußerst aufwendig“ wäre (ebd., 234), denn die Konstrukte sollten gezielt für eine entsprechende Kausalanalyse operationalisiert werden und wären dann retrospektiv oder idealerweise in Form eines Panels über drei Zeitpunkte hinweg zu erheben. Die Problematik der Zuordnung uneindeutiger Variablen diskutiert Esser am Beispiel der Aufenthaltsdauer, die sowohl als Indikator der Assimilation selbst, als auch im Hinblick auf erhaltene assimilative Verstärkung interpretiert werden kann, was dazu führte, dass die Aufenthaltsdauer nicht in das Modell aufgenommen wurde (ders. 1981, 77).

Das Assimilationsmodell von 1980 betont den Gedanken der einseitigen Angleichung an eine Mehrheits- oder Aufnahmegesellschaft, was der oben dargestellten Struktur der Sozialintegration nicht ganz gerecht wird. Die Extreme Assimiliert-Sein, Integration in eine ethnische Gemeinschaft, Mehrfachintegration und Marginalität sowie die möglichen Mischformen sind damit aber nur suboptimal zu differenzieren,

32 Für den Test der Kausalstruktur verwendete Esser 1981 nur auf personaler Ebene Motivation und Kognition. Das Konstrukt Motivation wurde dafür über finanzielle Beweggründe der Wanderung operationalisiert und die Kognition mittels der Variablen Schulbesuch und Schulabschluss im Herkunftsland gemessen. Die Umgebungsvariable wurde bei dieser empirischen Anwendung des Modells nicht berücksichtigt (vgl. Esser 1981, 93-94). 
weil vom Ansatz her eine parallele Bindung zur ethnischen Alternative nicht erfasst wird. Eben diese zweidimensionale Modellierung aber ermöglicht im Rahmen des Bindungskonzepts eine treffendere Beschreibung der auch auf individueller Ebene festzustellenden hybriden Varianten sozialintegrativer Entwicklungen und ihre Auswirkungen auf den Integrationszustand der Gesellschaft insgesamt. Die jeweilige Systemintegration ist dabei, der Logik soziologischer Erklärung folgend, das Explanandum, das sich aus den erwarteten und nicht-intendierten Effekten der individuellen Handlungen aller Akteure im Zusammenhang mit der eigenen und der Sozialintegration anderer ergibt. Im Hinblick auf die Erklärung der Entwicklung hin zur Einbürgerung oder Abwanderung geht es nicht nur um Assimiliert-Sein, sondern um das Niveau der erreichten individuellen Sozialintegration. Zum Beispiel ist eine relativ hohe Positionierung im Erwerbsleben m. E. mit Platzierung treffender zu umschreiben als mit dem Begriff der strukturellen Assimilation, vor allem da die Positionierung auch innerhalb der ethnischen Gemeinschaft erzielt werden kann. Konsequent weiter gedacht, können dissimilative Handlungen sogar zu einer besseren Positionierung führen. Gleichzeitig sollte Assimilation eher mit Einbürgerung und Dissimilation tendenziell mit Abwanderung im Zusammenhang stehen. Ausgehend von diesem Gedanken wird im Rahmen der Erklärung von Einbürgerungs- und Abwanderungsvorgängen der Bezeichnung Integration gegenüber Assimilation der Vorzug gegeben.

Nachdem nun die Beziehungen der Assimilation als Prozess, des IntegriertSeins einzelner Akteure und des Integrationszustands auf gesellschaftlicher Ebene dargestellt sind, drängt sich die Frage auf, welche Mechanismen hinter den Prozessen der Sozialintegration und Assimilation stehen.

\subsection{Das handlungstheoretische Grundmodell}

Wenn die Handlungen der einzelnen Akteure die Grundlage der Sozialintegration darstellen, so erscheint es notwendig, zunächst auf die Hintergründe des Handelns einzugehen, um daran anschließend die Verbindung zum Integrationsniveau des Akteurs herzustellen. Im Hinblick auf die Konzeptualisierung der Entscheidung, die 
der eigentlichen Handlung ${ }^{33}$ zugrunde liegt, ist Esser im Kontext der Integrationsfrage durchgängig an der Theorie der rationalen Wahl orientiert gewesen (vgl. z.B. Esser 1980; 1985; 1988; 1991; 1999a; 2003), wenn er auch darüber hinaus - wie in „Aspekte der Wanderungssoziologie“ - auf andere Theorien und Modelle Bezug nimmt (Esser 1980, 187).

Die Theorie der rationalen Wahl oder Werterwartungstheorie basiert in ihrer allgemeinen Fassung auf den Grundannahmen, dass Menschen mit ihren Handlungen Ziele verfolgen. Um die angestrebten Ziele erreichen zu können, werden Präferenzordnungen auf Basis von Kosten-Nutzen-Kalkulation entwickelt, die dann zur Selektion der Handlung mit dem höchsten Nettonutzen führen. In dieser ursprünglichen aus der neoklassischen Ökonomie stammenden Modellierung des Entscheidungsprozesses wird zusätzlich unterstellt, dass der Mensch, bzw. der homo oeconomicus, auf Grundlage vollkommener Information und der sich daraus ergebenden Erwartungssicherheit sowie ausgestattet mit festen Präferenzen seine Entscheidung nach objektiven Kriterien fällen kann (vgl. Esser 1999b, 236-237). An diesen Modellannahmen wurde im Laufe der Zeit immer wieder Kritik geübt, die zu einer Vielzahl von Varianten und Weiterentwicklungen führte (vgl. Edwards 1992).

Eine dieser Entwicklungslinien ist die Subjectiv Expected Utility Theory (SEU), in der die Basisannahmen dahingehend verändert wurden, dass nicht die in der Situation objektiv gegebenen, sondern die subjektiven Erwartungen zum Tragen kommen und somit Akteure in der selben Situation zu unterschiedlichen Entscheidungen gelangen können (Ramsey 1931; Edwards 1955). Im Prinzip wurde damit die Annahme vollkommener Informiertheit des Akteurs aufgegeben. Das SEU-Modell der Wahlentscheidung ist ebenso, wie das Grundmodell der rationalen Wahl zum Ausgangspunkt neuerer Überlegungen geworden. Insbesondere wurde auf Phänomene wie z.B. Handlungsroutinen, traditionales Handeln, Ambiguitäts-Aversion, Reference-Point-Effekt, Endowment-Effekt und den Sunk-Cost-Effekt, die vom klassischen SEU-Modell nicht zu erklären waren, mit der Entwicklung sogenannter Frame-Selektions-Modelle reagiert, mit denen über die Reduzierung der Kosten für die Informationssuche die Verkürzung eines Entscheidungsprozesses erklärt wird

33 Unter Handeln definierte Esser mit explizitem Bezug zu sozialpsychologischen Konzepten „....alle motorischen und nicht-motorischen Aktivitäten...“ der handelnden Person (Esser 1980, 182). 
(Esser 1990, Lindenberg 1993, Haug 1998). Bei diesen Modellen wird zusätzlich zum SEU-Modell davon ausgegangen, dass bereits Erfahrungen im Hinblick auf Entscheidungssituationen vorliegen und diese als Schemata auf Situation, die als ähnlich wahrgenommen werden, Anwendung finden können (vgl. Esser 1996). Ein Beispiel für Framingprozesse im Integrationskontext sieht Esser in der Entstehung ethnischer Konflikte. Wenn die Rahmung der Beziehungen von Kooperation auf Konflikt umgestellt wird und dadurch Kosten und Risiken einer Auseinandersetzung anders als zuvor bewertet werden, dann wird durch die Neubewertung der Situation eine Mobilisierung der für den Konflikt notwendigen Ressourcen erleichtert (vgl. Esser 1997, 1999).

Der Beschreibung des Entstehens ethnischer Konflikte liegt das von Siegwart Lindenberg entwickelte RREEMM-Modell zu Grunde, dem zufolge Akteure über Ressourcen verfügend und eingeschränkt durch bestehende Restriktionen eine Evaluation der Alternativen vornehmen. Entsprechend der subjektiven Erwartungen im Hinblick auf die Konsequenzen einer Handlung versuchen Menschen dann eine Maximierung ihres Gesamtnutzens zu erzielen (Lindenberg 1985). Der Auswahlprozess kann in drei Schritten modelliert werden: Der erste Schritt besteht in der subjektiven Wahrnehmung der Situation. Es stellt sich für den Akteur die Frage, welche Eigenschaften der Situation handlungsrelevant sind. Diese Eigenschaften lassen sich analytisch in potentielle Ressourcen und Restriktionen bezogen auf die Handlungsrealisierung sowie bestehende Handlungsalternativen unterteilen. Darauf hin erfolgt im nächsten Schritt die Bewertung der antizipierten Konsequenzen der jeweiligen Handlung. In Abhängigkeit der angestrebten Ziele werden die Konsequenzen und damit die Handlungsalternativen in Form einer Präferenzordnung sortiert und mit einer subjektiven Eintrittswahrscheinlichkeit verbunden. Aus dieser Verknüpfung ergibt sich jeweils ein subjektiv erwarteter Nutzen oder Erwartungswert für die berücksichtigten Handlungen. Auf Grundlage dieser Bilanzierung wird dann im letzten Teilschritt eine Handlungsalternative ausgewählt, nämlich jene, deren Erwartungswert am höchsten eingeschätzt worden ist. Für die Erreichung von Zielen müssen Ressourcen aufgewendet werden, die aber in der Regel knappe Güter darstellen und zum Teil erst erworben oder produziert werden müssen, wofür wiederum andere Ressourcen aufzuwenden sind. 


\subsection{Handlungsentscheidungen vor dem Hintergrund der sozialen Produktionsfunktionen}

Die Maximierung des pekuniären Nettonutzens blieb in den ökonomisch orientierten SEU-Modellen zumindest das Hauptziel des (wirtschaftlichen) Handelns. In der sozialwissenschaftlichen Auseinandersetzung mit den SEU-Modellen wurden im Zusammenhang von Handeln und sozialem Kontext andere Zielsetzungen in die Modelle eingeführt (z.B. Becker 1975, Wippler 1987, Lindenberg 1989). Esser greift hierzu die Arbeiten Siegwart Lindenbergs auf, der als Hauptziele menschlichen Handelns physisches Wohlbefinden und soziale Anerkennung postuliert (Esser 1980, 1997, 2003).

Wie gelingt es nun aber dem Einzelnen diese Ziele zu verwirklichen? Das Konzept der sozialen Produktionsfunktionen ist der Versuch, die Frage nach den Zielen in Abhängigkeit von der Einbettung in einen sozialen Kontext zu beantworten.

Um die von Lindenberg postulierten Hauptziele verfolgen und erreichen zu können, bedarf es der Verfügung über Ressourcen oder sogenannte primäre Zwischengüter, auf die das Streben der Akteure deshalb ausgerichtet ist. Der Wert eines spezifischen Zwischenguts leitet sich davon ab, wie effektiv damit Wohlbefinden und Wertschätzung in einer Gesellschaft produziert werden können. Dabei ist es zunächst zweitrangig, was als Zwischengut fungiert; es kann sich dabei z.B. um Geld, Besitz, Bildung, Reputation oder aber auch um askriptive Merkmale handeln. Allerdings sind diese Ressourcen in einer Gesellschaft nicht gleich verteilt, so dass den einzelnen Akteuren zum Teil unterschiedliche und jenen mit schlechter Ressourcenausstattung nicht selten deutlich weniger Handlungsalternativen offen stehen, denn in der Regel müssen Ressourcen aufgewendet werden, um die präferierten Zwischengüter zu erlangen. Die Wahl wird zwar im Sinne der Werterwartungstheorie auf Basis derselben Nutzenfunktion getroffen, die Produktionsfunktionen aber unterscheiden sich bedingt durch die jeweilige Ressourcenausstattung und die gegebenen Opportunitätsstrukturen. Über diesen Mechanismus lassen sich unter anderem die Entstehung unterschiedlicher Präferenzordnungen in sozialen Gruppen erklären. Im Kontext des Kontakts zwischen ethnischen Gruppen kann dieser Aspekt deutlich an Schärfe und Gewicht gewinnen. Denn es besteht die 
Möglichkeit, dass in einer Aufnahmegesellschaft und einer eingewanderten Gruppe unterschiedliche Vorstellungen dahingehend bestehen, was als Zwischengut anerkannt wird und gegebenenfalls auch welcher Wert diesem beizumessen ist.

Für den einzelnen Akteur ergibt sich daraus die Frage, welche Zwischengüter angestrebt werden sollten, die vom ethnischen Umfeld präferierten oder aber jene der Mehrheitsgesellschaft. ${ }^{34}$ Die für den ethnischen Kontext spezifischen Güter mögen in solchen Fällen leichter zugänglich sein, sind aber im Kontext des Aufnahmelandes oft weniger effektiv. Esser leitet aus dieser Feststellung ein objektives Interesse der Immigranten an den effektiveren - in der Aufnahmegesellschaft hoch bewerteten - primären Zwischengütern ab. Diese Zwischengüter sind aber oft nur über assimilative Handlungen erreichbar und so kann es notwendig erscheinen, z.B. die Hauptverkehrssprache des Aufnahmelandes zu erlernen oder einen formalen Bildungsabschluss zu erwerben (vgl. Esser 2003b, 11). Diese Vorgehensweise stellt eine Anpassung an die sozialen und kulturellen Gegebenheiten der Aufnahmegesellschaft durch den Immigranten dar. Aus dem Ansatz der sozialen Produktionsfunktion lässt sich noch eine weitere Strategie ableiten, die ebenfalls tendenziell auf eine ethnische Homogenisierung der Gesellschaft hinausläuft. Die Zugewanderten können die eigenen Kosten für primäre Zwischengüter reduzieren, indem sie die Anerkennung des Wertes eines ihrer spezifischen Zwischengüter in der Aufnahmegesellschaft durchsetzen. In diesem Fall findet aus Sicht der Immigranten eine Anpassung der sozialen Umwelt an ihre Bedürfnisse statt.

Die Logik sozialer Produktionsfunktionen setzt eine zumindest rudimentäre Sozialintegration des Handelnden in die Gesellschaft voraus. Die Integration eines Immigranten kann in die Aufnahmegesellschaft hinein oder in Richtung eines ethnischen Segments erfolgen. Doch generell gilt: Je geringer die Sozialintegration in Bezug zu einer sozialen Gruppe ausgeprägt ist, umso schwerer sollte es dem einzelnen Akteur in diesem Kontext fallen, adäquat und dauerhaft physisches Wohlbefinden und soziale Anerkennung zu erlangen.

34 Günstiger sind selbstverständlich jene Zwischengüter, die in beiden Gruppen Anerkennung finden, allerdings ist für diese auch mit höherem Konkurrenzdruck zu rechnen. 


\subsection{Individuelle Konsequenzen und die Summe der Handlungen}

In den vorangegangenen Abschnitten dieses Kapitels wurde die Bindung der Sozialintegration an Entscheidungen und Handlungen der Akteure herausgearbeitet, mit dem RREEMM-Modell eine am SEU-Ansatz orientierte Handlungstheorie dargestellt sowie die Bindung der sozialen Produktionsfunktionen an die soziale Gemeinschaft und vermittelt darüber an die Sozialintegration aufgezeigt. Im Rahmen dieser Darstellung sind eine Reihe von Begriffen geklärt und bereits einige Zusammenhänge referiert worden; über das Grundmodell soziologischer Erklärung sollen nun die einzelnen Aspekte zusammengeführt werden, um dann, mit Blick auf die strukturellen Konsequenzen, die Verbindung von handlungstheoretisch fundiertem Modell der Sozialintegration zu den Abwanderungs- und Einbürgerungsvorgängen zu erläutern (vgl. Esser 1999b, 91-102).

Es soll nun vor dem dargelegten theoretischen Hintergrund am Prozess der Sozialintegration verdeutlicht werden, wie aus der unüberschaubaren Zahl alltäglicher Entscheidungen die Integration einer Gruppe in ein soziales Gefüge entstehen kann oder Loslösung von einer sozialen Umwelt resultiert.

\subsubsection{Entscheidungen zwischen Assimilation und Differenz}

Jeder neu eingereiste Immigrant ist vor die Aufgabe gestellt, innerhalb der Aufnahmegesellschaft seine Bedürfnisse angemessen zu befriedigen. Vor dem Hintergrund bestehender Restriktionen und Opportunitäten kann er sich zwischen Handlungsalternativen entscheiden. Das gewählte RREEMM-Modell - ergänzt durch den Gedanken der Frame-Selektion - unterstellt, dass die Entscheidung für eine der wahrgenommenen Alternativen letztlich nach rationalen Prinzipien gefällt wird.

Die soziale Situation des Neu-Einwanderers ist von einer ganz spezifischen Logik der Situation geprägt: Mit jeder Handlung in Richtung Assimilation werden die Möglichkeiten verbessert, in der Aufnahmegesellschaft primäre Zwischengüter zu erlangen, allerdings kann dieser Weg sehr langwierig und steinig sein und bedarf 
oft mehrerer Generationen bis zur vollständigen Angleichung. In vielen gesellschaftlichen Bereichen ist eine tendenzielle Assimilation nahezu unvermeidbar. Insbesondere die nachfolgenden Generationen, die einen Teil oder ihre gesamte Schulzeit in einem Aufnahmeland verbringen, erfahren eine En- oder intensivere Akkulturation und erzielen im Vergleich zu ihrer Elterngeneration oft eine Verbesserung bezüglich ihrer Platzierung in der Aufnahmegesellschaft.

Für die Generation an Immigranten, die als Erwachsene kommen und jene der Nachgeborenen, die stark in eine ethnische Gemeinschaft eingebunden sind, kann eine grundsätzlich andere Logik in ihrer Lebenswirklichkeit vorherrschend sein. Unter diesen Umständen mag die Sozialintegration über ethnische Netzwerke oder transnationale Verbindungen nicht nur ertragreicher sein, sondern wird möglicherweise auch als befriedigender erlebt. Dieser Zusammenhang ist nicht zuletzt deshalb gegeben, weil ein größerer Teil der persönlich-ethnischen Ressourcen weiter genutzt werden kann. Die erlebte Befriedigung ist auch dadurch begründet, dass unter Einsatz persönlicher Fähigkeiten etwas geleistet werden kann, was oft unmittelbar mit einer positiven Wirkung auf das Selbstwertgefühl verbunden ist und was auch soziale Anerkennung einbringen kann. Selbst der Aufbau eines ethnischen Netzwerks kann unter diesem Gesichtspunkt als lohnendes Ziel empfunden werden.

Entsprechend der Logik der Selektion sind vorhandene ethnische Netzwerke eine günstige Option und mit jeder Entscheidung für spezifisch-ethnische Güter, bei gleichzeitigem Verzicht auf Anpassung an die Aufnahmegesellschaft, verstärkt sich die Sozialintegration über die ethnische Gemeinschaft. Darüber hinaus gibt es noch einen weiteren wichtigen Faktor, warum gesellschaftliche Integration über ein ethnisches Netzwerk als günstig bewertet werden kann: Es ist, wie in Kap. 3 dargestellt, kein ungewöhnlicher Befund, wenn eine große Zahl der Immigranten über lange Zeit am Plan der Remigration festhält. Spezifisches Kapital, dessen weitere Verwendbarkeit an die Aufnahmegesellschaft gebunden ist, verliert im Fall der Rückkehr seinen Wert, während ethnische Kapitalien im Herkunftsland eher noch von Nutzen sein können. Insofern die genannten Annahmen zutreffen, wirkte das lange Festhalten der staatlichen Seite in Deutschland am Gastarbeitermodell als zusätzlicher Anreiz, die Differenz zur Aufnahmegesellschaft zumindest beizubehalten und 
von tiefgreifenden assimilativen Handlungen abzusehen. Die beschriebenen Prozesse beziehen sich alle auf die individuellen Entscheidungen und Resultate, aber wie wirkt sich die Summe der Entscheidungen auf der Makroebene aus?

\subsubsection{Die Aggregatebene: Veränderungen und deren Auswirkungen}

Assimilation wurde bisher als Angleichungsprozess und die Beibehaltung bzw. der Ausbau von Differenzen als Dissimilation der Individuen verstanden. In diesem Abschnitt soll nun die Assimilation bzw. Dissimilation von Gruppen und die Ergebnisse dieser Prozesse betrachtet werden. Die Frage nach den Auswirkungen auf der Aggregatebene erscheint zunächst einmal banal: Assimilation der Individuen führt zu einer Angleichung der Gruppenmerkmale, während Aufrechterhaltung und Ausbau der Differenz das Gegenteil zur Folge haben. Dies gilt aber nur insofern, als der Assimilationsprozess auf der individuellen Ebene eindimensional gedacht und weiterhin angenommen wird, eine Immigrantencommunity oder eine ethnische Minderheit würde in homogener Form in diesen Angleichungsprozess eintreten.

Aus Gründen der Vereinfachung sollen hier nur zwei Gruppen betrachtet werden, die in Kontakt miteinander treten und sich hinsichtlich der Größe unterscheiden. Schon bei der Berücksichtigung der internen Heterogenität der Gruppen sind auf der Aggregatebene erheblich komplexere Verläufe vorstellbar. Zum einen können sich die Gruppen hinsichtlich der Varianz eines Merkmals unterscheiden und zum anderen können auch im Verlauf der Zeit innerhalb der Gruppen Veränderungen auftreten. Diese Unterschiede und Entwicklungen gehen dann in Kombination mit den Assimilations- und Dissimilationssprozessen der Gruppen einher. In Abbildung 4.4.2a sind einige Entwicklungsverläufe zu drei aufeinanderfolgenden Zeitpunkten (to-t2) skizziert, von denen die Varianten A und B für sehr einfache Verläufe stehen, nämlich die vollständige Stabilität der Merkmalsverteilung über die Zeit. ${ }^{35} \mathrm{Im}$ Falle von $A$ bestehen zwischen Mehrheit und Minderheit keine Unterschiede hinsichtlich des betrachteten Merkmals. Bezogen auf die Mehrzahl biologischer Merkmale dürf-

35 Auch Normalverteilungen als Darstellungsform sind bereits eine grobe Vereinfachung und entsprechen wahrscheinlich nur in seltenen Fällen der Realität. 
te diese Variante die Regel sein, während bei sozialen und kulturellen Eigenschaften seltener mit diesem Muster zu rechnen ist.

Abbildung 4.4.2a: Beispiele für Assimilation und Dissimilation der Aggregate

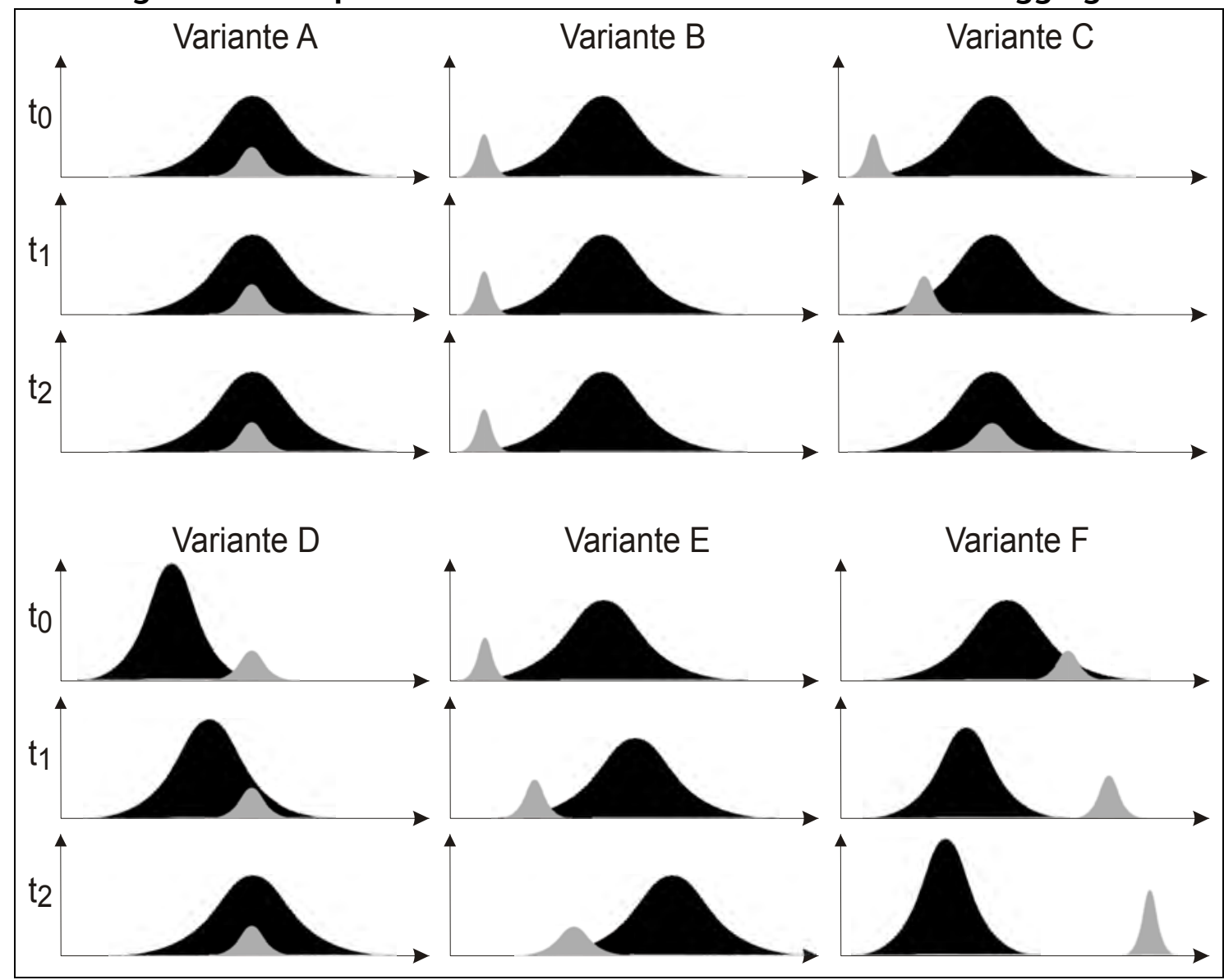

Variante B ist davon gekennzeichnet, dass vorhandene Differenz keine Veränderung erfährt. Bezogen auf Fähigkeiten, Einstellungen, Meinungen und Präferenzen der Individuen sind entsprechende Entwicklungen eher selten, jedenfalls im Vergleich zu biologischen Merkmalen (z.B. Pigmentierung der Haut), die im Kontext der Integration von Immigranten durchaus Bedeutung erlangen können und sich in der Regel als stabiler erweisen. Der Verlauf einer Annäherung nach der Vorstellung des klassischen Assimilationsverständnisses ist unter C dargestellt. Eine tendenziell kleine homogene Gruppe differenziert sich intern und gleichzeitig verschwinden die Unterschiede zwischen den Gruppen im Zeitverlauf. Der von den Gruppen her umgekehrte Verlauf ist dann in Variante D verzeichnet. In Variante E kommt es zwar 
zu einer Veränderung der Merkmalsausprägungen in beiden Gruppen, wobei innerhalb der kleineren auch die Varianz ansteigt, aber der relative Abstand bleibt nahezu gleich. Nur in Variante F ist eine dissimilative Entwicklung dargestellt, bei der zu to noch eine hohe Übereinstimmung zwischen den Gruppen und intern größere Varianz vorliegen, aber zu t2 starke Gruppenunterschiede und hohe Homogenität intern zu verzeichnen sind.

Welche Bedeutung den einzelnen Entwicklungen im Falle von Differenzen zwischen den Gruppen beigemessen wird, ist von der Bewertung des Sachverhalts und dem Verhältnis zu den jeweiligen Zielen im Zusammenhang mit der Integration abhängig. Wenn sich beispielsweise nach 30 Jahren keine Annäherung hinsichtlich der Hautfarbe ergeben hätte, dann wäre das im Sinne des Grundgesetzes völlig belanglos. Würden aber im gleichen Zeitraum die sprachlichen Kenntnisse in der Hauptverkehrssprache sich gemäß Variante B und nicht nach Variante C entwickeln, dann wäre dies bezogen auf eine gute Sozialintegration als problematischer Befund anzusehen. Prozesse der Annäherung, wie unter D charakterisiert, sind zwar wünschenswert, finden aber relativ selten, z.B. im Rahmen einer tendenziellen Überschichtung durch eine zuwandernde Minderheit, statt. Weitere Beispiele lassen sich möglicherweise im Hinblick auf den kulinarischen und kulturellen Austausch finden, wo nicht selten eine größere Offenheit besteht. Der unter E verzeichnete Prozess ist davon gekennzeichnet, dass beide Gruppen sich gleichermaßen entwickeln aber unterschiedliche Ausgangspositionen aufweisen. Beispiele für solche Entwicklungen sind im Bildungsbereich denkbar, wenn es zwar in beiden Gruppen zu durchschnittlich höheren Abschlüssen kommt, der relative Abstand aber weitgehend konstant bleibt (vgl. Kalter/Granato 2004). Allein der Spaltungsprozess in Variante $\mathrm{F}$ ist an sich als problematisch zu bewerten, insbesondere wenn dahinter eine intendierte Differenzierung steht. Die Akzeptanz von Merkmalen, die zu Symbolen der Abgrenzungen stilisiert werden, wie möglicherweise das von einem Teil der Musliminnen getragene Kopftuch, kann auf Dauer einen solchen Verlauf nehmen.

Aus den hier exemplarisch aufgeführten Verlaufsmustern wird deutlich, dass nicht nur eine simple Assimilation stattfindet, sondern dass sehr viel mehr Dynamik in den Entwicklungen stecken kann. Wenn dann noch davon ausgegangen wird, dass es sich um eine mehrdimensional angelegte Entwicklung handelt, bei der ein- 
zelne Prozesse distinkt oder miteinander verknüpft sein können, ist bei aller Vereinfachung vielleicht die Komplexität der realen Vorgänge zu erahnen.

Nun stellt sich die Frage, welche Sachverhalte sind wesentlich und nach welchem Maßstab soll eine Wertung der einzelnen Entwicklungen vorgenommen werden? Vor dem Hintergrund des in Kap. 4.1 dargestellten Integrationsverständnisses ist es unter dem normativen Gesichtspunkt einer breiten und soliden Sozialintegration möglich, eine Bewertung der Tendenzen als vernachlässigbar, begrüßenswert oder problematisch vorzunehmen. Aus diesem Blickwinkel sind vor allem anderen eine grundlegende Kulturation und Platzierung notwendig, um einer ökonomischen und damit sozialen Spaltung vorzubeugen. Im Sinne der sozialen Produktionsfunktionen bedeutet dies: unterlassen es Immigranten oder Mitglieder einer ethnischen Gruppe über einen längeren Zeitraum in die Zwischengüter der Aufnahme- bzw. Mehrheitsgesellschaft $z u$ investieren, dann ist damit langfristig ein Effizienzverlust verbunden. ${ }^{36}$ In der Tendenz führt im Rahmen der Sozialintegration das Aufrechterhalten bzw. der Ausbau von Differenz in größerem Umfang zu ethnischen Spaltungen.

Spaltungsprozesse im Bereich der Kulturation und Platzierung, gleichgültig auf welche Ursache sie zurückzuführen sind, bewirken dem Modell zufolge vertikale Ungleichheit. Ethnische Spaltung korrespondiert mit dem zweiten Teil des Modells der Sozialintegration, denn Interaktion und Identifikation sind die Basis einer Gemeinschaft. In ethnische Comunities besitzen insbesondere Interaktion und Idendtifikation einen hohen Wert, da sie den Zusammenhalt sichern. Horizontale und vertikale Ungleichheit, selbst wenn sie einer realen Basis entbehren und nur auf Zuschreibungen basieren, stellen ein Konfliktpotential in Gesellschaften dar, das sich entlang ethnischer und kultureller Spaltungslinien oft als mobilisierbar erwiesen hat. $^{37}$

36 Nur wenn Gleichwertigkeit zur Aufnahme- oder Mehrheitsgesellschaft vorliegen oder Überschichtung stattgefunden hat, ist Differenz längerfristig weniger problematisch für die Immigranten. Allerdings besteht in solchen Fällen die Gefahr, dass sich Ressentiments unter den Autochthonen bzw. der Mehrheitsbevölkerung ausbreiten.

37 Beispiele für ähnliche Prozesse lassen sich im Rahmen der Judenverfolgung in Deutschland, der Rassentrennung in den USA ebenso, wie im Falle der Separationstendenzen in Jugoslawien nach der Tito-Ära und im kanadischen Quebec finden. 
Im Hinblick auf Abwanderung und Einbürgerung bleibt noch anzumerken, dass beide Vorgänge eine homogenisierende Wirkung entfalten können. Abwanderung von Personen mit geringer und geringster Sozialintegration in Bezug zur Mehrheitsgesellschaft verringert das Ausmaß vorhandener Spaltungen. Dadurch wird das bestehende Konfliktpotential reduziert, weil zum einen die Zahl der Akteure sinkt, die für eine Austragung des Konflikts zur Verfügung steht, und zum anderen die Differenz zwischen Mehrheit und Minderheit tendenziell verringert wird. Abwanderungsförderung bis hin zur gewaltsamen Abschiebung sind denn auch politische Instrumente, die eingesetzt werden, um sich der entstandenen sozialen Probleme im Inneren einer Gesellschaft zu entledigen.

Einbürgerung kann ebenfalls als Strategie der Auseinandersetzung mit Spaltungslinien genutzt werden, die zu ähnlichen Ergebnissen wie die Abwanderungsförderung führt. Die Vergabe der Staatsangehörigkeit folgt nicht nur in der Bundesrepublik dem Schema des Creamings, wonach jene, die eine starke Sozialintegration aufweisen oder zumindest Anlass für ein positive Prognose geben eher in den Kreis der Staatsbürger eingelassen werden. Zwar wurden unlängst Elemente des Geburtsrechts in das Staatsangehörigkeitsrecht aufgenommen, aber die Grundtendenz blieb erhalten. Ein Effekt der Einbürgerung ist die mehr oder weniger starke Reduzierung der Homogenität zwischen den Staatsbürgern. Mit Einbürgerung demonstriert die Mehrheitsgesellschaft aber auch Offenheit gegenüber den Immigranten und gleichzeitig verlieren ethnisch-staatsbürgerliche Spaltungslinien an Eindeutigkeit. 


\subsubsection{Abwanderung und Einbürgerung als Resultat der Sozialintegration}

In diesem Abschnitt soll nun die Verbindung von individueller Sozialintegration als erklärendem Konstrukt und abhängiger Variable, die aus Abwanderung und Einbürgerung gebildet wird, auf Grundlage der theoretischen Vorarbeiten expliziert werden. Hierzu werden Abwanderung und Einbürgerung als Ausdruck einer Bindungsdimension interpretiert. Grundgedanke der Beziehung zwischen Erklärungsmodell und der erklärten Bindung ist, dass durch Sozialintegration kulturelle, strukturelle, soziale und emotionale Bindungen einer Person mit Migrationshintergrund an die Aufnahme- bzw. Mehrheitsgesellschaft entstehen. Dem gemäß sollten Abwanderung und Einbürgerung in enger Verbindung zur Sozialintegration stehen. Die in Kap. 3 referierten Forschungsergebnisse deuten auf einen solchen Zusammenhang hin.

Aber worin besteht die eigentliche Substanz dieser Bindungen? Mit der oben dargestellten sozialen Produktionsfunktion steht ein Konstrukt zur Verfügung, das es ermöglicht, den Bogen von Handlungsentscheidung zum Bindungsgedanken zu schlagen. Die Elemente Kulturation, Platzierung und Interaktion erschließen die Möglichkeiten in einer Gesellschaft die eigenen Ziele zu verwirklichen. Identifikation kann hierbei als affektuelle und unterstützende Komponente angesehen werden. Bindung ist demnach nur ein anderer Begriff für die Zugänglichkeit einer bestehenden Gelegenheitsstruktur zur Erlangung von physischem Wohlbefinden und sozialer Wertschätzung. Ausgehend von RREEMM-Modell rationaler Entscheidung sollte der Entschluss zur Abwanderung bzw. Einbürgerung auf dieses Grundmotiv menschlicher Handlung zurückzuführen sein. Problematisch an dieser Perspektive ist die Komplexität der Vorgänge, die nicht ohne weiteres eine individuelle Handlung repräsentiert, sondern oft das Resultat interdependenter Handlungen mehrerer Akteure ist. Allerdings folgen nicht nur die Immigranten dem Kalkül der sozialen Produktionsfunktionen, sondern auch die soziale Umwelt erkennt die bestehenden Bindungen in der Regel an. ${ }^{38}$

38 Entscheidungen staatlicher Instanzen - z.B. in der Bundesrepublik zum Aufenthaltsrecht - orientieren sich u.a. an der Aufenthaltsdauer, den familiären Beziehungen, am Erwerbstatus und den sprachlichen Kompetenzen (vgl. Bischoff/Teubner 1991, 68-77) 
Welche Kausalstruktur sich hinter diesen Zusammenhängen verbirgt, ist eine Frage, der im empirischen Teil nachgegangen werden soll. Als Ausgangspunkt dient hierfür der auf das Integrationsniveau hin interpretierte Kern des von Esser entworfenen Prozessmodells (vgl. Abb. 4.1.1a). ${ }^{39}$ In dieses Modell wird zusätzlich lediglich noch eine endogene Variable eingefügt, die sich aus dem Auftreten bzw. Ausbleiben von Abwanderung und Einbürgerung ergibt (vgl. Abb 4.4.3a).

\section{Abbildung 4.4.3a: Erklärungsmodell zur Einbürgerung und Abwanderung basie- rend auf dem Kern des Prozessmodells von 1980}

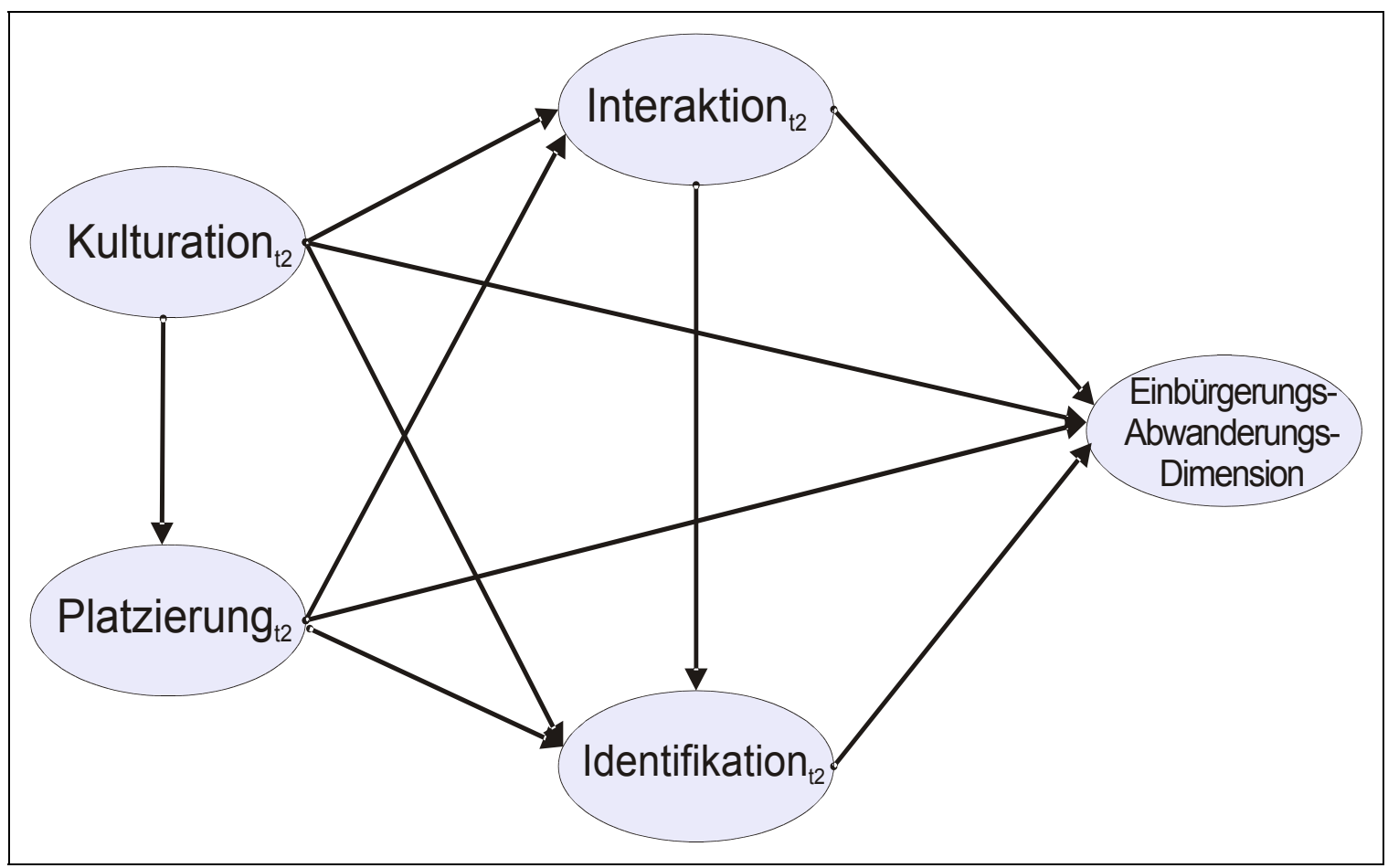

Ob sich tatsächlich alle Subdimensionen der Sozialintegration als erklärungskräftig erweisen werden ist ungewiss, zumal die kausalen Beziehungen der Subdimen-

39 Im Folgenden wird auch im Rahmen von individuellen Prozessen der Terminus der Sozialintegration bevorzugt, dementsprechend werden in den skizzierten Modellen die Bezeichnungen der Teildimensionen der Assimilation mit denen der Sozialintegration ersetzt (kognitive Assimilation = Kulturation; strukturelle Assimilation = Platzierung; soziale Assimilation = Interaktion; identifikative Assimilation = Identifikation). 
sionen untereinander bislang in der vorliegenden Form nicht geprüft wurden. ${ }^{40}$ Das vorliegende Modell muss tendenziell als heuristisch verstanden werden, um in der empirischen Prüfung eingehender zu klären, welche der einzelnen Aspekte der Sozialintegration als wesentlich in Hinsicht auf die Bindung an die Aufnahme- bzw. Mehrheitsgesellschaft zu betrachten sind. Werden alle potentiell möglichen kausalen Beziehungen zwischen Erklärungsmodell und zu erklärender Variable berücksichtigt, dann lassen sich hieraus einige Hypothesen ableiten. So sollte es umso eher zu Einbürgerung und umso weniger zur Abwanderung kommen, je intensiver sich Immigranten ak- oder ihre Nachkommen enkulturieren. Je höher die strukturelle Assimilierung in Form der Platzierung auf bevorzugten Positionen in der Gesellschaft ausfällt, desto wahrscheinlicher sollte Einbürgerung und umso unwahrscheinlicher sollte Abwanderung werden. Es sollte umso mehr Einbürgerungsvorgänge und umso seltener Abwanderungen geben, je mehr soziale Beziehungen von Personen mit Migrationshintergrund zu Mitgliedern der autochthonen Bevölkerung unterhalten werden. Und nicht zuletzt, je enger die emotionale Bindung ausgedrückt über Identifikation mit der Aufnahme- bzw. Mehrheitsgesellschaft empfunden wird, desto häufiger sollte es zu Einbürgerungen und entsprechend weniger zu Abwanderungen kommen.

In einem zweiten Schritt lässt sich darauf aufbauend der Sozialintegrationsprozess in seiner Wirkung auf die Erklärung der Einbürgerungs- und Abwanderungsvorgänge betrachten. Die Hypothesen können, bedingt durch die schrittweise Erweiterung, auch auf den gesamten Prozess übertragen werden. Für die geplante Erklärung der Einbürgerungs- und Abwanderungsvorgänge auf Basis der Sozialintegration wäre die reduzierte Modellierung des Prozessmodells hinreichend, zumal die ausgeschlossenen Konstrukte (Merkmale der Person, Umgebung und Aufenthaltsdauer) mehr der Erklärung der Sozialintegration dienen als der Erklärung der weiteren Einbürgerung und Abwanderung.

40 Ein sehr ähnliches Modell konnte Esser 1981 mittels Pfadanalyse an den Daten einer Erhebung unter Arbeitsmigranten in der Bundesrepublik bestätigen (Esser 1981). Auf Grundlage eines etwas modifizierten Modells gelangte Paul Hill 1984 zu einem ähnlichen Ergebnis (vgl. Hill 1984, 176-182). 


\section{Abbildung 4.4.3b: Erklärungsmodell zur Einbürgerung und Abwanderung basie- rend auf dem Prozess der Sozialintegration}

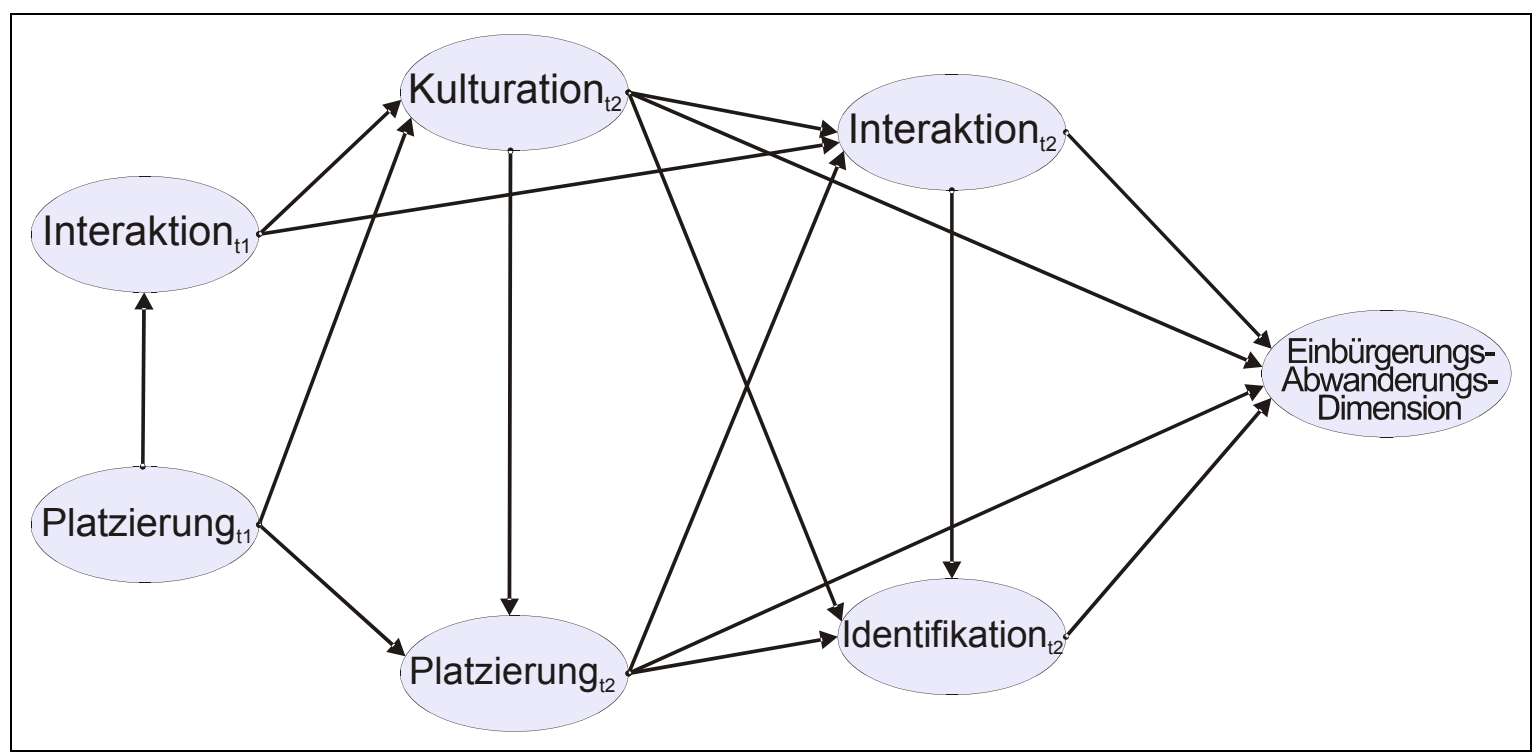

Insofern würde erwartet (vgl. Abb. 4.3.3b), dass die erreichten Niveaus zum dritten Zeitpunkt $\left(\mathrm{t}_{2}\right)$ im Bereich der Kulturation, Platzierung, Interaktion und Identifikation jeweils einen Effekt auf die festzustellenden Einbürgerungs- und Abwanderungsvorgänge haben. Die Interaktion und Platzierung in der ersten Phase des Aufenthaltes sollten über diese indirekt auf die zu erklärenden Vorgänge wirken. Von der Konzeption und den geäußerten Erwartungen her betrachtet, sollten in beiden Fällen die Platzierung und die damit eng verbundene Kulturation eine wesentlich Rolle im Erklärungsmodell spielen, da Esser insbesondere die Platzierung als „Schlüssel für jede nachhaltige Sozialintegration“ bezeichnet (Esser 2001, 10).

\subsection{Zusammenfassung und Diskussion}

Das von Esser vorgeschlagene Modell der Sozialintegration, welches explizit in der Tradition der Assimilationsforschung steht, wurde in der hier referierten Form als ergebnisoffener Assimilation-Dissimilations-Ansatz interpretiert. Im Zusammenhang mit den Entscheidungen über Handlungsalternativen steht es den Akteuren insofern frei, sich jederzeit, ausgehend von ihrem jeweiligen Niveau des Intergriert-Seins, für 
oder gegen weitere assimilative bzw. dissimilative Handlungen zu entscheiden. Um genauer zwischen Prozessen und Zuständen unterscheiden zu können, werden in der vorliegenden Arbeit, anders als bei Esser, Integration und Assimilation allgemein als Prozesse definiert, die Zustände des Integriert- bzw. Assimiliert-Seins nach sich ziehen (vgl. Esser 2001, 1, ebd. 19-20).

Aufbauend auf der analytischen Trennung von System- und Sozialintegration wird das Konzept der Sozialintegration untergliedert in vier Subdimensionen als Grundmodell der empirischen Analyse dienen. Dazu werden die Teildimensionen des von Esser vorgeschlagenen Kausal-Modells als Prädiktoren der Abwanderung und Einbürgerung verwendet (vgl. Abb. 4.4.3a).

Bezugspunkt des Modells ist dabei das vollständige Integriert-Sein über Assimilation bezogen auf alle Teildimensionen. Als alternative Entwicklungstendenzen werden entlang der Integrationsdimensionen bezogen auf die Aufnahmegesellschaft und die ethnische Gemeinschaft Marginalität, Segmentation und Mehrfachintegration diskutiert. Diese Idealtypen des Sozialintegrationszustands sind als Pole zu verstehen, zwischen denen sich die Anpassungsprozesse bewegen. Für das Verständnis der realen Integrationsergebnisse ist es deshalb hilfreich, auf die Idee der hybriden Identität zurückzugreifen, denn im Gegensatz zu Esser, der Identität im Sinne eines „einfachen binären Musters“ strukturiert sieht, gibt es Hinweise, dass hybride Muster zwischen Assimilation und Segmentation keine Seltenheit sind (ebd., 21). Diese Hybridität findet z.B. ihren sprachlichen Ausdruck in Bindestrichbezeichnungen wie Deutsch-Türken, der teilweise von den in Deutschland lebenden Personen türkischer Herkunft als Eigenbezeichnung bevorzugt wird (vgl. Sackmann/Schulz 2001, 42).

Das Modell der Sozialintegration und auch das Prozessmodell der Assimilation basieren insgesamt auf einer am SEU-Ansatz orientierten Handlungstheorie, die über das Konzept der sozialen Produktionsfunktionen eine Erklärung für die unterschiedlichen Integrationsverläufe der Personen mit Migrationshintergrund liefert. Als grundlegende Motive menschlicher Handlungen werden im Anschluss an Lindenberg physisches Wohlbefinden und soziale Anerkennung postuliert. 
Im Verlauf der Sozialintegration eines Immigranten oder seiner Nachkommen führen Entscheidungen für assimilative Handlungen zu einer weitergehenden Einbindung in die Mehrheitsgesellschaft, während Dissimilation die Verbindungen zu ethnischen Kolonien und transnationalen Bezugspunkten fördert. Einbürgerung und Abwanderung können dementsprechend als Resultate der Sozialintegration angesehen werden. Die beiden betrachteten Vorgänge können ihrerseits wieder Rückwirkungen im Hinblick auf die Zusammensetzung der ethnischen Gruppen und damit auf das Konfliktpotential zwischen den ethnischen Gruppen besitzen. 


\section{Daten und Methoden}

Nach dem nun im theoretischen Teil dieser Arbeit die Verbindung der Sozialintegration mit den Einbürgerungs- und Abwanderungsvorgängen auf Grundlage des Ansatzes von Esser vorgestellt worden ist, sollen die theoretisch begründeten Kausalbeziehungen der einzelnen Konstrukte anhand empirischer Daten überprüft werden. Mit dem Vorhaben der Überprüfung des vorgestellten Kausalmodells sind einige empirische Konsequenzen verbunden, die es nicht leicht machen, einen entsprechenden Datensatz zu finden, denn nicht jede Erhebung unter Immigranten kann die notwendigen Voraussetzungen erfüllen.

Eine der wesentlichen Grundbedingungen, denen die Daten entsprechen müssen, ist, die erforderlichen Variablen zu unterschiedlichen Zeitpunkten oder aber retrospektiv von Immigranten und gegebenenfalls von deren Nachkommen zu erheben. Hinsichtlich der Datenstruktur ist dabei dem Panelansatz der Vorzug zu geben, da die Erinnerung, insbesondere emotionaler Identifikation nach einem einschneidenden Ereignis, wie es eine Einbürgerung und auch eine Abwanderung darstellen, Veränderungen und Rekonstruktionen unterworfen sein kann (vgl. Reimer 2001, 4446). Zudem müssten im Falle der Retrospektiverhebung nicht nur im Einbürgerungsland, sondern auch an den Zielorten der Abwanderung Befragungen durchgeführt werden, was den erhebungstechnischen Aufwand unverhältnismäßig erhöhen würde; zumal kein Register geführt wird, das die Zielrichtungen der Abwanderungen ehemaliger Immigranten systematisch erfasst.

Eine weitere Voraussetzung, die zwingend für den geplanten Modelltest gegeben sein müsste, ist das Vorliegen von Operationalisierungen der einzelnen Komponenten des Modells. Das Erhebungsinstrument müsste Fragen zur Aufenthaltsdauer, zur Erwerbsbiographie, zur sozialen Stellung, zur sprachlich-kulturellen Anpassung, zum Kontakt mit der autochthonen Bevölkerung, zur Identifikation mit der Aufnahmegesellschaft sowie die Informationen über Abwanderungs- und Einbürgerungsvorgänge beinhalten, die kontinuierlich oder zumindest in der richtigen Reihenfolge im Fragenprogramm eines Panels erhoben worden sein müssten. 
In der Bundesrepublik Deutschland existiert meiner Kenntnis zufolge nur ein Datensatz, der die notwendigen Anforderungen erfüllen könnte. Es handelt sich dabei um das Sozio-oekonomische Panel (SOEP) des Deutschen Instituts für Wirtschaftsforschung (DIW), das im folgenden Abschnitt kurz vorgestellt und bezogen auf seine Verwendbarkeit hin untersucht werden soll. Im Anschluß daran steht die Erörterung der Konsequenzen, die sich aus der Datenlage für die beabsichtigte Untersuchung ergeben, um dann auf die Frage einzugehen, welches statistische Analysemodell unter den gegebenen Umständen als geeignet angesehen werden kann.

\subsection{Das Sozio-oekonomische Panel des Deutschen Insti- tuts für Wirtschaftsforschung: Konzeption und Design}

Seit nunmehr zweiundzwanzig Jahren ist das Sozio-oekonomische Panel neben den Daten des Statistischen Bundesamtes und der Allgemeinen Bevölkerungsumfrage der Sozialwissenschaften die umfangreichste sozialwissenschaftliche Datenquelle in der Bundesrepublik. Von seinem Forschungsdesign her betrachtet geht das SOEP über den klassischen Längschnittansatz hinaus und verbindet diesen „mit der retrospektiven Erhebung von Ereignisdaten“ (Blossfeld/Hamerle/Mayer 1986). Die gewählte Herangehensweise ermöglicht es, Stabilität und Veränderung ins Verhältnis zu wichtigen Ereignissen im Leben des Befragten zu setzen. Mit diesem erweiterten Paneldesign startete das SOEP 1984 mit der ersten Welle angelegt als Haushaltspanel in zwei separaten Stichproben. Stichprobe A sollte 4.500 und die Stichprobe B 1.500 Privathaushalte ${ }^{41}$ umfassen, tatsächlich wurden 4.528 und 1.393 Haushalte 1984 erreicht. Stichprobe A wird in der Regel als „deutsche Stichprobe“ bezeichnet, obwohl die Zuordnung etwas anders erfolgte: Sofern der Haushaltsvorstand nicht die Staatsangehörigkeit einer der fünf großen Gastarbeiternationen (griechisch, italienisch, jugoslawisch, spanisch und türkische Nationalität) besaß, wurde der Haushalt der Stichprobe A zugeordnet, andernfalls der Stichprobe B. Was dazu führte, dass auch in Stichprobe A einige Immigranten vorhanden sind (Haisken-DeNew/Frick 2003, 18).

41 In dem Auswahlprozess blieben Personen unberücksichtigt, die zur Zeit der ersten Welle in Gemeinschaftsunterkünften untergebracht waren. Sofern Personen aus den SOEP-Haushalten in späteren Jahren in Gemeinschaftsunterkünfte umzogen, wurde dies weiterhin für das Panel berücksichtigt. 
In den darauf folgenden Jahren sind bis 2002 fünf weitere Stichproben aus unterschiedlichen Gründen zu den ersten beiden hinzugekommen. Bedingt durch die Vereinigung von DDR und BRD wurde 1991 eine neue Stichprobe von 2.179 ostdeutschen Privathaushalten in das SOEP aufgenommen. In Folge der erhöhten Einwanderung nach Westdeutschland in den frühen 1990er Jahren wurden 1994 und 1995 die separaten Stichproben D1 $(n=236)$ und D2 $(n=295)$ gestartet, die zusammen 522 Haushalte umfassten, in denen zumindest eine Person lebte, die nach 1984 in die alten Bundesländer zugewandert sein musste. In den Jahren 1998 und 2000 wurden basierend auf dem Auswahlverfahren der Stichprobe A neue unabhängige Stichproben ( $E$ und $F$ ) gezogen, die zusammen rund 7.119 Haushalte zusätzlich dem SOEP zuführten. Als letzte Erweiterung wurde ebenfalls unabhängig von den vorhergehenden Stichproben 2002 die Stichprobe G für private Haushalte mit einem monatlichen Haushaltseinkommen von mindestens $3.835 €$ (7.500 DM) in das Panelprogramm aufgenommen. Die Anzahl der dadurch neu hinzugekommenen Haushalte betrug im ersten Jahr 1.224 Haushalte. Im Zuge dieser Erweiterungen hatte sich der Stichprobenumfang im Jahr 2000 auf einen Maximalwert von 13.266 Haushalten erhöht, was einer Gesamtzahl von 24.597 Personen im Panel entsprach (vgl. Haisken-DeNew/Frick 2003, 17-19).

\subsubsection{Teilnehmer des Sozio-oekonomischen Panels mit Migrationshintergrund}

Als originäre Gastarbeiterstichprobe wurden nur die Stichproben B angelegt. Bedingt durch die in den anderen Fällen verwendeten Auswahlregeln und Einzüge in SOEP-Haushalte sind auch in anderen Teilstichproben Immigranten vorhanden, wobei nicht davon ausgegangen werden kann, dass auch alle als Immigranten im Datensatz zu erkennen sind. Aber auch die Zugehörigkeit zu einem Haushalt mit ausländischem Haushaltsvorstand ist nicht immer eindeutig, da binationale Ehen und Partnerschaften keine Seltenheit darstellen.

Orientiert an der Staatsangehörigkeit waren im Jahr 1984 3.198 Personen mit einer anderen als der deutschen Staatsbürgerschaft Angehörige eines Haushalts der 
Tabelle 5.1.1a: Anzahl der Personen mit einer ausländischen Staatsangehörigkeit aufgeschlüsselt nach Jahr der Erhebung und den einzelnen SOEP-Stichproben

\begin{tabular}{|c|c|c|c|c|c|c|c|c|}
\hline & \multicolumn{7}{|c|}{ SOEP-Stichproben } & \multirow[b]{2}{*}{ Summe } \\
\hline Jahr & A & B & C & D & $\mathbf{E}$ & $\mathbf{F}$ & $\mathbf{G}$ & \\
\hline 1984 & 149 & 3.049 & - & - & - & - & - & 3.198 \\
\hline 1985 & 130 & 2.601 & - & - & - & - & - & 2.731 \\
\hline 1986 & 123 & 2.500 & - & - & - & - & - & 2.623 \\
\hline 1987 & 113 & 2.497 & - & - & - & - & - & 2.610 \\
\hline 1988 & 104 & 2.400 & - & - & - & - & - & 2.504 \\
\hline 1989 & 95 & 2.354 & - & - & - & - & - & 2.449 \\
\hline 1990 & 97 & 2.318 & - & - & - & - & - & 2.415 \\
\hline 1991 & 94 & 2.322 & 15 & - & - & - & - & 2.431 \\
\hline 1992 & 87 & 2.286 & 11 & - & - & - & - & 2.384 \\
\hline 1993 & 88 & 2.251 & 9 & - & - & - & - & 2.348 \\
\hline 1994 & 79 & 2.144 & 10 & 32 & - & - & - & 2.265 \\
\hline 1995 & 82 & 2.002 & 11 & 236 & - & - & - & 2.331 \\
\hline 1996 & 90 & 1.877 & 12 & 191 & - & - & - & 2.170 \\
\hline 1997 & 96 & 1.790 & 11 & 154 & - & - & - & 2.051 \\
\hline 1998 & 87 & 1.640 & 8 & 116 & 75 & - & - & 1.926 \\
\hline 1999 & 80 & 1.520 & 9 & 98 & 57 & - & - & 1.764 \\
\hline 2000 & 83 & 1.410 & 9 & 99 & 52 & 701 & - & 2.354 \\
\hline 2001 & 78 & 1.343 & 7 & 86 & 50 & 499 & - & 2.063 \\
\hline 2002 & 79 & 1.224 & 7 & 78 & 48 & 415 & 70 & 1.921 \\
\hline 2003 & 84 & 1.127 & 8 & 73 & 45 & 367 & 54 & 1.758 \\
\hline 2004 & 81 & 1.052 & 9 & 67 & 43 & 340 & 50 & 1.642 \\
\hline
\end{tabular}

Quelle: SOEP Wellen 1-21

Stichproben A und B (vgl. Tab. 5.1.1a). Bedingt durch die üblichen Panelausfälle und durch Einbürgerungen sank diese Zahl über alle Stichproben hinweg auf 1.642 in 2004, obwohl durch zusätzliche Stichproben dieser Prozess etwas verzögert werden konnte (ausführlich hierzu Schräpler 2000). Insbesondere die Stichproben D2 und $E$ trugen zu einer Vergrößerung der Ausländerstichprobe bei. Der Anteil der Stichprobe B am Gesamtaufkommen an Personen mit einer ausländischen Staatsangehörigkeit sank dementsprechend von über 95\% 1984 auf rund 64\% im Jahr 
2004. Von diesen Zahlen ausgehend ist der Anteil an Immigranten und ihren Nachkommen nur grob einzuschätzen, da Personen, die unter Aufgabe ihrer ursprünglichen Staatsangehörigkeit bereits eingebürgert waren, hierin ebenso wie Aussiedler nur zum Teil enthalten sind.

Etwas genauere Informationen dahingehend, wer als Immigrant zu zählen ist, enthält eine weitere Variable, die darüber Auskunft gibt, ob eine Person in Deutschland geboren bzw. vor 1949 immigrierte oder nach 1948 zugewandert ist. Die Zahl von 7.052 Zuwanderern enthält damit neben den klassischen Gastarbeitern und Einwanderern ohne ethnischen Bezug zu Deutschland allerdings auch Aussiedler und Kinder deutscher Eltern, die im Ausland geboren wurden (vgl. Tab. 5.1.1b).

Tabelle 5.1.1b: Anzahl der Personen im SOEP, die nach 1948 in die Bundesrepublik bzw. die Deutsche Demokratische Republik zuwanderten

\begin{tabular}{|l|r|r|}
\hline \multicolumn{2}{|c|}{ Häufigkeiten } & \multicolumn{1}{|c|}{ Prozent } \\
\hline in Deutschland geb. & 36.382 & 80,6 \\
\hline oder immigr. vor 1949 & $\mathbf{7 . 0 5 2}$ & $\mathbf{1 5 , 6}$ \\
\hline immigr. nach 1948 & 1.710 & 3,8 \\
\hline fehlende Werte & 45.144 & 100,0 \\
\hline Gesamt & \\
\hline
\end{tabular}

Quelle: SOEP Wellen 1-21

Werden die Informationen zur Immigration und Staatsbürgerschaft miteinander verknüpft, dann lässt sich relativ eindeutig eine Immigrantengruppe bestimmen, die im Sinne des zu testenden Modells klassische Einwanderer darstellen und die im Unterschied zu Aussiedlern nicht sofort bei Einreise in die Bundesrepublik Deutschland als Staatsbürger anerkannt werden. Während sich zu Beginn des Sozio-oekonomischen Panels 2.876 Immigranten unter den Personen mit ausländischer Staatsangehörigkeit befanden, waren es 2004 nur noch 1.394. Der Rückgang erfolgte bis 1999 kontinuierlich, bedingt durch die höhere Panelmortalität der Immigranten gegenüber den in Deutschland geborenen Personen mit ausländischer Staatsangehörigkeit. 
Auffallend ist, dass im Jahr 2000 im Rahmen der Auffrischung durch die Stichprobe F die Zahl an Ausländern insgesamt erhöht werden konnte, die bedingt durch höhere Ausfälle unter den in Deutschland geborenen Personen mit ausländischer Staatsangehörigkeit im Jahr 2001 kurzfristig zu einer Erhöhung des Anteils führte. Mit dem Jahr 2002 begann der Anteil an Immigranten wieder zu sinken und lag im Jahr 2003 bei rund 71,7 \% (vgl. Tab. 5.1.1c).

Tabelle 5.1.1c: Anzahl der Immigranten im Vergleich zu Personen ohne deutsche Staatsangehörigkeit im SOEP nach Erhebungsjahr

\begin{tabular}{||r|r|r|r|}
\hline Jahr & Ausländer & $\begin{array}{c}\text { davon } \\
\text { Immigranten }\end{array}$ & Prozentanteil \\
\hline 1984 & 3.212 & $\mathbf{2 . 8 7 6}$ & 89,54 \\
\hline 1985 & 2.745 & $\mathbf{2 . 4 2 5}$ & 88,34 \\
\hline 1986 & 2.647 & $\mathbf{2 . 3 2 0}$ & 87,65 \\
\hline 1987 & 2.642 & $\mathbf{2 . 3 0 1}$ & 87,09 \\
\hline 1988 & 2.536 & $\mathbf{2 . 1 7 1}$ & 85,61 \\
\hline 1989 & 2.481 & $\mathbf{2 . 0 9 1}$ & 84,28 \\
\hline 1990 & 2.453 & $\mathbf{2 . 0 3 3}$ & 82,88 \\
\hline 1991 & 2.458 & $\mathbf{1 . 9 8 6}$ & 80,80 \\
\hline 1992 & 2.441 & $\mathbf{1 . 9 3 7}$ & 79,35 \\
\hline 1993 & 2.416 & $\mathbf{1 . 8 9 4}$ & 78,39 \\
\hline 1994 & 2.340 & $\mathbf{1 . 8 1 4}$ & 77,52 \\
\hline 1995 & 2.419 & $\mathbf{1 . 9 1 2}$ & 79,04 \\
\hline 1996 & 2.291 & $\mathbf{1 . 7 7 7}$ & 77,56 \\
\hline 1997 & 2.195 & $\mathbf{1 . 6 7 0}$ & 76,08 \\
\hline 1998 & 2.092 & $\mathbf{1 . 5 5 2}$ & 74,19 \\
\hline 1999 & 1.943 & $\mathbf{1 . 4 3 8}$ & 74,01 \\
\hline 2000 & 2.575 & $\mathbf{1 . 7 9 5}$ & 69,71 \\
\hline 2001 & 2.314 & $\mathbf{1 . 7 6 2}$ & 76,15 \\
\hline 2002 & 2.184 & $\mathbf{1 . 6 0 6}$ & 73,53 \\
\hline 2003 & 2.031 & $\mathbf{1 . 4 7 5}$ & 72,62 \\
\hline 2004 & 1.943 & $\mathbf{1 . 3 9 4}$ & 71,74 \\
\hline
\end{tabular}

Quelle: SOEP Wellen 1-21 


\subsubsection{Das Fragenprogramm des SOEP}

Angelegt als Haushaltspanel mit expliziter Berücksichtigung von Ereignisdaten stützt sich das SOEP als Haupterhebungsmethode auf von Interviewern im direkten Gespräch ausgefüllte PAPI-Fragebögen, seit 1998 werden in zunehmendem Maße auch Notebooks für computerunterstützte Erhebungen genutzt. Über die Jahre sind neben klassischen Face-to-face-Interviews zudem vom Respondenten selbst auszufüllende Fragebögen zunächst auf Papier, später per E-Mail und via Online-Fragebögen (http-Protokoll) zum Einsatz gekommen. Eine eher untergeordnete Rolle spielen CATI- und Proxy-Interviews.

Tabelle 5.1.2a: Übersicht zur thematischen Ausrichtung der Panelwellen des Sozio-oekonomischen Panels

\begin{tabular}{|c|c|c|c|c|c|c|c|c|c|c|c|c|}
\hline & \multicolumn{12}{|c|}{ Themen } \\
\hline Jahr & $\begin{array}{c}\text { Erwerbs- } \\
\text { biogra- } \\
\text { phie }\end{array}$ & $\begin{array}{l}\text { Ehe- und } \\
\text { Familien- } \\
\text { biogra. }\end{array}$ & $\begin{array}{l}\text { Soziale } \\
\text { Herkunft } \\
\text { Berufs- } \\
\text { einstieg }\end{array}$ & $\begin{array}{c}\text { Wohnum- } \\
\text { feld }\end{array}$ & $\begin{array}{l}\text { Soziale } \\
\text { Siche- } \\
\text { rung }\end{array}$ & $\begin{array}{c}\text { Vermö- } \\
\text { gens- } \\
\text { bilanz }\end{array}$ & \begin{tabular}{|c|} 
berufl. \\
Qualifi- \\
zierung
\end{tabular} & \begin{tabular}{|c|} 
Zeitver- \\
wendung \\
und -prä- \\
ferenzen
\end{tabular} & \begin{tabular}{|c|} 
Familie \\
und \\
soziale \\
Aktivitäten
\end{tabular} & $\begin{array}{c}\text { Arbeits- } \\
\text { beding- } \\
\text { ungen }\end{array}$ & $\begin{array}{c}\text { Zukunfts- } \\
\text { erwart. }\end{array}$ & $\begin{array}{l}\text { Umwelt- } \\
\text { verhalt. }\end{array}$ \\
\hline 1984 & $x$ & & & & & & & & & & & \\
\hline 1985 & $x$ & $x$ & & & & & & & & $x$ & & \\
\hline 1986 & $x$ & $x$ & $x$ & $x$ & & & & & & $x$ & & \\
\hline 1987 & $x$ & $x$ & $x$ & & $x$ & & & & & $x$ & & \\
\hline 1988 & $x$ & $x$ & $x$ & & & $x$ & & & & & & \\
\hline 1989 & $x$ & $x$ & $x$ & & & & $x$ & & & & & \\
\hline 1990 & $x$ & $x$ & $x$ & & & & & $x$ & & & & \\
\hline 1991 & $x$ & $x$ & $x$ & & & & & & $x$ & & & \\
\hline 1992 & $x$ & $x$ & $x$ & & $x$ & & & & & & & \\
\hline 1993 & $x$ & $x$ & $x$ & & & & $x$ & & & & & \\
\hline 1994 & $x$ & $x$ & $x$ & $x$ & & & & & & & $x$ & \\
\hline 1995 & $x$ & $x$ & $x$ & & & & & $x$ & & $x$ & & \\
\hline 1996 & $x$ & $x$ & $x$ & & & & & & $x$ & & & \\
\hline 1997 & $x$ & $x$ & $x$ & & $x$ & & & & & & & \\
\hline 1998 & $x$ & $x$ & $x$ & & & & & & & & & $x$ \\
\hline 1999 & $x$ & $x$ & $x$ & $x$ & & & & & & & $x$ & \\
\hline 2000 & $x$ & $x$ & $x$ & & & & $x$ & $x$ & & & & \\
\hline 2001 & $x$ & $x$ & $x$ & & & $x$ & & & $x$ & $x$ & & \\
\hline 2002 & $x$ & $x$ & $x$ & & $x$ & $x$ & & & & & & \\
\hline 2003 & $x$ & $x$ & $x$ & & & $x$ & & & $x$ & $x$ & & $x$ \\
\hline 2004 & $x$ & $x$ & $x$ & & & & & & & & $x$ & \\
\hline
\end{tabular}

Quelle: SOEP Wellen 1-21 
Mittels dieses breiten Spektrums an Erhebungsverfahren ist im Laufe der Jahrzehnte ein enormes Fragenprogramm zum Teil mehrfach abgearbeitet worden. Aber nicht alle Fragen wurden, mit Rücksicht auf die Dauer der Interviews, in jeder der jährlichen Welle erhoben. Jährlich wurden bisher die Erwerbs- und Familienbiographie sowie die soziale Herkunft erhoben bzw. nach Änderungen gefragt. Themen wie soziale Sicherung, Wohnumfeld, Vermögen, berufliche Qualifikation, Familie- und soziale Aktivitäten, Zeitverwendung, Arbeitsbedingungen, Zukunftserwartungen und Umweltverhalten wurden nicht parallel, sondern meist in einem asynchronen Fünfjahres-Rhythmus wiederholt (vgl. Tab. 5.1.2a).

Für ausländische Befragte gab es ein zusätzliches Fragenprogramm, das 1996 den veränderten Rahmenbedingungen angepasst worden ist. ${ }^{42}$ Die für die weiteren Analysen wichtigsten ausländerspezifischen Themenbereiche sind Aufenthaltsabsichten, Sprache, ethnische Identifikation, soziale Kontakte und ökonomische Aspekte, wie Überweisungen an Verwandte im Ausland und Investitionen im Herkunftsland. Wie aus Tabelle 5.1.2b hervorgeht, wurden die genannten Themenkomplexe entweder jährlich oder jedes zweite Jahr erhoben. Des Weiteren sind die Informationen zur Aufenthaltsdauer nur bis 1993 erhoben worden, was aber bedingt durch die Beschränkung auf den assimilativen Kern nicht weiter ins Gewicht fällt. Für die nach 1993 hinzugekommenen ausländischen Befragten liegen nur vage Anhaltspunkte in Form der Fragen nach dem Geburtsland und ob die Befragten bereits 1984 in Deutschland gelebt haben vor. Die Angaben zur Abwanderung und Staatsangehörigkeit sind durchgängig erfasst worden, hingegen wird der Besitz der deutschen Staatsangehörigkeit explizit erst seit 1996 erhoben. Das Jahr der Einbürgerung wurde nur einmalig retrospektiv in 2002 erfragt.

Über die genannten Sachverhalte hinaus bleibt zu den ausländerspezifischen Erhebungen noch festzuhalten, dass nur für Immigranten aus klassischen Gastarbeiterländern die Möglichkeit gegeben ist, zwischen Deutsch und der Hauptverkehrssprache des Heimatlandes als Befragungssprache zu wählen.

42 Beispielsweise wurde die Frage zu den Rückkehrabsichten gegen die Frage, ob der Befragte für immer in Deutschland bleiben wolle, ausgetauscht. 
Tabelle 5.1.2b: Übersicht zu ausgewählten ausländerspezifischen Themenkomplexen im Sozio-oekonomischen Panel

\begin{tabular}{|c|c|c|c|c|c|c|c|c|c|c|c|c|}
\hline \multirow[b]{2}{*}{ Jahr } & \multicolumn{12}{|c|}{ Themen } \\
\hline & $\begin{array}{c}\text { Aufent- } \\
\text { haltsab- } \\
\text { sichten }\end{array}$ & Sprache & $\begin{array}{l}\text { ethnische } \\
\text { Identifi- } \\
\text { kation }\end{array}$ & $\begin{array}{c}\text { Soziale } \\
\text { Kontakte }\end{array}$ & $\begin{array}{l}\text { ökonom. } \\
\text { Aspekte }\end{array}$ & $\begin{array}{c}\text { Jahr der } \\
\text { Immi- } \\
\text { gration }\end{array}$ & \begin{tabular}{|c|}
1984 in \\
Deutsch- \\
land
\end{tabular} & $\begin{array}{l}\text { in } \\
\text { Deutsch- } \\
\text { land } \\
\text { geboren }\end{array}$ & $\begin{array}{l}\text { Abwan- } \\
\text { derung }\end{array}$ & $\begin{array}{c}\text { Staatsan- } \\
\text { gehörig- } \\
\text { keit }\end{array}$ & $\begin{array}{l}\text { Jahr der } \\
\text { Einbür- } \\
\text { gerung }\end{array}$ & $\begin{array}{c}\text { Deutsche } \\
\text { Staatsan- } \\
\text { gehörigkeit } \\
\text { (ja/nein) }\end{array}$ \\
\hline 1984 & $x$ & $x$ & $x$ & & $x$ & $x$ & & & & $x$ & & \\
\hline 1985 & $x$ & $x$ & $x$ & $x$ & $x$ & $x$ & & & $x$ & $x$ & & \\
\hline 1986 & $x$ & $x$ & $x$ & $x$ & $x$ & $x$ & & & $x$ & $x$ & & \\
\hline 1987 & $x$ & $x$ & $x$ & $x$ & $x$ & $x$ & & & $x$ & $x$ & & \\
\hline 1988 & $x$ & & & & $x$ & $x$ & & & $x$ & $x$ & & \\
\hline 1989 & $x$ & $x$ & $x$ & $x$ & $x$ & $x$ & & & $x$ & $x$ & & \\
\hline 1990 & $X^{*}$ & & & & $X^{*}$ & $X^{*}$ & & & $x$ & $x$ & & \\
\hline 1991 & $\mathbf{X}^{*}$ & $X^{*}$ & $X^{*}$ & $X^{*}$ & $\mathbf{X}^{*}$ & $X^{*}$ & & & $x$ & $x$ & & \\
\hline 1992 & $x$ & & & & & $x$ & & & $x$ & $x$ & & \\
\hline 1993 & $x$ & $x$ & $x$ & $x$ & $x$ & $x$ & & & $x$ & $x$ & & \\
\hline 1994 & $x$ & & & & & & & & $x$ & $x$ & & \\
\hline 1995 & $x$ & $x$ & $x$ & $x$ & $x$ & & $x$ & & $x$ & $x$ & & \\
\hline 1996 & $x$ & & & & $x$ & & $x$ & & $x$ & $x$ & & $x$ \\
\hline 1997 & $x$ & $x$ & $x$ & $x$ & $x$ & & $x$ & $x$ & $x$ & $x$ & & $x$ \\
\hline 1998 & $x$ & & & & $x$ & & $x$ & $x$ & $x$ & $x$ & & $x$ \\
\hline 1999 & $x$ & $x$ & $x$ & $x$ & $x$ & & $x$ & $x$ & $x$ & $x$ & & $x$ \\
\hline 2000 & $x$ & & & & $x$ & & $x$ & $x$ & $x$ & $x$ & & $x$ \\
\hline 2001 & $x$ & $x$ & $x$ & $x$ & $x$ & & $x$ & $x$ & $x$ & $x$ & & $x$ \\
\hline 2002 & $x$ & & & & $x$ & & & $x$ & $x$ & $x$ & $x$ & $x$ \\
\hline 2003 & $x$ & $x$ & $x$ & $x$ & $x$ & & & $x$ & $x$ & $x$ & & $x$ \\
\hline 2004 & $x$ & & & & $x$ & & & $x$ & $x$ & $x$ & & $x$ \\
\hline
\end{tabular}

Quelle: SOEP Wellen 1-21

\subsubsection{Operationalisierung der Konzepte des Kausalmodells}

Es bleibt nun zu klären, ob in den Erhebungsinstrumenten der einzelnen Wellen verwendbare Indikatoren zu finden sind, ob diese gegebenenfalls die richtige zeitliche Abfolge aufweisen und ob es zudem relevante Änderungen gab, die im Rahmen der geplanten Analyse zu berücksichtigen sind.

Im Nachhinein ist es ungewiss und heute wahrscheinlich auch nicht mehr nachzuvollziehen, ob bei der Entwicklung der Erhebungsinstrumente des Sozio-oekonomischen Panels die theoretischen Vorstellungen Essers in irgendeiner Form be- 
rücksichtigt wurden, allerdings werden einige interessante Indikatoren seit 1984 erhoben. Der beabsichtigte Test des Kausalmodells erfolgt also in Form einer Sekundäranalyse auf Basis der SOEP-Daten. Daher ist zu prüfen, inwieweit sich Indikatoren für die einzelnen Konzepte im Datensatz verwenden lassen. Zumindest das Kernmodell (Abb. 4.4.3a), bestehend aus dem Modell der Sozialintegration (Kulturation, Platzierung, Interaktion, Identifikation) und den zu erklärenden Einbürgerungs- bzw. Abwanderungsvorgängen, sollte erhoben worden sein. Ob darüber hinaus auch das Prozessmodell in seiner zeitlichen Abfolge zu testen sein wird, kann dann in einem zweiten Schritt geklärt werden.

Die Durchsicht der Variablendokumentation zeigt, dass in der Fülle an Variablen für das Kernmodell und den zu erklärenden Sachverhalt die nötigen Informationen vorhanden sind, wenn auch einige Einschränkungen hinzunehmen sind. Hinsichtlich des Zeitpunkts der Einbürgerung sind die Informationen etwas unzureichender, da das Jahr der Einbürgerung nur 2002 erfasst wurde und die ansonsten verwendete Frage nach dem Zeitpunkt des Erwerbs der deutschen Staatsangehörigkeit nur die Antwortvorgaben seit Geburt und erst später erworben vorsieht.

Als Indikator der Kulturation oder kognitiven Assimilation bietet sich die Bewertung der persönlichen Deutschkenntnisse durch den Respondenten selbst an. Darüber hinausgehende Informationen zu den kulturellen Kenntnissen und Fertigkeiten der Personen mit Migrationshintergrund finden sich im Fragebogen keine, die auch nur annähernd so kontinuierlich erhoben wurden. Die Angabe zur bevorzugten Sprache für die Zeitungslektüre, weist deutlich geringere Fallzahlen auf und ist eng mit dem Mediennutzungsverhalten verbunden, was im Sinne der Kulturation in Deutschland eine zusätzliche Dimension in den Frageninhalt einbringen würde. Gleiches gilt bei höheren Fallzahlen für die Frage nach deutschen vs. traditionellen Kochgewohnheiten und die bevorzugte Sprache beim Musikhören.

\section{Frage:}

Wie gut können Sie nach Ihrer eigenen Einschätzung deutsch sprechen?

Und schreiben?

Antwortvorgaben und Kodierung:

sehr gut $=1$, gut $=2$, es geht $=3$, eher schlecht $=4$, gar nicht $=5$ 
Für alle drei Fragen ist noch anzumerken, dass es noch weitere Sprachen oder kulturelle Traditionen gibt, die jenseits der Entscheidung zwischen deutschen und herkunftsbezogenen Ausdrucksformen liegen. Wenn es um die reine kulturelle Annäherung an die Aufnahmegesellschaft geht, dann dürfte die vorliegende subjektive Bewertung der Deutschkenntnisse eine verwendbare Annäherung an das Konstrukt Kulturation darstellen.

Im Falle der Platzierung ist die Sachlage etwas komplizierter, da die hierarchische Positionierung in einer Gesellschaft als Ausdruck der Anerkennung bestimmter Merkmale und Leistungen betrachtet werden kann und gesellschaftliche Positionen sehr unterschiedlichen Bereichen zugeordnet werden können. Esser diskutiert deshalb als spezifische Variablen für diese Form der Sozialintegration Einkommen, Berufsprestige, Bildungsabschlüsse, De-Segregation, die Verleihung von Rechten, vertikale Mobilität und auch Entscheidungen auf Märkten (z.B. Heiratsmarkt) allgemein. Im Rahmen des Prozessmodells betont er aber vor allem die berufliche Position (vgl. Esser 1980, 221,231; ders. 2001, 9-10).

Im Rahmen der Erklärung von Abwanderung und Einbürgerungsvorgängen haben sich speziell arbeitsmarktbezogene Sachverhalte als erklärungskräftig erwiesen und Bildungsvariablen mussten als eher uneindeutig eingestuft werden. Dem entsprechend ist Bildung „kein Status-Plus mehr, sondern bietet lediglich eine bessere Ausgangssituation im Verteilungskampf um das knapper werdende Gut „Arbeit“““ (Hoffmeyer-Zlotnik/Geis 2003, 126).

Die Variable De-Segregation ist m. E. nur schwer von dem Modellbestandteil Umgebung zu trennen, während Erfolg auf dem Heiratsmarkt starke Verbindungen zur Interaktion aufweist. Letzterer dürfte auch im Sinne einer Operationalisierung nicht einfach zu handhaben sein, da schwer zu entscheiden ist, was Erfolg ist und wann er eingetreten sein sollte. Obendrein müssten dauerhafte und zeitweilige Partnerschaften ohne Trauschein in Verhältnis zu Ehen und Scheidungen gesetzt werden. Die Verleihung von Rechten stellt ein weiteres Problem dar, weil sie in Form der Einbürgerung als Teil der abhängigen Variable zu betrachten ist. Die Einbürgerung stellt zweifellos eine Positionsvergabe dar, wie sie für die Platzierung charakteristisch ist. Im Rahmen des Modelltests ist sie der bis dahin erfolgten Sozi- 
alintegration allerdings nachgelagert und repräsentiert einen weiteren Schritt zu einer nachhaltigen Sozialintegration. Einbürgerung schließt die Sozialintegration nicht $a b$, sondern erweitert den Handlungsspielraum. Umgekehrt bewirkt eine Abwanderung nicht unbedingt die Aufgabe aller sozialen Bezüge zur ehemaligen Aufnahmegesellschaft.

Unmittelbar mit der Befriedigung individueller Bedürfnisse ist das Einkommen in modernen Gesellschaften verbunden. Als solches ist es als ein guter Indikator der Platzierung in den industriellen Gesellschaften anzusehen, allerdings von seiner Zusammensetzung her betrachtet deutlich komplexer als andere in Frage kommende Variablen. Als Einkommen kann die Gesamtheit der wirtschaftlichen Werte bezeichnet werden, die einer Person in einem definierten Zeitraum zufließen. Im SOEP werden die Einkünfte getrennt nach selbständiger und nicht-selbständiger Tätigkeit erfasst. Hinzu kommen noch Transfers, wie z.B. staatliche Unterstützungen, Renten, Erbschaften bis hin zu Lotteriegewinnen, die zum Teil bei NichtSelbständigen in das Nettoeinkommen eingerechnet werden. In Form des Erwerbseinkommens kann das Einkommen als Ausdruck des Erfolgs auf dem Arbeitsmarkt gewertet werden, das noch dazu über unterschiedliche gesellschaftliche Bereiche hinweg vergleichbar ist. Die Höhe der Einkünfte ist dabei als zweitrangig zu betrachten, wesentlicher ist die Relation zu den Einkommen der anderen Gesellschaftsmitglieder. Darüber hinaus kann Einkommen, das für eine mehr oder weniger regelmäßige Tätigkeit gezahlt wird, auch als wichtiger Faktor der Einbindung in eine Gesellschaft interpretiert werden. Dies gilt aber nicht für alle Einkommensarten, da beispielsweise Renten oder Erbschaften unabhängig vom jeweiligen Aufenthaltsort zufließen und dadurch nicht in gleicher Form Bindungen entstehen lassen. Alle Einkommensarten, denen die Komponente der Einbindung in einen sozialen Kontext fehlt, eignen sich aus diesem Grund nicht zur Messung der aktuellen Platzierung.

Auf Basis der SOEP-Daten wird daher nur das jeweilige Erwerbseinkommen als einer der Indikatoren der Platzierung verwendet. Damit Vergleichbarkeit zwischen den einzelnen Jahrgängen ermöglicht werden kann, ohne aufwändige Gewichtungen über die Kaufkraft durchzuführen, wird anstelle des Einkommens eine Rang- 
ordnung der Einkünfte aus Erwerbsarbeit aller SOEP-Teilnehmer für das jeweilige Jahr gebildet.

In der sozialwissenschaftlichen Analyse sozialstruktureller Unterschiede finden an Tätigkeitsmerkmalen orientierte Klassifikation und Rangordnungen von Berufen häufige Verwendung und im SOEP lassen sich einige Variablen finden, die als Indikatoren der Platzierung verwendet werden könnten. Den Beruf als Status-Variable zu betrachten ist ein gängiges Verfahren, um die hierarchische Position innerhalb der Gesellschaft zu bestimmen. Die Definitionen von Berufen und der Status, der mit ihnen verbunden wird, sind von Gesellschaft zu Gesellschaft unterschiedlich. Mit dem International Standard Classification of Occupations (ISCO) steht ein international anerkannter Maßstab zur Verfügung, Berufsgruppen über die ausgeübte Tätigkeit zu klassifizieren. Auf der ISCO-Klassifikation basieren wiederum z.B. die Standard International Occupational Prestige Scale (SIOPS), der International Socio-Economic Index of Occupational Status (ISEI) und die für die Bundesrepublik entwickelte Magnitude-Prestige-Skala (MPS), die alle in den SOEP-Daten zu finden sind (Treiman 1977; Ganzeboom/De Graaf/Treiman/de Leeuw 1992; Wegener 1988). Diese Prestige-Skalen sind als Rangordnungen der ISCO-Klassifikation konstruiert worden. Von Klassifizierungen und Rangordnungen für internationale Vergleiche kann nicht erwartet werden, dass sie die jeweiligen länderspezifischen Besonderheiten in all ihren Facetten widerspiegeln, daher sind deren Übertragbarkeit auf Gesellschaften und auch der langfristigen Verwendbarkeit innerhalb einer Gesellschaft Grenzen gesetzt (vgl. Hoffmeyer-Zlotnik/Geis 2003). Die SIOPS und die ISEI sind für internationale Vergleiche entwickelte Klassifikationen, die von Wegener für Deutschland auf Grundlage des ISCO-68 konzipierte MPS, wurde bisher noch nicht auf die neuere Variante, den ISCO-88, umgestellt und kann deshalb nicht unbedingt als den Erfordernissen angemessen angesehen werden.

Als nationale Alternative, die ebenfalls im SOEP zur Verfügung steht, hat Jürgen H.P. Hoffmeyer-Zlotnik 1993 eine auf der Stellung im Beruf basierende fünfstufige Prestige-Skala der Autonomie des beruflichen Handelns vorgestellt, mit der anhand von Tätigkeitsmerkmalen eine Zuordnung über die jeweilige Handlungsautonomie des Berufstätigen getroffen wird. Der einfache und plausible Grundgedanke ist, dass von einer höheren Handlungsautonomie im Beruf ein höheres soziales Pre- 
stige abgeleitet werden kann (Hoffmeyer-Zlotnik/Warner 1998). Die Gruppierung erfolgt in fünf ordinale Kategorien, von $1=$ niedriger bis $5=$ hohe Arbeitsautonomie (Hoffmeyer-Zlotnik 1993). ${ }^{43}$ Diese über die Tätigkeitsmerkmale begründete Prestige-Skala soll leicht modifiziert als Indikator der Platzierung dienen. Die Modifikation betrifft ein Problem, das mit allen Skalen und Klassifizierungen, die auf dem ausgeübten Beruf aufbauen, verbunden ist. Personen, die keinen Beruf ausüben, können definitionsgemäß nicht zugeordnet werden, was die Zahl der in die Analyse eingehenden Fälle reduziert. Eine Ersetzung der Werte durch Mittelwerte oder statistische Schätzverfahren erscheint in diesem Zusammenhang nicht sinnvoll, weil damit eine Prestigezuweisung erfolgen würde, die der Konstruktion der Skalen widerspräche (vgl. Engel/Reinecke 1994, 260f). Der Logik der Prestigeskalen folgend, wird deshalb allen Personen, die arbeitssuchend gemeldet sind, der Wert 0 gleich kein gegenwärtiges berufliches Prestige zugewiesen. Hintergrund dieser Setzung ist die Feststellung, der zufolge niemand ohne aktuelle berufliche Beschäftigung, über die mit der Tätigkeit verbundene Autonomie, soziales Prestige ableiten kann. ${ }^{44}$

\section{Autonomie beruflichen Handelns nach Klassifikation der beruflichen Stellung:

$\begin{array}{ll}1=\text { niedrig } & \text { ungelernte und angelernte Arbeiter } \\ 2 & \text { z.B. Landwirte unter } 10 \text { ha, Beamte einf. Dienst, Verkäufer } \\ 3 & \text { z.B. Selbständige bis ein Mitarb., Beamte mittl. Dienst } \\ 4 & \text { z.B. Beamte gehob. Dienst, wiss. Mitarbeiter, Arzt mit 2-9 Mitarb. } \\ 5=\text { hoch } & \text { z.B. Beamte höherer Dienst, Direktoren, Vorstände gr. Verbände }\end{array}$

Für die Interaktion steht im Fragenprogramm des Sozio-oekonomischen Panels eine nicht ganz so große Auswahl an potentiellen Operationalisierungen zur Verfügung. In den Jahren zwischen 1988 und 2001 wurde die Herkunft der besten drei Freunde fast alle zwei Jahre erfragt, der Kontakt zu Deutschen allgemein ab 1985 bis 1999 und der Besuch durch Deutsche in der eigenen Wohnung bzw. der Besuch in deren Wohnung ab 1985 bis 2003 ebenfalls im Zwei-Jahres-Rhythmus. Die Frage nach dem Kontakt zu Deutschen ist für die Messung der Interaktion zu wenig

43 In einem Vergleich der Autonomie beruflichen Handelns mit dem SIOPS, ISEI und MPS wurde eine hohe Übereinstimmung auf Basis deutscher Daten festgestellt (vgl. Wolf 1995).

44 Personen, die nicht unmittelbar am Erwerbsleben teilnehmen, wurden damit bei der Analyse nicht berücksichtigt. Alternativ wurde zur Kontrolle auch Pensionären und Rentnern der Wert 0 für kein gegenwärtiges Berufsprestige zugewiesen, was in den Strukturgleichungsmodellen lediglich zu einer geringfügigen Stabilisierung der Modelle führte. 
differenzierend, da in einer Mehrheits-Minderheitsrelation wie in der Bundesrepublik Deutschland, der Kontakt zur Mehrheitsgesellschaft nur in Ausnahmefällen vollständig vermieden werden kann. Die Frage nach den wichtigsten Freunden ist hingegen sehr spezifisch, da private Interaktionen deutlich über engsten Freundekreis hinausgehen. Von Deutschen besucht zu werden und diese in ihrer Wohnung zu besuchen, ist eine Variante, die darauf abzielt, soziale Interaktion zwischen Nähe und anonymen Alltagskontakten zu erfassen. Für diesen Indikator spricht darüber hinaus, dass Esser in seiner Pfadanalyse in „Aufenthaltsdauer und die Eingliederung von Wanderern“ nahezu eine identische Operationalisierung des Kontakts verwendete (vgl. Esser 1981, 95).

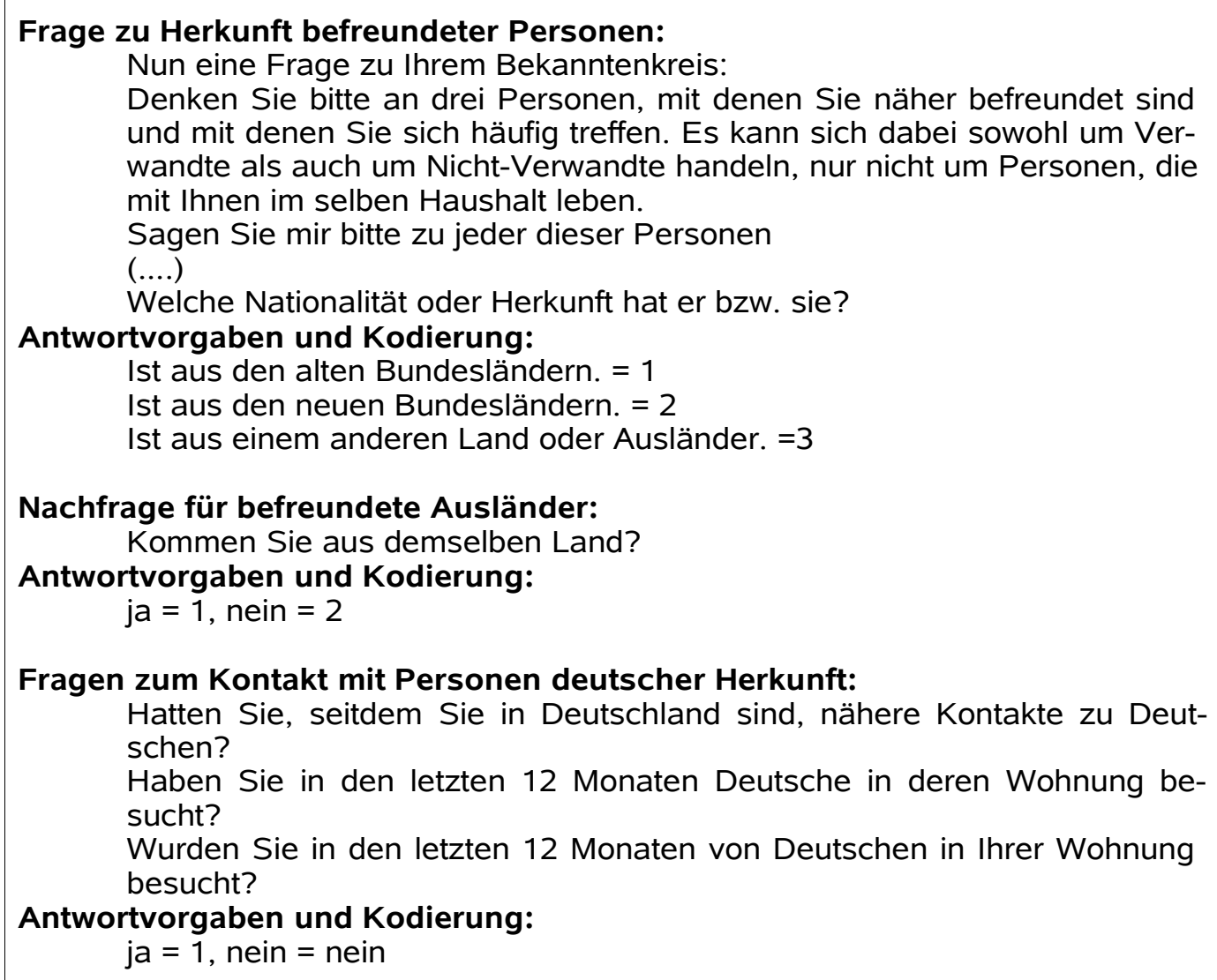

Im Hinblick auf die Erfassung der emotionalen Identifikation mit der Aufnahmeund der Herkunftsgesellschaft sind zwei Fragen in den Personenfragebögen des Sozio-oekonomischen Panels enthalten, die als Indikatoren verwendet werden kön- 
nen. Die Frage zur deutschen Identität zielt dabei eindeutig auf die emotionale Verbindung und wird in dieser Form seit 1985 erhoben. Im Falle der emotionalen Verbundenheit mit der Herkunftsgesellschaft ist die Frage bis 1995 als Pendant zur deutschen Identifikation gestellt worden und ab 1997, offensichtlich mit Rücksicht auf die Angehörigen der zweiten und dritten Generation, als Frage nach der Verbundenheit mit dem Land, aus dem der Befragte selbst oder seine Familie nach Deutschland gekommen ist. Als Nachteil muss hier angesehen werden, dass mit der Umstellung 1997 durch die Begriffe Land und Familie das Item eine etwas andere Konnotation aufweist.

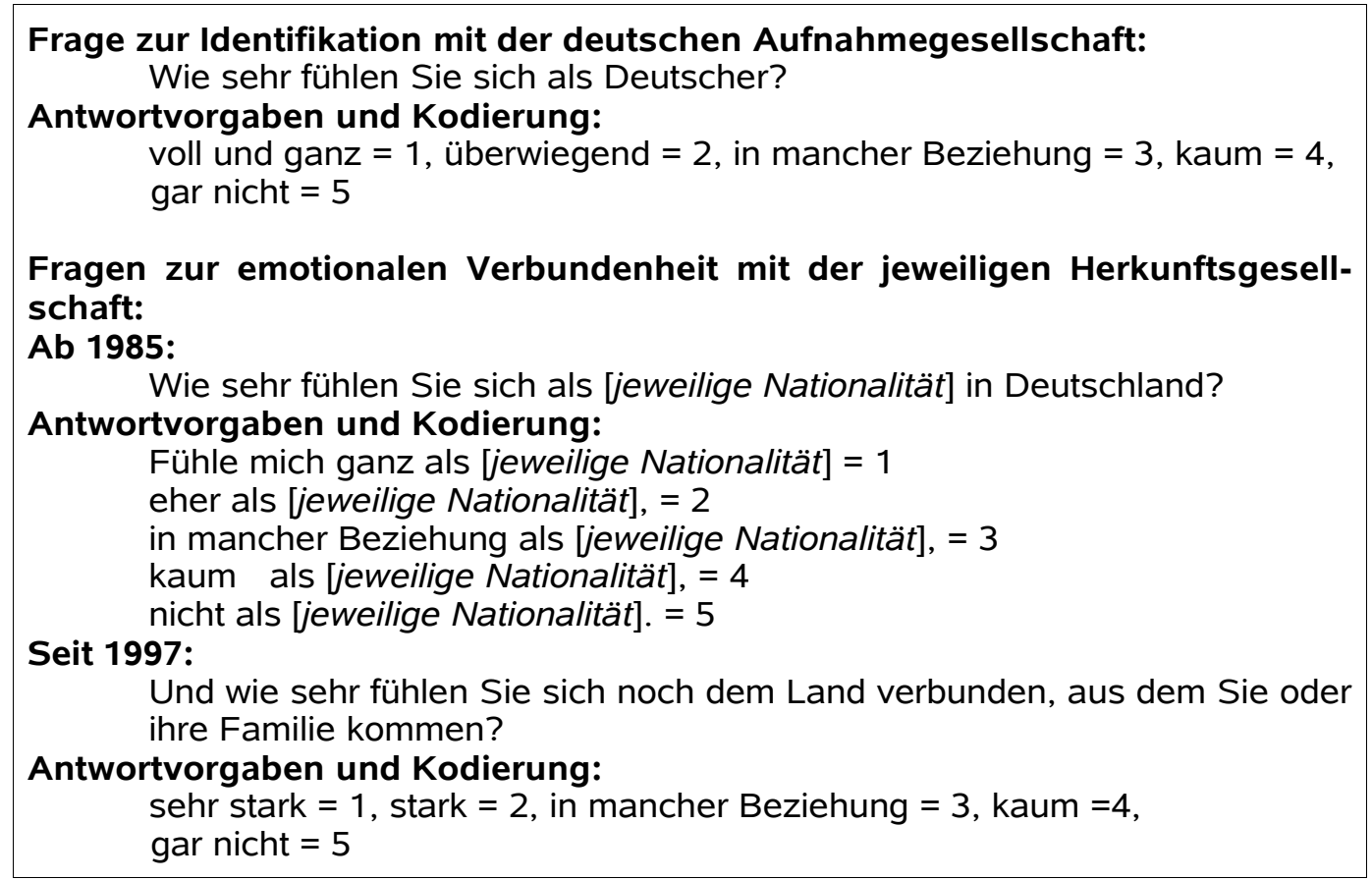

Für die Operationalisierung der zu erklärenden Einbürgerungs- und Abwanderungsvorgänge wurde eine Variable erzeugt, die drei Ausprägungen (Einbürgerung, keine Änderung, Abwanderung) aufweist. Die Abwanderungsinformation bezieht sich auf die Dokumentation der Ausfallgründe, in der auch der Ausfallgrund ins Ausland verzogen enthalten ist. Problematisch hieran ist, dass es auch eine Reihe von ungeklärten Ausfallgründen gibt, die möglicherweise noch zum Teil Abwanderungen enthalten. Die Information über eine Einbürgerung wurden zum einen aus dem Wechsel der Staatsangehörigkeit bzw. aus dem zusätzlichen Erwerb der deutschen Staatsangehörigkeit entnommen und zum anderen ergänzt über die Variable Zeitpunkt des Erwerbs der deutschen Staatsangehörigkeit. Ursprünglich sollte darü- 
ber hinaus die Dauer bis zum Eintritt des jeweiligen Ereignisses in die Variable mit einfließen, doch leider waren für den betrachteten Zeitraum nicht ausreichend Fälle für eine aufgefächerte Kodierung vorhanden. In Tabelle 5.1.2.1a sind abschließend die ausgewählten Indikatoren der einzelnen Konstrukte aufgeführt.

Tabelle 5.1.2.1a: Übersicht zu den ausgewählten Items und Variablen

\begin{tabular}{|c|c|c|}
\hline Konstrukt & $\begin{array}{c}\text { Itemkurz- } \\
\text { bezeich- } \\
\text { nung }\end{array}$ & Item bzw. Variable \\
\hline \multirow{2}{*}{ Kulturation } & kultura 1 & $\begin{array}{l}\text { Wie gut können Sie nach Ihrer eigenen Einschätzung deutsch } \\
\text { sprechen? }\end{array}$ \\
\hline & kultura 2 & Und schreiben? \\
\hline \multirow{2}{*}{ Platzierung } & platzier 1 & Autonomie des beruflichen Handelns (incl. Arbeitsuchende) \\
\hline & platzier 2 & Arbeitseinkommen \\
\hline \multirow{2}{*}{ Interaktion } & inter 1 & $\begin{array}{l}\text { Haben Sie in den letzten } 12 \text { Monaten Deutsche in deren Woh- } \\
\text { nung besucht? }\end{array}$ \\
\hline & inter 2 & $\begin{array}{l}\text { Wurden Sie in den letzten } 12 \text { Monaten von Deutschen in Ihrer } \\
\text { Wohnung besucht? }\end{array}$ \\
\hline \multirow[b]{2}{*}{ Identifikation } & ident 1 & Wie sehr fühlen Sie sich als Deutscher? \\
\hline & ident 2 & $\begin{array}{l}1985 \text { bis 1995: } \\
\text { Wie sehr fühlen Sie sich als [jeweilige Nationalität] in Deutsch- } \\
\text { land? } \\
\text { ab 1997: } \\
\text { Und wie sehr fühlen Sie sich noch dem Land verbunden, aus } \\
\text { dem Sie oder ihre Familie kommen? }\end{array}$ \\
\hline $\begin{array}{l}\text { Einbürge- } \\
\text { rungs-Ab- } \\
\text { wanderungs- } \\
\text { Dimension }\end{array}$ & ead & $\begin{array}{l}\text { Einbürgerung: } \\
\text { Wechsel zur deutschen Staatsangehörigkeit oder zusätzlicher } \\
\text { Erwerb der deutschen Staatsangehörigkeit } \\
\text { keine Änderung: } \\
\text { Zielperson ohne Einbürgerung oder Abwanderung } \\
\text { Abwanderung: } \\
\text { Ausfallgrund: Ins Ausland verzogen }\end{array}$ \\
\hline
\end{tabular}

Für die hier betrachteten ausschließlich an Immigranten gerichteten Fragen bleibt insgesamt nachzutragen, dass die Reihenfolge im Fragebogen - als erstes die Identifikationsitems, danach die Spracheinschätzung und zuletzt die Kontaktfra- 
gen - von 1984 bis 1995 beibehalten wurde. Im Jahr 1997 wurde diese Abfolge in zuerst Sprache, dann Kontakt und zuletzt Identifikation abgeändert. In der darauffolgenden Erhebung 1999 wurden zunächst die Kontakt-, danach die Sprach- und zuletzt die Identifikationsfragen gestellt. Die neue Reihenfolge wurde seitdem nicht weiter verändert. Es kann leider nicht abgeschätzt werden, ob es zu Reihenfolgeeffekten gekommen ist, da keine Kontrollgruppen erhoben wurden. Die Durchsicht der Erhebungsinstrumente ergab aber nicht nur für die Frageformulierungen und Reihenfolge der Items Änderungen, sondern auch die Filterführung wurde geändert. In Abhängigkeit von den zuvor gestellten Fragen nach Staatsangehörigkeit und dem Aufenthalt in Deutschland wurden unterschiedlichen Gruppen die Fragen zur Sprache, zum Kontakt und zur Identifikation gestellt.

Tabelle 5.1.2.1b: Auswirkungen der Filterführung auf die Zusammensetzung der Antwortenden (1997-2004)

\begin{tabular}{|c|c|c|c|c|c|}
\hline Befragtengruppe & 1985-1995* & 1997 & 1999 & 2001 & 2003 \\
\hline ohne deutsche Staatsangehörigkeit & $\begin{array}{l}\text { Identifikation, } \\
\text { Sprache, } \\
\text { Kontakt }\end{array}$ & $\begin{array}{c}\text { Sprache, } \\
\text { Kontakt, } \\
\text { Identifikation }\end{array}$ & $\begin{array}{l}\text { Kontakt, } \\
\text { Sprache, } \\
\text { Identifikation }\end{array}$ & $\begin{array}{c}\text { Kontakt, } \\
\text { Sprache, } \\
\text { Identifikation }\end{array}$ & $\begin{array}{c}\text { Kontakt, } \\
\text { Sprache, } \\
\text { Identifikation }\end{array}$ \\
\hline $\begin{array}{l}\text { mit deutscher Staatsangehörigkeit } \\
\text { und in Deutschland geboren }\end{array}$ & nicht erfasst & $\begin{array}{c}\text { Fragen } \\
\text { übersprungen }\end{array}$ & $\begin{array}{c}\text { Fragen } \\
\text { übersprungen }\end{array}$ & $\begin{array}{c}\text { Fragen } \\
\text { übersprungen }\end{array}$ & nicht erfasst \\
\hline $\begin{array}{l}\text { mit deutscher Staatsangehörigkeit, } \\
\text { nicht in Deutschland geboren } \\
\text { und bereits vor } 1984 \text { in Deutschland } \\
\text { wohnend }\end{array}$ & nicht erfasst & $\begin{array}{c}\text { Fragen } \\
\text { übersprungen }\end{array}$ & $\begin{array}{c}\text { Fragen } \\
\text { übersprungen }\end{array}$ & $\begin{array}{c}\text { Fragen } \\
\text { übersprungen }\end{array}$ & nicht erfasst \\
\hline $\begin{array}{l}\text { mit deutscher Staatsangehörigkeit, } \\
\text { aber nicht in Deutschland geboren } \\
\text { und erst nach } 1984 \text { in Deutschland } \\
\text { wohnend }\end{array}$ & nicht erfasst & $\begin{array}{c}\text { Sprache, } \\
\text { Kontakt, } \\
\text { Identifikation }\end{array}$ & $\begin{array}{l}\text { Sprache, } \\
\text { Identifikation }\end{array}$ & $\begin{array}{l}\text { Sprache, } \\
\text { Identifikation }\end{array}$ & nicht erfasst \\
\hline $\begin{array}{l}\text { mit Geburt deutscher Staatsbürger } \\
\text { (nur 2003) }\end{array}$ & nicht erfasst & nicht erfasst & nicht erfasst & nicht erfasst & $\begin{array}{c}\text { Fragen } \\
\text { übersprungen }\end{array}$ \\
\hline $\begin{array}{l}\text { zu einem späteren Zeitpunkt die } \\
\text { deutsche Staatsangehörigkeit } \\
\text { erworben (nur 2003) }\end{array}$ & nicht erfasst & nicht erfasst & nicht erfasst & nicht erfasst & $\begin{array}{l}\text { Sprache, } \\
\text { Identifikation }\end{array}$ \\
\hline
\end{tabular}

Quelle: SOEP Wellen 2, 4, 6, 8, 10, 12, 14, 16, 18 und 20

Wie aus Tabelle 5.1.2.1b hervorgeht betreffen die Veränderungen nur Personen, die über die deutsche Staatsangehörigkeit verfügen und nicht die Gruppe der Per- 
sonen ohne deutsche Staatsangehörigkeit. Bedingt durch diese Filterführung ist unter Berücksichtigung der geringen Fallzahl an eingebürgerten Personen vor 1995 mit leichten Verzerrungen bzw. Differenzen zwischen den entsprechenden Wellen zu rechnen. ${ }^{45}$

\subsubsection{Konsequenzen für die empirische Analyse}

Für die geplante Sekundäranalyse auf Basis des Sozio-oekonomischen Panels war zunächst eine eingehende Prüfung notwendig, ob im Fragenprogramm der Erhebungen angemessene Indikatoren der Konstrukte zu finden sind. Die Durchsicht der Variablen hat sowohl für die einzelnen Elemente des Kernmodells der Sozialintegration als auch für die Einbürgerungs- und Abwanderungsvorgänge akzeptable Indikatoren ergeben. Der Aspekt der Assimilations-Dissimilations-Perspektive wird hierbei nur zum Teil erfasst, nämlich unmittelbar in den Indikatoren der Identifikation und implizit auch in Hinsicht auf die Interaktion.

Aber es war nicht nur zu prüfen, ob entsprechende Indikatoren in den Datensätzen enthalten sind, zudem war im Hinblick auf die Prüfung des Prozesses der Sozialintegration und seiner Auswirkungen auf die zu erklärenden Vorgänge die Reihenfolge und Stichprobenzusammensetzung relevant. Auch diese Prüfung hat zu einem zumindest zufriedenstellenden Ergebnis geführt. Die Indikatoren der Kulturation, Interaktion und Identifikation sind von 1985 regelmäßig alle zwei Jahre erhoben worden, die notwendigen Variablen für Platzierung und Abwanderung finden sich in allen Wellen ab 1985, lediglich die Frage zur deutschen Staatsangehörigkeit ist explizit erst seit 1996 Teil des jährlichen Fragenprogramms. Inwieweit es für die Analysen möglich wäre, die Angaben zum Erwerb der deutschen Staatsangehörigkeit valide durch die Variablen Jahr der Einbürgerung (nur 2002) und Staatsangehörigkeit (1984-2004) zu ergänzen, muss nicht weiter erörtert werden, da der Zeitabschnitt von 1996 bis 2004 für den Test des Erklärungsmodells als ausreichend

45 Für die Betrachtung des Integrationsverlaufs von Personen mit deutscher Staatsangehörigkeit und ethnisch-nicht-deutscher Herkunft würden die Sprunganweisungen in den Personenfragebögen jedoch erhebliche Einschränkungen mit sich bringen, da das Kriterium der Nationalität im Vordergrund steht. 
bewertet werden kann. Allerdings sind dadurch die Möglichkeiten einer Prüfung der Dauer des Prozesses und der Relevanz von Zeitabständen sehr eingeschränkt.

Aus dem Überblick zur Anzahl der Personen mit ausländischer Staatsangehörigkeit in den einzelnen Panelwellen (Tab. 5.1.1a) ist zu erkennen, dass trotz Ergänzungsstichproben die für Längsschnittstudien dieser Laufzeit nicht zu vermeidende Panelmortalität auch den Umfang der betrachteten ausländischen Subgruppe im SOEP erheblich reduziert hat. In Anbetracht der Dauer von 21 Jahren und der ansonsten für die Bundesrepublik festgestellten hohen Abwanderungsrate (vgl. Kap. 3.1.1) sind Panelausfälle in einer Größenordnung von 50\%-60\% noch als eher gering einzustufen. Dies gibt Anlass dazu, über die Zusammensetzung der Stichproben im Vergleich zur Grundgesamtheit einige weitergehende Überlegungen anzustellen, denn in einigen sozialwissenschaftlichen Untersuchungen, die sich auf die SOEP-Daten beziehen, wird davon ausgegangen, dass die Migrantenstichprobe der jeweiligen Grundgesamtheit entspricht (z.B. Jankowitsch/Klein/Weick 2000, Constant/Zimmermann 2003, Dustmann 2001, Fertig 2004). Hingegen sieht Frick die Repräsentativität der Immigrantenstichprobe in den Jahren 1984-1993 als nicht gegeben an (vgl. Frick 2004, 9).

Aber die Frage danach, ob eine Stichprobe ein adäquates Abbild der Grundgesamtheit liefern kann, ist nicht unabhängig von der inhaltlichen Fragestellung zu betrachten. Im Kontext von Abwanderung und Einbürgerung kann die langfristige oder auch nur vorübergehende Teilnahme an einer Panelstudie im Sinne des hier vorgestellten Erklärungsmodells als zusätzliche Einbindung in die Mehrheitsgesellschaft gesehen werden und damit eine geringere Abwanderungsrate als unter Nicht-Teilnehmern bewirken. Zumal zusätzlich die Maßnahmen zur Panelpflege (Geschenke und Informationen zu den SOEP-Ergebnissen) und das angestrebte persönliche Verhältnis zwischen Interviewern und Respondenten einen bindenden Effekt erzielen sollen. Um abschätzen zu können, ob ein solcher Paneleffekt existiert und welches Ausmaß er gegebenenfalls hätte, wäre allerdings eine Kontrollgruppe ausländischer Personen ohne jeglichen Kontakt zu den erhebenden Institutionen erforderlich. Denn jeder Versuch der Kontaktaufnahme kann entsprechend der sozialen Produktionsfunktion als potentielle soziale Anerkennung durch die Aufnahmegesellschaft interpretiert werden. Noch dazu werden über das Fragenprogramm des 
SOEP implizit relevanten Informationen vermittelt, da im Rahmen der Kommunikation zwischen Interviewer und Respondent ganz praktische und im alltäglichen Leben hilfreiche Sachverhalte wie z.B. Transferleistungen, Organisationsmöglichkeiten und Varianten des Aufenthaltsstatus zumindest thematisiert werden müssen. Sofern die damit verbundenen Paneleffekte existieren, beeinträchtigen sie auch die Verallgemeinerbarkeit der Ergebnisse auf die Gruppe der Immigranten und ihrer Nachkommen insgesamt. Aber selbst in der Bereitschaft an einer Panelbefragung teilzunehmen, kann schon eine Verzerrung der Stichprobe in Richtung der Aufenthaltsabsichten und des Aufenthalts begründet sein. Für alle Personen mit geringen Deutschkenntnissen kommt, sofern sie nicht griechisch, italienisch, serbokroatisch oder türkisch sprechen, als Selektionskriterium hinzu, dass keine Erhebungsinstrumente in der jeweiligen Muttersprache vorliegt. Somit dürften Analysen der Sozialintegration für diese Gruppen im Vergleich zu den klassischen Arbeitsmigranten bedingt durch die impliziten Auswahlkriterien günstiger ausfallen. Trotz der genannten Gründe für mögliche Verzerrungen ist das SOEP für Analysen zur Sozialintegration im Zusammenhang mit den Abwanderungs- und Einbürgerungsvorgängen in der Bundesrepublik Deutschland als die einzige Datenbasis anzusehen.

Die potentiellen aber auch wahrscheinlichen Einschränkungen der Verallgemeinerbarkeit beeinträchtigen insgesamt auch den geplanten Modelltest. Gleichzeitig bieten die Daten des Sozio-oekonomischen Panels aber die Möglichkeit, das Erklärungsmodell auf Basis der Sozialintegration hinsichtlich der Kausalstruktur und auch bezogen auf die zeitliche Entwicklung in Form des Prozessmodels einer Überprüfung zu unterziehen. Da kein Datensatz vorliegt, in dem die für den geplanten Modelltest notwendigen Informationen in angemessenerer Form erhoben wurden, wird die Prüfung des Modells trotz der möglichen Verzerrungen an den SOEP-Daten vorgenommen. Die genannten Einschränkungen sind allerdings bei der Bewertung der Ergebnisse in Rechnung zu stellen. 


\subsection{Auswahl des statistischen Analysemodells zur Prüfung der kausalen Beziehungen an den Daten}

Ziel statistischer Analysen ist es, Informationen zusammenzufassen und orientiert an den Ergebnissen Schlussfolgerungen zu den durch empirische Daten repräsentierten Sachverhalten zu ermöglichen. Vor dem Hintergrund der jeweiligen theoretisch-inhaltlichen Fragestellung und mit Rücksicht auf die Datenstruktur kann aber nicht jedes statistische Analysemodell gleichermaßen als geeignet angesehen werden, da die Auswahl unangemessener statistischer Modelle unzutreffende Schlussfolgerungen zur Folge haben kann.

Ausgehend von der zu prüfenden Kausalstruktur des spezifizierten Erklärungsmodells kommen nur solche Analyseverfahren in Betracht, mit denen sich die Beziehungen der einzelnen theoretischen Konstrukte untereinander auch statistisch modellieren lassen. In den Anwendungsbeispielen des Assimilationsmodells verwendeten Esser (1981; 1982) und Hill (1984) Pfadanalysen auf Basis empirischer Korrelationen einzelner Indikatoren bzw. der Faktorkorrelationen latenter Konstrukte aus explorativen Faktorenanalysen mit restringierter Faktorenzahl. Das Verfahren der Pfadanalyse kann als Erweiterung des linearen Regressionsmodells aufgefasst werden, bei dem gerichtete Beziehungen sowohl zwischen exogenen und endogenen als auch unter den endogenen Variablen in einem Modell simultan geprüft werden können. Die Beziehungen der einzelnen Elemente des theoretischen Modells lassen sich dementsprechend in Form linearer Regressionsgleichungen ausdrücken, die wiederum gemäß dem Fundamentaltheorem der Pfadanalyse als

$$
r_{i j}=\sum_{q} p_{i q} * r_{q j}
$$

Kombination von Korrelationskoeffizienten beschrieben werden können (vgl. Seibel/Nygreen 1972; Opp/Schmidt 1976). In den verwendeten Verfahren wurden diesem Ansatz folgend die Regressions- bzw. Pfadkoeffizienten aus den zuvor ermittelten Korrelationen errechnet. Faktorkorrelationen zur Schätzung der Koeffizienten zu verwenden, ist eine Vorgehensweise, die dem Problem empirischer Sozi- 
alforschung Rechnung trägt, dass ein Teil theoretischer Konstrukte, wie z.B. Identität und Kulturation nicht direkt in der Realität beobachtet werden können. Die Messung sogenannter Faktoren oder latenter Konstrukte erfolgt über Variablen, die sich als Indikatoren der latenten Variablen interpretieren lassen. Zur statistisch angemesseneren Modellierung kausaler Beziehungen zwischen latenten Konstrukten eignen sich Verfahren, die Pfadanalyse und Faktorenanalyse zu einem statistischen Modell verbinden. Zusätzlich ergibt sich durch die Verwendung mehrerer Indikatoren für die Messung eines Konstrukts die Möglichkeit, Meßfehleranteile der gemessenen Variablen zu bestimmen. Zwei Ansätze, die die genannten Eigenschaften aufweisen, stehen zur Auswahl: Erstens die auf Tabellenanalyse mittels log-linearer Modelle beruhende latente Klassenanalyse (vgl. Andreß/Hagenaars/Kühnel 1997, 209-259) und nicht zuletzt die auf die Analyse empirischer Varianzen und Kovarianzen ausgerichteten linearen Strukturgleichungsmodelle (vgl. Engel/Reinecke 1994; Reinecke 2005). Im Hinblick auf die zeitliche Komponente des Prozesses wären Ereignisanalysen angemessener, denn im Prinzip handelt es sich bei der Einbürgerungs-Abwanderungs-Dimension um eine rechtszensierte Variable. ${ }^{46}$ Um die ereignisorientierte Datenstruktur des Sozio-oekonomischen Panels besser zu nutzen, wären Ansätze wie die Hazard-Modelle geeigneter, allerdings ist die simultane Prüfung des Strukturmodells inklusive der indirekten Effekte mit einem solchen Verfahren leider nicht möglich (vgl. Blossfeld/Hamerle/Meyer 1986).

Latente Klassenanalyse und lineare Strukturgleichungsmodelle unterscheiden sich u.a. dadurch, dass für latente Klassenanalyse kategoriale Daten unabhängig vom Skalenniveau verwendbar sind, während lineare Strukturgleichungsmodelle metrisches oder zumindest ordinales Messniveau erfordern. ${ }^{47}$ Die Beziehungen der

46 Es ist möglich, dass im Beobachtungszeitraum die analysierten Prozesse noch nicht abgeschlossen sind, dies wird als rechtszensiert bezeichnet. Wird eine Zensierung ignoriert, kann dies zu verzerrten Resultaten führen (vgl. Blossfeld/Hamerle/Meyer 1986, 72-74). Letztlich ist auch die Entscheidung über Einbürgerung bzw. Abwanderung erst mit dem Eintritt des jeweiligen Ereignisses oder dem Tod der Person abgeschlossen. Aus diesem Grund wurden im vorliegenden Fall Kontrollrechnungen mit unterschiedlichen Zeitabständen durchgeführt, die im Hinblick auf die kausale Struktur keine nennenswerten Veränderungen ergaben.

47 Bei ordinalem Messniveau sind Erwartungswerte und Varianzen nur unter spezifischen Annahmen schätzbar. Bei Variablen mit nur zwei Ausprägungen ist es sogar grundsätzlich unmöglich, Varianzen und Erwartungswerte gleichzeitig zu schätzen. Diese Einschränkung trifft in den folgenden Analysen auf die Kontaktvariablen zu. Aus diesem Grunde basieren die geschätzten Struktugleichungsmodelle auf polychorischen Korrelationen, die auch im Falle von dichotomen Variablen geschätzt werden können (vgl. Reineke 2005, 29-35). 
einzelnen Konstrukte des Modells der Sozialintegration untereinander und mit den Einbürgerungs- und Abwanderungsvorgängen lässt sich mit beiden Ansätzen analysieren. Allerdings ergibt sich im Falle der log-linearen Modelle ein anwendungstechnisches Problem. Die Zahl der Zellen in einer log-linearen Tabellenanalyse ist gleich dem Produkt der Zahl an Kategorien der verwendeten Variablen, was mit der Anzahl zu schätzender Modellparameter korrespondiert. Die Analyse der für das theoretische Erklärungsmodell erforderlichen Tabellen mittels log-linearer Modelle muss bedingt durch die Größe der Tabellen als nicht mehr adäquat angesehen werden. Alternativ wäre es möglich, die vorhandenen ordinalen Informationen zu wenigen Kategorien zusammenzufassen, dies wäre aber mit Informationsverlust verbunden (vgl. Kühnel 1993; 134-135).

Letztlich ist damit der Ansatz linearer Strukturgleichungsmodelle als die angemessenere statistische Modellierung für die postulierten Beziehungen der Variablen im Erklärungsmodell anzusehen. Obwohl mit strukturprüfenden Verfahren wie lineare Strukturgleichungsmodelle oft der Anspruch, Kausalität nachweisen zu können, verbunden wird, muss dies etwas differenzierter betrachtet werden. Kausale Beziehungen lassen sich durch statistische Modellprüfung nicht nachweisen, es kann statt dessen lediglich geprüft werden, ob die den inhaltlichen Hypothesen zugrundeliegenden Annahmen über die kausalen Zusammenhänge mit den empirischen Daten zu vereinbaren sind oder mit diesen nicht in Einklang stehen (vgl. Reinecke 2005, 12). Die Datenanalyse mit linearen Strukturgleichungsmodellen ist überdies geeignet, Längsschnittinformationen über die Veränderung und Stabilität von latenten und manifesten Variablen zu untersuchen, was für die Prüfung des Prozessmodells der Sozialintegration eine notwendige Voraussetzung darstellt. Insgesamt gesehen sollte also eine Analyse der Daten des Sozio-oekonomischen Panels mit dem linearen Strukturgleichungsansatz zur Prüfung der formulierten Hypothesen möglich sein und gleichzeitig eine statistisch adäquate Modellierung des Erklärungsmodells erlauben. 


\subsection{Zwischenfazit}

Ausgehend von den theoretischen Anforderungen, denen die Daten genügen müssen, wurde in diesem Kapitel geprüft, ob das Sozio-oekonomische Panel des Deutschen Instituts für Wirtschaftsforschung als Datenbasis für die Prüfung der kausalen Beziehungen von Sozialintegration zu den Einbürgerungs- und Abwanderungsvorgängen dienen kann.

Diese Überprüfung ergab, dass das SOEP eine umfangreiche Teilstichprobe in Deutschland lebender ausländischer Staatsangehöriger beinhaltet und das Forschungsdesign bestehend aus retrospektiv erhobenen Ereignisdaten und klassischer Längsschnittstudie den Erfordernissen des prozessbezogenen Erklärungsmodells sehr entgegen kommt. Im Rahmen des seit nunmehr über zwanzig Jahren laufenden Panels wurden und werden tendenziell in regelmäßigen Abständen immer wieder dieselben thematischen Fragenkomplexe den Respondenten vorgelegt, darunter sind auch Fragen, die als Indikatoren der einzelnen theoretischen Konstrukte dienen können.

Insgesamt erfüllen demzufolge die SOEP-Daten von ihrer Konzeption, der Datenstruktur und dem Fragenprogramm her die für den Modelltest notwendigen Voraussetzungen. Einschränkungen des positiven Gesamtergebnisses sind nur im Hinblick auf die Frage nach der deutschen Staatsangehörigkeit bzw. den Einbürgerungszeitpunkt und die Repräsentativität der Ausländerstichprobe hinzunehmen. Die Daten des Sozio-oekonomischen Panels können für die Prüfung des Modells der Sozialintegration und den daran anschließenden Erklärungsversuch zu den Einbürgerungs- und Abwanderungsvorgängen als angemessen angesehen werden.

Auf den theoretischen Vorgaben und der Datenbasis aufbauend wurde der Frage nachgegangen, welches statistische Analysemodell eine angemessene Umsetzung erlaubt. Esser verwendete bei seinen Anwendungen des Assimilationsmodells die Pfadanalysen zur Prüfung an den Daten. Ausgehend von den theoretischen Vorgaben und dem Aufbau der Daten, wurde sich für den linearen Strukturgleichungsansatz entschieden, der eine Verbindung von Pfad- und Faktorenanalyse in einer simultanen Schätzung ermöglicht. Dazu wird neben dem Pfad- bzw. Strukturmodell ein sogenanntes Messmodell spezifiziert, das die Verbindung von beobachteten 
und latenten Variablen beinhaltet. Insbesondere diese Eigenschaft linearer Strukturgleichungsmodelle ermöglicht es, nicht direkt beobachtbare Konstrukte, wie sie in Form von Identität und Kulturation auch Bestandteile des Erklärungsmodells sind, mittels Indikatoren statistisch angemessener in die Analyse einzubinden. 


\section{Analysen zur Erklärung der Abwanderungs- und Einbürgerungsvorgänge}

Der von Hartmut Esser entwickelte Ansatz der Sozialintegration zielt darauf ab, die Integrationstypen und darauf aufbauende makrosoziologische Entwicklungen im Verhältnis von Immigranten und Aufnahmegesellschaft zu erklären. Bezogen auf das Thema dieser Arbeit kann der Ansatz genutzt werden, um ausgehend von den Integrationsverläufen der Frage nachzugehen, warum sich Personen mit Migrationshintergrund entscheiden für immer zu bleiben, während andere das Aufnahmeland (wieder-)verlassen. In diesem Kapitel sollen auf Grundlage der Daten des Sozio-oekonomischen Panels die hierzu notwendigen Analysen vorgestellt und diskutiert werden.

Nach dem vorgestellten Modell ist die Entscheidung zur Abwanderung bzw. Einbürgerung Ausdruck der auf Integration beruhenden Möglichkeiten eingebettet in einen sozialen Kontext, die eigenen Bedürfnisse zu befriedigen. Voraussetzung für eine erfolgreiche Erklärung der Abwanderungs- und Einbürgerungsvorgänge ist, dass die Sozialintegration von Immigranten tatsächlich die postulierten Beziehungen zwischen den Subdimensionen aufweist. Insofern ist es naheliegend im ersten Analyseschritt die Beziehungen der einzelnen Elemente genauer zu betrachten. Mittels der SOEP-Daten kann dies bezogen auf unterschiedliche Zeitpunkte geschehen, da die notwendigen Indikatoren zwischen 1984 und 2004 mehrfach erhoben wurden. Hierbei steht die Frage nach der zeitlichen Stabilität des Modells im Vordergrund, denn es ist ungewiss, ob ein Modell, das zu Beginn der 1980er Jahre erfolgreich eingesetzt werden konnte, trotz aller Veränderungen auch weiterhin erklärungskräftig ist. Aber nicht nur im Hinblick auf die Entwicklungen im Zeitverlauf können die Daten betrachtet werden, es kann auch geprüft werden, ob der als allgemeines Modell formulierte Ansatz auf verschiedene Einwanderergruppen anwendbar ist. Unter diesem Gesichtspunkt erscheint es sinnvoll, zum einen nach Nationalitäten zu differenzieren, die sich in Bezug auf die ihnen offenstehenden Handlungsoptionen hin unterscheiden, und zum anderen nach Generationen, da auch zwischen der ersten und den nachfolgenden Generationen erhebliche Unterschiede in den Möglichkeiten zur Sozialintegration gegeben sein dürften. 
Sofern das Modell an den Daten bestätigt werden kann, wird im daran anschließenden Schritt das Erklärungsmodell zu spezifizieren sein und darauf aufbauend mittels des Prozessmodells die Veränderungen im Zeitverlauf inklusive der Variable zu den Einbürgerungs- und Abwanderungsvorgängen geprüft. Vor dem eigentlichen Theorietest sollen jedoch zunächst noch die Indikatoren näher beleuchtet werden, um auch einen Eindruck der gesellschaftlichen Veränderungen im Zusammenhang von Immigration und Integration zu vermitteln, wie sie sich in den SOEP-Daten widerspiegeln. Gleichzeitig müssten sich die Beziehungen der erklärenden und der abhängigen Variablen in den deskriptiven Analysen bereits abzeichnen.

Insgesamt sollen die in diesem Kapitel dargestellten Analysen ein umfassendes Bild der Zusammenhänge des Niveaus der Sozialintegration mit den Naturalisierungen und Abwanderungen ergeben, dabei dürften insbesondere die kausalen Erklärungsmodelle Aufschluss über die strukturellen Zusammenhänge geben.

\subsection{Sozialintegration der Personen mit Migrationshinter- grund in den Daten des Sozio-oekonomischen Panels}

In den amtlichen Statistiken werden seit 1970 verstärkt Daten über die ausländische Bevölkerung ausgewiesen (vgl. Statistisches Bundesamt 1997), in sozialwissenschaftlichen Untersuchungen wurden Personengruppen mit nicht-deutschem Migrationshintergrund in den 1970er und 1980er Jahren immer stärker berücksichtigt. ${ }^{48}$ In den Daten des Sozio-oekonomischen Panels wurden dazu in der Stichprobe $B$ im Verhältnis zu den Haushalten der Stichprobe A gesondert und überproportional Haushalte mit griechischem, italienischem, jugoslawischem, spanischem oder türkischem Haushaltsvorstand erfasst. Neben dieser sogenannten Ausländerstichprobe finden sich in allen anderen Teilstichproben ebenfalls Personen mit ausländischer Staatsangehörigkeit. ${ }^{49} \mathrm{Im}$ Unterschied zu den amtlichen Statistiken stellt

48 Neben einer Vielzahl regionaler und bundesweiter Surveys sind hier vor allem die seit 1970 nahezu ununterbrochenen jährlichen Ausländerbefragungen des Forschungsinstituts MARPLAN und das SOEP (seit 1984) zu nennen. Die Allgemeine Bevölkerungsumfrage der Sozialwissenschaften (ALLBUS) berücksichtigt erst seit 1991 die nicht-deutsche Wohnbevölkerung.

49 Allerdings bleibt unklar, welche Auswahlregeln die Interviewer anwendeten, da in den frühen Erhebungswellen (1984-1995) die speziellen Ausländerfragen nur an Ausländer gestellt wurden. Zu den 
das SOEP nur bedingt ein Abbild der ausländischen Grundgesamtheit dar, bietet aber dafür Zugriff auf weitere Variablen und vermittelt einen Eindruck von den individuellen Entwicklungsprozessen, daher soll die Entwicklung der Sozialintegration in diesem Abschnitt anhand der SOEP-Daten näher untersucht werden.

Die Betrachtung der einzelnen Dimensionen der Sozialintegration soll getrennt nach Herkunftsgruppen und Einwanderergenerationen erfolgen. Um eine ausreichende Fallzahl gewährleisten zu können und mit Rücksicht auf den Umfang, werden im Folgenden nicht alle Nationalitäten einzeln aufgeführt, sondern zum Teil zusammengefasst dargestellt. Berücksichtigung finden dabei Personen, die aus Staaten der EG bzw. EU, aus dem ehemaligen Jugoslawien und der Türkei stammen. Dieser Kreis an Personen ausländischer Herkunft umfasst alle größeren Immigrantengruppen, die im Zuge der Gastarbeiteranwerbung in die Bundesrepublik Deutschland gekommen sind. Zur Analyse der Nationalitätsunterschiede werden neben Personen türkischer und (ex-)jugoslawischer Herkunft die EG/EU-Bürger zu zwei Gruppen zusammengefasst. Befragte mit griechischer, italienischer, portugiesischer und spanischer Staatsangehörigkeit werden als EU-Arbeitsmigranten ${ }^{50}$ und SOEP-Teilnehmer aus den übrigen EU-Staaten ${ }^{51}$ in die Gruppe der sonstigen EUBürger eingeordnet. Die Aufteilung der EU-Bürger in zwei separate Gruppen wird hier vorgenommen, weil davon auszugehen ist, dass zwischen Arbeitsmigranten aus Ländern, mit denen die Bundesrepublik Anwerbeverträge geschlossen hatte und den übrigen EU-Immigranten sozio-strukturelle Unterschiede bestehen. ${ }^{52}$

Um einen Eindruck vom Umfang der jeweiligen Gruppe zu vermitteln, ist in Abbildung 6.1a die Entwicklung der Fallzahlen nach den einzelnen Gruppen getrennt wiedergegeben. Aus den Zahlen geht hervor, dass sich der Umfang der Gruppe

Ausländerfragen zählt auch die Frage nach der Staatsangehörigkeit, was scheinbar zur Folge hatte, dass Ausländer in der Stichproben A und C nur in seltenen Fällen diese Fragen gestellt bekamen.

50 Spanien und Portugal wurden erst 1986 in die EG aufgenommen. Personen aus diesen Ländern und ihre Nachkommen werden in den Analysen aber auch für den Zeitraum 1984-1985 den EU-Arbeitsmigranten zugeordnet, damit eine durchgängig einheitliche Gruppendefinition vorliegt.

51 Die Gruppe der übrigen EU-Staatsangehörigen wurde gemäß der EU-Erweiterung mit dem Datensatz 1995 um Personen finnischer, österreichischer und schwedischer Herkunft erweitert. Diese Erweiterung fällt nicht weiter ins Gewicht, da erst ab 1997 SOEP-Teilnehmer, die nicht zur Ausländerstichprobe $B$ gehören, systematisch die speziellen Fragen für ausländische Befragte gestellt bekamen.

52 Alle anderen Nationalitäten, die im SOEP enthalten sind, wurden nicht gesondert betrachtet, da diese im Sinne der Fragestellung keine einheitliche Gruppe darstellen und getrennt zu geringe Fallzahlen aufweisen. 
klassischer Arbeitsmigranten incl. ihrer Nachkommen im SOEP erheblich reduzierte, während die Gruppe der restlichen EU-Bürger weitgehend stabil geblieben ist. Offensichtlich wird an der Entwicklung, dass sich EU-Arbeitsmigranten und die Befragten aus den übrigen EU-Staaten hinsichtlich ihres Teilnahmeverhaltens am SOEP unterscheiden. Die Zahl türkischer und jugoslawischer Personen in den Stichproben reduzierte sich zwischen 1984 und 2004 um etwas weniger als 50\%, während sich die Zahl der Befragten aus den südlichen EU-Staaten um mehr als zwei Drittel verringerte. ${ }^{53}$

Als Hauptursache für diesen im Vergleich stärkeren Rückgang sind die unter den griechischen, italienischen, portugiesischen und spanischen Befragten höheren durchschnittlichen Abwanderungsraten zu nennen (vgl. Tab. 6.1.2a).

\section{Abbildung 6.1a: Anzahl der Personen in den Herkunftsgruppen nach Jahr der Erhebung}

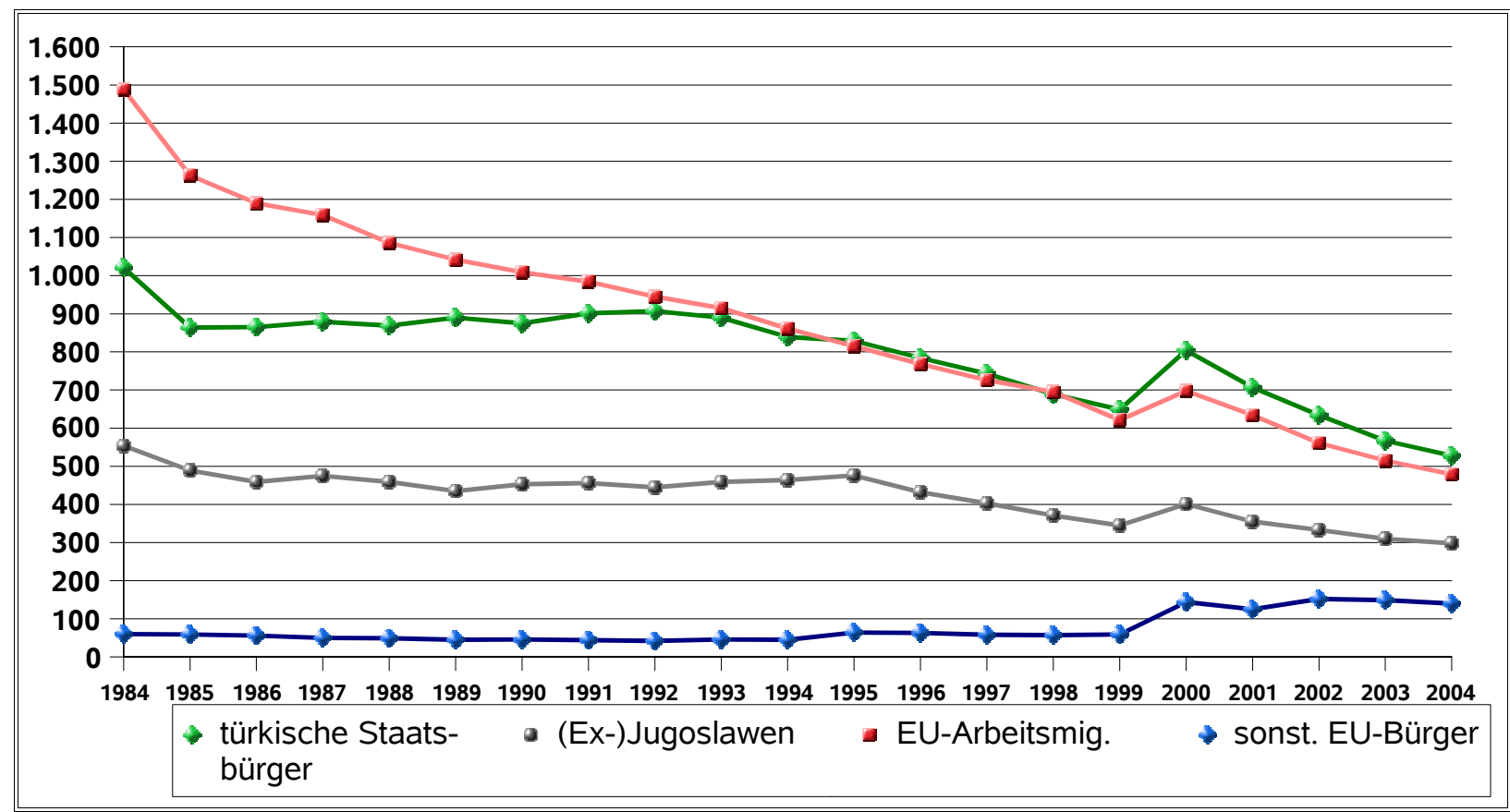

Quelle: SOEP Wellen 1-21

53 Der allgemeine Anstieg von 1999 zu 2000 ist auf die Ergänzungsstichprobe F zurückzuführen. 


\subsubsection{Die Entwicklung der Sozialintegration}

Die Betrachtung der Sozialintegration wird im Anschluss an das Erklärungsmodell (vgl. Kap. 4) in die vier Subdimensionen Kulturation, Platzierung, Interaktion und Identifikation unterteilt. Zu diesem Zweck wurden auf Grundlage der ausgewählten Indikatoren Indizes für die einzelnen Wellen des Sozio-oekonomischen Panels berechnet, in deren Erhebungsinstrumenten die entsprechenden Fragen gleichzeitig enthalten waren. Bedingt durch diese Einschränkung reduziert sich die Anzahl der analysierten Jahrgänge auf zehn. ${ }^{54}$ Für die Analysen wurden alle Personen berücksichtigt, die im Verlauf des Sozio-oekonomischen Panels zumindest einmalig eine der entsprechenden Staatsangehörigkeiten angegeben haben. Dadurch werden auch eingebürgerte Personen in die Berechnungen mit einbezogen, um zumindest eine Verzerrung der Sozialintegration durch den Ausschluss dieser meist gut integrierten Gruppe zu vermeiden (vgl. Salentin/Wilkening 2003).

Tabelle 6.1.1a: Entwicklung der subjektiven Deutschkompetenz ausgewählter Migrantengruppen zwischen 1985 und 2003

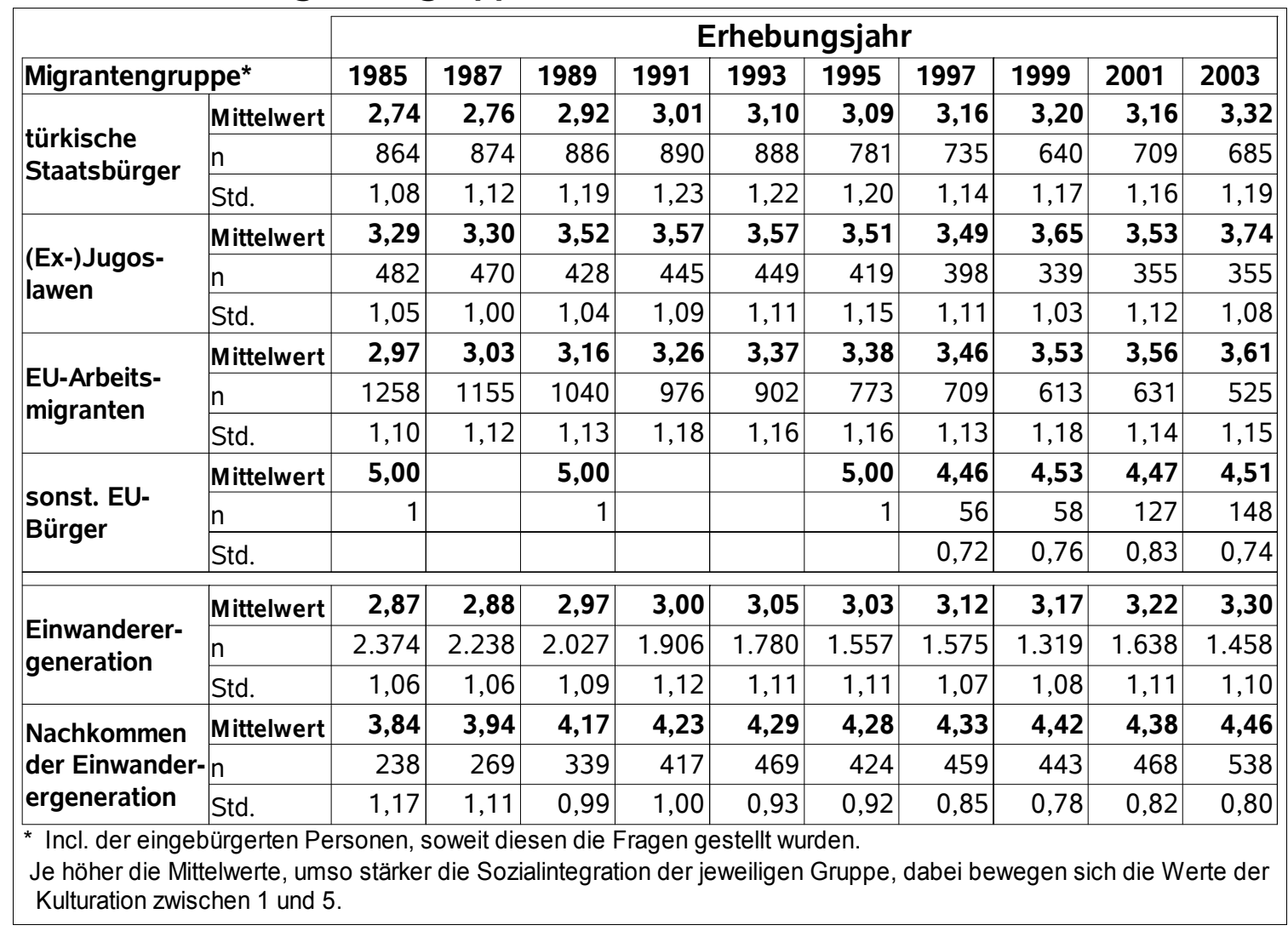

54 Analysiert wurden die Daten der Wellen 1985, 1989, 1991, 1993, 1995, 1997, 1999, 2001 und 2003. 
Um die kulturelle Annäherung an die deutsche Aufnahmegesellschaft im Verlauf des Panels darstellen zu können, wurden Mittelwerte für den Index der subjektiven Bewertung der mündlichen und schriftlichen Deutschkompetenz berechnet, die in Tabelle 6.1.1a wiedergegeben sind. Die Werte vermitteln ein Bild, das fast durchgängig von einem Zuwachs der subjektiven Deutschkompetenz gekennzeichnet ist. Nur für die Gruppe der sonstigen EU-Bürger kann dieser Befund nicht bestätigt werden, was aber möglicherweise mit der schon angesprochenen Erhebungspraxis in Zusammenhang gesehen werden muss, da erst ab 1997 eine kontinuierliche Erfassung stattgefunden hat. Im Vergleich weisen unter den herkunftsbezogenen Gruppen die türkischen Befragten die niedrigsten Werte auf, gefolgt von den EU-Arbeitsmigranten und den (Ex-)Jugoslawen. Die Gruppe der übrigen EU-Bürger bewertet mit Werten um 4,5 bei gleichzeitig geringer Standardabweichung die eigenen Deutschkenntnisse durchschnittlich am Besten. Was aber nicht weiter überraschend ist, da österreichische Staatsbürger rund ein Drittel dieser Gruppe stellen. Ohne die österreichischen Befragten liegen die Mittelwerte etwas niedriger, aber immer noch höher als bei den übrigen Nationalitätsgruppen $(4,11 ; 4,30 ; 4,24$; 4,25). ${ }^{55}$ Werden die Angaben getrennt nach Immigrantengeneration und den nachfolgenden Generationen ausgewertet, so ergibt sich auch für diese beiden Gruppen ein durchgängiger Anstieg der subjektiven Deutschkenntnisse. Allerdings erreicht die Gruppe der Immigranten nicht einmal das Ausgangsniveau der nachfolgenden Generationen, was für die Bedeutung der primären Kulturation spricht. Dieses Ergebnis bestätigt internationale Studien zum Spracherwerb von Immigranten und den folgenden Generationen (vgl. Lopez 1999; Protes/Rumbaut 2001; Alba 2004).

Für die Analyse der Platzierung auf Basis des Erwerbseinkommens und der beruflichen Arbeitsautonomie standen zwar Informationen für alle Jahrgänge zur Verfügung, allerdings wurde die Auswertung aus Gründen der Vergleichbarkeit auf dieselben zehn Jahrgänge beschränkt (Tab. 6.1.1b). Die Sozialintegration über den Arbeitsmarkt weist im Vergleich zur Kulturation ein deutlich anderes Muster auf. Die Werte bewegen sich von 1985 bis 1987 nur geringfügig, um dann 1991 anzustei-

55 Bei der Interpretation der Werte müssen die unterschiedlichen Sprunganweisungen in den einzelnen Erhebungsinstrumenten berücksichtigt werden, die zu einer selektiven (1997-2001) und einer vollständigen (2003) Erfassung der Sprachkompetenz und Identifikation bei eingebürgerten Respondenten führte (vgl. Tab. 5.1.2.1a). Die dadurch möglicherweise hervorgerufenen Verzerrungen sind nicht abschätzbar und müssen daher bei der Interpretation der Ergebnisse Berücksichtigung werden. 
gen, was für eine bessere Einbindung in den Arbeitsmarkt spricht. In der Folgezeit sinken die Platzierungswerte leicht unter dieses Niveau ab, wenn auch zum Teil danach eine gewisse Erholung zu verzeichnen ist, liegen die Werte 2003 fast durchgängig unter denen von 1991. Dieses Muster steht in direktem Zusammenhang mit den Arbeitslosenzahlen und das Maximum von 1991 gibt die durch den Vereinigungsboom niedrige Arbeitslosenrate wieder (vgl. Institut der deutschen Wirtschaft 2001; ebd. 2005). Die höchsten Werte weisen hierbei wiederum die sonstigen EUBürger und die niedrigsten die türkischen Staatsbürger auf. Mit tendenziell ähnlichen Durchschnittswerten rangieren dazwischen die (Ex-)Jugoslawen und EU-Arbeitsmigranten.

Tabelle 6.1.1b: Entwicklung der Sozialintegration über den Arbeitsmarkt nach ausgewäh/ten Migrantengruppen zwischen 1985 und 2003

\begin{tabular}{|c|c|c|c|c|c|c|c|c|c|c|c|}
\hline \multirow{2}{*}{\multicolumn{2}{|c|}{ Migrantengruppe* }} & \multicolumn{10}{|c|}{ Erhebungsjahr } \\
\hline & & 1985 & 1987 & 1989 & 1991 & 1993 & 1995 & 1997 & 1999 & 2001 & 2003 \\
\hline \multirow{3}{*}{$\begin{array}{l}\text { türkische } \\
\text { Staatsbürger }\end{array}$} & Mittelwert & 2,42 & 2,49 & 2,61 & 2,92 & 2,64 & 2,50 & 2,55 & 2,52 & 2,56 & 2,50 \\
\hline & $n$ & 589 & 605 & 557 & 599 & 584 & 528 & 467 & 423 & 501 & 419 \\
\hline & Std. & 1,40 & 1,36 & 1,21 & 1,35 & 1,47 & 1,57 & 1,59 & 1,56 & 1,63 & 1,65 \\
\hline \multirow{3}{*}{\begin{tabular}{|l|} 
(Ex-)Jugos- \\
lawen
\end{tabular}} & Mittelwert & 2,95 & 2,93 & 2,85 & 3,15 & 2,97 & 2,79 & 2,63 & 2,81 & 2,97 & 2,82 \\
\hline & $n$ & 392 & 383 & 342 & 364 & 362 & 368 & 346 & 269 & 279 & 245 \\
\hline & Std. & 1,39 & 1,38 & 1,34 & 1,35 & 1,36 & 1,50 & 1,59 & 1,49 & 1,41 & 1,54 \\
\hline \multirow{3}{*}{$\begin{array}{l}\text { EU-Arbeits- } \\
\text { migranten }\end{array}$} & Mittelwert & 2,90 & 2,84 & 2,91 & 3,21 & 2,96 & 2,91 & 2,90 & 3,02 & 3,10 & 3,07 \\
\hline & $n$ & 957 & 881 & 776 & 741 & 684 & 594 & 526 & 446 & 449 & 372 \\
\hline & Std. & 1,22 & 1,28 & 1,24 & 1,34 & 1,47 & 1,50 & 1,61 & 1,51 & 1,47 & 1,48 \\
\hline \multirow{3}{*}{$\begin{array}{l}\text { sonst. EU- } \\
\text { Bürger }\end{array}$} & Mittelwert & 3,65 & 3,73 & 3,86 & 3,85 & 3,58 & 3,89 & 3,72 & 3,81 & 3,78 & 3,75 \\
\hline & $n$ & 49 & 42 & 36 & 42 & 40 & 52 & 49 & 42 & 90 & 95 \\
\hline & Std. & 1,41 & 1,41 & 1,38 & 1,56 & 1,85 & 1,63 & 1,66 & 1,62 & 1,63 & 1,66 \\
\hline \multirow{3}{*}{\begin{tabular}{|l|} 
Einwanderer- \\
generation
\end{tabular}} & Mittelwert & 2,81 & 2,80 & 2,88 & 3,22 & 2,97 & 2,83 & 2,77 & 2,85 & 2,92 & 2,79 \\
\hline & n & 1.829 & 1.734 & 1.502 & 1.481 & 1.366 & 1.302 & 1.119 & 912 & 1.121 & 913 \\
\hline & Std. & 1,34 & 1,34 & 1,26 & 1,32 & 1,44 & 1,55 & 1,63 & 1,54 & 1,56 & 1,56 \\
\hline \multirow{3}{*}{$\begin{array}{l}\text { Nachkommen } \\
\text { der Einwande } \\
\text { generation }\end{array}$} & Mittelwert & 2,53 & 2,48 & 2,51 & 2,67 & 2,47 & 2,52 & 2,61 & 2,70 & 2,79 & 2,98 \\
\hline & & 191 & 210 & 237 & 310 & 347 & 342 & 354 & 344 & 380 & 390 \\
\hline & Std. & 1,39 & 1,42 & 1,32 & 1,46 & 1,57 & 1,59 & 1,63 & 1,61 & 1,64 & 1,71 \\
\hline
\end{tabular}

Quelle: SOEP Wellen 2, 4, 6, 8, 10, 12, 14, 16, 18 und 20 
Die Mittelwerte der Einwanderergeneration bilden die generelle Entwicklung ab, im Falle der nachfolgenden Generationen zeigt sich jedoch eine andere Tendenz. Zwischen 1985-1989 sind in der relativ kleinen Gruppe im Durchschnitt relativ niedrige Positionierungen zu verzeichnen, gefolgt von einem lokalen Hoch im Jahre 1991. Von dem niedrigeren Niveau 1993 ausgehend beginnen dann aber die Werte stetig zu steigen und erreichen 2003 mit 2,98 einen Wert, der erheblich über dem Ausgangsniveau Mitte der 1980er Jahre liegt und der auch den Durchschnittswert der Einwanderergeneration erstmals übertrifft, wobei die etwas höhere Standardabweichung etwas heterogenere Verhältnisse zum Ausdruck bringt. Die zu Beginn des Panels höheren Werte der ersten Generation bleiben im Verlauf relativ konstant und können als Indiz für eine konstante Positionierung auf dem Arbeitsmarkt gewertet werden. Im Falle der nachfolgenden Generationen spricht das stetige Wachstum für eine zunehmende Sozialintegration über den Arbeitsmarkt. Ein Grund für die bessere Positionierung der zweiten und dritten Generation dürfte neben der besseren Kulturation auch in den durchschnittlich höheren Bildungsabschlüssen der jüngeren Generationen zu sehen sein (Esser 2001, 52). Insgesamt unterscheiden sich jedoch die betrachteten Nationalitätengruppen sehr viel stärker in ihrer Positionierung auf dem Arbeitsmarkt als die Migrantengenerationen, insbesondere die im Vergleich deutlich schlechteren Werte der türkischen Staatsbürger bestätigen die Ergebnisse anderer Untersuchungen (vgl. Esser 2001, 52-56; Rheinisch-Westfälisches Institut für Wirtschaftsforschung e.V. 2001, 35-39; Kalter/Granato 2004).

Bezogen auf die Interaktion ergibt sich, allerdings auf höherem Niveau, zwischen den Gruppen ein ähnliches Bild wie bei der Kulturation. Wie in Tabelle 6.1.1c zu erkennen ist, weisen die Personen türkischer Herkunft durchgängig die niedrigsten Besuchswerte auf, während die eher kleine Gruppe der sonstigen EU-Bürger im Zeitraum von 1997 bis 2003 dem Maximalwert von 1 nahe kommt und zum Teil sogar erreicht. Auch hier liegen die Werte für Personen aus Ex-Jugoslawien und den EU-Anwerbeländern wieder zwischen denen der anderen Gruppen. Wie im Falle der Kulturation weisen die Türken, die in der Bundesrepublik die größte Immigrantencommunity bilden, durchschnittlich die niedrigsten Werte auf.

Wird die Community im Sinne einer ethnischen Gelegenheitsstruktur interpretiert, so ist es nicht weiter verwunderlich, dass bei gegebener Segregation Immigranten- 
gruppen mit vollständigen Communities im Durchschnitt weniger Besuche von Deutschen erhalten und auch umgekehrt weniger Deutsche besuchen. Insofern passen die geringeren Werte der ersten und die höheren der nachfolgenden Generation ebenfalls in dieses Bild, da über Kindergarten, Schule und Ausbildung institutionell geprägte Gelegenheitsstrukturen existieren. Die Schwankung der Werte zwischen den einzelnen Erhebungszeitpunkten innerhalb der jeweiligen Gruppen sollen hier nicht weiter interpretiert werden, da nicht abzuschätzen ist, was Ausfallprozesse und Änderungen der Erhebungsinstrumente zu diesen beigetragen haben können.

Tabelle 6.1.1c: Entwicklung der Sozialintegration ausgewäh/ter Migrantengruppen über soziale Kontakte zu Deutschen zwischen 1985 und 2003

\begin{tabular}{|c|c|c|c|c|c|c|c|c|c|c|c|}
\hline & & \multicolumn{10}{|c|}{ Erhebungsjahr } \\
\hline \multicolumn{2}{|c|}{ Migrantengruppe* } & 1985 & 1987 & 1989 & 1991 & 1993 & 1995 & 1997 & 1999 & 2001 & 2003 \\
\hline \multirow{3}{*}{$\begin{array}{l}\text { türkische } \\
\text { Staatsbürger }\end{array}$} & Mittelwert & 0,69 & 0,73 & 0,70 & 0,69 & 0,71 & 0,73 & 0,75 & 0,79 & 0,72 & 0,72 \\
\hline & n & 862 & 873 & 885 & 890 & 888 & 782 & 738 & 627 & 694 & 561 \\
\hline & Std. & 0,42 & 0,41 & 0,43 & 0,43 & 0,41 & 0,41 & 0,40 & 0,38 & 0,41 & 0,42 \\
\hline \multirow{3}{*}{$\begin{array}{l}\text { (Ex-)Jugos- } \\
\text { lawen }\end{array}$} & Mittelwert & 0,85 & 0,87 & 0,89 & 0,90 & 0,84 & 0,84 & 0,83 & 0,86 & 0,84 & 0,85 \\
\hline & $n$ & 482 & 470 & 428 & 445 & 448 & 420 & 398 & 333 & 350 & 313 \\
\hline & Std. & 0,33 & 0,32 & 0,29 & 0,28 & 0,35 & 0,35 & 0,34 & 0,33 & 0,35 & 0,34 \\
\hline \multirow{3}{*}{$\begin{array}{l}\text { EU-Arbeits- } \\
\text { migranten }\end{array}$} & Mittelwert & 0,78 & 0,81 & 0,82 & 0,79 & 0,81 & 0,81 & 0,83 & 0,82 & 0,85 & 0,86 \\
\hline & $n$ & 1.254 & 1.155 & 1.039 & 974 & 902 & 772 & 710 & 611 & 629 & 513 \\
\hline & Std. & 0,39 & 0,36 & 0,36 & 0,38 & 0,37 & 0,36 & 0,35 & 0,36 & 0,32 & 0,31 \\
\hline \multirow{3}{*}{$\begin{array}{l}\text { sonst. EU- } \\
\text { Bürger }\end{array}$} & Mittelwert & 1,00 & & 1,00 & & & 1,00 & 1,00 & 0,98 & 0,98 & 0,98 \\
\hline & $n$ & 1 & & 1 & & & 1 & 55 & 58 & 125 & 146 \\
\hline & Std. & & & & & & & 0,00 & 0,13 & 0,12 & 0,12 \\
\hline & & & & & & & & & & & \\
\hline \multirow{3}{*}{$\begin{array}{l}\text { Einwanderer- } \\
\text { generation }\end{array}$} & Mittelwert & 0,75 & 0,78 & 0,77 & 0,75 & 0,75 & 0,75 & 0,77 & 0,79 & 0,79 & 0,79 \\
\hline & $n$ & 2.368 & 2.237 & 2.027 & 1.904 & 1.780 & 1.558 & 1.580 & 1.282 & 1.576 & 1.273 \\
\hline & Std. & 0,40 & 0,39 & 0,40 & 0,41 & 0,41 & 0,40 & 0,39 & 0,38 & 0,38 & 0,38 \\
\hline \multirow{3}{*}{$\begin{array}{l}\text { Nachkommen } \\
\text { der Einwander- } \\
\text { ergeneration }\end{array}$} & Mittelwert & 0,86 & 0,90 & 0,94 & 0,90 & 0,91 & 0,92 & 0,92 & 0,93 & 0,91 & 0,91 \\
\hline & $n$ & 238 & 269 & 338 & 417 & 468 & 424 & 457 & 436 & 466 & 476 \\
\hline & Std. & 0,33 & 0,28 & 0,21 & 0,27 & 0,26 & 0,23 & 0,23 & 0,22 & 0,27 & 0,26 \\
\hline
\end{tabular}

Quelle: SOEP Wellen 2, 4, 6, 8, 10, 12, 14, 16, 18 und 20 
Die Werte zur emotionalen Identifikation mit den Deutschen bei gleichzeitiger gefühlter Distanz zur Herkunftsgemeinschaft sind zu Beginn des Panels für alle Gruppen am niedrigsten ausgeprägt und steigen dann im Verlauf der Jahre relativ kontinuierlich an (vgl. Tab. 6.1.1d). Nur die Gruppe der sonstigen EU-Bürger weicht auf hohem Niveau hiervon ab, wobei die geringfügig höheren Standardabweichungen auf etwas heterogenere Antworten hindeuten. Mit der Ausweitung der Stichprobe nach 1999 sinken die Werte unter den Befragten dieser Gruppe leicht ab. Insgesamt fällt die Identifikation mit den Deutschen in der Teilgruppe der sonstigen EUBürger trotzdem am stärksten aus, während die EU-Arbeitsmigranten zwischen den

Tabelle 6.1.1d: Entwicklung der Sozialintegration ausgewäh/ter Migrantengruppen über die emotionale Identifikation mit der Aufnahmegesellschaft zwischen 1985 und 2003

\begin{tabular}{|c|c|c|c|c|c|c|c|c|c|c|c|}
\hline \multirow[b]{2}{*}{ Migrantengruppe* } & & \multicolumn{10}{|c|}{ Erhebungsjahr } \\
\hline & & 1985 & 1987 & 1989 & 1991 & 1993 & 1995 & 1997 & 1999 & 2001 & 2003 \\
\hline \multirow{3}{*}{$\begin{array}{l}\text { türkische } \\
\text { Staatsbürger }\end{array}$} & Mittelwert & 1,66 & 1,73 & 1,77 & 1,83 & 1,85 & 1,86 & 2,22 & 2,37 & 2,46 & 2,50 \\
\hline & $n$ & 858 & 873 & 887 & 885 & 881 & 779 & 731 & 640 & 708 & 683 \\
\hline & Std. & 0,85 & 0,94 & 0,91 & 0,96 & 0,98 & 0,99 & 0,92 & 0,90 & 0,88 & 0,89 \\
\hline \multirow{3}{*}{ (Ex-)Jugoslawen } & Mittelwert & 2,17 & 2,20 & 2,40 & 2,57 & 2,58 & 2,51 & 2,44 & 2,60 & 2,73 & 2,87 \\
\hline & $n$ & 482 & 470 & 428 & 445 & 448 & 421 & 402 & 339 & 355 & 355 \\
\hline & Std. & 1,09 & 1,01 & 1,10 & 1,07 & 1,11 & 1,11 & 0,93 & 0,87 & 0,89 & 0,92 \\
\hline \multirow{3}{*}{$\begin{array}{l}\text { EU-Arbeits- } \\
\text { migranten }\end{array}$} & Mittelwert & 1,86 & 2,01 & 2,09 & 2,06 & 2,12 & 2,13 & 2,28 & 2,30 & 2,57 & 2,59 \\
\hline & $n$ & 1.255 & 1.153 & 1.040 & 970 & 899 & 770 & 711 & 615 & 629 & 523 \\
\hline & Std. & 1,00 & 1,06 & 1,04 & 1,05 & 1,03 & 1,03 & 0,97 & 0,94 & 0,87 & 0,87 \\
\hline \multirow{3}{*}{ sonst. EU-Bürger } & Mittelwert & 4,50 & & 4,50 & & & 1,50 & 2,86 & 3,03 & 2,94 & 2,91 \\
\hline & $n$ & 1 & & 1 & & & 1 & 55 & 58 & 127 & 150 \\
\hline & Std. & & & & & & & 1,13 & 1,26 & 1,08 & 1,10 \\
\hline \multirow{3}{*}{$\begin{array}{l}\text { Einwanderer- } \\
\text { generation }\end{array}$} & Mittelwert & 1,79 & 1,86 & 1,92 & 1,96 & 1,96 & 1,97 & 2,20 & 2,29 & 2,48 & 2,55 \\
\hline & n & 2.365 & 2.235 & 2.028 & 1.896 & 1.772 & 1.553 & 1.576 & 1.321 & 1.638 & 1.458 \\
\hline & Std. & 0,94 & 0,96 & 0,97 & 1,00 & 0,98 & 1,00 & 0,92 & 0,91 & 0,88 & 0,90 \\
\hline \multirow{3}{*}{$\begin{array}{l}\text { Nachkommen } \\
\text { der Einwanderer- } \\
\text { generation }\end{array}$} & Mittelwert & 2,46 & 2,69 & 2,68 & 2,59 & 2,65 & 2,61 & 2,76 & 2,87 & 3,00 & 2,95 \\
\hline & n & 238 & 268 & 339 & 416 & 466 & 425 & 458 & 443 & 466 & 536 \\
\hline & Std. & 1,24 & 1,22 & 1,14 & 1,15 & 1,16 & 1,15 & 1,02 & 0,92 & 0,90 & 0,96 \\
\hline
\end{tabular}

Quelle: SOEP Wellen 2, 4, 6, 8, 10, 12, 14, 16, 18 und 20 
Personen türkischer und (ex-)jugoslawischer Herkunft rangieren. Aber nicht erst seit dem Zusammenbruch des ethnisch heterogenen jugoslawischen Bundesstaates, sondern auch in der Zeit vor 1991 ist die emotionale Identifikation der jugoslawischen Befragten mit den Deutschen am stärksten bzw. mit Berücksichtigung der sonstigen Ausländer im SOEP am zweit stärksten ausgeprägt. Auch die Aufteilung nach Generationen spiegelt den tendenziellen Anstieg der Identifikation mit den Deutschen wider, wobei die jüngeren Generationen wie zuvor deutlich höhere Werte aufweisen als die erste Immigrantengeneration.

\subsubsection{Sozialintegration im Verhältnis zu den Abwanderungs- und Einbürgerungsvorgängen}

Im vorhergehenden Abschnitt konnte anhand der SOEP-Daten gezeigt werden, dass die Sozialintegration der Immigranten und ihrer Nachkommen sich in den letzten Jahrzehnten deutlich verbessert hat. Entsprechend des dargestellten Erklärungsmodells sollte die Sozialintegration der Bevölkerung mit Migrationshintergrund einen Effekt auf die Abwanderungs- und Einbürgerungsvorgänge ausüben. Geringe Einbindung in die Aufnahmegesellschaft sollte tendenziell eher zu Abwanderung und stärkere Integration eher mit Einbürgerung in Zusammenhang stehen. Bedingt durch die mehrmaligen Veränderungen der rechtlichen Rahmenbedingungen und spezieller aufenthaltsrechtlicher Bestimmungen für Ausländer wäre es nicht korrekt, die Zahl der Akte als unmittelbaren Ausdruck der sozialen Integration zu werten.

Insgesamt lassen sich 1.138 Abwanderungen und 393 Einbürgerungen für Personen nicht-deutscher Herkunft in den Daten des Sozio-oekonomischen Panels finden. Aufgeteilt nach Nationalitätsgruppen und Generationen sind einige Unterschiede zu erkennen (vgl. Tab. 6.1.2a). Überraschenderweise ist es die Gruppe der relativ gut integrierten Befragten aus EU-Anwerbeländern, die mit rund $27 \%$ eine erheblich höhere Abwanderungsrate als alle anderen Gruppen aufweist. Mit deutlichem Abstand folgen die Personen mit (ex-)jugoslawischer Herkunft (15,33\%) und die anderen Gruppen mit Werten knapp über $12 \%$. 
Tabelle 6.1.2a: Umfang der Abwanderungs- und Einbürgerungsvorgänge ausgewählter Migrantengruppen zwischen 1985 und 2004

\begin{tabular}{|c|c|c|c|c|c|}
\hline \multirow{2}{*}{\multicolumn{2}{|c|}{ Migrantengruppe }} & \multicolumn{2}{|c|}{ Abwanderung } & \multicolumn{2}{|c|}{ Einbürgerung } \\
\hline & & \multirow{2}{*}{\begin{tabular}{|l|} 
ja \\
257
\end{tabular}} & \multirow{2}{*}{\begin{tabular}{|l|} 
nein \\
1.803
\end{tabular}} & \multirow{2}{*}{\begin{tabular}{|l|}
$j a$ \\
185
\end{tabular}} & \multirow{2}{*}{$\begin{array}{c}\text { nein } \\
1.875\end{array}$} \\
\hline \multirow{2}{*}{ türkische Herkunft } & $n$ & & & & \\
\hline & $\%$ & 12,48 & 87,52 & 8,98 & 91,02 \\
\hline \multirow{2}{*}{$\begin{array}{l}\text { (ex-) jugoslawische } \\
\text { Herkunft }\end{array}$} & $n$ & 159 & 878 & 80 & 957 \\
\hline & $\%$ & 15,33 & 84,67 & 7,71 & 92,29 \\
\hline \multirow{2}{*}{ EU-Arbeitsmigranter } & $n$ & 612 & 1.647 & 20 & 2.239 \\
\hline & $\%$ & 27,09 & 72,91 & 0,89 & 99,11 \\
\hline \multirow{2}{*}{ sonst. EU-Bürger } & $n$ & 34 & 245 & 8 & 271 \\
\hline & $\%$ & 12,19 & 87,81 & 2,87 & 97,13 \\
\hline \multirow{2}{*}{$\begin{array}{l}\text { sonst. Personen } \\
\text { nicht-deutscher } \\
\text { Herkunft }\end{array}$} & n & 76 & 518 & 100 & 494 \\
\hline & $\%$ & 12,79 & 87,21 & 16,83 & 83,17 \\
\hline \multirow{2}{*}{$\begin{array}{l}\text { Einwanderer- } \\
\text { generation }\end{array}$} & $\mathrm{n}$ & 953 & 3.635 & 275 & 4.313 \\
\hline & $\%$ & 20,77 & 79,23 & 5,99 & 94,01 \\
\hline \multirow{2}{*}{$\begin{array}{l}\text { Nachkommen } \\
\text { der Einwanderer- } \\
\text { generation }\end{array}$} & $n$ & 185 & 1.456 & 118 & 1.523 \\
\hline & $\%$ & 11,27 & 88,73 & 7,19 & 92,81 \\
\hline
\end{tabular}

Quelle: SOEP Wellen 1-21

Der Vergleich zwischen den Migrantengenerationen fällt gemäß der theoretischen Erwartungen aus, von den Angehörigen der Immigrantengeneration wandern relativ gesehen mit $20,77 \%$ fast doppelt so viele Personen ab wie von den nachfolgenden Generationen (11,27\%). Auch im Hinblick auf die Einbürgerung sind Unterschiede zwischen den einzelnen Nationalitätsgruppen erkennbar, die nicht als mit den theoretischen Erwartungen übereinstimmend interpretiert werden können. Es sind insbesondere die Personen türkischer und (ex-)jugoslawischer Herkunft, die trotz der geringeren Sozialintegration sich häufiger einbürgern lassen. Für die besser oder zumindest gleich gut integrierten EU-Bürger liegen die Einbürgerungsanteile mit knapp unter $1 \%$ für die Befragten aus EU-Anwerbeländern und mit 2,87\% für die sonstigen EU-Bürger erheblich niedriger. Die insgesamt höchsten Einbürgrungswerte weist die sehr heterogene Restgruppe der sonstigen Personen nicht-deutscher Herkunft mit $16,83 \%$ auf. ${ }^{56}$

56 Die heterogene Zusammensetzung dieser Gruppe und die kleinen bis sehr kleinen Fallzahlen der einzelnen Nationalitäten lassen eine differenziertere Analyse als nicht sinnvoll erscheinen. 
Allerdings bestätigen die starken Unterschiede zwischen den einzelnen Nationalitäten insgesamt die nationalen und internationalen Forschungsergebnisse zur Naturalisierung von Einwanderern (vgl. Kap. 3.2.2). Für die Bundesrepublik ist dabei charakteristisch, dass sich Personen aus EU-Staaten im Verhältnis sehr viel seltener einbürgern lassen als Personen, die nicht aus der EU stammen. Der Hauptgrund für diesen Unterschied muss wohl in den aufenthaltsrechtlichen Bestimmungen gesehen werden, die Bürgern der EU ein weitgehendes Niederlassungsrecht innerhalb der Bundesrepublik zugestehen. Für diese Gruppe entfällt somit der Anreiz durch die Einbürgerung den Aufenthaltsstatus zu verfestigen, während Personen aus Drittstaaten erst mit der Einbürgerung vor Abschiebungen geschützt sind. Die Einbürgerungsraten der Migrantengenerationen hingegen fallen ebenso wie die Abwanderungsraten, wenn auch weniger deutlich, theoriekonform aus. Die besser integrierten Nachkommen der Einwanderergeneration wurden demnach häufiger eingebürgert als die Angehörigen der ersten Generation.

Im Folgenden soll nun die Sozialintegration der abgewanderten bzw. eingebürgerten Respondenten im Verhältnis zu den Inländern ohne Änderung der Staatsangehörigkeit betrachtet werden. Dazu wurden für die genannten drei Gruppen die Mittelwerte der Sozialintegration getrennt nach den einzelnen Subdimensionen berechnet. Den theoretischen Erwartungen zufolge sollten die später abgewanderten Befragten die geringste und die im Verlauf der Jahre irgendwann eingebürgerten Befragten die höchste Sozialintegration aufweisen. Für Personen, die bis 2004 nicht abwanderten und auch nicht eingebürgert wurden, ist dem Erklärungsmodell gemäß mit Werten zwischen denen der beiden anderen Gruppen zu zurechnen.

Aus Tabelle 6.1.2b ist zu erkennen, dass die über alle Gruppen hinweg tendenziell ansteigenden Werte der Kulturation erwartungsgemäß ausgeprägt sind. Bereits 1985 weisen die Abwanderer die geringsten subjektiven Deutschkenntnisse auf gefolgt von den Personen ohne Änderungen. Die höchsten Durschnittswerte für die sprachliche Integration ergeben sich für die im Verlauf des Panels eingebürgerten Befragten. Entsprechend der getroffenen Gruppendefinition gehen dabei die Fallzahlen der Abwanderer rapide zurück, während die der anderen Gruppen eher als konstant bezeichnet werden können. Der Anstieg der Fallzahlen von 2001 zu 2003 
Tabelle 6.1.2b: Entwicklung der Sozialintegration von später abgewanderten bzw. eingebürgerten Immigranten im Kontrast zu befragten Inländern ohne deutsche Staatsangehörigkeit von 1985 bis 2003

\begin{tabular}{|c|c|c|c|c|c|c|c|c|c|c|c|}
\hline & & \multicolumn{10}{|c|}{ Kulturation } \\
\hline & & 1985 & 1987 & 1989 & 1991 & 1993 & 1995 & 1997 & 1999 & 2001 & 2003 \\
\hline \multirow{3}{*}{ Abwanderer } & Mittelwert & 2,69 & 2,72 & 2,81 & 2,88 & 2,83 & 2,85 & 2,91 & 2,96 & 3,16 & 3,25 \\
\hline & $n$ & 596 & 527 & 410 & 348 & 313 & 228 & 213 & 137 & 84 & 42 \\
\hline & Std. & 1,01 & 1,06 & 1,07 & 1,11 & 1,08 & 1,10 & 1,06 & 1,21 & 1,25 & 1,48 \\
\hline \multirow{3}{*}{$\begin{array}{l}\text { Inländer } \\
\text { ohne deut. } \\
\text { Staatsb. }\end{array}$} & Mittelwert & 3,00 & 3,03 & 3,17 & 3,24 & 3,34 & 3,31 & 3,42 & 3,50 & 3,48 & 3,57 \\
\hline & $n$ & 1.894 & 1.854 & 1.805 & 1.811 & 1.765 & 1.585 & 1.623 & 1.477 & 1.860 & 1.680 \\
\hline & Std. & 1,10 & 1,11 & 1,16 & 1,20 & 1,18 & 1,18 & 1,15 & 1,15 & 1,16 & 1,16 \\
\hline \multirow{3}{*}{$\begin{array}{l}\text { Einge- } \\
\text { bürgerte }\end{array}$} & Mittelwert & 3,61 & 3,61 & 3,67 & 3,76 & 3,79 & 3,74 & 3,73 & 3,84 & 3,70 & 3,91 \\
\hline & $n$ & 119 & 122 & 144 & 160 & 165 & 162 & 174 & 135 & 150 & 262 \\
\hline & Std. & 1,13 & 1,05 & 1,13 & 1,13 & 1,18 & 1,12 & 0,98 & 0,92 & 1,00 & 1,00 \\
\hline & & \multicolumn{10}{|c|}{ Platzierung } \\
\hline \multirow{3}{*}{ Abwanderer } & Mittelwert & 2,72 & 2,65 & 2,73 & 2,91 & 2,63 & 2,41 & 2,28 & 2,12 & 2,76 & 2,71 \\
\hline & $n$ & 443 & 393 & 274 & 246 & 215 & 183 & 132 & 82 & 46 & 19 \\
\hline & Std. & 1,33 & 1,38 & 1,28 & 1,40 & 1,48 & 1,67 & 1,73 & 1,79 & 1,81 & 1,43 \\
\hline \multirow{3}{*}{$\begin{array}{l}\text { Inländer } \\
\text { ohne deut. } \\
\text { Staatsb. }\end{array}$} & Mittelwert & 2,80 & 2,80 & 2,85 & 3,16 & 2,88 & 2,78 & 2,77 & 2,86 & 2,87 & 2,85 \\
\hline & $n$ & 1.485 & 1.438 & 1.342 & 1.389 & 1.330 & 1.279 & 1.141 & 984 & 1.237 & 1.088 \\
\hline & Std. & 1,34 & 1,34 & 1,27 & 1,35 & 1,49 & 1,55 & 1,62 & 1,54 & 1,58 & 1,61 \\
\hline \multirow{3}{*}{$\begin{array}{c}\text { Einge- } \\
\text { bürgerte }\end{array}$} & Mittelwert & 2,80 & 2,66 & 2,80 & 3,14 & 3,09 & 3,03 & 2,78 & 2,86 & 2,98 & 2,84 \\
\hline & $n$ & 89 & 109 & 117 & 150 & 162 & 175 & 193 & 187 & 215 & 194 \\
\hline & Std. & 1,48 & 1,45 & 1,23 & 1,38 & 1,43 & 1,51 & 1,59 & 1,48 & 1,53 & 1,64 \\
\hline & & \multicolumn{10}{|c|}{ Interaktion } \\
\hline \multirow{3}{*}{ Abwanderer } & Mittelwert & 0,73 & 0,76 & 0,75 & 0,71 & 0,67 & 0,72 & 0,69 & 0,72 & 0,68 & 0,73 \\
\hline & $n$ & 596 & 527 & 410 & 348 & 313 & 228 & 214 & 137 & 84 & 42 \\
\hline & Std. & 0,41 & 0,40 & 0,41 & 0,43 & 0,44 & 0,42 & 0,43 & 0,43 & 0,44 & 0,43 \\
\hline \multirow{3}{*}{$\begin{array}{l}\text { Inländer } \\
\text { ohne deut. } \\
\text { Staatsb. }\end{array}$} & Mittelwert & 0,77 & 0,80 & 0,79 & 0,78 & 0,79 & 0,79 & 0,81 & 0,83 & 0,82 & 0,82 \\
\hline & $n$ & 1.888 & 1.853 & 1.803 & 1.809 & 1.764 & 1.586 & 1.626 & 1.460 & 1.849 & 1.666 \\
\hline & Std. & 0,39 & 0,37 & 0,38 & 0,39 & 0,38 & 0,38 & 0,36 & 0,35 & 0,35 & 0,35 \\
\hline \multirow{3}{*}{$\begin{array}{l}\text { Einge- } \\
\text { bürgerte }\end{array}$} & Mittelwert & 0,86 & 0,88 & 0,89 & 0,88 & 0,89 & 0,87 & 0,88 & 0,91 & 0,85 & 0,92 \\
\hline & $n$ & 119 & 122 & 145 & 160 & 165 & 162 & 173 & 114 & 104 & 37 \\
\hline & Std. & 0,30 & 0,30 & 0,29 & 0,31 & 0,27 & 0,31 & 0,32 & 0,25 & 0,32 & 0,22 \\
\hline & & \multicolumn{10}{|c|}{ Identifikation } \\
\hline \multirow{3}{*}{ Abwanderer } & Mittelwert & 1,65 & 1,71 & 1,72 & 1,76 & 1,80 & 1,76 & 1,93 & 1,99 & 2,20 & 2,23 \\
\hline & $n$ & 595 & 526 & 411 & 346 & 312 & 228 & 214 & 138 & 84 & 42 \\
\hline & Std. & 0,87 & 0,89 & 0,84 & 0,92 & 0,89 & 0,86 & 0,83 & 0,96 & 0,83 & 0,93 \\
\hline \multirow{3}{*}{$\begin{array}{l}\text { Inländer } \\
\text { ohne deut. } \\
\text { Staatsb. }\end{array}$} & Mittelwert & 1,88 & 1,99 & 2,06 & 2,10 & 2,13 & 2,13 & 2,34 & 2,42 & 2,57 & 2,58 \\
\hline & $n$ & 1.887 & 1.851 & 1.805 & 1.803 & 1.756 & 1.582 & 1.624 & 1.478 & 1.858 & 1.677 \\
\hline & Std. & 1,00 & 1,04 & 1,03 & 1,06 & 1,06 & 1,07 & 0,97 & 0,92 & 0,90 & 0,92 \\
\hline \multirow{3}{*}{$\begin{array}{l}\text { Einge- } \\
\text { bürgerte }\end{array}$} & Mittelwert & 2,37 & 2,29 & 2,49 & 2,44 & 2,36 & 2,40 & 2,63 & 2,99 & 3,06 & 3,15 \\
\hline & $n$ & 118 & 122 & 144 & 159 & 164 & 162 & 173 & 135 & 150 & 263 \\
\hline & Std. & 1,17 & 1,15 & 1,25 & 1,15 & 1,18 & 1,14 & 0,98 & 0,84 & 0,83 & 0,81 \\
\hline
\end{tabular}

Quelle: SOEP Wellen 2, 4, 6, 8, 10, 12, 14, 16, 18 und 20 
für die Gruppe der eingebürgerten Befragten ist auf die oben angesprochene Änderung der Sprunganweisungen im Erhebungsinstrument zurückführen. Bedingt durch die Verengung der Betrachtung auf den Arbeitsmarkt, basiert die Analyse der Platzierung auf der kleineren Fallzahl von am Arbeitsmarkt teilnehmenden Personen. Das schon zuvor beobachtete Muster des Anstiegs der Werte bis 1991 mit danach niedrigeren Werten ist auch bei dieser Aufteilung wieder zu entdecken. Ganz entsprechend der theoretischen Erwartungen sind die Abwanderer auf dem Arbeitsmarkt am schlechtesten positioniert. Für die beiden anderen Gruppen fallen die Werte jedoch nicht so theoriekonform aus, das Bild ist von stärkeren Schwankungen in der kleineren Gruppe der irgendwann Eingebürgerten geprägt, dem eine eher konstante Entwicklung in der größeren Gruppe gegenübersteht.

Da die Besuche in den Wohnungen von Deutschen und die Besuche durch Deutsche in der eigenen Wohnung für eingebürgerte Personen ab 1997 nicht mehr erhoben wurden, sinken für diese Gruppe die Fallzahlen für die Betrachtung der Interaktion bis 2003 erheblich ab. Insgesamt lässt sich gruppenintern keine einheitliche Tendenz der Interaktion feststellen. In allen drei Gruppen schwanken die Werte auf hohem Niveau. Zwischen den einzelnen Gruppen zeichnen sich jedoch die prognostizierten Unterschiede ab. Durchgängig liegen die Werte der Eingebürgerten über jenen der ausländischen Staatsbürger mit dauerhaftem Aufenthalt in der Bundesrepublik und die Werte dieser Gruppe über denen der Abwanderer.

Die emotionale Identifikation ist dann wiederum ähnlich wie bei der Kulturation von in der Tendenz steigenden Werten geprägt, wobei die Standardabweichungen etwas homogenere Antworten als bei der Kulturation erkennen lassen. Die Gruppenunterschiede weisen trotz kleinerer Schwankungen innerhalb der Gruppen über den gesamten Betrachtungszeitraum die erwartete Struktur auf; so berichten die Eingebürgerten die höchste emotionale Identifikation mit den Deutschen, wohingegen Abwanderer dies in sehr viel geringerem Umfang tun.

Insgesamt bestätigen die in Tabelle 6.1.2b aufgeführten Ergebnisse, wenn auch nicht in allen Einzelheiten, so doch weitgehend die theoretischen Erwartungen. Hinsichtlich der Unterschiede zwischen den Gruppen fallen Kulturation, Interaktion und Identifikation in der erwarteten Form aus. Für die Platzierung lässt sich dies nur zum Teil feststellen. Während die Abwanderer über die schlechteste durchschnittliche Positionierung auf dem Arbeitsmarkt verfügen, liegen die Werte der beiden an- 
deren Gruppen in einigen Jahren sehr nahe beieinander und in drei der betrachteten zehn Jahre weist die Gruppe ohne Änderungen höhere Werte als die der Eingebürgerten auf. Wie sehr die Platzierung von konjunkturellen Einflüssen geprägt ist, wird an den vom Vereinigungsboom zu Beginn der 1990er Jahre getragenen relativ hohen Positionierungswerten in den einzelnen Gruppen erkennbar.

\subsubsection{Zwischenfazit zu den deskriptiven Ergebnissen}

Mit den vorgestellten Ergebnissen zur Sozialintegration der Immigranten und ihrer Nachkommen sollten einerseits die Entwicklungen in den einzelnen Gruppen herausgearbeitet werden und gleichzeitig auch eine deskriptive Analyse in Bezug auf die Plausibilität der mit den Modellannahmen verbundenen Erwartungen erfolgen.

Anhand der Entwicklung der Mittelwerte konnte gezeigt werden, dass die Sozialintegration in den betrachteten Gruppen von starken Differenzen gekennzeichnet ist. Dabei weist die Gruppe der Personen türkischer Herkunft in allen Subdimensionen die geringste Sozialintegration auf, wohingegen die Gruppe der sonstigen EUBürger für die vier betrachteten Zeitpunkte von 1997 bis 2003 durchgängig die höchste Sozialintegration zeigt. Personen (ex-)jugoslawischer Herkunft und die Befragten aus den EU-Anwerbeländern Griechenland, Italien, Portugal und Spanien sind zwischen den beiden anderen Gruppen angesiedelt. In Hinblick auf die Abwanderungsvorgänge wirken sich die Unterschiede zwischen den Nationalitätsgruppen nicht entsprechend der Erwartung aus, dass gut integrierte Personen weniger und schlecht integrierte vermehrt abwandern. Für SOEP-Teilnehmer türkischer Herkunft und Befragte aus der Gruppe der sonstigen EU-Bürger ergeben sich trotz unterschiedlicher Integration in die Gesellschaft fast identische Abwanderungsraten. Im Unterschied dazu wandern Personen aus den EU-Anwerbeländern sehr viel häufiger aus der Bundesrepublik ab. Für die Einbürgerung ergeben sich ebenfalls $A b-$ weichungen von den formulierten Erwartungen. Die im Verhältnis gut bis am besten integrierten EU-Bürger ließen sich sehr viel seltener als alle anderen Gruppen einbürgern. 
Die Unterschiede in der Einbürgerungsneigung stehen sicherlich mit den aufenthaltsrechtlichen Bestimmungen in der Bundesrepublik Deutschland als einem Mitgliedsstaat der Europäischen Union in Zusammenhang. Im Falle der Abwanderung scheinen weniger push- als pull-Faktoren ausschlaggebend zu sein, so dass die südlichen EU-Herkunftsstaaten für Remigranten möglicherweise mehr Anziehungskraft besitzen als die Türkei für in Deutschland lebende Personen türkischer Herkunft. Darüber hinaus ist es möglich, dass sich insbesondere in Bezug auf gut qualifizierte EU-Bürger, eine Tendenz zur Nutzung von EU-Binnenmigration zur Erweiterung des Arbeitsmarktes in den Daten abzeichnet. Aufschlussreich wäre sicherlich die Entwicklung der Abwanderungsrate türkischer Immigranten und ihrer Nachkommen nach einem EU-Beitritt der Türkei. Wenn die EU-Mitgliedschaft und die damit einhergehende Freizügigkeit für die Bürger der Mitgliedsstaaten einer der wesentlichen Bestimmungsfaktoren für die höhere Abwanderung sein sollte, dann müsste sich dies in den nächsten Jahren an den Abwanderungsraten der Slowenen im Vergleich zu denen der Immigranten aus anderen Teilrepubliken des ehemaligen Jugoslawien zeigen.

Erwartungskonform fällt hingegen der Vergleich von Einwanderergeneration und den nachfolgenden Generationen aus. Die Nachkommen der Immigranten erlangten fast durchgängig ein höheres Sozialintegrationsniveau, dementsprechend ließen sie sich häufiger einbürgern und wanderten sehr viel seltener aus der Bundesrepublik $a b$ als die erste Generation. Die Ergebnisse zu den Nationalitätsgruppen und den Generationen bestätigen zwar nicht in allen Punkten die theoretischen Erwartungen, decken sich aber mit nationalen und internationalen Forschungsergebnissen zur Abwanderung und Naturalisierung von Immigranten (vgl. Dornis 2001, Diehl 2002).

Die Analyse der Sozialintegration von Personen, die sich einbürgern ließen bzw. aus Deutschland abwanderten, im Vergleich zu Befragten, bei denen weder Abwanderung noch Einbürgerung auftraten, zeigt ebenfalls Ergebnisse, die weitgehend im Einklang mit den theoretischen Erwartungen stehen. Abwanderer sind demnach schlechter in die Gesellschaft integriert als die beiden anderen Gruppen und Eingebürgerte sind mit geringfügigen Ausnahmen durchgehend die Gruppe mit der höchsten Sozialintegration. 
Aus den Zusammenhängen von Nationalität, Abwanderung und Einbürgerung ergeben sich Konsequenzen, denen im Rahmen der Ermittlung einer realistischen Integrationsbilanz mehr Beachtung geschenkt werden sollte. Wie bereits Salentin und Wilkening 2003 festgestellt haben, verzerren Differenzen bei den Einbürgerungsraten die Integrationsbilanz einiger Nationalitäten, wenn die Staatsbürgerschaft und nicht die Herkunft als Unterscheidungsmerkmal verwendet werden. Ein ganz ähnlicher Effekt dürfte von den zwischen einzelnen Nationalitäten stark variierenden Abwanderungsraten ausgehen. Denn der Zusammenhang von Abwanderungsentscheidung und Sozialintegration führt dazu, dass die durchschnittliche Sozialintegration der im Aufnahmeland Verbliebenen ansteigt, was bei unterschiedlichen Abwanderungsraten eine vermeintliche Verbesserung der Integrationsbilanz für Gruppen mit starken Abwanderungsbewegungen zur Folge hat. Die relativ schlechten Sozialintegrationswerte der türkischen Inländer werden deshalb zum einen durch die Einbürgerung verzerrt und zum anderen fällt, bedingt durch eine geringere $A b-$ wanderungsrate, der Vergleich mit anderen Nationalitäten - wie z.B. Spanier, Jugoslawen und Griechen - ungünstiger aus. Ein Vergleich der Integrationsbilanz zwischen Herkunftsgruppen ist, wenn er auf Daten aus Trenderhebungen oder amtlichen Statistiken beruht, aber von den Verzerrungen durch die jeweiligen Abwanderungsraten nicht zu bereinigen. Im Hinblick auf die Sozialintegration der türkischstämmigen Bevölkerung in Deutschland ist diese Verzerrung zwar nicht von Bedeutung, aber für die immer wieder aufkeimenden politischen Debatten um die wahrgenommene geringere Integrationsfähigkeit türkischer Einwanderer.

Mit den hier vorgelegten Ergebnissen konnte die Entwicklung einer zunehmenden Sozialintegration der größeren Immigrantengruppen in der Bundesrepublik dargestellt werden. Gleichzeitig wurde eine Reihe von Unterschieden zwischen den Nationalitätengruppen deutlich, die nicht ganz mit den theoretischen Erwartungen im Einklang stehen. Nach dem auf der Sozialintegration beruhenden Erklärungsmodell hätte höhere Sozialintegration zu geringerer Abwanderung und erhöhter Einbürgerung führen sollen. Offensichtlich bestehen aber zwischen der Gruppe der EUBürger und anderen Nationalitäten Differenzen hinsichtlich der Bewertung von Einbürgerung und Abwanderung. Die ausgeprägten Unterschiede lassen es als möglich erscheinen, dass auch im Rahmen der Modellprüfung ähnliche Differenzen zutage treten. Dies würde auch den Ergebnissen Hills entsprechen, der in seinen 
Analysen zur Assimilation zwischen italienischen, jugoslawischen und türkischen Befragten einige Differenzen feststellen konnte (vgl. Hill 1984, 122-190). Für die weiteren Analysen ergibt sich deshalb daraus die Konsequenz, auch bei den folgenden Modellprüfungen das Vorhandensein entsprechender Gruppenunterschiede zu kontrollieren.

\subsection{Strukturgleichungsmodelle zur Erklärung der Abwanderungs- und Einbürgerungsvorgänge}

In diesem Abschnitt wird nun die Umsetzung der theoretisch abgeleiteten kausalen Hypothesen zu den Zusammenhängen der Sozialintegration mit dem Auftreten bzw. Ausbleiben von Abwanderung und Einbürgerung in Form von Strukturgleichungsmodellen dargestellt.

Zum Test der Modelle wurde aufgrund der Komplexität ein mehrstufiges Verfahren gewählt, bei dem zu Beginn mit konfirmatorischen Faktorenanalysen die Messmodelle der einzelnen Konstrukte überprüft wurden. ${ }^{57}$ Darauf aufbauend wurden schrittweise Strukturgleichungsmodelle mit den kausalen Beziehungen zwischen den latenten Variablen getestet (vgl. Jöreskog 1993, Hayduk 1996). ${ }^{58}$ Zur Anwendung kam dabei die von Jöreskog als „model generating situation“ bezeichnete Vorgehensweise, in deren Rahmen zunächst ein theoretisch abgeleitetes Ausgangsmodell getestet wird und wenn erforderlich, entlang der Prüfergebnisse und theoretischen Vorgaben mit den Daten besser zu vereinbarende Modelle spezifiziert (Jöreskog 1993). Damit verliert die Modellprüfung allerdings ihren strikt konfirmatorischen Charakter und entspricht einer im weiteren Sinne explorativ orientierten Strategie.

57 Die Modelltests wurden alle mit LISREL 8.72 durchgeführt. Die Schätzungen beruhen soweit nicht anders vermerkt auf den für ordinale Variablen berechenbaren polychorischen Korrelationen und der Weighted-Least-Square-Diskrepanzfunktion (WLS) (vgl. Reinecke 2005, 111-115).

58 Die Ergebnisse der einzelnen Faktorenanalysen und kausalen Teilmodelle werden aus Rücksicht auf den Umfang nicht alle aufgeführt. Die Ergebnisse dieser Analysen finden sich auf der Dokumentations-CD. 
Um zusätzlich die Stabilität des Modells der Sozialintegration über Zeit und Gruppen hinweg zu testen, wurde für fünf Wellen (1985, 1989, 1995, 1999 und 2003) jeweils getrennt und auch nach den zuvor schon gebildeten Herkunftsgruppen (türkisch, jugoslawisch, EU-Anwerbeländer) ${ }^{59}$ Strukturgleichungsmodelle geschätzt. ${ }^{60} \mathrm{Im}$ Folgenden werden zunächst die Ergebnisse zu Stabilität der Sozialintegration referiert, da diese als Basis der Erklärungsmodelle dienen werden. Im Anschluss an die Ergebnisse zur Sozialintegration der Immigranten und ihrer Nachkommen werden dann die Schätzergebnisse der Erklärungsmodelle und des Prozessmodells zu den Einbürgerungs- und Abwanderungsvorgängen vorgestellt.

\subsubsection{Die kausale Struktur der Sozialintegration}

Bisher wurden die Subdimensionen der Sozialintegration nur isoliert voneinander betrachtet, für die auf der Sozialintegration beruhende Erklärung der Abwanderungs- und Einbürgerungsvorgänge ist aber auch die interne Kausalstruktur der Sozialintegration selbst von ausschlaggebender Bedeutung. Gemäß dem von Esser vorgestellten Modell zur Assimilation bzw. Sozialintegration von Immigranten bestehen zwischen den einzelnen Subdimensionen Beziehungen, deren Struktur und Stabilität an den Daten des Sozio-oekonomischen Panels geprüft wurde.

Als Ausgangsmodell diente hierzu der unter $t_{2}$ gefasste Teil des Prozessmodells der Assimilation von 1980, demzufolge Kulturation alle weiteren Subdimensionen der Sozialintegration verstärkt, die Platzierung einen positiven Effekt auf Interaktion und Identifikation ausübt und die Interaktion eine ebenfalls fördernde Wirkung auf die Identifikation besitzt (vgl. Abbildung 6.2.1a). Die Messung der einzelnen latenten Konstrukte erfolgt über die in Kapitel 5.1 diskutierten und in Tabelle 5.1.2.1a zusammenfassend aufgeführten acht Indikatoren. In dem zu prüfenden Modell stehen somit für jedes latente Konstrukt zwei Indikatoren zur Verfügung. Als Referenzjahr wurde 1999 gewählt, da speziell für den Test des Prozessmodells nur ausgehend von der Welle 16 eine ausreichende Fallzahl zu realisieren war.

59 Für den Test in der Gruppe der sonstigen EU-Bürger waren nicht ausreichend Fälle vorhanden.

60 Zur Stabilität der Messung der Sprachkenntnisse und Identifikation mit der Aufnahmegesellschaft ist hier auch auf die ausführlichen Analysen der SOEP-Daten Jost Reineckes zu verweisen (Reinecke 2005). 


\section{Abbildung 6.2.1a: Struktur- und Messmodell der Sozialintegration ${ }^{61}$}

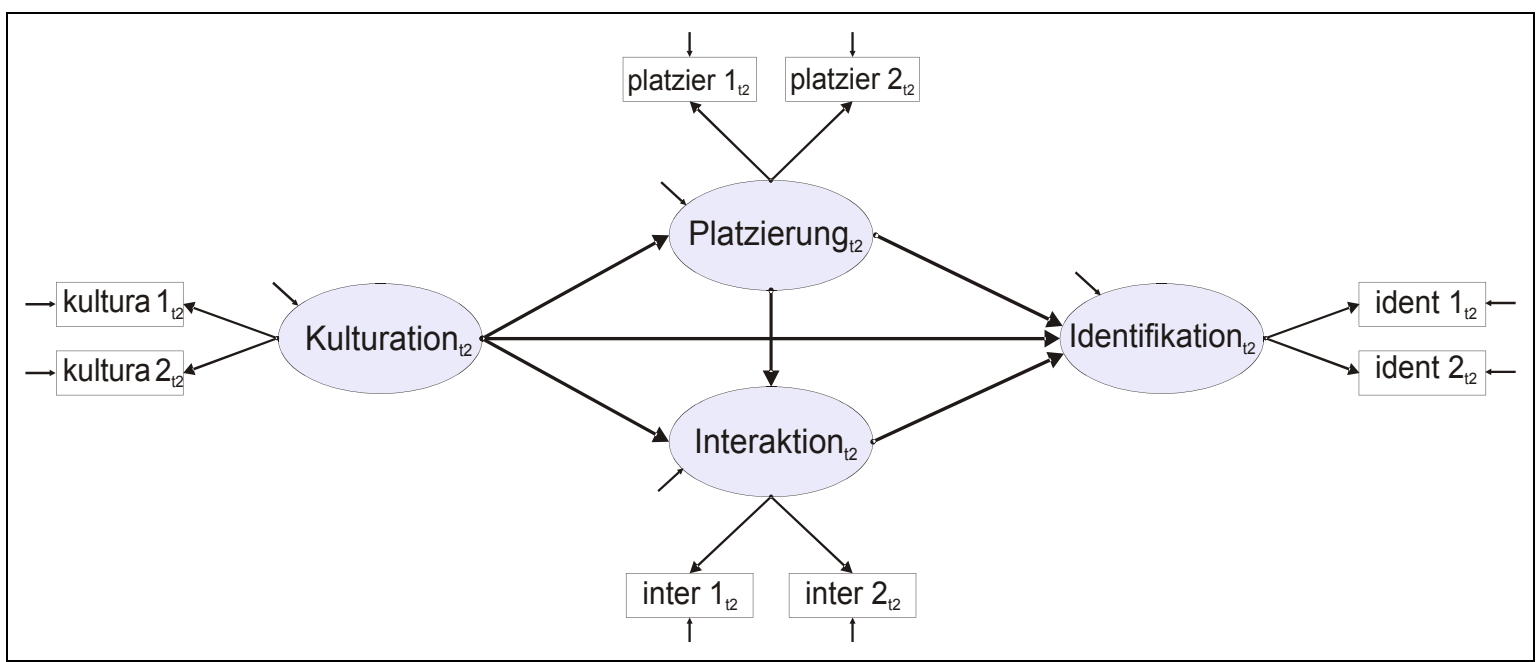

Zur Identifizierung der Variablenbezeichung siehe Tabelle 5.1.2.1a.

Zur Überprüfung der Struktur- und Messhypothesen wurde das in Abbildung 6.2.1a wiedergegebene Modell spezifiziert. Im Rahmen dieser strikt konfirmatorischen Vorgehensweise ergeben sich nur in Ausnahmefällen Modelle, die mit den empirischen Daten im Einklang stehen. ${ }^{62}$ Ausschließlich auf Grundlage des Datensatzes der Welle 2003 konnte - global betrachtet - das theoretisch hergeleitete Modell als ausreichend an die Daten angepasst bezeichnet werden (Chi-Quadrat = 26.58; $\mathrm{df}=16 ; \mathrm{p}$-value $=0.046 ;$ RMSEA $=0.0246 ;$ P-Close $=0.997)$. Allerdings erwies sich die direkte Wirkung der Platzierung auf die Identifikation entgegen der theoretischen Erwartung als nicht signifikant und noch dazu schwach negativ. Weder an den Gesamtdaten der einzelnen Jahrgänge noch getrennt nach Nationalitätsgruppen konnte ein direkter Effekt bestätigt werden. Offensichtlich steht die aktuelle Einbindung in den Arbeitsmarkt nicht unmittelbar in engerer Beziehung mit der emotionalen Hinwendung zur Aufnahmegesellschaft. Alle anderen postulierten Beziehungen zwischen den Subdimensionen der Sozialintegration erwiesen sich in den Modellprüfungen hingegen als weitgehend stabil und von der Richtung her betrachtet positiv.

61 In dieser und den folgenden Abbildungen gelten die üblichen Konventionen für die Darstellung von Strukturgleichungsmodellen: Latente Variablen werden durch Ellipsen und gemessene Variablen durch Rechtecke repräsentiert. Pfeile geben die Beziehungen zwischen den Variablen und die Messfehler wieder.

62 Fit-Indizes des Referenzmodells 1999: Chi-Quadrat $=43.117, \mathrm{df}=16, \mathrm{p}$-value $=0.000, \mathrm{RMSEA}=$ 0.0393, P-Close $=0.886(N=1101)$. 
Abbildung 6.2.1b: Modifiziertes Strukturmodell der Sozialintegration 1999

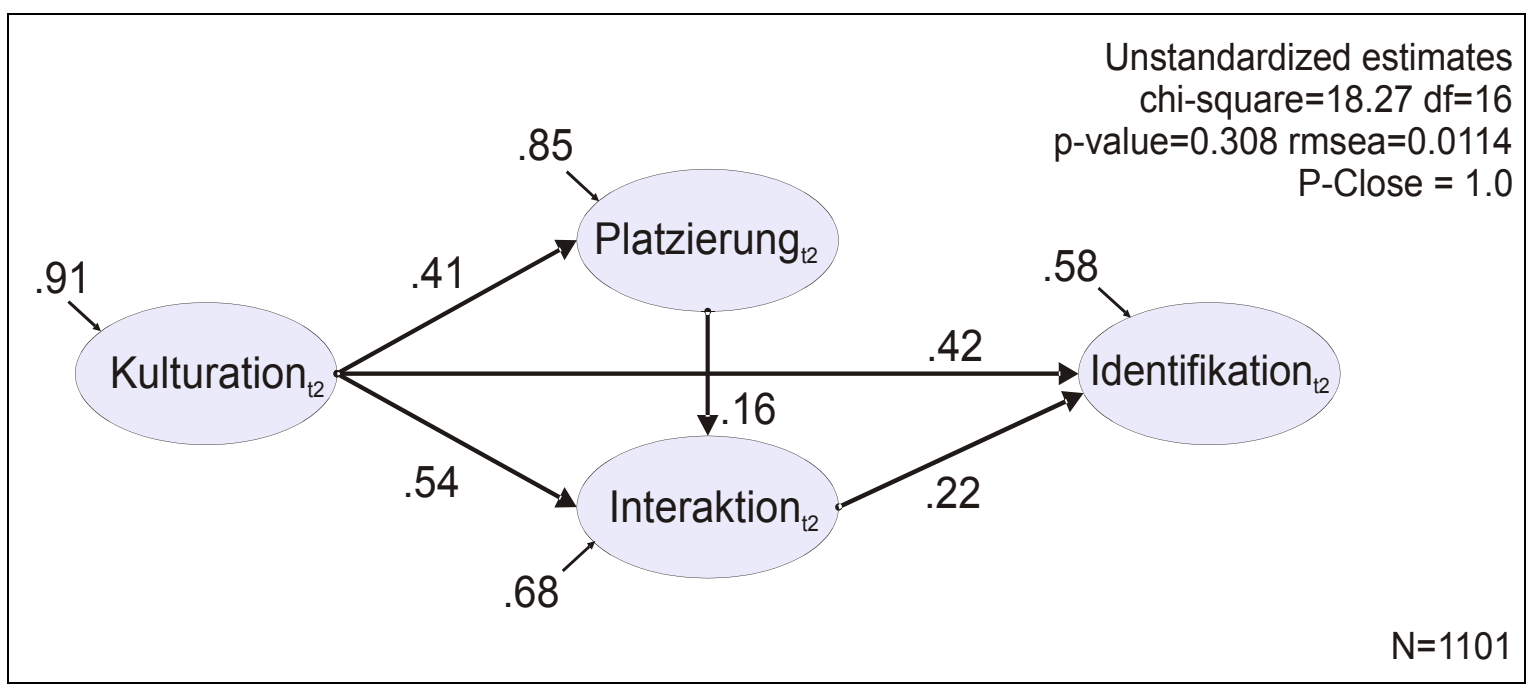

Weighted-Least-Squares Schätzung, listwise deletion

Diese Ergebnisse wurden zum Anlass genommen ein modifiziertes Modell der Sozialintegration zu spezifizieren, das keinen direkten Effekt der Platzierung auf die Identifikation mit der Aufnahmegesellschaft beinhaltet. Auf Basis dieses kausal modifizierten Strukturmodells der Sozialintegration ließ sich für das ausgewählte Referenzjahr 1999 ein Modell schätzen, das mit einem Chi-Quadrat-Wert von 18.27 bei 16 Freiheitsgraden und einem p-Wert von 0.308 eine gute Modellanpassung aufweist (vgl. Abb. 6.2.1b). Um diese Modellanpassung erreichen zu können, ist allerdings eine Nebenladung der Kulturation auf den zweiten Indikator der Platzierung erforderlich. Alle spezifizierten Effekte der latenten Konstrukte untereinander sind signifikant und haben die theoretisch erwartete Richtung. Auffallend sind die im Vergleich starken Effekte der Kulturation auf die weiteren Integrationsbereiche, hingegen ist die Bedeutung der Platzierung entgegen der von Esser geäußerten Erwartung als eher gering einzuschätzen. Die sprachliche Assimilation fördert dem Modell zufolge die Sozialintegration von Immigranten in stärkerem Maße, als dies durch die Integration in den Arbeitsmarkt der Fall ist. Durch die jeweiligen Prädiktoren im Strukturmodell wird die Gesamtvarianz in der latenten Variable Platzierung zu 15\%, der Interaktion zu 31\% und der Identifikation zu 42\% erklärt, was als durchaus befriedigendes Ergebnis gewertet werden kann. Im Vergleich zu dem aus den theoretischen Vorgaben hergeleiteten Strukturgleichungsmodell ist das modifizierte Modell deutlich besser an die empirischen Daten des Jahres 1999 angepasst. 
Um die Stabilität des Modells über die Zeit prüfen zu können, wurden für die Jahre 1985, 1989, 1995 und 2003 zusätzlich entsprechende Modelle geschätzt, dabei konnte die kausale Struktur der Sozialintegration fast durchgängig an den Daten bestätigt werden (vgl. Tab. 6.2.1a).

Tabelle 6.2.1a: Stärke der unstandardisierten Effekte und Güte der jeweiligen Modellanpassung des modifizierten Modells der Sozialintegration für die Jahrgänge 1985, 1989, 1995, 1999 und 2003

\begin{tabular}{|c|c|c|c|c|c|c|}
\hline & Kulturation & Platzierung & Interaktion & Identifikation & $\mathbf{R}^{2}$ & Modellfit und Fallzahl \\
\hline Kulturation 85 & & & & & & \multirow{4}{*}{$\begin{array}{c}\text { Degrees of Freedom }=12 \\
\text { Chi-Square }=22.816 \\
P=0.0293 \\
\text { RMSEA }=0.0219 \\
\text { P-Close }=1.00 \\
N=1886\end{array}$} \\
\hline Platzierung 85 & .055 & & & & .003 & \\
\hline Interaktion 85 & .512 & .095 & & & .283 & \\
\hline Identifikation 85 & .343 & & .205 & & .229 & \\
\hline \multicolumn{6}{|l|}{ Kulturation 89} & \multirow{4}{*}{$\begin{array}{c}\text { Degrees of Freedom }=13 \\
\text { Chi-Square }=18.452 \\
P=0.141 \\
\text { RMSEA }=0.016 \\
\text { P-Close }=1.00 \\
N=1636\end{array}$} \\
\hline Platzierung 89 & .193 & & & & .034 & \\
\hline Interaktion 89 & .443 & .114 & & & .209 & \\
\hline Identifikation 89 & .337 & & .258 & & .250 & \\
\hline \multicolumn{6}{|l|}{ Kulturation 95} & \multirow{4}{*}{$\begin{array}{c}\text { Degrees of Freedom }=16 \\
\text { Chi-Square }=23.346 \\
P=0.105 \\
\text { RMSEA }=0.0187 \\
\text { P-Close }=1.0 \\
N=1311\end{array}$} \\
\hline Platzierung 95 & .349 & & & & .107 & \\
\hline Interaktion 95 & .534 & & & & .249 & \\
\hline Identifikation 95 & .436 & & .176 & & .297 & \\
\hline \multicolumn{6}{|l|}{ Kulturation 99} & \multirow{4}{*}{$\begin{array}{c}\text { Degrees of Freedom }=16 \\
\text { Chi-Square }=18.272 \\
P=0.308 \\
\text { RMSEA }=0.0114 \\
\text { P-Close }=1.00 \\
N=1101\end{array}$} \\
\hline Platzierung 99 & .412 & & & & .154 & \\
\hline Interaktion 99 & .535 & .156 & & & .338 & \\
\hline Identifikation 99 & .418 & & .224 & & .351 & \\
\hline \multicolumn{6}{|l|}{ Kulturation 03} & \multirow{4}{*}{$\begin{array}{c}\text { Degrees of Freedom }=16 \\
\text { Chi-Square }=17.068 \\
P=0.381 \\
\text { RMSEA }=0.0078 \\
\text { P-Close }=1.00 \\
N=1089\end{array}$} \\
\hline Platzierung 03 & .273 & & & & .076 & \\
\hline Interaktion 03 & .498 & .132 & & & .281 & \\
\hline Identifikation 03 & .293 & & .171 & & .176 & \\
\hline
\end{tabular}

Weighted-Least-Squares Schätzung, listwise deletion 
Allerdings war die schwach ausgeprägte Wirkung der Kulturation auf die Platzierung (0.055) in den Daten von 1985 mit einem T-Wert von 1.35 nur noch auf dem 20\%-Niveau signifikant und auf Basis der Daten der Welle 1995 ließ sich der Effekt der Platzierung auf die Interaktion nicht bestätigen. Allerdings mussten dazu in einigen Fällen Fehlerkorrelationen und Nebenladungen zugelassen werden. Speziell die Messung der Sprachkompetenzen und des Erwerbseinkommens machten wie schon für die Daten des Jahres 1999 zusätzliche Modifikationen erforderlich. Alternativ zu diesen Modellen wurden deshalb Modelle geprüft, in denen die Platzierung ausschließlich über die Arbeitsautonomie gemessen wurde. Diese Modelle zeichneten sich durch eine geringfügig bessere Anpassung an die Daten aus und mussten im Hinblick auf die Messmodelle deutlich weniger modifiziert werden. Allerdings stabilisierten sich beide Varianten von 1985 bis 2003 zusehends, was sich insbesondere in einer steigenden Vereinbarkeit mit den Daten ausdrückt. ${ }^{63}$ Allein die Ergebnisse des Jahres 1995 fallen etwas aus diesem Trend heraus, wobei in dieser Welle zum ersten Mal die ausländischen Befragten der Ergänzungsstichprobe $D$ in nennenswertem Umfang ins Gewicht fallen. Es ist deshalb nicht auszuschließen, dass es sich um Stichproben- oder Erhebungseffekte handelt.

Die unstandardisierten Effekte sprechen für eine herausragende Bedeutung der Kulturation für die Sozialintegration insgesamt, auffällig ist dabei, dass die Wirkung auf die Platzierung in den 1980er Jahren eher schwach ausgeprägt war und bis 1999 auf einen Wert von .412 anstieg. Die Zusammenhänge für das Jahr 2003 sind dann trotz der insgesamt besseren Modellanpassung etwas schwächer ausgeprägt als in den Daten des Jahres 1999. Aber auch hierin könnte sich wiederum die Erweiterung des Sozio-oekonomischen Panels um die Stichproben E und G bemerkbar machen (vgl. Tab. 5.1.1a).

Die Entwicklung des Effekts der Kulturation auf die Platzierung unterstreicht die gestiegene Bedeutung der sprachlichen Kompetenzen für die Einbindung der Immigranten in den deutschen Arbeitsmarkt. Noch eindeutiger ist die im Vergleich durchgängig starke Wirkung der Kulturation auf die privaten Kontakte zu deutschen Per-

63 Die Verbesserung der Chi-Quadratstatistik steht jedoch zum Teil auch im Zusammenhang mit der Verringerung des Stichprobenumfangs. Da bei der Berechnung der Chi-Quadratstatistik die Fallzahl berücksichtigt wird ( $\left.X^{2}=(N-1) * F_{W L S}\right)$, führen kleinere Stichproben bei gleichen Modellen zu kleineren Chi-Quadrat-Werten (vgl. Schumacker/Lomax 1996, 125). 
sonen. Im Verhältnis dazu bleibt der Effekt der Platzierung eher hinter den Erwartungen zurück. Für die Identifikation der Immigranten mit der Aufnahmegesellschaft bestimmend ist der Erwerb der deutschen Sprache. Bedingt durch die kausale Struktur transportiert die Interaktion zum Teil auch indirekte Effekte der Kulturation und Platzierung, so dass beispielsweise für das Jahr 1999 abzüglich der indirekten Kräfte ein Effekt von .055 der Interaktion auf die Identifikation daraus resultiert. Insgesamt betrachtet konnte das Modell der Sozialintegration in der modifizierten Form damit an den ausgewählten SOEP-Datensätzen, wenn auch mit einigen Einschränkungen, so doch weitgehend bestätigt werden.

Durch die Unterschiede der Herkunftsgruppen bezogen auf die Ergebnisse der deskriptiven Analysen erscheint es notwendig, das Modell der Sozialintegration auf seine Stabilität in den gebildeten Nationalitätsgruppen zu testen. Dazu wurde das bereits für den zeitlichen Vergleich verwendete Modell getrennt für Personen türkischer und (ex-)jugoslawischer Herkunft sowie für die Befragten aus EU-Anwerbeländern gemeinsam an den Daten der 1999 Welle getestet.

Die Ergebnisse dieser Tests sind für den Vergleich neben denen der Gesamtstichprobe (1999) in Tabelle 6.2.1b abgetragen. Auf den ersten Blick ist zu erkennen, dass die kausale Struktur auch für die einzelnen Nationalitätsgruppen mit den empirischen Daten zu vereinbaren ist. Die Modellanpassung fällt dabei für die Befragten türkischer Herkunft am besten aus (Chi-Quadrat $=16.842$; $\mathrm{df}=17$; $\mathrm{p}$-value $=0.4651 ;$ RMSEA $=0.00 ;$ P-Close $=0.961)$ und für die Teilgruppe (ex-)jugoslawischer Herkunft immer noch akzeptabel aber deutlich niedriger aus (Chi-Quadrat = 25.799; $\mathrm{df}=17 ; \mathrm{p}$-value $=0.078 ; \mathrm{RMSEA}=0.047 ; \mathrm{P}$-Close $=0.514)$.

Ganz ähnlich wie bei den Analysen der einzelnen Jahrgangsdaten ist wiederum ein dominierender Einfluss der Kulturation für alle Teilgruppen festzustellen, während die Effekte der Platzierung und Interaktion im Modell eher schwächer sind. Trotz aller Gemeinsamkeiten lassen sich zwischen den Gruppen auch einige Unterschiede erkennen. Die Wirkung der Platzierung auf die Interaktion ist in dem Modell für die Personen (ex-)jugoslawischer Herkunft etwa doppelt so groß wie bei den beiden anderen Gruppen, was darauf hindeutet, dass die Einbindung in den Arbeits- 
Tabelle 6.2.1b: Stärke der unstandardisierten Effekte und Güte der jeweiligen Modellanpassung des modifizierten Modells der Sozialintegration getrennt nach Herkunftsgruppen für das Jahr 1999

\begin{tabular}{|c|c|c|c|c|c|c|c|}
\hline Gruppe & Konstrukt & Kulturation & Platzierung & Interaktion & Identifikation & $\mathbf{R}^{2}$ & Modellfit und Fallzahl \\
\hline \multirow{4}{*}{$\begin{array}{c}\text { alle } \\
\text { Befragten }\end{array}$} & Kulturation 99 & & & & & & \multirow{4}{*}{$\begin{array}{c}\text { Degrees of Freedom }=16 \\
\text { Chi-Square }=18.272 \\
P=0.308 \\
\text { RMSEA }=0.0114 \\
\text { P-Close }=1.00 \\
N=1101\end{array}$} \\
\hline & Platzierung 99 & .412 & & & & .154 & \\
\hline & Interaktion 99 & .535 & .156 & & & .338 & \\
\hline & Identifikation 99 & .418 & & .224 & & .351 & \\
\hline \multirow{4}{*}{ türkisch } & Kulturation 99 & & & & & & \multirow{4}{*}{$\begin{array}{c}\text { Degrees of Freedom }=17 \\
\text { Chi-Square }=16.842 \\
P=0.465 \\
\text { RMSEA }=0.00 \\
\text { P-Close }=0.961 \\
N=351\end{array}$} \\
\hline & Platzierung 99 & .325 & & & & .096 & \\
\hline & Interaktion 99 & .426 & .183 & & & .293 & \\
\hline & Identifikation 99 & .369 & & .229 & & .303 & \\
\hline \multirow{4}{*}{$\begin{array}{l}\text { jugo- } \\
\text { slawisch }\end{array}$} & Kulturation 99 & & & & & & \multirow{4}{*}{$\begin{array}{c}\text { Degrees of Freedom }=17 \\
\text { Chi-Square }=25.799 \\
P=0.0782 \\
\text { RMSEA }=0.047 \\
\text { P-Close }=0.514 \\
N=235\end{array}$} \\
\hline & Platzierung 99 & .339 & & & & .103 & \\
\hline & Interaktion 99 & .407 & .348 & & & .346 & \\
\hline & Identifikation 99 & .446 & & .219 & & .344 & \\
\hline \multirow{4}{*}{$\begin{array}{l}\text { EU-Anwerbe- } \\
\text { länder }\end{array}$} & Kulturation 99 & & & & & & \multirow{4}{*}{$\begin{array}{c}\text { Degrees of Freedom }=17 \\
\text { Chi-Square }=23.166 \\
P=0.144 \\
\text { RMSEA }=0.0292 \\
\text { P-Close }=0.845 \\
N=425\end{array}$} \\
\hline & Platzierung 99 & .428 & & & & .163 & \\
\hline & Interaktion 99 & .633 & .150 & & & .471 & \\
\hline & Identifikation 99 & .518 & & .160 & & .470 & \\
\hline
\end{tabular}

Weighted-Least-Squares Schätzung, listwise deletion

markt in dieser Teilgruppe einen stärkeren Einfluss auf die Interaktion und damit auch indirekt auf die Identifikation mit der Aufnahmegesellschaft besitzt. Für die Befragten aus EU-Anwerbeländern bleibt zu vermerken, dass in ihrem Fall der Kulturation eine im Vergleich zu den anderen Gruppen noch stärkere Rolle im Modell zukommt und Platzierung sowie Interaktion deutlich weniger zur Erklärung der im Modell vorhandenen Varianz beitragen.

Über die Zeit und auch getrennt nach Herkunftsgruppen ergaben die Modellprüfungen eine Bestätigung des modifizierten kausalen Modells der Sozialintegration. Dabei scheint dem Erwerb sprachlicher Kompetenzen eher als der Einbindung in den Arbeitsmarkt eine Schlüsselfunktion für die Sozialintegration insgesamt zuzukommen. 


\subsubsection{Sozialintegration als Erklärung für Einbürgerungs- und Abwanderungsvorgänge}

Nachdem nun ein Kausalmodell der Sozialintegration zur Verfügung steht, das sowohl über die Zeit als auch in den Nationalitätsgruppen hinreichend stabil zu sein scheint, soll nun das Modell zur Erklärung der Abwanderungs- und Einbürgerungsvorgänge genutzt werden. Mit dieser Vorgehensweise ist die Frage verbunden, welchen Einfluss das Niveau des Integriert-Seins auf das Auftreten bzw. Ausbleiben von Abwanderung und Einbürgerung besitzt. Den theoretischen Erwartungen nach sollte die Abwanderung umso seltener vorkommen und die Einbürgerung umso häufiger auftreten, je höher das Niveau der Sozialintegration und damit die Bindung an die Aufnahmegesellschaft ausgeprägt ist. Im Sinne des Ausgangsmodells wird hierbei davon ausgegangen, dass die einzelnen Subdimensionen gleichermaßen einen direkten Einfluss ausüben können, da vor dem explizierten theoretischen Hintergrund keine Anhaltspunkte für eine herausgehobenere Stellung einer oder mehrere Teildimensionen vorliegen.

Aus diesen Vorannahmen und den Ergebnissen des Modelltests zur kausalen Struktur der Sozialintegration ergibt sich das in Abbildung 6.2.2a wiedergegebene Strukturmodell, in dem die Einbürgerungs-Abwanderungs-Dimension durch alle Subdimensionen der Sozialintegration erklärt wird.

Im Anschluss an die Prüfungen zur Kausalstruktur der Sozialintegration wurden zunächst Modelle inklusive aller bisher verwendeten Indikatoren spezifiziert. Es ergaben sich dabei aber zum Teil Ausgangsdaten, die nicht positiv definit waren, was eine Modellschätzung in diesen Fällen nicht zuließ. ${ }^{64}$ Deshalb wurde in den weiteren Modellprüfungen ein reduziertes Messmodell eingesetzt, das für die Messung der Platzierung und der Interaktion jeweils nur einen Indikator beinhaltet. Die in Abbildung 6.2.2a grau hinterlegten Indikatoren platzier2 und inter2 konnten nicht weiter verwendet werden, da die Datenprobleme auf diese Variablen zurückgeführt werden konnten. Für die Messung der Platzierung und Interaktion bedeutet dies,

64 Die zur Kontrolle durchgeführten Hauptkomponentenanalysen unter Einbeziehung der Variablen plazier2 und inter2 ergab Hauptkomponenten mit Eigenwerten gleich und kleiner 0.0. Entsprechende Schätzprobleme ergeben sich manchmal durch ausfallbedingte Redundanzen bei ordinalen Daten. 


\section{Abbildung 6.2.2a: Struktur- und Messmodell zur Erklärung der Abwanderungs- und Einbürgerungsvorgänge über die Sozialintegration}

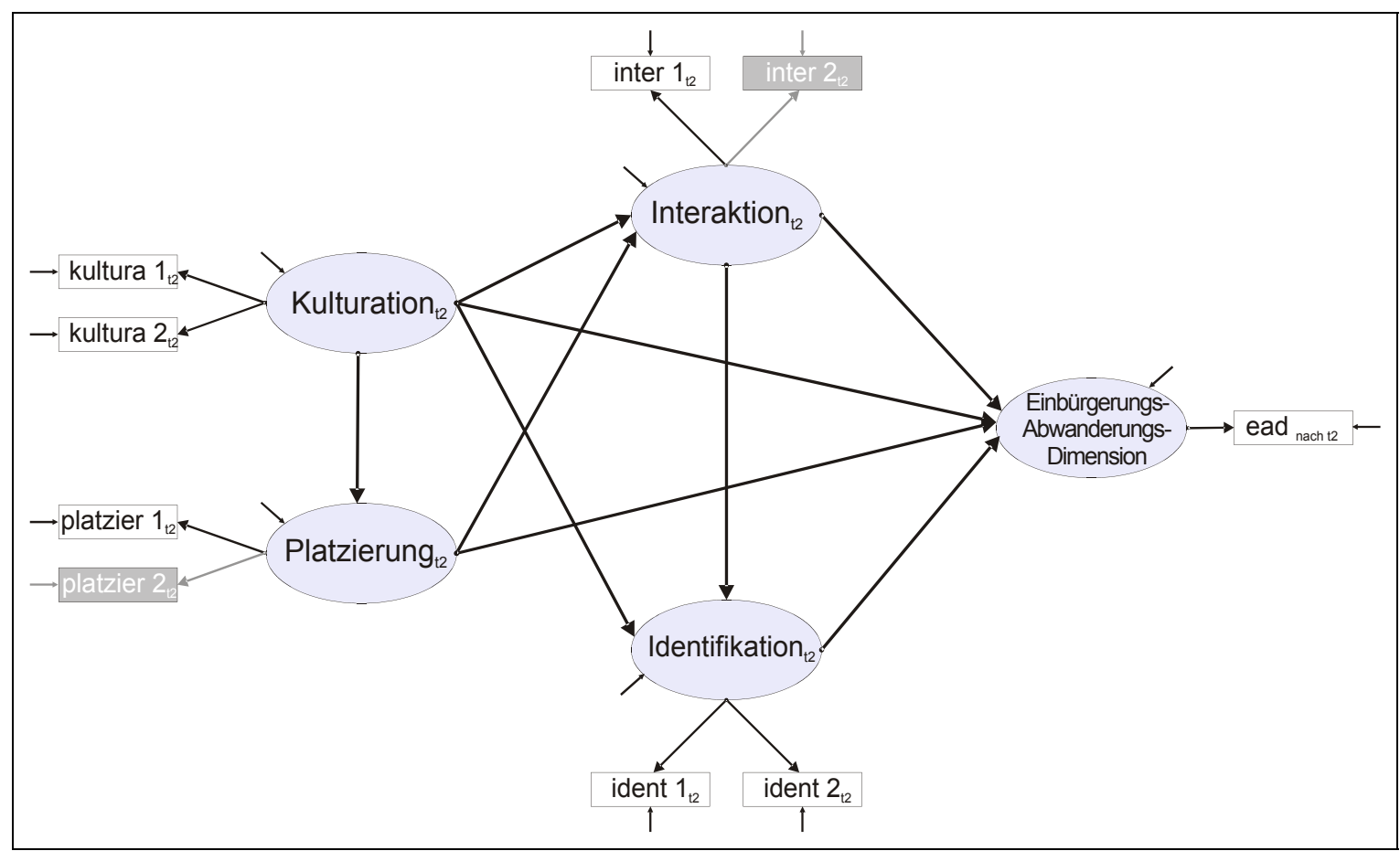

Zur Identifizierung der Variablenbezeichung siehe Tabelle 5.1.2.1a.

dass die Platzierung mit der Messung der Arbeitsautonomie und die Interaktion mit dem Besuch bei Deutschen gleichgesetzt wurden. ${ }^{65}$ Bedingt durch die schrittweise Veränderung der gesetzlichen Regelungen zur Einbürgerung stehen für entsprechende Modelle in den SOEP-Daten erst mit den Wellen Ende der 1990er Jahre ausreichend Einbürgerungsfälle zur Verfügung. Aus diesem Grund wurde das Modell zunächst mit den Daten zur Sozialintegration der Welle 1999 und dem von 2000 bis 2004 Auftreten bzw. Ausbleiben von Einbürgerungs- und Abwanderungsvorgängen getestet.

Die Schätzung des auf den theoretischen Annahmen beruhenden Strukturgleichungsmodells war interferenzstatistisch nicht mit den Daten zu vereinbaren (ChiQuadrat $=19.596 ; \mathrm{df}=8 ; \mathrm{p}$-value $=0.012 ;$ RMSEA $=0.0346 ;$ P-Close $=0.893 ; \mathrm{N}=$ 1209). Aber durch die Spezifikation eines alternativen Modells ohne die direkten Effekte von Platzierung und Interaktion auf die Einbürgerungs-Abwanderungs-Dimen-

65 Die Gleichsetzung der latenten Variable mit der Messung hat zur Folge, dass für diese Konstrukte Messfehler und Messfehlerkorrelationen nicht ermittelte werden können. 
Abbildung 6.2.2b: Strukturmodell zur Erklärung der Abwanderungs- und Einbürgerungsvorgänge zwischen 2000 und 2004 über das Niveau der Sozialintegration 1999

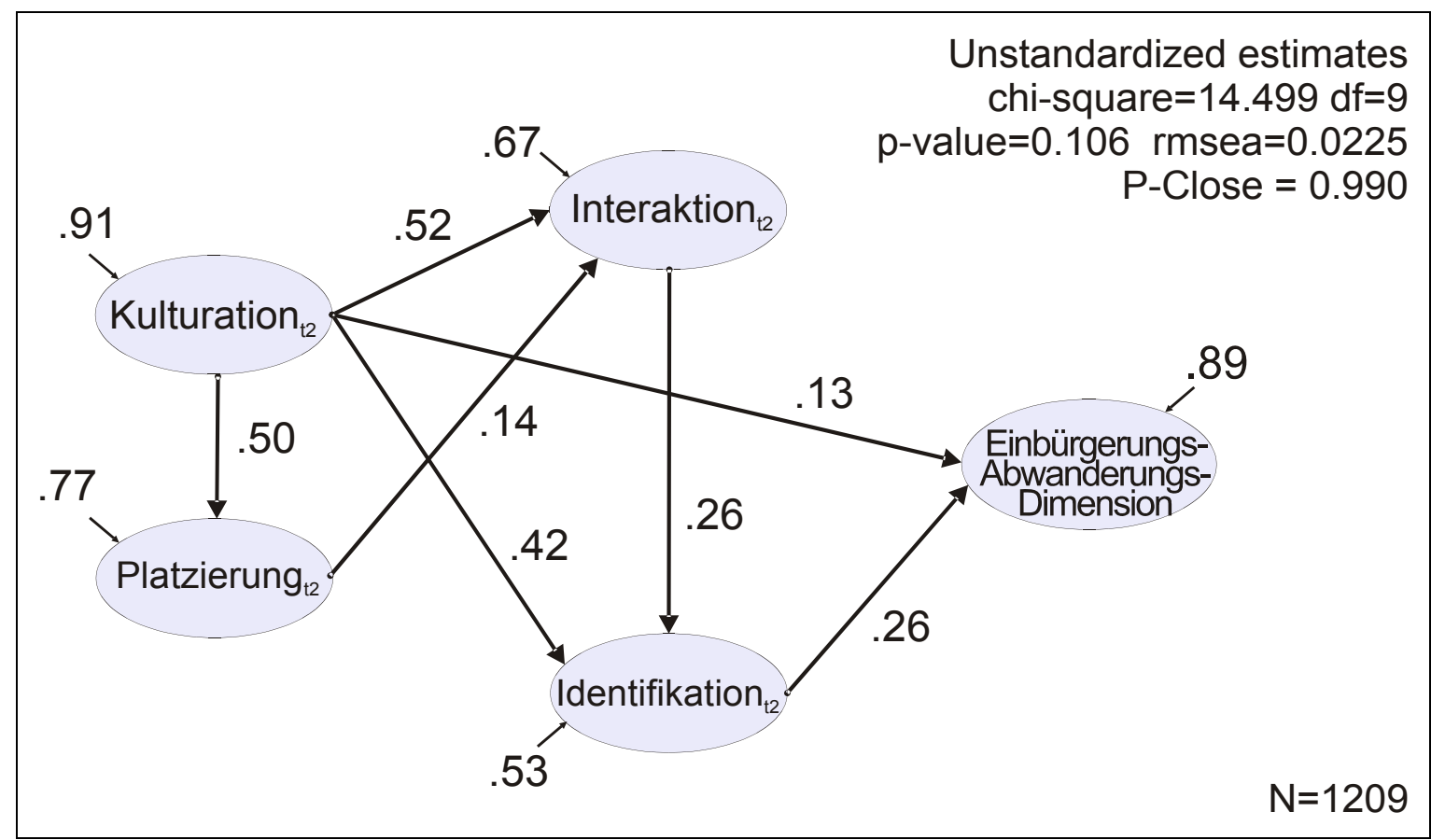

Weighted-Least-Squares Schätzung, listwise deletion

sion (vgl. Abb. 6.2.2b), konnte die Modellanpassung deutlich verbessert werden (Chi-Quadrat $=14.499 ; \mathrm{df}=8 ; \mathrm{p}$-value $=0.106 ;$ RMSEA $=0.0225 ; \mathrm{P}$-Close $=0.960$; $N=1209$ ).

Das Modell gibt in dieser Form die Prüfergebnisse zur Kausalstruktur der Sozialintegration wieder und beinhaltet direkte Effekte von Kulturation und Identifikation auf die neu eingeführte Variable zur Einbürgerungs-Abwanderungs-Dimension. In diesem Modell werden somit die Einbürgerungs- und Abwanderungsvorgänge direkt durch das Ausmaß der subjektiven Deutschkompetenz und die Identifikation mit den Deutschen erklärt. Indirekt üben die Positionierung auf dem Arbeitsmarkt und der Kontakt zu Deutschen über die Identifikation jeweils einen geringen Einfluss aus. $^{66}$

66 Alternative Modelle mit der, im SOEP gemessenen Bleibeabsicht ergaben keinen zusätzlichen Erkenntnisgewinn, da Identifikation und Bleibeabsicht schlecht zu trennen waren, was möglicherweise durch die direkt aufeinanderfolgende Abfrage im Erhebungsinstrument des Sozio-oekonomischen Panels begründet ist. 
Ein analoges Modell zur Erklärung der Vorgänge mittels der Sozialintegrationsdaten aus dem Jahre 1995 ergab für die Gesamtdaten eine gute Modellanpassung (Chi-Quadrat $=11.78 ; \mathrm{df}=12 ; \mathrm{p}$-value $=0.464 ;$ RMSEA $=0.00 ;$ P-Close $=1.0 ; \mathrm{N}=$ 1238) für den Erklärungszeitraum von 1996 bis 2000. Im Unterschied zu dem 1999er Modell konnte der direkte Einfluss der Kulturation auf die Einbürgerungs-Abwanderungs-Dimension nicht bestätigt werden. Mit der Ausdehnung des Erklärungszeitraums auf die Phase von 1996 bis 2004 stellte sich dieser direkte Effekt wieder ein, wobei die Modellanpassung in diesem Fall allerdings schlechter ausfällt (Chi-Quadrat $=20.16 ; \mathrm{df}=11 ; \mathrm{p}$-value $=0.043$; $\mathrm{RMSEA}=0.026$; $\mathrm{P}$-Close $=0.990$; $N=1238) .{ }^{67}$ Dieses Ergebnis kann insgesamt dahingehend interpretiert werden, dass sich darin wiederum die zunehmende Bedeutung einer gelungenen Kulturation für den Aufenthalt in der Bundesrepublik ausdrückt.

Das Modell kann gemäß der Chi-Quadratstatistik in allen Fällen als zumindest ausreichend an die Daten angepasst angesehen werden, deshalb wurden auf dieser Grundlage für die unterschiedlichen Nationalitätengruppen separate Modelle geschätzt. Hintergrund dieser Vorgehensweise waren die zum Teil erheblichen Unterschiede im Hinblick auf Einbürgerung und Abwanderung in den deskriptiven Analysen, gestützt durch die nationalen und internationalen Befunde, wonach Differenzen in Abhängigkeit von der Nationalität im Zusammenhang mit Einbürgerung und Abwanderung eher die Regel zu sein scheinen (zur Einbürgerung: Bernard 1936; Yang 1994; Dornis 2001 und zur Abwanderung: Jasso/Rosenzweig 1990; Borjas/Bratsber 1994; Jankowitsch /Klein/Weick, 2000; Constant/Massey 2002; Nekby 2004; Möser 2005). Auch die Unterschiede in der Stärke einzelner Effekte in den Analysen zur Sozialintegration konnten als Hinweis auf herkunftsspezifische Variationen gedeutet werden.

Um die Möglichkeit des Vorhandenseins herkunftsspezifischer Modellunterschiede prüfen zu können, wurden erneut auf Basis des Datensatzes 1999 getrennt nach den bereits zuvor verwendeten drei Herkunftsgruppen Modelle geschätzt. Anhand der in Tabelle 6.2.2a wiedergegebenen Ergebnisse, kann die Vermutung, dass Unterschiede zwischen den Nationalitätengruppen vorliegen, als bestätigt an-

67 Auch hier wurde das Ergebnis der Prüfung zur Kausalstruktur der Sozialintegration an den SOEP-Daten des Jahres 1995 bestätigt, bei der kein direkter Einfluss der Platzierung auf die Interaktion festgestellt werden konnte. 
Tabelle 6.2.2a: Stärke der unstandardisierten Effekte und Güte der jeweiligen Modellanpassung des Erklärungsmodells getrennt nach Herkunftsgruppen für das Jahr 1999

\begin{tabular}{|c|c|c|c|c|c|c|c|}
\hline Gruppe & Konstrukt & Kulturation & Platzierung & Interaktion & Identifikation & $\mathbf{R}^{2}$ & Modellfit und Fallzahl \\
\hline \multirow{5}{*}{ alle } & Kulturation 99 & & & & & & \multirow{5}{*}{$\begin{array}{c}\text { Degrees of Freedom }=9 \\
\text { Chi-Square }=14.499 \\
P=0.106 \\
\text { RMSEA }=0.0225 \\
\text { P-Close }=0.990 \\
N=1209\end{array}$} \\
\hline & Platzierung 99 & .499 & & & & .226 & \\
\hline & Interaktion 99 & .522 & .141 & & & .332 & \\
\hline & Identifikation 99 & .419 & & .263 & & .397 & \\
\hline & Einb.-Abw.-Dim. & .127 & & & .261 & .109 & \\
\hline \multirow{5}{*}{ türkisch } & Kulturation 99 & & & & & & \multirow{5}{*}{$\begin{array}{c}\text { Degrees of Freedom }=10 \\
\text { Chi-Square }=5.546 \\
P=0.859 \\
\text { RMSEA }=0.00 \\
\text { P-Close }=0.989 \\
N=332\end{array}$} \\
\hline & Platzierung 99 & .318 & & & & .095 & \\
\hline & Interaktion 99 & .442 & .114 & & & .226 & \\
\hline & Identifikation 99 & .343 & & .279 & & .351 & \\
\hline & Einb.-Abw.-Dim. & .185 & & & .163 & .080 & \\
\hline \multirow{5}{*}{$\begin{array}{l}\text { jugo- } \\
\text { slawisch }\end{array}$} & Kulturation 99 & & & & & & \multirow{5}{*}{$\begin{array}{c}\text { Degrees of Freedom }=10 \\
\text { Chi-Square }=15.021 \\
P=0.131 \\
\text { RMSEA }=0.0471 \\
\text { P-Close }=0.487 \\
N=227\end{array}$} \\
\hline & Platzierung 99 & .342 & & & & .106 & \\
\hline & Interaktion 99 & .370 & .391 & & & .362 & \\
\hline & Identifikation 99 & .439 & & .197 & & .347 & \\
\hline & Einb.-Abw.-Dim. & .273 & & & .290 & .216 & \\
\hline \multirow{5}{*}{$\begin{array}{l}\text { EU-Anwerbe- } \\
\text { länder }\end{array}$} & Kulturation 99 & & & & & & \multirow{5}{*}{$\begin{array}{c}\text { Degrees of Freedom }=10 \\
\text { Chi-Square }=15.498 \\
P=0.115 \\
\text { RMSEA }=0.0369 \\
\text { P-Close }=0.697 \\
N=405\end{array}$} \\
\hline & Platzierung 99 & .456 & & & & .194 & \\
\hline & Interaktion 99 & .629 & .192 & & & .731 & \\
\hline & Identifikation 99 & .345 & & .418 & & .499 & \\
\hline & Einb.-Abw.-Dim. & & & & .511 & .223 & \\
\hline
\end{tabular}

Weighted-Least-Squares Schätzung, listwise deletion

gesehen werden. Es ließen sich für die Gruppen getrennt jeweils Modelle mit besserer Modellanpassung finden, die geringfügige aber doch wesentliche Abweichungen vom Gesamtmodell aufweisen. So waren für die Modellanpassung in diesen Fällen keine zusätzlichen Modifikationen erforderlich, aber vor allem in der Bedeutung der einzelnen Unterkonstrukte der Sozialintegration im Rahmen des jeweiligen Modells ergaben sich deutliche Differenzen, die das Gesamtmodell als Ausdruck der Mischung der Gruppenunterschiede erscheinen lässt.

Wie auch schon zuvor bestehen Gemeinsamkeiten hinsichtlich der durchgängig großen Bedeutung der Kulturation für die weiteren Teildimensionen der Sozialintegration, wobei dieser Effekt wiederum bei den betrachteten EU-Bürgern am stärk- 
sten ausgeprägt ist. Die Platzierung wirkt ebenfalls weiterhin nur auf die Interaktion, allerdings mit größeren Unterschieden in den Gruppen. So ist der Effekt der Platzierung auf die Interaktion bei Personen (ex-)jugoslawischer Herkunft mit .391 sogar etwas größer als die Wirkung der Kulturation (.370). Bei allen anderen Teilgruppen liegt er hingegen im Bereich von einem Drittel bis einem Viertel des Einflusses der Kulturation. Auffallend ist hierbei die geringe Bedeutung der Positionierung auf dem Arbeitsmarkt für die Interaktion türkischer Befragter mit deutschen Personen. Der Einfluss des sozialen Kontakts zu deutschen Personen auf die Identifikation ist im Vergleich zwischen den Gruppen eher als stabil zu bezeichnen, wenn auch hier der Effekt im Modell der EU-Gruppe am stärksten ist (.418).

Im Hinblick auf die Erklärung der Einwanderungs-Abwanderungs-Dimension liegen wieder größere Unterschiede zwischen den Gruppen vor. Bei Befragten türkischer und jugoslawischer Herkunft sind es Kulturation und Identifikation, die zur Erklärung beitragen, während für die ausgewählte Gruppe der EU-Bürger nur die Identifikation einen signifikanten Einfluss aufweist. Für die Teilgruppe (ex-)jugoslawischer Herkunft sind die Effekte der Kulturation (.273) und Identifikation (.290) annähernd gleich groß, bei den Personen in der türkischen Gruppe ist die Kulturation (.185) bedeutsamer als die Identifikation (.163). Die direkte Wirkung der Identifikation auf die Vorgänge ist bei den EU-Bürgern mit .511 ungleich höher, aber hierin schlagen sich auch indirekte Effekte der Kulturation in einer Gesamthöhe von .319 nieder, was das Fehlen der direkten Beziehung etwas relativiert.

An dieser Stelle bleibt anzumerken, dass der erklärte Varianzanteil der Einwanderungs-Abwanderungs-Dimension im Modell der Befragten türkischer Herkunft am geringsten ist $\left(R^{2}=.08\right)$ und bei den beiden anderen Gruppen mit rund 22\% erheblich höher liegt. Absolut betrachtet ist der durch die Sozialintegration erklärte Varianzanteil bei den Personen türkischer Herkunft sehr gering und bei den anderen Teilgruppen als akzeptabel zu bezeichnen. Die vorgestellten Ergebnisse der Analysen zur Erklärung des Auftretens bzw. Ausbleibens von Abwanderung und Einbürgerung belegen zum einen die große Bedeutung des Niveaus der aktuellen Kulturation über alle Gruppen hinweg, aber auch das Vorhandensein herkunftsspezifischer Unterschiede in der Bedeutung einzelner Faktoren. 
Interessant ist darüber hinaus, dass für die Personen aus den südlichen EU-Mitgliedsstaaten und (ex-)jugoslawischer Herkunft mit der Sozialintegration eine erheblich bessere Vorhersage möglich ist, als für Personen türkischer Herkunft. Werden die niedrigen Abwanderungs- und hohen Einbürgerungsraten der Personen türkischer Herkunft berücksichtigt, so kann dieser Befund möglicherweise auf eine Einbindung in die Aufnahmegesellschaft zurückgeführt werden, die einen geringeren direkten Bezug zur deutschen Mehrheitsgesellschaft aufweist. Dies wäre dann ein Indiz für die Bindungskräfte einer gut organisierten und in einigen Teilen Charakteristiken einer ethnischen Segmentation zeigenden Community, wie sie von den türkischen Immigranten in der Bundesrepublik aufgebaut worden ist.

\subsubsection{Der Sozialintegrationsprozess als Erklärung für Einbür- gerungs- und Abwanderungsvorgänge}

Die Erklärung der Einbürgerungs- und Abwanderungsvorgänge über das erreichte Niveau der Sozialintegration zu einem bestimmten Zeitpunkt lässt die Entwicklung der Sozialintegration außer Betracht. In „Aspekte der Wanderungssoziologie“ hat Esser ein Modell des Prozesses der Assimilation bzw. Sozialintegration vorgelegt, in dem die Komponente der Entwicklung berücksichtigt ist (Esser 1980). Dieses Prozessmodell der Sozialintegration unterscheidet sich von den bisher verwendeten Erklärungsmodellen durch Informationen über die Interaktion und Platzierung in der Ankunftsphase (t1), die dem Kausalmodell der Sozialintegration (t2) vorangestellt werden. Dahinter steht die Vermutung, dass die von den Immigranten vorgefundenen Startbedingungen in Form der ersten Kontakterfahrungen und der zu Beginn erreichten Positionierung für den weiteren Verlauf der Sozialintegration prägend sind.

Die Umsetzung des Kerns des assimilativ ausgerichteten Prozessmodells der Sozialintegration zur Erklärung der Einbürgerungs- und Abwanderungsvorgänge von Immigranten mit den Daten des Sozio-oekonomischen Panels in Form eines Strukturgleichungsmodells ist in Abbildung 6.2.3a skizziert. 


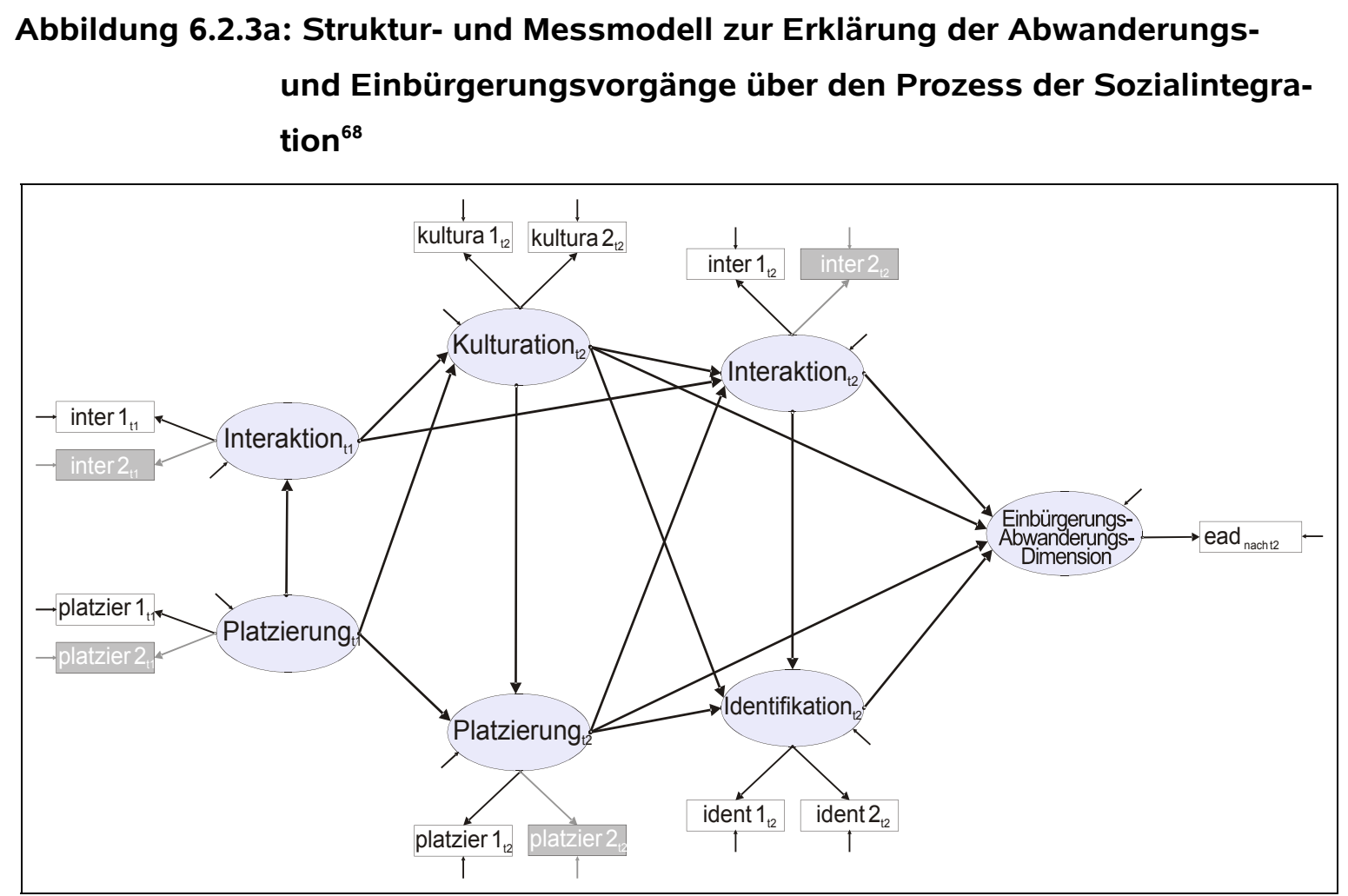

Zur Identifizierung der Variablenbezeichung siehe Tabelle 5.1.2.1a.

Gemäß den theoretischen Erwartungen sollten die neu eingeführten Variablen zur Platzierung und Interaktion der Anfangsphase nicht auf alle Teile der Sozialintegration wirken. Die anfängliche Platzierung sollte in positiver Form mit der späteren Platzierung verbunden sein und ein entsprechender Effekt wird auch für die ersten Interaktionserfahrungen bezüglich der späteren erwartet. Darüber hinaus wird postuliert, dass je intensiver die Kontakte und je besser die Platzierung ausfallen, um so stärker sollte die Kulturation sein. Zusätzlich wird noch ein fördernder Einfluss der Platzierung auf die Interaktion in der Anfangsphase erwartet.

Obwohl die SOEP-Daten für die Analyse der zeitlichen Veränderungen angelegt sind, ergaben sich bei der Umsetzung einige Probleme. Von der Konzeption des Ansatzes ist die Zielgruppe auf Personen beschränkt, über die Informationen zur Platzierung und Interaktion in der Ankunftsphase vorliegen. Es konnten somit nur Respondenten in die Analyse aufgenommen werden, die möglichst nicht allzu lange

68 Wie bei den Erklärungsmodellen in Abschnitt 6.2.2 konnten nicht alle Variablen in die Analyse aufgenommen werden, da die Eingabematrix als nicht positiv definit erkannt wurde. Es wurden wieder die in der Abbildung grau hinterlegten Indikatoren platzier2 und inter2 aus der Analyse herausgenommen. 
Zeit vor ihrer ersten Befragung eingereist sein sollten und für die alle weiteren Informationen in den späteren Wellen vorlagen. Bedingt durch die erst ab dem Ende der 1990er Jahre nennenswerten Einbürgerungszahlen war die Auswahl an Jahrgängen für diesen Test sehr beschränkt. Für die Welle des Jahres 1999 konnte bezogen auf die ausgewählten Variablen eine Fallzahl von N=143 ermittelt werden. In späteren Wellen reduzierten sich die Anzahl der Personen über die verbleibenden Einbürgerungs-Abwanderungs-Vorgänge und in Wellen vor 1996 waren in Verbindung mit Item- und Unit-Nonresponse nicht genügend kürzlich eingewanderte Personen mit entsprechenden Informationen vorhanden. ${ }^{69} \mathrm{Um}$ bei gegebenem Umfang an latenten Konstrukten und zu schätzenden Parametern eine stabilere Schätzung ${ }^{70}$ gewährleisten zu können, wurden in Anbetracht der geringen Fallzahl die als ordinal eingestuften Indikatorvariablen der Sozialintegration dichotomisiert.

Das Ausgangsmodell kann auf Basis der robusten ML-Schätzung zwar als hinreichend interferenzstatistisch abgesichert angesehen werden (Satorra-Bentler Scaled Chi-Quadrat $=14.2398 ; \mathrm{df}=18 ; \mathrm{p}$-value $=0.7133)^{71}$, aber insgesamt beinhaltet das Modell einige nicht signifikante Effekte. Werden diese Effekte aus dem Prozessmodell entfernt und wird zusätzlich ein direkter Effekt der Ausgangsplatzierung $\left(\mathrm{t}_{1}\right)$ auf die Interaktion $\left(t_{2}\right.$ ) eingeführt, ergibt sich das in Abbildung 6.2.3b wiedergegebene Pfadmodell, das eine bessere Anpassung an die Daten aufweist (Satorra-Bentler Scaled Chi-Quadrat $=14.6312 ; \mathrm{df}=24 ; \mathrm{p}$-value $=0.9311) .{ }^{72}$

Im Vergleich zum Ausgangsmodell fällt die nicht so erwartete beinahe Unabhängigkeit der Interaktion in der Anfangsphase $\left(t_{1}\right)$ von der Ausgangsplatzierung $\left(t_{1}\right)$ auf, scheinbar stehen die Kontakte zu deutschen Personen nicht in direktem Zusammenhang mit der Positionierung auf dem Arbeitsmarkt.

69 Auf ein Verfahren zur Behandlung fehlender Werte wurde verzichtet, da die Ausfallsprozesse im Hinblick auf die Fragestellung möglicherweise als missing not at random aufgefasst werden müssen (vgl. Reinecke 2005, 283-289).

70 Für die Modellschätzung wurde ebenfalls aus Rücksicht auf die niedrige Fallzahl anstellte des Weighted-Least-Squares-Schätzers die robuste Maximum-Likelihood-Schätzung (ML) verwendet.

71 Die Weighted-Least-Squares Schätzung ergab für das Ausgangsmodell eine nicht angemessene Modellanpassung (Chi-Quadrat $=43.31$; $\mathrm{df}=18$; $\mathrm{p}$-value $=0.00$; RMSEA $=0.0995$; -Close $=0.0185$ ).

72 An dieser Stelle bleibt anzumerken, dass die sehr gute Modellanpassung auch im Zusammenhang mit der geringen Fallzahl $(N=143)$ steht und ein Test an einem größeren Datensatz eine härtere Prüfung der Datenanpassung des Modells darstellen würde. 


\section{Abbildung 6.2.3b: Strukturmodell zur Erklärung der Abwanderungs- und Einbürge-}

rungsvorgänge über den Prozess der Sozialintegration

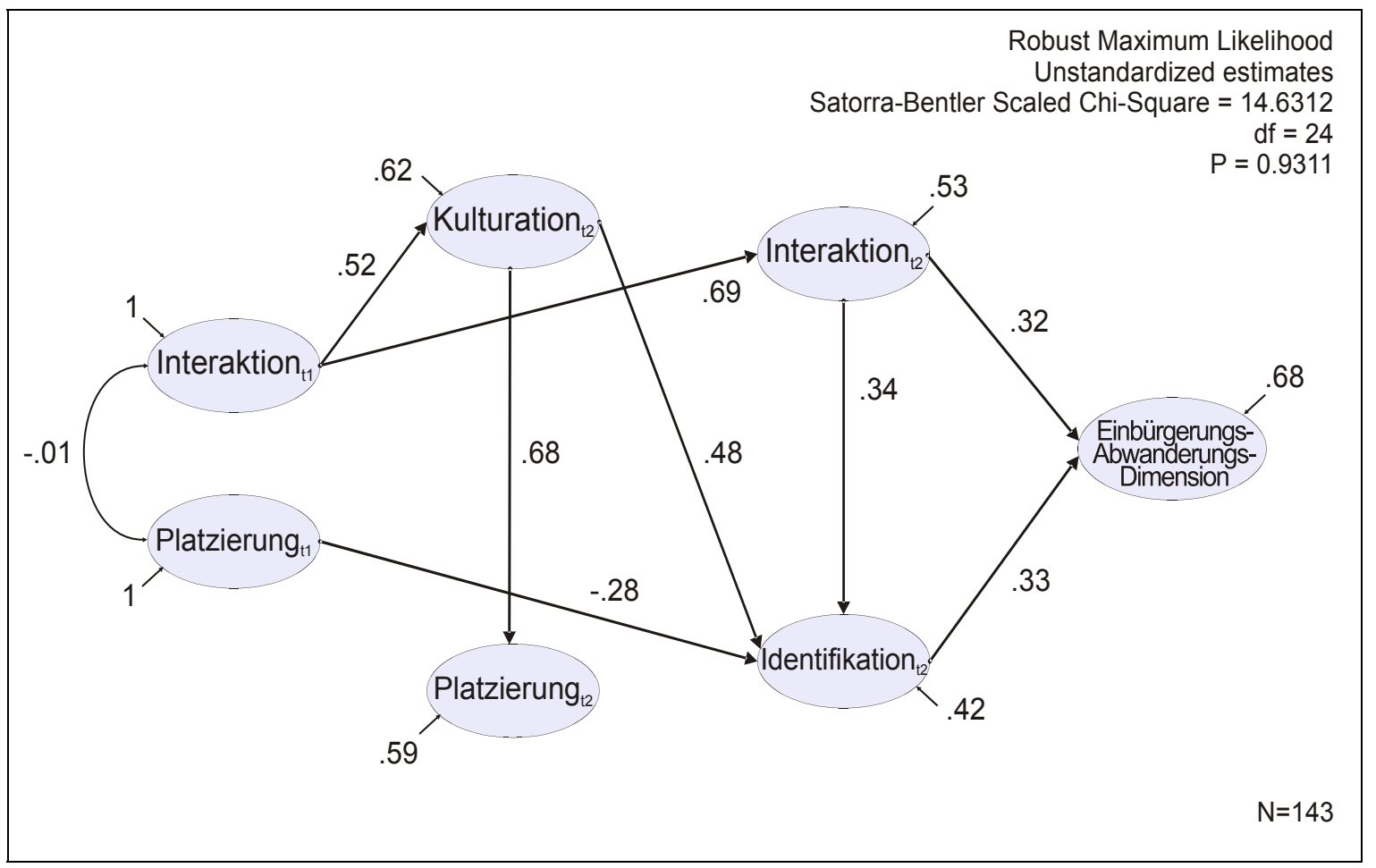

Robuste Maximum Likelihood Schätzung ${ }^{73}$, listwise deletion

Darüber hinaus bestätigt das Ergebnis weiter die geringere Bedeutung der Platzierungen für die Sozialintegration, denn alle anderen erwarteten Wirkungen konnten - mit Ausnahme der direkten Beziehung der ersten auf die spätere Platzierung nicht an den Daten bestätigt werden. Die Platzierung spielt hingegen eine ganz unerwartete Rolle und wirkt im Gefüge des Modells negativ auf Identifikation $\left(t_{2}\right)$ ein. Die Interaktion $\left(t_{1}\right)$ mit deutschen Personen in der Ankunftsphase hingegen erfüllt die theoretischen Erwartungen und fördert sowohl die spätere Interaktion $\left(\mathrm{t}_{2}\right)$ als auch die Kulturation ( $\mathrm{t}_{2}$ ) der Immigranten. Der postulierte und auch so bei den vorherigen Modellen bestätigte Effekt der Interaktion $\left(t_{2}\right)$ auf die Identifikation $\left(t_{2}\right)$ ist auch im Prozessmodell signifikant.

Die große Bedeutung der Kulturation für eine höhere Positionierung auf dem Arbeitsmarkt (.68) und die Identifikation mit der Aufnahmegesellschaft (.48) wird auch

73 Für das modifizierte Modell ergab die Weighted-Least-Squares Schätzung ebenfalls eine gute Übereinstimmung mit den empirischen Daten (Chi-Quadrat $=24.315 ; \mathrm{df}=23 ; \mathrm{p}$-value $=0.444$; $\mathrm{RMSEA}=$ 0.01; P-Close $=0.8156$ ). 
in diesem Modell wieder bestätigt. Allerdings besitzt die Kulturation in diesem Modell keinen direkten Einfluss mehr auf die Interaktion $\left(t_{2}\right)$ und auf die EinbürgerungsAbwanderungs-Dimension. Diese Veränderung im Vergleich zu den oben dargestellten Erklärungsmodellen kann auf den starken Einfluss der ersten gemessenen Interaktion $\left(t_{1}\right)$ auf die beiden Subdimensionen der Sozialintegration zurückgeführt werden. Die Varianz des Auftretens bzw. Ausbleibens von Einbürgerung und Abwanderung wird in dem modifizierten Prozessmodell durch Identifikation (.33) und die Interaktion zu $\mathrm{t}_{2}(.32)$ zu $32 \%$ erklärt.

Das Prozessmodell zeichnet sich insgesamt im Vergleich zum Ausgangsmodell durch eine deutlich geringere Komplexität aus. Überraschend ist die gegenläufige Funktion von Platzierung und Interaktion im Prozess. Während Interaktion durchgängig eine fördernde Wirkung auf die anderen Komponenten der Sozialintegration aufweist, fällt der Effekt der ersten Positionierung auf dem Arbeitsmarkt $\left(t_{1}\right)$ in Richtung der Identifikation negativ aus und fördert somit indirekt die Abwanderung.

Leider stehen in den SOEP-Daten bisher für eine weitergehende Prüfung des Prozesses der Sozialintegration und seiner Wirkung auf die Einbürgerungs- und Abwanderungsvorgänge nicht genügend Fälle zur Verfügung. Es hätte sich ansonsten, vor dem Hintergrund der anderen Analyseergebnisse, eine getrennte Betrachtung der Nationalitätsgruppen empfohlen. Auch besteht bedingt durch die Fallzahl nicht die Möglichkeit, unterschiedliche Einwanderungsphasen oder Einwanderungsgründe zu berücksichtigen, obwohl dies sicherlich zum besseren Verständnis der Prozesse beitragen würde. 


\subsubsection{Zusammenfassung zu den Ergebnissen der kausalen Analysen}

Ausgehend von dem im theoretischen Teil dieser Arbeit explizierten Prozessmodell der Sozialintegration von Immigranten wurde in diesem Kapitel schrittweise das Auftreten bzw. Ausbleiben von Abwanderung und Einbürgerung erklärt.

Zur Umsetzung mit den Daten des Sozio-oekonomischen Panels wurde zu Beginn die Stabilität der kausalen Beziehungen innerhalb des Modells der Sozialintegration geprüft. Die Modellprüfungen ergaben dabei, dass entgegen der theoretischen Erwartungen die Plazierung keinen direkten fördernden Einfluss auf die Identifikation der Befragten besitzt. Im Rahmen der Prüfungen konnte die Stabilität der leicht modifizierten kausalen Struktur über die Zeit und innerhalb der gebildeten Herkunftsgruppen an den Daten bestätigt werden, wenn auch einige Unterschiede in der Bedeutung der einzelnen Subdimensionen der Sozialintegration zu verzeichnen waren. Herauszuheben sind hier über die Zeit das gestiegene Gewicht der Kulturation im Gefüge der Sozialintegration und die für die Gruppe der (Ex-)Jugoslawen im Vergleich größere Wirkung der Platzierung auf den Kontakt zu deutschen Personen.

Für den Test der Sozialintegration als Erklärung der Einbürgerungs- und Abwanderungsvorgänge wurde das bereits zuvor verwendete Ausgangsmodell unter Einbeziehung der Einbürgerungs-Abwanderungs-Dimension auf Basis der Daten des Jahres 1999 spezifiziert. Auch in diesem Fall ist das theoretisch begründete Ausgangsmodell nicht mit den empirischen Daten zu vereinbaren. Im Rahmen der Modifikation konnte ein Erklärungsmodell gefunden werden, das sich an den Gesamtdaten der Jahre 1995 und 1999 bestätigen lässt. Begründet über die in den deskriptiven Analysen festgestellten Unterschiede zwischen den Herkunftsgruppen wurde dieses Modell zur Erklärung weiter verwendet und ergab in den getrennten Analysen eine befriedigende bis gut Modellanpassung an die Daten. Die auch hier vorhandenen Differenzen zwischen den Herkunftsgruppen bestehen wie schon zuvor in der Bedeutung von Platzierung und Kulturation. In der Gruppe der Befragten (ex-) jugoslawischer Herkunft zeigt sich erneut ein stärkere Effekt (.391) der Platzierung auf die Interaktion, der im Falle der Personen türkischer Herkunft mit .114 sehr 
schwach ausfällt. In Bezug auf die Erklärung der Vorgänge ist insbesondere die Kulturation von herausgehobener Bedeutung, wenn auch bei der betrachteten Gruppe der EU-Bürger ihre Wirkung indirekt über die Identifikation verläuft. Insgesamt können mit Hilfe der Sozialintegration, jedoch nicht ohne Abstriche im Hinblick auf die postulierten Beziehungen, die Einbürgerungs- und Abwanderungsvorgänge erklärt werden. Es zeigte sich, dass der Erwerb kultureller Kenntnisse gemessen über die Deutschkenntnisse und letztlich die Identifikation mit der Aufnahmegesellschaft für den weiteren Verbleib und die Absicht deutscher Staatsbürger zu werden ausschlaggebend sind. In dieser Hinsicht haben Interaktion durchgängig und Platzierung mal mehr mal weniger nur eine indirekte Funktion. Wie sich darüber hinaus im herkunftsbezogenen Vergleich zeigte, kann die Varianz der Einbürgerungs-Abwanderungs-Dimension im Modell der türkischen Teilgruppe schlechter als bei den beiden anderen Nationalitätengruppen erklärt werden, was als Indiz für eine stärkere Einbindung über die ethnische Community in der Bundesrepublik interpretiert werden kann.

Die Anwendung des Prozessmodells der Sozialintegration auf die Daten des Sozio-oekonomischen Panels wurde geprägt durch die für den geplanten Test geringe Fallzahl an Personen in der Zielgruppe. Beim Test des Ausgangsmodells zeigte sich, ebenso wie in den vorangegangen Modellprüfungen, dass nicht alle postulierten Beziehungen als wesentlich im Sinne von statistischer Signifikanz angesehen werden können. Während für Interaktion, Kulturation und Identifikation die erwartete positiv verstärkende Rolle im Modell bestätigt werden konnte, war das Ergebnis zur Wirkung der Platzierung im Prozess der Sozialintegration eher überraschend. Unter Berücksichtigung der Ausgangsplatzierung $\left(t_{1}\right)$ zeigte sich ein erheblicher negativer Effekt von Platzierung auf Identifikation. Ähnlich wie in einigen Forschungsergebnissen zur Bildung im Rahmen der Remigrations- und Naturalisierungsforschung (Reagan/Olson 2000; Yang 2002; Nekby 2004; Diehl/Preisendörfer 2006) wirkt bessere Positionierung auf dem Arbeitsmarkt nicht konsistent in einer Verstärkung der Bindung zur Aufnahmegesellschaft, denn je höher die Positionierung am Anfang des Aufenthaltes ausfällt, umso niedriger sind die Identifikation und umso seltener kommt es zu Einbürgerungen. Höhere berufliche Positionen sind offensichtlich im Zusammenhang mit Abwanderung eher als generalisierbares Humankapital und damit als Potential für eine erneute Emigration zu interpretieren. 
Der von der Theorie her postulierte Effekt einer Einbindung durch Platzierung kann mit den vorliegenden Daten nicht bestätigt werden.

Insgesamt lassen sich die Ergebnisse dahingehend zusammenfassen, dass mit dem Modell der Sozialintegration die Abwanderungs- und Einbürgerungsvorgänge erklärt werden können, wenn auch einige Abweichungen von den postulierten Kausalbeziehungen zu verzeichnen sind. Insbesondere die Unterschiede in den Herkunftsgruppen und die nicht bindende Wirkung der über die Positionierung auf dem Arbeitsmarkt gemessenen Platzierung im Hinblick auf die Einbürgerungs-Abwanderungs-Dimension sind hier zu nennen.

Inwieweit die Ergebnisse durch Verzerrungen beeinträchtigt sind, kann letztlich nicht exakt abgeschätzt werden, da beispielsweise keine Kontrollstichproben zur Prüfung auf Unterschiede existieren. Die Übereinstimmungen mit den Erwartungen und in Teilbereichen mit den Ergebnissen anderer Forschungsarbeiten kann als Hinweis auf nicht allzu dramatische Verzerrungen gewertet werden. Bezogen auf den Test des Prozessmodells ist allerdings sehr große Vorsicht anzuraten, da eine Basis von 143 Fällen als sehr gering und damit als besonders anfällig gegenüber Verzerrungen angesehen werden muss. In diesem Punkt sind die Ergebnisse mehr als Indizien für die gefundene Kausalstruktur zu interpretieren, die eine Überprüfung an geeigneteren Daten erforderlich machen. 


\section{Zusammenfassung und Diskussion}

Integration ist eine Herausforderung, mit der sich sowohl Immigranten, deren Nachkommen als auch die Mitglieder der Aufnahmegesellschaft konfrontiert sehen. Eingelagert in eine Mehrheits-Minderheitsrelation, bei der die Immigranten in der Regel die Minderheit stellen, reagieren die Akteure auf die durch Einwanderung veränderten Lebensumstände. In Abhängigkeit des Größenverhältnisses von Majorität und Minorität ist im Zuge von Einwanderungsprozessen auf beiden Seiten mit mehr oder weniger tiefgreifenden Veränderungen zu rechnen. Inwieweit Integration erfolgreich verläuft oder aber misslingt, ist von vielen Faktoren abhängig. Aber gezielte politische Maßnahmen, die am Prozess der Integration ansetzen, können dazu beitragen, die Integrationspotentiale besser zu entwickeln und helfen die erforderlichen Transaktionskosten zu verringern. Allerdings müssen dazu die Strukturen der Prozesse bekannt sein, ansonsten fehlt es den Programmen, so wie vom Sachverständigenrat für Zuwanderung und Integration kritisiert, an Effektivität und Zielgenauigkeit (vgl. Sachverständigenrat für Zuwanderung und Integration 2004, 434). Ausgehend von der Frage, ob das Auftreten oder Ausbleiben von Einbürgerung und Abwanderung über die Integration in die Aufenthalts- bzw. Mehrheitsgesellschaft erklärt werden kann, sollte mit dieser Arbeit ein Beitrag zur Klärung der kausalen Struktur von Integrationsprozessen geleistet werden.

Im Sinne der Fragestellung werden Einbürgerung und Abwanderung als Pole des Integrationsverlaufs interpretiert. Einbürgerung steht insofern für eine dauerhafte Bleibeabsicht des Immigranten bei gleichzeitiger Anerkennung durch die Aufnahmegesellschaft, wo hingegen in Abwanderung die Auflösung der im Zuge des Integrationsprozesses entstandenen Bindungen gesehen werden kann. Vor dem Hintergrund der Hauptlinien sozialwissenschaftlicher Auseinandersetzung mit Integrationsverläufen und Anpassungsmustern von Immigranten und auf Basis charakteristischer Gemeinsamkeiten von Abwanderung und Einbürgerung wurde der Ansatz von Hartmut Esser zur Sozialintegration für die Erklärung der Abwanderungs- und Einbürgerungsvorgänge ausgewählt.

Esser definiert Sozialintegration als Einbindung der Individuen und Gruppen in eine Gesellschaft. Um die Breite des Integrationsgeschehens abbilden zu können, 
ist die Sozialintegration in die Teilbereiche Kulturation, Platzierung, Interaktion und Identifikation untergliedert. Sozialintegration von Immigranten kann sich dem Modell nach sowohl auf die Mehrheitsgesellschaft, als auch auf eine ethnische Gemeinde oder aber auf die Herkunftsgesellschaft beziehen. Ist die Sozialintegration in jeder Hinsicht auf die Mehrheitsgesellschaft bezogen, so entspricht dies einer vollständigen Assimilation des Immigranten. Analog zur Sozialintegration lassen sich demnach auch für die Assimilation kulturelle, strukturelle, soziale und identifikative Subdimensionen unterscheiden. Die theoretischen Gemeinsamkeiten von Sozialintegration und Assimilation ermöglichen es, für die genauere Bestimmung der kausalen Beziehungen zwischen den Subdimensionen auf ein bereits 1980 von Esser vorgestelltes Prozessmodell der Assimilation zurückzugreifen. Ausgehend von diesem im Sinne einer Assimilations-Dissimilations-Perspektive interpretierten Prozessmodell wurden im empirischen Teil über Kulturation, Platzierung, Interaktion und Identifikation der Immigranten das Auftreten bzw. Ausbleiben der Einbürgerungs- und Abwanderungsvorgänge erklärt.

Nachdem im theoretischen Teil die Beziehungen der Subdimensionen untereinander und zu den Einbürgerungs- und Abwanderungsvorgängen expliziert wurden, konnte in den statistischen Analysen der Frage nachgegangen werden, ob die erwarteten Zusammenhänge sich an empirischen Daten bestätigen lassen. Die Analysen basieren auf den Daten des Sozio-oekonomischen Panels des Deutschen Instituts für Wirtschaftsforschung. In einem Zwischenschritt war zunächst geklärt worden, inwieweit in den SOEP-Daten geeignete Indikatoren für die einzelnen Konstrukte der Theorie vorhanden sind und welches statistische Analysemodell bei gegebener Datenstruktur zum Einsatz kommen sollte. Obwohl nicht in allen Fällen die assimilativ-dissimilative Perspektive in den Operationalisierungen wiederzufinden ist, erlauben es die Paneldaten doch, die Kausalstruktur des Prozessmodells der Sozialintegration einer empirischen Prüfung zu unterziehen. Mit linearen Strukturgleichungsmodellen steht auch ein geeignetes statistisches Verfahren für die Prüfung der kausalen Beziehungen der Konstrukte zur Verfügung.

Vor den eigentlichen Modelltests wurden zunächst deskriptive Analysen durchgeführt, die hinsichtlich des Vergleichs der Einwanderergeneration mit ihren Nachkommen ergaben, dass die jüngere Generation sich bisher deutlich besser integrie- 
ren konnte und auch eine geringere Tendenz zur Abwanderung zeigte als die Angehörigen der ersten Generation. Die Unterschiede im Hinblick auf die Einbürgerung waren zwar nicht ganz so deutlich ausgeprägt, aber die Einwanderer weisen gemäß der geringeren Sozialintegration auch eine geringere und damit erwartungskonforme Einbürgerungsrate auf.

Ein Vergleich der Sozialintegration nach ausgewählten Herkunftsgruppen im Verhältnis zu Abwanderung und Einbürgerung zeigte hingegen eine klare Diskrepanz zwischen Daten und den erwarteten Zusammenhängen. Obwohl Personen aus den EU-Mitgliedstaaten und ihre Nachkommen im Durchschnitt stärkere Sozialintegrationswerte aufwiesen als Personen türkischer und (ex-)jugoslawischer Herkunft, sind die Abwanderungsraten der EU-Bürger höher und die Einbürgerungsraten geringer als bei den Vergleichsgruppen. Diese Gruppenunterschiede wurden zum Anlass genommen, im Rahmen der Modellprüfungen die Stabilität der Modellannahmen auch nach Herkunftsgruppen zu prüfen.

Der eigentliche Modelltest erfolgte in mehreren Schritten, wobei zunächst die kausale Struktur der Sozialintegration geprüft wurde. In diesem ersten Prüfungsschritt konnte die erwartete Kausalstruktur weitgehend bestätigt werden, allerdings erwies sich der direkte Effekt der Platzierung auf die Identifikation als nicht signifikant. Insgesamt zeigten die Tests eine über die Jahre zunehmende Bedeutung der Kulturation im Gefüge der Sozialintegration. Darüber hinaus waren zwischen den Herkunftsgruppen kleinere Differenzen zu verzeichnen. Daran anschließend wurde das ermittelte Modell zur Erklärung der Einbürgerungs- und Abwanderungsvorgänge eingesetzt. Es zeigte sich dabei, dass Kulturation gemessen über die subjektiven Deutschkenntnisse und die Identifikation mit der Mehrheitsgesellschaft direkt auf den weiteren Verbleib in der Bundesrepublik und die Absicht deutscher Staatsbürger zu werden, einwirken. Auch hier bestätigte sich über alle Herkunftsgruppen hinweg die starke Bedeutung der Kulturation im Gefüge der Sozialintegration. Allerdings erwies sich die Sozialintegration nicht durchgängig als erklärungskräftig. Im Falle der türkischen Befragten konnte nur sehr wenig der vorhandenen Varianz bezogen auf die Einbürgerungs- und Abwanderungsvorgänge erklärt werden. 
Im letzten Prüfungsschritt wurde dann das Prozessmodell der Sozialintegration zur Erklärung der betrachteten Vorgänge verwendet. Die Verallgemeinerbarkeit dieser Ergebnisse ist jedoch vermutlich durch die geringe Zahl an Fällen, die für den Modelltest zu Verfügung standen, erheblich beeinträchtigt. Im Unterschied zu den zuvor spezifizierten Modellen, wurden neben der im Verlauf der Jahre erreichten Sozialintegration die erste Platzierung auf dem Arbeitsmarkt und die Interaktion zu Beginn des Aufenthalts in der Bundesrepublik im Modell berücksichtigt. Erwartungsgemäß beeinflusst der erste Kontakt zu deutschen Personen die Kulturation und auch die spätere Interaktion. Die in der Anfangsphase erreichte Platzierung hingegen besitzt nur eine direkte Wirkung auf die Identifikation, der zufolge eine zu Beginn höhere Positionierung auf dem Arbeitsmarkt eine geringere Identifikation mit der Aufnahmegesellschaft nach sich zieht. Die erwarteten Effekte der Platzierung auf die Kulturation und die spätere Positionierung konnte nicht an den Daten bestätigt werden. Bei diesem Modell haben letztlich die im Verlaufe der Jahre erreichte Identifikation und die zuletzt gemessene Interaktion eine direkte Wirkung auf die Einbürgerungs- und Abwanderungsvorgänge, indirekt üben noch die Kulturation und der zu Beginn des Aufenthalts vorhandene Kontakt einen Einfluss aus.

Wie sind die Ergebnisse der empirischen Analysen nun im Hinblick auf die Anwendbarkeit des Sozialintegrationsansatzes auf die Bindung von Immigranten an die Aufnahmegesellschaft zu bewerten und welche Konsequenzen lassen sich daraus ableiten? Im Rahmen dieser Fragen ist auch auf Problempunkte einzugehen, die bisher nur am Rande angesprochen worden sind. 
Orientiert an den Forschungsergebnissen zu den Determinanten der Einbürgerung und Abwanderung wurde die These abgeleitet, dass Zuwanderer, denen es gelingt, vielfältige Bindungen zur Aufnahmegesellschaft aufzubauen, mit höherer Wahrscheinlichkeit längerfristig in der neuen Heimat bleiben und auch eher durch Einbürgerung eine Gleichstellung erfahren. Die Ergebnisse der Datenanalysen lassen sich als Bestätigung dieser sehr allgemein gehaltenen Hypothese über die Zusammenhänge von Integration und Bindung deuten. Im Hinblick auf das verwendete Modell der Sozialintegration ist aber eine differenziertere Antwort notwendig und auch möglich. In den Analysen zeichnet sich fast durchgängig eine ausgeprägte Zweiteilung der Modelle in einen stabileren Teil, der auch erwartungskonform ausfällt und in einen weit weniger eindeutigen Teil $a b$.

Die Strukturgleichungsmodelle ebenso wie die Analysen der Mittelwerte ergaben hinsichtlich der Zusammenhänge von Kulturation, Interaktion und Identifikation ein relativ konsistentes Bild. Der Erwerb von kulturellen Kenntnissen, gemessen über die subjektive Deutschkompetenz, hat erheblichen Einfluss auf die Kontakte zu Angehörigen der Mehrheitsgesellschaft; diese beiden Faktoren zusammen bestimmen in hohem Maße die Identifikation mit der Aufnahmegesellschaft. Ganz besonders ist dabei die erwartungsgemäß starke Rolle der Kulturation hervorzuheben. Die Kulturation muss in diesem Zusammenhang zum einen als Ressource im Sinne von Humankapital gesehen werden, das als Basis für Interaktion erworben werden muss und zum anderen ist speziell die Sprache ein Medium, deren Gebrauch eine Rückwirkung auf die Identität zu besitzen scheint: Ein Ergebnis, das frühere Untersuchungen zur kausalen Struktur dieser Integrationsprozesse bestätigt (Esser 1981). Menschen mit Migrationshintergrund, denen es gelingt, sich die in einer Aufnahmegesellschaft erforderlichen kulturellen Kenntnisse anzueignen und soziale Beziehungen zur autochthonen Bevölkerung aufbauen, entwickeln eher eine emotionale Bindung zur Mehrheitsgesellschaft. Mit der Kulturation, Interaktion und Identifikation scheinen die Bindungen einherzugehen, über die zumindest teilweise eine Erklärung der Einbürgerungs- und Abwanderungsvorgänge möglich ist.

Dabei ist die Funktion der Interaktion in diesem Gefüge nicht zu unterschätzen. Speziell das Prozessmodell weist daraufhin, dass dem ersten Kontakt zu deutschen Personen für den weiteren Integrationsverlauf erhebliche Bedeutung zukommt. Re- 
gelmäßiger Kontakt kann als Ausdruck unmittelbarer sozialer Anerkennung interpretiert werden und stellt damit im Sinne Lindenbergs ein grundlegendes Ziel menschlichen Handelns dar. Wenn darüber hinausgehend die Ergebnisse der Vorurteilsforschung in Rechnung gestellt werden, ist die Interaktion neben der Förderung des Spracherwerbs ein wichtiger integrationspolitischer Ansatzpunkt, denn intensiver Kontakt wirkt gleichzeitig auch reduzierend auf Vorurteile und distanzierende Verhaltensabsichten (vgl. Wagner/van Dick/Endrikat 2002; Leibold/Kühnel 2006). Allerdings sind die Ergebnisse nicht in allen Punkten so eindeutig und theoriekonform wie im Hinblick auf die kulturellen, sozialen und identifikativen Aspekte des Modells.

Bereits die Mittelwertanalysen ließen im Falle der Platzierung tendenzielle Unterschiede im Vergleich zu den anderen Komponenten der Sozialintegration erkennen. Die durchschnittliche Positionierung auf dem Arbeitsmarkt zeichnete sich im Verlauf der Jahre nicht durch einen stetigen Anstieg aus wie bei den übrigen Subdimensionen der Sozialintegration, sondern zeigte in fast allen Teilgruppen ein Maximum im Jahr 1991. Dieser Höhepunkt dürfte auf die im Vergleich niedrigen Arbeitslosenzahlen in der Zeit nach der Wiedervereinigung zurückzuführen sein.

Ausgehend von den theoretischen Erwartungen ist festzustellen, dass die Platzierung innerhalb des Modells der Sozialintegration nicht die ihr zugedachte Funktion erfüllte. Während Esser der Platzierung eine Schlüsselfunktion für jede nachhaltige Sozialintegration zuspricht, konnte dies an den Daten des Sozio-oekonomischen Panels nicht bestätigt werden. Allein der erwartete direkte Einfluss der Kulturation auf die Platzierung kann über die Zeit und in allen Teilgruppen als stabil angesehen werden, was einmal mehr die Bedeutung der Kulturation für die Sozialintegration der Immigranten unterstreicht. Für die im Verlauf der Jahre erzielte Positionierung auf dem Arbeitsmarkt war kein direkter aber ein leichter nicht immer stabiler fördernder Einfluss über die Interaktion auf die emotionale Bindung zu verzeichnen. Zwischen den getrennt betrachteten Herkunftsgruppen ergaben sich in dieser Hinsicht bemerkenswerte Differenzen, während bei den Personen (ex-)jugoslawischer Herkunft der Effekt am deutlichsten ausgeprägt war, hatte er für die Sozialintegration der türkischen Teilgruppe sehr viel geringere Bedeutung. Im Hinblick auf die zu Beginn des Aufenthalts erzielte Platzierung ergab sich sogar ein negativer Effekt 
auf die Identifikation, was indirekt einer tendenziell abwanderungsfördernden Wirkung entspricht. Allerdings beruht dieses Ergebnis auf einer sehr kleinen Auswahl an Immigranten, für die die notwendigen Informationen zur Verfügung standen. Diese uneindeutige Rolle der Platzierung im Rahmen der Sozialintegration legt eine heterogene Struktur der Daten nahe. Oder anders formuliert: Das Modell der Sozialintegration ist in diesem Punkt nicht mit den Daten zu vereinbaren.

Dieses Ergebnis korrespondiert mit der für Schweden berichteten nicht-linearen Beziehung zwischen ökonomischer Einbindung und Abwanderung (vgl. Nekby 2004). Es ist davon auszugehen, dass für Immigranten mit schlechter bis mittlerer Positionierung ein bindender Effekt bestätigt werden kann, für höher und hoch Qualifizierte kann allerdings analog zur Bildung eine entgegengesetzte Wirkung vermutet werden (vgl. Diehl/Preisendörfer 2006).

Die Bindung an die Aufnahmegesellschaft über Positionierung scheint, insbesondere bei den Niederlassungsfreiheit genießenden EU-Binnenmigranten, nicht ausschließlich auf den nationalen Arbeitsmarkt bezogen zu sein, sondern spielt sich offensichtlich in Abhängigkeit vom Qualifikationsniveau zunehmend im transnationalen oder zumindest binationalen Raum ab. Hierin könnte auch eine Begründung für die geringere bindende Wirkung der im Vergleich hohen Sozialintegration bei EU-Bürgern liegen. Mit der verwendeten fast rein assimilationsbezogenen Operationalisierung der Platzierung bleibt dieser Aspekt unberücksichtigt. Um aber besser die Wirkung der Platzierung auf die Bindung von Immigranten und ihrer Nachkommen an die Aufnahmegesellschaft einschätzen zu können, ist dieser Teil des Modells der Sozialintegration unverzichtbar. Leider sind in den Daten des Sozio-oekonomischen Panels bisher keine adäquaten Indikatoren für die Messung dieses transnationalen bzw. dissimilativen Aspekts des Arbeitsmarktbezuges vorhanden. ${ }^{74}$

Ein ähnlicher, wenn auch in seiner Richtung umgekehrter Zusammenhang, ist im Hinblick auf die Bindung der Personen türkischer Herkunft an die deutsche Aufnahmegesellschaft zu vermuten. Obwohl die türkische Teilgruppe durchgehend gerin-

74 An dieser Stelle bleibt noch anzumerken, dass die referierten Befunde kein Argument darstellen, die integrationspolitische Zielsetzung einer Vermeidung ethnischer Schichtungen weniger Bedeutung zukommen zu lassen. Schichtungsverhältnisse mit ethnischer Prägung stellen immer ein leicht zu mobilisierendes Konfliktpotential in einer Gesellschaft dar. 
gere Sozialintegrationswerte aufweist, sind im Vergleich zu den deutlich besser integrierten EU-Bürgern relativ mehr Einbürgerungen und weniger Abwanderungen zu verzeichnen. Gleichzeitig können, trotz der guten Übereinstimmung des Modells mit den empirischen Daten, über die Sozialintegration nur in sehr geringem Umfang die Einbürgerungs- und Abwanderungsvorgänge der Personen türkischer Herkunft prognostiziert werden. Im Sinne der Assimilations-Dissimilations-Perspektive können diese Befunde als Ausdruck für die Bindungskraft einer starken ethnischen Gemeinschaft interpretiert werden. Wenn Immigranten durch die Vollständigkeit einer ethnischen Kolonie in die Lage versetzt werden, unter Verzicht auf assimilative Handlungen ihre Bedürfnisse zu befriedigen, dann entstehen Bindungen, die vermutlich Remigration als weniger attraktiv erscheinen lassen. Speziell die Einbindung über den ethnischen Arbeitsmarkt und die gruppeninternen Sozialkontakte dürften in diesem Zusammenhang von herausgehobener Bedeutung sein. Dies würde die von Elwert entwickelte These der gesellschaftlichen Integration durch Binnenintegration in einer ethnischen Kolonie stützen (vgl. Elwert 1982). Hinweise hierfür finden sich auch in den in den Untersuchungen von Yang und Dornis, die eine signifikant fördernde Wirkung von Konzentration und Größe der ethnischen Gemeinschaft auf die Einbürgerung feststellen konnten (vgl. Yang 1994, Dornis 2001).

Sowohl die widersprüchlichen Befunde zur Platzierung, als auch die Indizien für eine Binnenintegration der Personen türkischer Herkunft deuten auf ein stärkeres Gewicht der dissimilativen Komponenten innerhalb der Sozialintegration hin. Transnationale Bezüge und die ethnisch-kulturelle Pluralisierung scheinen insbesondere im Hinblick auf die Einbürgerungs- und Abwanderungsvorgänge von größerer Bedeutung zu sein. In diesem Zusammenhang ist die Offenheit gegenüber dem Ergebnis einer der Vorzüge des Modells der Sozialintegration, denn neben assimilativen Aspekten ist es auch möglich, dissimilative Entwicklungen zu berücksichtigen. Wobei es hierfür nicht unwesentlich zu sein scheint, gleichzeitig den nationalen wie den transnationalen Bezug in das Modell zu integrieren. Die stärkere Berücksichtigung der dissimilativen Aspekte im Rahmen der Sozialintegration könnte m. E. die Fruchtbarkeit und Erklärungskraft des Ansatzes deutlich erhöhen.

Insgesamt konnten mit den empirischen Analysen die erwarteten Beziehungen von Kulturation, Interaktion und Identifikation als weitgehend stabil bestätigt wer- 
den. Gleichzeitig sind die Ergebnisse als starker Beleg für die zentrale Funktion der sprachlichen Assimilation für die Sozialintegration von Immigranten zu werten. Die im Kontext der aufgetretenen Probleme angeführten Argumente für eine konsequente Erweiterung des Sozialintegrationsansatzes um dissimilative Aspekte stehen allerdings in Anbetracht der mehrfach angesprochenen Datenprobleme auf nicht allzu sicheren Beinen. Auf Basis der Daten des Sozio-oekonomischen Panels sind den Analysen, bedingt durch Stichprobenzusammensetzung, Ausgangsstichprobengröße und Ausfallprozesse, in Bezug auf den zeitlichen Horizont wie auch der eingehenderen Betrachtung von Subgruppen Grenzen gesetzt. So kommt auch Sonja Haug in einer aktuellen Übersicht zur Datenlage in der Migrations- und Integrationsforschung zu dem Urteil, dass die zur Verfügung stehenden Datenquellen als nicht ausreichend einzustufen sind (vgl. Haug 2005). Ein Integrations-Panel für Immigranten und ihre Nachkommen wie es der Sachverständigenrat für Zuwanderung und Integration empfiehlt, ist daher für jede tiefergehende Analyse der Integrationsverläufe erforderlich (vgl. Sachverständigenrat für Zuwanderung und Integration 2004, 425). Im Rahmen eines solchen Panels wäre es darüber hinaus sinnvoll, die Evaluation integrationspolitischer Maßnahmen von Beginn an im Design zu verankern. 


\section{Literatur}

Alba, R. (2004), Language assimilation today: bilingualism persists more than in the past, but English still dominates. CCIS Working Paper, Nr. 111, San Diego.

Alba, R. und Nee, V. (2004), Assimilation und Einwanderung in den USA. IMIS-Beiträge, Heft 23: S. 21-60.

Alpheis, H. (1990), Erschwert die ethnische Konzentration die Eingliederung?, S. 147-184, in: H. Esser und J. Friedrichs (Hrsg.), Generation und Identität, Opladen.

Alvarez, R. (1987), A Profile of the Citizenship Process among Hispanics in the United States. International Migration Review Jg. 21: S.327-351.

Andreß, H.-J., Hagenaars, J. A. und Kühnel, S. (1997), Analyse von Tabellen und kategorialen Daten, Heidelberg.

Angenendt, S. (1992), Ausländerforschung in Frankreich und der Bundesrepublik Gesellschaftliche Rahmenbedingungen und inhaltliche Entwicklung eines aktuellen Forschungsbereiches. Frankfurt/Main.

Anthias, F. und Yuval-Davis, N. (1992), Racialized Boundaries - Race, Nation, Gender, Colour and Class and the Anti-racist Struggle, London.

Bade, K. J. (2002), Die „Festung Europa“ und die illegale Migration, 25-35, in: M. Blum, A. Hölscher und R. Kampling (Hrsg.): Die Grenzgänger, Opladen.

Bade, K. J. und Bommes, M. (2004), Einleitung. IMIS-Beiträge, Heft 23: S. 7-20.

Baringhorst, S. (1991), Fremde in der Stadt - Multikulturelle Minderheitenpolitik, dargestellt am Beispiel der nordenglischen Stadt Bradford, Baden-Baden.

Baringhorst, S. (2003), Australia - the Lucky Country? Multikulturalismus und Migrationspolitik im Zeichen neokonservativer Reformen. Aus Politik und Zeitgeschichte, Heft 26: S. 12-18.

Beauftragte der Bundesregierung für Migration, Flüchtlinge und Integration (2003), Migrationsbericht der Beauftragten der Bundesregierung für Migration, Flüchtlinge und Integration im Auftrag der Bundesregierung, Berlin. 
Becker, G.S. (1975), Human Capital, New York.

Beijbom, U. (1971), Swedes in Chicago: A Demographic an Social Study of the 1846-1880 Immigration, Chicago.

Bernard, W. S., (1936), Cultural Determinations of Naturalisation. American Sociological Review, Jg. 1: S. 943-953.

Bielefeld, U. (1998), Das Konzept des Fremden und die Wirklichkeit des Imaginären, S. 97-128, in: U. Bielefeld (Hrsg.), Das Eigene und das Fremde - Neuer Rassismus in der Alten Welt?, Hamburg.

Bischoff, D. und Teubner, W. (1991), Zwischen Einbürgerung und Rückkehr - Ausländerpolitik und Ausländerrecht der Bundesrepublik Deutschland, Berlin.

Bloemraad, I. (2003), Who Claims Dual Citizenship? The Limits of Postnationalism, the Possibilities of Transnationalism, and the Persistence of Traditionalism. International Migration Review Jg. 38: 389-426.

Blossfeld, H.-P, Hamerle, A. und Mayer, K. U. (1986), Ereignisanalyse - Statistische Theorie und Anwendung in den Wirtschafts- und Sozialwissenschaften, Frankfurt/Main.

Böcker, A. und Thränhardt, D. (2003), Erfolge und Misserfolge der Integration Deutschland und die Niederlande im Vergleich. Aus Politik und Zeitgeschichte, Heft 26: S. 3-11.

Borjas, G. J. (1989), Immigrant and Emigrant Earnings: A Longitudinal Study. Economic Inquiry Jg. 27: S. 21-37.

Borjas, G. J. und Bratsberg, B. (1996), Who leaves? The Outmigration of the Foreign-Born. The Review of Economics and Statistics, Jg. 78: S. 165-176.

Bös, M. (1995), Migranten und soziale Ungleichheit: Soziale und politische SchlieBungen in westlichen Industrieländern, S. 17-24, in W. Seifert (Hrsg.), Wie Migranten leben. Lebensbedingungen und soziale Lage der ausländischen Bevölkerung in der Bundesrepublik. Arbeitspapier FS III 95-401, Berlin.

Brecht, B. (1994), Remigration der Gastarbeiter: Statistische Modellierung von Rückkehrprozessen, Konstanz. 
Breitenbach, B. v. (1982), Italiener und Spanier als Arbeitnehmer in der Bundesrepublik Deutschland, eine vergleichende Untersuchung zur europäischen Arbeitsmigration, München.

Brubaker, R. (1992), Citizenship and Nationhood in France and Germany, Camebridge.

Brubaker, R. (2001), The Return of Assimilation? Changing Perspectives on Immigration and its Sequels in France, Germany and the United States. Ethnic and Racial Studies, Jg. 24: S. 531-548.

Brücker, H. und Kohlhaas, M. (2004), Möglichkeiten der quantitativen und qualitativen Ermittlung von Zuwanderungsbedarf in Teilarbeitsmärkten in Deutschland Eine Analyse der Effekte der Migration in heterogenen Arbeitsmärkten, Expertise für den Sachverständigenrat für Zuwanderung und Integration, Berlin.

Bultmann, P. F. (1999), Lokale Gerechtigkeit im Einbürgerungsrecht, Berlin.

Castel, R. (1996), Nicht Exklusion, sondern Desaffiliation. Ein Gespräch mit Francoise Ewald. Das Argument, Nr. 217: S. 775-780.

Castles, S. (1987): Migration und Rassismus in Westeuropa, Berlin.

Castles, S. (1994), Democracy and Multicultural Citizenship. Australian Debates and their Relevance for Western Europe, S. 3-27, in: R. Bauböck (Hrsg.), From Aliens to Citizens - Redefining the Status of Immigrants in Europe, Aldershot.

Castles, S. (1998), Einwanderung und Sozialpolitik in Australien, S. 171-197, in : M. Bommes und J. Halfmann (Hrsg.), Migration in nationalen Wohlfahrtsstaaten. Theoretische und vergleichende Untersuchungen, IMIS-Schriften, Bd. 6, Osnabrück.

Chiswick, B. R. (1978), The effect of americanization on the earnings of foreignborn men. The Journal of Political Economy, Jg. 86: S. 897-921.

Cinar, D. (1994), From aliens to citizens: a comparative analysis of rules of transition, S. 49-72, in: R. Bauböck (Hrsg.), from Aliens To Citizens: Redefining the State of Immigrants in Europe, Aldershot.

Cohn-Bendit, D. und Schmid, T. (1992), Heimat Babylon. Das Wagnis der multikulturellen Demokratie, Hamburg. 
Constant, A. und Massey, D. S. (2002), Return Migration by German Guestworkers: Neoklassical versus New Economic Theories. International Migration, Jg. 40: S. 5-38.

Constant, A. und Zimmermann, K. F. (2003), The dynamics of repeat migration: a Markov chain analysis. IZA Discussion Paper Nr. 885, Institute for the Study of Labor, Bonn.

DeSipio, L. (1987), Social Science Literature and the Naturalisation Process. International Migration Review, Jg. 21: S. 390-405.

Diehl, C. ( 2002), Wer wird Deutsche/r und warum? Bestimmungsfaktoren der Einbürgerung türkisch- und italienischstämmiger junger Erwachsener - Ergebnisse des Integrationssurveys des BiB. Zeitschrift für Bevölkerungswissenschaft, Jg. 27: S. 285-312.

Diehl, C. und Blohm, M. (2003), Reights or Identity? Naturalization Processes among „Labor Migrants“ in Germany. International Migration Review, Jg. 37: S. 133-162.

Diehl, C. und Preisendörfer, P. (2006), Bedeutung und Bestimmungsfaktoren der Bleibeabsicht Ausländischer Neuzuwanderer. Unveröffentlichtes Manuskript.

Dornis, C. (2001), Einbürgerung in Deutschland. Ihre Rolle bei der Integration von Zuwanderern und die Verwaltungspraxis im Regionalvergleich, Aachen.

Duleep, H. O. (1994), Social Security and the Emigration of Immigrants“. Social Security Bulletin, Jg. 57: S. 37-52.

Dustmann, C. (1993), Earnings adjustment of temporary migrants. Journal of Population Economics, Jg. 6: S. 153-168.

Dustmann, C. (2000) Temporary Migration and Economic Assimilation, in: IZA Discussion Papers Series, Nr. 186.

Dustmann, C. (2002) Arbeitsmarktverhalten, Integration und Rückkehr von Einwanderern. Vierteljahrshefte zur Wirtschaftsforschung; Jg. 71: S. 272-285.

Dustmann, C. (2003), Return Migration, Wage Differential, and the Optimal Migration Duration. European Economic Review, Jg. 47: S.353-369. 
Edin, P.-A., Lalonde, R. J. und Aslund, O. (2000), Emigration of Immigrants and Measures of Immigrant Assimilation: Evidence from Sweden, in: Uppsala University, Department of Economics, Working Paper Series, Nr. 13.

Edwards, W. (1955), The Prediction of Decisions Among Bets. Journal of Experimental Psychology, Jg. 50: S. 201-214.

Edwards, W. (1992), Utility theories: Measurements ans Applications, Boston.

Eisenstadt, S. N. (1954), The Absorption of Immigrants. A comparative study based mainly on the Jewish community in Palestine and the State of Israel, London.

Eitenmüller, S., Gramke, K. und Jens, S. (2001), Zuwanderung und Wohlstandswirkung, Endbericht für die unabhängige Kommission „Zuwanderung“, Basel.

Elwert, G. (1982), Probleme der Ausländerintegration: Gesellschaftliche Integration durch Binnenintegration?, in: Kölner Zeitschrift für Soziologie und Sozialpsychologie, Jg. 34: S. 717-731.

Elwert, G. (1984), Die Angst vor dem Ghetto, S. 51-74, in: A. Bayas, M. Damolin und H. Ernst (Hrsg.), Integration: Anpassung an die Deutschen?, Weinheim, Basel.

Endruweit, G. (1975), Akkulturationstheorien in der Gastarbeiterforschung. Dritte Welt, Jg. 4, S. 226-258.

Engel, U. und Reinecke, J. (1994), Panelanalyse - Grundlagen, Techniken, Beispiele. Berlin.

Ernst, H. (2002), Die schleswig-holsteinische Amerika-Auswanderung und Rückwanderung in der Zeit der Weimarer Republik. Bochum.

Espinosa, K. und Massey, D. (1999), Undocumented Migration and the Quantity and Quality of Social Capital, S. 106-137, in: L. Pries, Migration and Transnational Social Spaces, Aldershot.

Esser, H. (1980), Aspekte der Wanderungssoziologie - Assimilation und Integration von Wanderern, ethnischen Gruppen und Minderheiten. Eine handlungstheoretische Analyse. Darmstadt, Neuwied. 
Esser, H. (1981), Aufenthaltsdauer und die Eingliederung von Wanderern: Zur theoretischen Interpretation soziologischer „Variablen“. Zeitschrift für Soziologie, Jg. 10: S. 76-97.

Esser, H. (1982), Sozialräumliche Bedingungen der sprachlichen Assimilation von Arbeitsimigranten. Zeitschrift für Soziologie, Jg. 11: S. 279-306.

Esser, H. (1985), Soziale Differenzierung als ungeplante Folge absichtsvollen Handelns: Der Fall der ethnischen Segmentation. Zeitschrift für Soziologie, Jg. 14: S. 435-449.

Esser, H. (1986), Ethnische Kolonien: Binnenintegration oder gesellschaftliche Isolation?, S. 106-117, in: J.P. Hoffmeyer-Zlotnik, (Hrsg.), Segregation und Integration - Die Situation von Arbeitsmigranten im Aufnahmeland, Mannheim.

Esser, H. (1988), Ethnische Differenzierung und moderne Gesellschaft. Zeitschrift für Soziologie, Jg. 17: S. 235-248.

Esser, H. (1991), The Integration of Second Generation Immigrants in Germany: An Explanation of „Cultural“ Differences, S. 45-69, in: R. S. Sigel und M. Hoskins (Hrsg.), Education of Democratic Citizenship: A Challange for Multi-Ethnic Societies, Hillsdale.

Esser, H. (1990), Nur eine Frage der Zeit? Zur Eingliederung von Migranten im Generationen-Zyklus und zu einer Möglichkeit, Unterschiede hierin zu erklären, S. 73-100, in: H. Esser und J. Friedrichs (Hrsg.), Generation und Identität. Theoretische und empirische Beiträge zur Migrationssoziologie. Opladen.

Esser, H. (1996), Die Definition der Situation. Kölner Zeitschrift für Soziologie und Sozialpsychologie, Jg. 48: S. 1-33.

Esser, H. (1997), Entstehung ethnischer Konflikte, S. 876-894, in: S. Hradil (Hrsg.), Differenz und Integration: Die Zukunft der modernen Gesellschaft, Opladen.

Esser, H. (1999a), Die Situationslogik ethnischer Konflikte. Zeitschrift für Soziologie, Jg. 28: S. 245-262.

Esser, H. (1999b), Soziologie - Allgemeine Grundlagen, Frankfurt/Main.

Esser, H. (2001), Integration und ethnische Schichtung. MZES Arbeitspapiere, Nr. 40, Mannheim. 
Esser, H. (2003a), Does the New Immigration Require a New Theory of Intergenerational Integration? MZES Arbeitspapiere, Nr. 71, Mannheim.

Esser, H. (2003b), Ist das Konzept der Assimilation überholt? Geographische Revue, Jg. 5: S. 5-39.

Esser, H. (2006), Sprache und Integration - Die sozialen Bedingungen und Folgen des Spracherwerbs von Migranten. Frankfurt/Main.

Esser, H. und Friedrichs, J. (Hrsg.) (1990), Generation und Identität - Theoretische und empirische Beiträge zur Migrationssoziologie, Opladen.

Evans, M.D.R. (1988), Choosing to be a Citizen: the time path if citizenship. International Migration Review, Jg. 27: S. 115-144.

Fainstain, N. (1987), The Underclass/Mismatch Hypothesis as an Explanation for Black Economic Deprivation. Politics and Society, Jg. 15: S. 403-451.

Faist, T. (1995), Social Citizenship for Whom? Young Turks in Germany and Mexican Americans in the United States, Aldershot.

Faist, T. (1999), Developing Transnational Social Spaces: The Turkish-German Example, S. 36-72, in: L. Pries, Migration and Transnational Social Spaces, Aldersshot.

Faist, T. (2000), The Volume and Dynamics of International Migration and Transnational Social Spaces.

Fertig, M. (2004), The Societal Integration of Immigrants in Germany. IZA: Discussion Paper, Nr. 1213.

Frick, J. R. (2004), Gutachten zur „Integration von Migranten in Deutschland“ auf Basis nationaler und internationaler vergleichbarer repräsentativer Mikrodaten, im Auftrag des Sachverständigenrats für Zuwanderung und Immigration, Berlin.

Ganter, S. (2003), Soziale Netzwerke und interethnische Distanz - Theoretische und empirische Analysen zum Verhältnis von Deutschen und Ausländern, Wiesbaden.

Ganzeboom, H. B. G., De Graaf, P. M., Treiman, D. J. und de Leeuw, J. (1992), A Standard International Socio-Economic Index of Occupational Status. Social Science Research, Jg. 21: S. 1-56. 
Geis, A. J. und Hoffmeyer-Zlotnik, J. H. P. (2001), Kompatibilität von ISCO-68, ISCO-88 und KLDB-92. ZUMA-Nachrichten, Nr. 48: S.117-138.

Geißler, R. (2003), Multikulturalismus in Kanada - Modell für Deutschland? Aus Politik und Zeitgeschichte, Heft 26: S. 19-25.

Gilroy, P. (1987): There Ain't No Black in Union Jack, Hutchinson, London.

Glasgow, Douglas G. (1980): The Black Underclass. Poverty, Unemployment and Entrapment of Ghetto Youth. San Francisco, Washington, London.

Glazer, N. (1957), American Judaism, Chicago.

Glazer, N. und Moynihan, D. P. (1963), Beyond the Melting Pot. The Negroes, Puerto Ricans, Jews, Italians and Irish of New York City. Cambridge.

Glick-Schiller, N., Basch, L. und Blanc-Szanton, C. (1999), From Immigrant to Transmigrant. Theorizing Transnational Migration, S. 73-105 in: L. Pries (Hrsg.), Migration and Transnational Social Spaces, Aldershot.

Gordon, M. M. (1964), Assimilation in American Life. The Role of Race, Religion and National Origins. New York.

Haines, D. W. und Rosenblum, K. E. (Hrsg.) (1999), Illegal Immigration in America. A Reference Handbook. Westport.

Haisken-DeNew, J. P. und Frick, J. R. (Hrsg.) (2003), Desktop Companion to the German Socio-Economic Panel Study (SOEP), Berlin.

Halfmann, J. und Bommes, M. (1998), Staatsbürgerschaft, Inklusionsvermittlung und Migration. Zum Souveränitätsverlust des Wohlfahrtsstaates, S. 81-101, in: M. Bommes, und J. Halfmann (Hrsg.), Migration in nationalen Wohlfahrtsstaaten. Theoretische und vergleichende Untersuchungen. IMIS-Schriften, Bd. 6, Osnabrück.

Hall, S. (1980), Articulation and Societies Structured in Dominance in UNESCO, S. 305-345, in: UNESCO (Hrsg.), Sociological theories, race and colonialism, Paris.

Han, P. (2000) Soziologie der Migration, Stuttgart.

Haug, S. (1998), Anomalien in der Entscheidungstheorie. Empirische Evidenz und Konsequenzen, S. 126-160, in: U. Druwe und V. Kunz (Hrsg.), Anomalien in der Handlungs- und Entscheidungstheorie, Opladen. 
Haug, S. (2000a), Soziales Kapital und Kettenmigration - Italienische Migranten in Deutschland, Opladen.

Haug, S. (2000b), Klassische und neuere Theorien der Migration, MZES Arbeitspapiere Nr. 30, Mannheim.

Haug, S. (2001), Bleiben oder Zurückkehren? Zur Messung, Erklärung und Prognose der Rückkehr von Immigranten in Deutschland. Zeitschrift für Bevölkerungswissenschaft, Jg. 26: S. 231-270.

Haug, S. (2005), Die Datenlage im Bereich der Migrations- und Integrationsforschung. Ein Überblick über wesentliche Migrations- und Integrationsindikatoren und die Datenquellen. Bundesamt für Migration und Flüchtlinge, Working Papers, Nr. 1, Nürnberg.

Hayduk, L. A. (1996), LISREL Issues, debates, and strategies. Baltimore, London.

Heckmann, F. (1992), Ethnische Minderheiten, Volk und Nation. Soziologie interethnischer Beziehungen, Stuttgart.

Heitmeyer, W., Müller, J. und Schröder, H. (1997), Verlockender Fundamentalismus. Türkische Jugendliche in Deutschland, Frankfurt.

Hempel, C. G. und Oppenheim, P. (1948), Studies in the Logic of Explanation. Philosophy of Science, Jg.15: S. 135-175.

Herberg, W. (1960), Protestant - Catholic - Jew, New York.

Herbert, U. (2001), Geschichte der Ausländerpolitik in Deutschland, München.

Hill, P. B. (1984), Determinanten der Eingliederung von Arbeitsmigranten, Königstein.

Hoerder, D. (1985), Acculturation Twice: Return Migration, S. 353-355, in: D. Hoerder (Hrsg.), Labor Migration in the Atlantic Economies - The European and North American Working Classes During the Period of Industrialization, London.

Hoerder, D. (2002), Europäische Migrationsgeschichte und Weltgeschichte der Migration: Epochenzäsuren und Methodenprobleme. IMIS-Beiträge, Heft 20: S. 135-167. 
Hoffmeyer-Zlotnik, J. H. P. (1993), Operationalisierung von „Beruf“ als zentrale Variable zur Messung von sozio-ökonomischem Status. ZUMA-Nachrichten, Nr. 32: S. $135-141$.

Hoffmeyer-Zlotnik, J. H. P. und Warner, U. (1998), Die Messung von Einkommen im nationalen und internationalen Vergleich. ZUMA-Nachrichten Nr. 42: S. 30-65.

Hunger, U. und Thränhardt, D. (2004), Migration und Bildungserfolg: Wo stehen wir? IMIS-Beiträge, Heft 23: S. 179-198.

Institut der deutschen Wirtschaft (2001), Deutschland in Zahlen, Köln.

Institut der deutschen Wirtschaft (2005), Deutschland in Zahlen, Köln.

Jacobson, D. (1996), Rights across Borders. Immigration and the Decline of Citizenship, Baltimore.

Jankowitsch, B, Klein, T. und Weick, S. (2000), Die Rückkehr ausländischer Arbeitsmigranten seit Mitte der achtziger Jahre, S. 93-109, in: R. Alba, P. Schmidt und M. Wasmer, Deutsche und Ausländer: Freunde, Fremde oder Feinde?, Wiesbaden.

Jasso, G. und Rosenzweig, M. R. (1986), Family Reunification and the Immigration Multiplier: U.S. Immigration Law, Origincountry Conditions and the Reproduction of Immigrants. International Migration Review, Jg. 23: S. 291-311.

Jasso, G und Rosenzweig, M. R. (1990), English language proficiency and the locational choices of immigrants, S. 308-337, in: G. Jasso und M. R. Rosenzweig, The new chosen people: Immigrants in the United States, New York.

Jöreskog, K. G. (1993), Testing structural equation models, S. 294-316, in: K. A. Bollen und S. J. Long (Hrsg.), Testing structural equation models, Newburry Park.

Kalter, F. und Granato, N. (2004), Sozialer Wandel und strukturelle Assimilation in der Bundesrepublik. Empirische Befunde mit Mikrodaten der amtlichen Statistik. IMIS-Beiträge, Heft 23: S. 61-81.

Koch-Kraft, A. (1990), Deutsche in Kanada - Einwanderung und Adaptation, Bochum. 
Kolb, H. (2004), Covert Doors: German Immigration Policy between Pragmatic Policy-Making and Symbolic Representation. IMIS-Beiträge, Heft 24: S. 129-136.

Korte, E. (1990), Die Rückkehrorientierung im Eingliederungsprozeß von Migrantenfamilien, S. 207-259, in: H. Esser und J. Friedrichs (Hrsg), Generation und Identität. Theoretische und empirische Beiträge zur Migrationssoziologie, Opladen.

Kortum, G. (1981), Migrationstheoretische und bevölkerungsgeographische Probleme der nordfriesischen Amerikarückwanderung, S. 111-201, in: K. D. Sievers (Hrsg.), Die deutsche und skandinavische Amerikaauswanderung im 19. und 20. Jahrhundert, Neumünster.

Krassowski, W. (1963), Naturalization and Assimilation-proneness of California Immigrant Populations: A Statistical Study. Unveröffentlichte Dissertation. University of California, Los Angeles.

Kristen, C. (2000), Ethnic Differences in Educational Placement: The Transition from Primery to Secondary Schooling, MZES Arbeitspapiere Nr. 32, Mannheim.

Kristen, C. (2002), Hauptschule, Realschule oder Gymnasium? Ethnische Unterschiede am ersten Bildungsübergang. Kölner Zeitschrift für Soziologie und Sozialpsychologie, Jg. 54: S. 534-552.

Kronauer, M. (2002), Exklusion - Die Gefährdung des Sozialen im hoch entwickelten Kapitalismus, Frankfurt/Main.

Krumme, H. (2004), Fortwährende Remigration: Das transnationale Pendeln türkischer Arbeitsmigrantinnen und Arbeitsmigranten im Ruhestand. Zeitschrift für Soziologie, Jg. 33: S. 138-153.

Kühnel, S. (1993), Lassen sich ordinale Daten mit linearen Strukturgleichungsmodellen analysieren? ZA-Information, Nr. 33: S. 29-51.

Kühnel, S. und Leibold, J. (2000), Die anderen und wir: Das Verhältnis zwischen Deutschen und Ausländern aus der Sicht der in Deutschland lebenden Ausländer, S. 111-146, in: R. Alba, P. Schmidt und M. Wasmer (Hrsg.), Deutsche und Ausländer: Freunde, Fremde oder Feinde?, Wiesbaden.

Leggewie, C. (1990), Multi Kulti, Spielregeln für die Vielvölkerrepublik, Berlin. 
Leibold, J. (2004), Die Entwicklung der Integrationsbereitschaft von Immigranten und Deutschen, S. 293-309, in: P. Wolf, und S. Rück (Hrsg.), Wir und das Fremde - Nell-Breuning Symposium Rödermark Oktober 2002, Münster.

Leibold, J. und Kühnel, S. (2006): Islamophobie. Differenzierung tut Not, S. 135155, in: W. Heitmeyer (Hrsg.), Deutsche Zustände, Bd. 4, Frankfurt/M.

Liang, Z. (1994), On the Measurement of Naturalization. Demography, Jg. 31: S. 525-548.

Lindenberg, S. (1985), An Assessment of the New Political Economy. Its Potential for the Social Sciences and for Sociology in Particular. Sociological Theory Jg. 3: S. 99-114.

Lindenberg, S. (1989), Social Production Functions, Deficits, and Social Revolutions. Prerevolutionary France and Russia. Rationality and Society, Jg. 1: S. 5177.

Lindenberg, S. (1993), Framing, Empirical Evidence, and Applications, S. 11-49, in: P. Herder-Dorneich, K.-E. Schenk und D. Schmidtchen (Hrsg.), Jahrbuch für Neue Politische Ökonomie, Tübingen.

Löffelholz v., H. D., Bauer, T.,Haisken-DeNew, J. und Schmidt, C. M. (2004), Fiskalische Kosten der Zuwanderer. RWI, Endbericht zum Forschungsvorhaben des Sachverständigenrates für Zuwanderung und Integration, Essen.

Lopez, D. E. (1999), Social and linguistic aspects of assimilation today, S. 212-222, in: C. Hirschman, P. Kasinitz und J. DeWind (Hrsg.), The handbook of international migration: the American experience, New York.

Loury, G. C. (2000), Social Exclusion and Ethnic Groups: The Challenge to Economics, S. 225-254, in: B. Pleskovic und J. E. Stiglitz (Hrsg.), The World Bank, Washington.

Lucassen, L. (2004), Assimilation in Westeuropa seit der Mitte des 19. Jahrhunderts: historische und historiographische Erfahrungen, S. 43-66, in : K. J. Bade, M. Bommes und R. Münz (Hrsg.), Migrationsreport 2004: Fakten, Analyen, Perspektiven, Frankfurt/Main, New York.

Marshall, Thomas H. (1950): Citizenship and Social Class, Cambridge. 
Martiniello, M. (1994), Citizenship of the European Union. A Critical View, S. 29-47 in: R. Bauböck (Hrsg.), From Aliens to Citizens. Redefining the Status of Immigrants in Europe.

Massey, D. S. und Denton, N. A. (1993): American Apartheid. Segregation and the Making of the Underclass, London.

McNeil, W. H. und Adams, R. S. (1978), Human Migration: Patterns and Policies, London.

Mehrländer, U. (1987), Ausländerforschung 1965 bis 1980, Fragestellungen, theoretische Ansätze, empirische Ergebnisse, Bonn.

Mehrländer, U., Ascheberg, C. und Ueltzhöffer, J. (1996), Repräsentativuntersuchung '95: Situation der ausländischen Arbeitnehmer und ihrer Familienangehörigen in der Bundesrepublik Deutschland, Berlin.

Meis, N. (1993), Aspekte struktureller und differentieller Mobilität von Ausländern in der Bundesrepublik Deutschland, Wiesbaden.

Mintzel, A. (1997), Multikulturelle Gesellschaften in Europa und Nordamerika - Konzepte, Streitfragen, Analysen, Befunde, Passau.

Möser, T. (2005), Remigration von „Gastarbeitern“ in ihre Herkunftsländer - Eine Verlaufsdatenanalyse mit dem Sozio-oekonomischen Panel, Diplomarbeit, GieBen.

Münz, R. und Ulrich, R. (2000), Die ethnische und demographische Struktur von Ausländern und Zuwanderern in Deutschland, S. 11-54, in: R. Alba, P. Schmidt und M. Wasmer (Hrsg.), Deutsche und Ausländer: Freunde, Fremde oder Feinde?, Wiesbaden.

Nassehi, A. (1995), Der Fremde als Vertrauter. Soziologische Betrachtungen zur Konstruktion von Identitäten und Differenzen. Kölner Zeitschrift für Soziologie und Sozialpsychologie, Jg. 47: S. 443-463.

Nauck, B. (1988), Zwanzig Jahre Migrantenfamilien in der Bundesrepublik. Familiärer Wandel zwischen Situationsanpassung, Akkulturation und Segregation, S. 279-297, in: R. Nave-Herz, Wandel und Kontinuität der Familie in der Bundesrepublik Deutschland, Stuttgart. 
Nekby, L. (2004), The Emigration of Immigrants, Return vs. Onward Migration: Evidence from Sweden, in: Stockholm University, Department of Economics, Research Papers in Economics, Nr. 7, Stockholm.

Opp, K. D. und Schmidt, P. (1995), Einführung in die Mehrvariablenanalyse, Reinbeck.

Opp, K. D. (1995), Methodologie der Sozialwissenschaften. Einführung in Probleme ihrer Theoriebildung und praktische Anwendungen, Opladen.

Özcan, V. (2002), Einbürgerungsverhalten von Ausländern - beeinflussende Faktoren auf der Angebots- und Nachfrageseite, Berlin.

Park, R. E. (1928), Human Migration and the Marginal Man. American Journal of Sociology, Jg. 33: S. 881-893.

Park, R. E. (1914), Racial Assimilation in Secondary Groups With Particular Reference to the Negro. American Journal of Sociology, Jg. 19: S. 606-623.

Paugam, Serge (1994): La disqualification sociale, Essai sur la nouvelle pauvreté. Paris.

Pischke, J. S. (1993), Assimilation and the Earnings of Guest workers in Germany, Discussion Paper, University of Mannheim.

Popper, K. R. (1965), Das Elend des Historizismus. Tübingen.

Portes, A. und Rumbaut, R. G. (2001), Lost in translation. Language and the new second generation, S. 113-146, in: A. Portes und R. G. Rumbaut, Legacies. The story of the Immigrant second generation, Berkeley.

Price, C.(1964), Jewish Settlers in Australia, Canberra.

Price, C. (1969), The Study of Assimilation, S. 181-237, in: J. A. Jackson, Migration, Cambridge.

Pries, L. (2001), New Transnational Social Spaces. International Migration and Transnational Companies, London.

Pries, L. (2002), Transnationalisierung der sozialen Welt? Berliner Journal für Soziologie, Jg. 12: S. 263-272. 
Pries, L. (2003), Transnationalismus, Migration und Inkorporation. Herausforderungen an Raum- und Sozialwissenschaften. Geographische Revue, Jg. 5: S. 23-39.

Radtke, F.-O. (1998), Lob der Gleich-Gültigkeit. Die Konstruktion des Fremden im Diskurs des Multikulturalismus, S. 79-96, in : U. Bielefeld (Hrsg.), Das Eigene und das Fremde - Neuer Rassismus in der Alten Welt?, Hamburg.

Radtke, Frank-Olaf (2004): Die Illusion der meritokratischen Schule. Lokale Konstellationen der Produktion von Ungleichheit im Erziehungssystem, in: K. J. Bade und M. Bommes, IMIS-Beiträge Themenheft: Migration - Integration - Bildung. Grundfragen und Problembereiche, Heft 23, S. 143-178, Osnabrück.

Ramsey, F. P. (1931), Truth and Probability, S. 156-198, in: R. B. Braithwaite (Hrsg.), The Foundations of Mathematics and other Logical Essays, London.

Reinecke, J. (2005), Strukturgleichungsmodelle in den Sozialwissenschaften, München.

Reagan, P. B. und Olsen, R. J. (2000), You Can Go Home Again: Evidence from Longitudinal Data. Demography, Jg. 37: S. 339-350.

Reckwitz, A. (2001), Multikulturalismustheorien und der Kulturbegriff: Vom Homogenitätsmodell zum Modell kultureller Interferenzen. Berliner Journal für Soziologie, Jg. 11: S. 179-200.

Rex, J. und Moore, R. (1967): Race, Community and Conflict, London.

Rheinisch-Westfälisches Institut für Wirtschaftsforschung e.V. (RWI) (2001), Berufliche Integration von Zuwanderern, ein Gutachten im Auftrag der Unabhängigen Kommission „Zuwanderung“ beim Bundesminister des Innern, Essen.

Richter, E. (2005), Die Einbürgerung des Islam. Aus Politik und Zeitgeschichte, Heft 20, S. 3-7.

Ring, H. (1998), Einwanderungspolitik im schwedischen Wohlfahrtsstaat, S. 239249, in: M. Bommes und J. Halfmann (Hrsg.), Migration in nationalen Wohlfahrtsstaaten. Theoretische und vergleichende Untersuchungen, IMIS-Schriften, Bd. 6 Osnabrück.

Rosner, J. (2001), Illegalisierte Arbeitsmigrantinnen, agisra Nr. 30/31: S.28-37. 
Rousseau, J.-J. (1977) [1762], Gesellschaftsvertrag. Stuttgart.

Ruhloff, J. (1989), Harmonisierung oder Widerstreit? Über die Bildungsaufgabe in der Kulturenvielfalt. Materialien zur Politischen Bildung, Heft 2: S. 8-15.

Sachverständigenrat für Zuwanderung und Integration (2004), Migration und Integration - Erfahrungen nutzen, Neues wagen, Jahresgutachten, Berlin.

Saltentin, K. und Wilkening, F. (2003), Ausländer, Eingebürgerte und das Problem einer realistischen Zuwanderer-Integrationsbilanz. Kölner Zeitschrift für Soziologie und Sozialpsychologie, Jg. 55: S. 278-298.

Saloojee, A. (2003), Social Inclusion, Anti-Racism and Democratic Citizenship. Working paper series Perspectives on social inclusion, Toronto.

Schäfer, H. (2004), Möglichkeiten der qualitativen und quantitativen Ermittlung von Zuwanderungsbedarf in Teilarbeitsmärkten in Deutschland - Grundlagen einer Indikatorik für eine arbeitsmarktbezogene Zuwanderung. Expertise für den Sachverständigenrat für Zuwanderung und Integration, Nürnberg.

Schräpler, J.-P. (2000), Was kann man am Beispiel des SOEP bezüglich Nonresponse lernen? ZUMA-Nachrichten, Nr. 46: S.117-150.

Schultz, T. und Sackmann, R. (2001), „Wir Türken...“, Zur kollektiven Identität türkischer Migranten in Deutschland. Aus Politik und Zeitgeschichte, Heft 43: S. 4045 .

Schumacker, R. E. und Lomax R. G. (1996), A beginner's guide to structural equation modelling, Mahwah.

Schumpeter, J.A. (1908), Das Wesen und der Hauptinhalt der theoretischen Nationalökonomie. Leipzig.

Schütz, A. (1972) [1944], Der Fremde. Ein sozialpsychologischer Versuch, S. 5369, in: ders., Gesammelte Aufsätze, Bd. 2, Studien zur soziologischen Theorie, Den Haag.

Seibel, H. D. und Nygreen, G. T. (1972), Pfadanalyse, ein statistisches Verfahren zur Untersuchung linearer Kausalmodelle. Zeitschrift für Sozialpsychologie, Sonderdruck, Bd. 3: S. 5-12. 
Șen, F., Sauer, M und Halm, D. (2001), Intergeneratives Verhalten und (Selbst-) Ethnisierung von türkischen Zuwanderern. Gutachten des ZfT für die Unabhängige Kommission „Zuwanderung“. Essen.

Sievers, K. D. (1981), Stand und Aufgaben der Überseewanderungsforschung in Schleswig-Holstein, S. 89-110, in: ders. (Hrsg.), Die deutsche und skandinavische Amerikaauswanderung im 19. und 20. Jahrhundert, Neumünster.

Simmel, Georg (1992) [1908], Soziologie: Untersuchungen über die Formen der Vergesellschaftung, Gesamtausgabe Bd. 11, Frankfurt/Main.

Statistisches Bundesamt (1997), Strukturdaten über die ausländische Bevölkerung, Wiesbaden.

Statistisches Bundesamt (2000): Bevölkerungsentwicklung Deutschlands bis 2050, Ergebnisse der 9. koordinierten Bevölkerungsvorausberechnung, Wiesbaden.

Statistisches Bundesamt (2005): Einbürgerungen im Jahr 2004, Pressemitteilung vom 20. Juli 2005, Wiesbaden.

Steiner, V. und Velling, J. (1992), Re-Migration Behaviour and Expected Duration of Stay of Guest-Workers of Germany, ZEW Discussion Paper, Nr. 92-14, Mannheim.

Stobbe, H. (2004): Undokumentierte Migration in Deutschland und den Vereinigten Staaten. Interne Migrationskontrollen und die Handlungsspielräume von Sans Papiers. Göttingen.

Straßburger, G. (2003), Heiratsverhalten und Partnerwahl im Einwanderungskontext. Eheschließungen der zweiten Migrantengeneration türkischer Herkunft, Würzburg.

Taft, R. (1957), A Psychological Model for the Study of Social Assimilation. Human Relations, Jg. 10: S. 141-156.

Taft, R. (1973), Problems of Adjustment and Assimilation, S. 224-239, in: P. Watson (Hrsg.), Immigrants, Psychology and Race, Harmondsworth.

Taylor, C. (1992), Die Politik der Anerkennung, S. 13-78, in: ders., Multikulturalismus und die Politik der Anerkennung, Frankfurt/Main. 
Tedebrand, L.-G. (1985), Remigration from America to Sweden, S. 357-380, in: D. Hoerder (Hrsg.), Labor Migration in the Atlantic Economies. The European and North American Working Classes During the Period of Industrialization, London.

Thomas, W. I./Znanieki, F. (1958) [1918/1920], The Polish peasant in Europe and America. New York.

Tränhardt, D. (1988), Die Bundesrepublik Deutschland - ein unerklärtes Einwanderungsland? Aus Politik und Zeitgeschichte, Heft 24, S. 3-13.

Treibel, A. (2003), Migration in modernen Gesellschaften - Soziale Folgen von Einwanderung, Gastarbeit und Flucht, Weinheim, München.

Treiman, D. J. (1977), Occupational Prestige in Comparative Perspective, New York.

Velling, J. (1994), The determinants of family reunification among German guestworkers, S. 126-132, in: R. V. Burkhauser und G. G. Wagner (Hrsg.), Proceedings of the 1993 international conference of German Socio-Economic Panel Study users, Berlin.

Vertovec, S. (1999), Conceiving and researching transnationalism, Ethnic and Racial Studies, Jg. 22: S. 447-462.

Virtanen, K. (1985), Finnish Migrants (1860-1930) in the Overseas Return Migration Movement, S. 381-398, in: D. Hoerder (Hrsg.), Labor Migration in the Atlantic Economies. The European and North American Working Classes During the Period of Industrialization, London.

Wagner, U./Dick van, R./Endrikat, K. (2002), Interkulturelle Kontakte. Die Ergebnisse lassen hoffen, S. 96-109, in: W. Heitmeyer (Hrsg), Deutsche Zustände, Bd. 1, Frankfurt/M

Wahlbeck, Ö. (1998), Transnationalism and Diasporas: The Kurdish Example, Paper presented at the International Sociological Association XIV World Congress of Sociology, Montreal.

Warner, W. L. und Srole, L. (1945), The Social Systems of American Ethnic Groups, Yankee City Series, Bd 3, New Haven.

Weber, M. (1980) [1922], Wirtschaft und Gesellschaft. Grundriß der verstehenden Soziologie, Tübingen. 
Werth, M. (1983), Rückkehr- und Verbleibeabsichten türkischer Arbeitnehmer. Mitteilungen aus der Arbeitsmarkt- und Berufsforschung, Jg. 16: S. 345-359.

Wilson, W. J. (1980): The Declining Significance of Race. Blacks and Changing American Institutions. Chicago, London.

Winkler, B. (Hrsg.) (1993), Zukunftsangst Einwanderung, München.

Wippler R. (1987), Kulturelle Ressourcen, gesellschaftlicher Erfolg und Lebensqualität, S. 221-253, in: B. Giesen und H. Haferkamp (Hrsg.), Soziologie der sozialen Ungleichheit, Opladen.

Wirth, L. (1928), The Ghetto, Chicago.

Wolf, C. (1995), Sozio-Ökonomischer Status und Berufliches Prestige. ZUMANachrichten, Nr. 37, S. 102-136.

Yang, P. Q. (1994), Explaining Immigrant Naturalization. International Migration Review, Jg. 28: S. 449-477.

Yang, P. Q. (2002), Citizenship Acquisition of Post-1965 Asian Immigrants. Population and Environment, Jg. 23: S. 377-404.

Zentrum für Türkeistudien (2000), Die Lebenssituation und Partizipation türkischer Migranten in Nordrhein-Westfalen, Ergebnisse der zweiten Mehrthemenbefragung, Essen.

Zentrum für Türkeistudien (2005), Religiöse Praxis und organisatorische Vertretung türkischstämmiger Muslime in Deutschland, Ergebnisse einer telefonischen Befragung, Essen. 
Anhang

Personenfragebogen

des

Sozio-oekonomischen Panels

der Welle 1999 
Infratest Burke Sozialforschung

Landsberger Str. 338

80687 München

Tel.: $\quad 089 / 56000$

\section{Leben in Deutschland}

Befragung '99

zur sozialen Lage

der Haushalte

\section{Personenfragebogen für alle}

Dieser Fragebogen richtet sich an die einzelnen Personen im Haushalt.

Ihre Mitarbeit ist freiwillig. Die wissenschaftliche Aussagekraft dieser Untersuchung hängt aber entscheidend von der Mitarbeit aller Personen in allen Haushalten ab.

Darum bitten wir Sie herzlich,

- unserem Mitarbeiter dieses Interview zu gewähren

- oder den Fragebogen sorgfältig selbst auszufüllen.

Bitte vor Übergabe unbedingt eintragen

It. Adressenprotokoll:

Nr. des Haushalts:

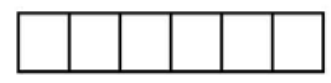

Person Nr::

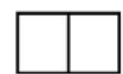

Vorname:

Bitte in Druckbuchstaben 
Wie wird's gemacht?

Bitte füllen Sie den Fragebogen aus, indem Sie

- in die weißen Kästchen ein Kreuz machen

Beispiel:

Geschlecht:

männlich

凶

weiblich

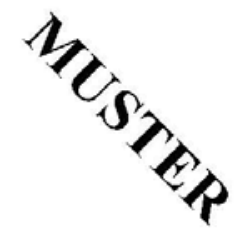

- in die etwas größeren weißen Felder Zahlen eintragen, und zwar rechtsbündig

Beispiel 1:

Miete

\begin{tabular}{|l|l|l|l|}
\hline & 9 & 5 & 0 \\
\hline
\end{tabular}

Beispiel 2:

Monat

$1 \quad 2$

(= Dezember)

- in die Balken Text schreiben

Beispiel:

Sonstiger Grund

区

und inar: Berufswechsel

Das Zeichen steht vor Erläuterungen zu einzelnen Fragen.

Gehen Sie bitte der Reihe nach vor, Frage für Frage. Überspringen Sie Fragen nur dann, wenn im Text ausdrücklich ein entsprechender Hinweis gegeben ist.

Beispiel:

Gibt es In Ihrem Haushalt Kinder?

$\mathrm{Ja}$

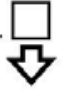

Nein

Sie springen auf Frage ...!

Wenn Sie in diesem Beispiel "Ja“

Wenn Sie in diesem Beispiel "Nein" ankreuzen, ankreuzen, geht es für Sie weiter geht es für Sie weiter mit der Frage, die im Hinweis mit der nächsten Frage!

"Sie springen auf Frage . . . !" angegeben ist!

\section{Und noch eine Bitte:}

Der Fragebogen wird mit der modernen Scanner-Technik ausgewertet. Im Prinzip hat jeder von Ihnen so einen Scanner schon in Betrieb gesehen: An der Kasse eines jeden größeren Geschäftes fährt die Kassiererin mit einem Scanner über das Preisschild der gekauften Ware, und schon ist die Information automatisch "gelesen". So ähnlich machen wir es mit dem Fragebogen. Damit das funktioniert, ist es sehr wichtig,

- daß Sie nur einen schwarzen oder blauen Kugelschreiber verwenden

- daß Ihre Angaben gut lesbar sind

- und daß Ihre Markierung innerhalb der Kästchen bleibt.

Sie erleichtern uns dadurch sehr die Arbeit. Vielen Dank! 


\section{Ihre Lebenssituation heute}

1. Wie zufrieden sind Sie heute mit den folgenden Bereichen Ihres Lebens?

Bitte kreuzen Sie fur jeden Bereich auf der Skala einen Wert an:

Wenn Sie ganz und gar unzufrieden sind, den Wert " $O$ ",

wenn Sie ganz und gar zufrieden sind, den Wert "10"

Wenn Sie teils zufrieden / teils unzufrieden sind, einen Wert dazwischen

Wie zufrieden sind Sie...

- mit Ihrer Gesundheit?

(falls Sie enwerbstatig sind)

- mit Ihrer Arbeit?

(falls Sie im Haushalt tätig sind)

- mit Ihrer Tätigkeit im Haushalt?

- mit dem Einkommen

Ihres Haushalts?

- mit Ihrer Wohnung?

- mit Ihrer Freizeit?

(falls Sie kleine Kinder haben)

- mit den vorhandenen Möglichkeiten der Kinderbetreuung?

- mit dem Angebot an Waren und Dienstleistungen hier am Ort?

- mit der Wohngegend hier?

- mit dem Zustand der Umwelt hier in der Region?

- mit Ihrem Lebensstandard insgesamt? ganz und gar ganz und gar unzufrieden zufrieden
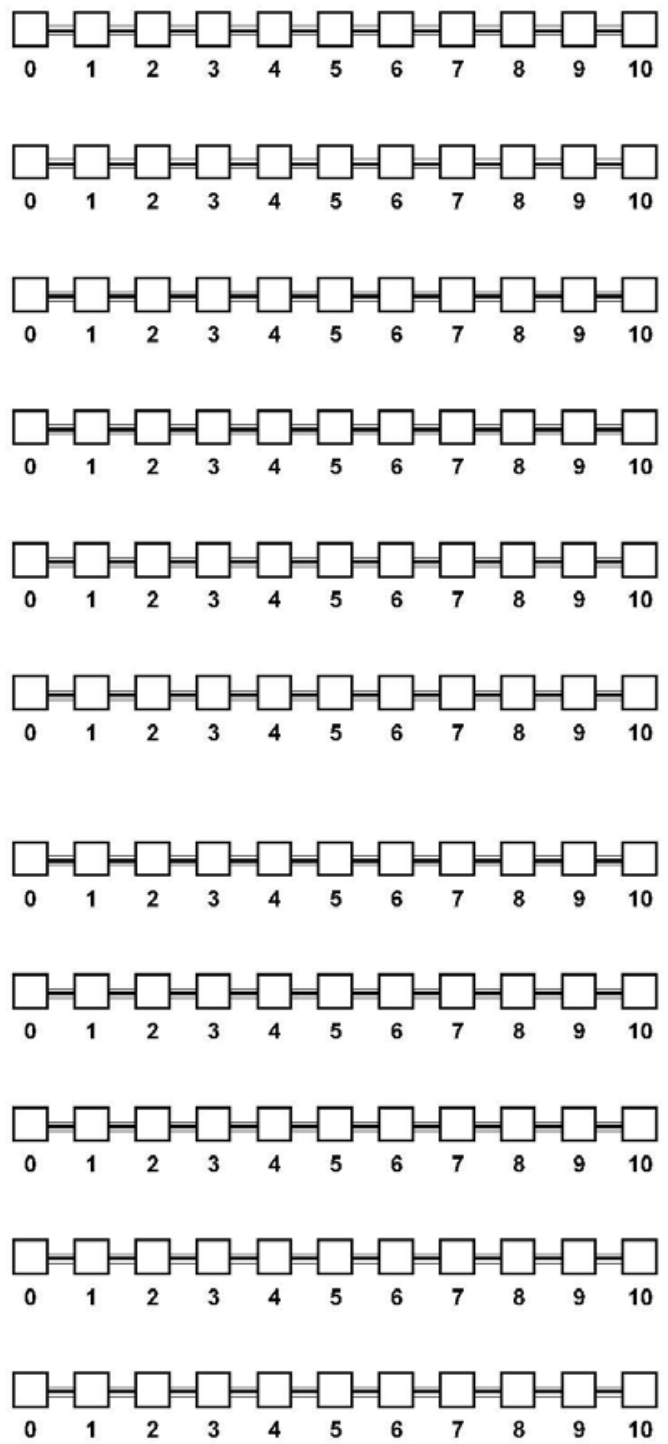


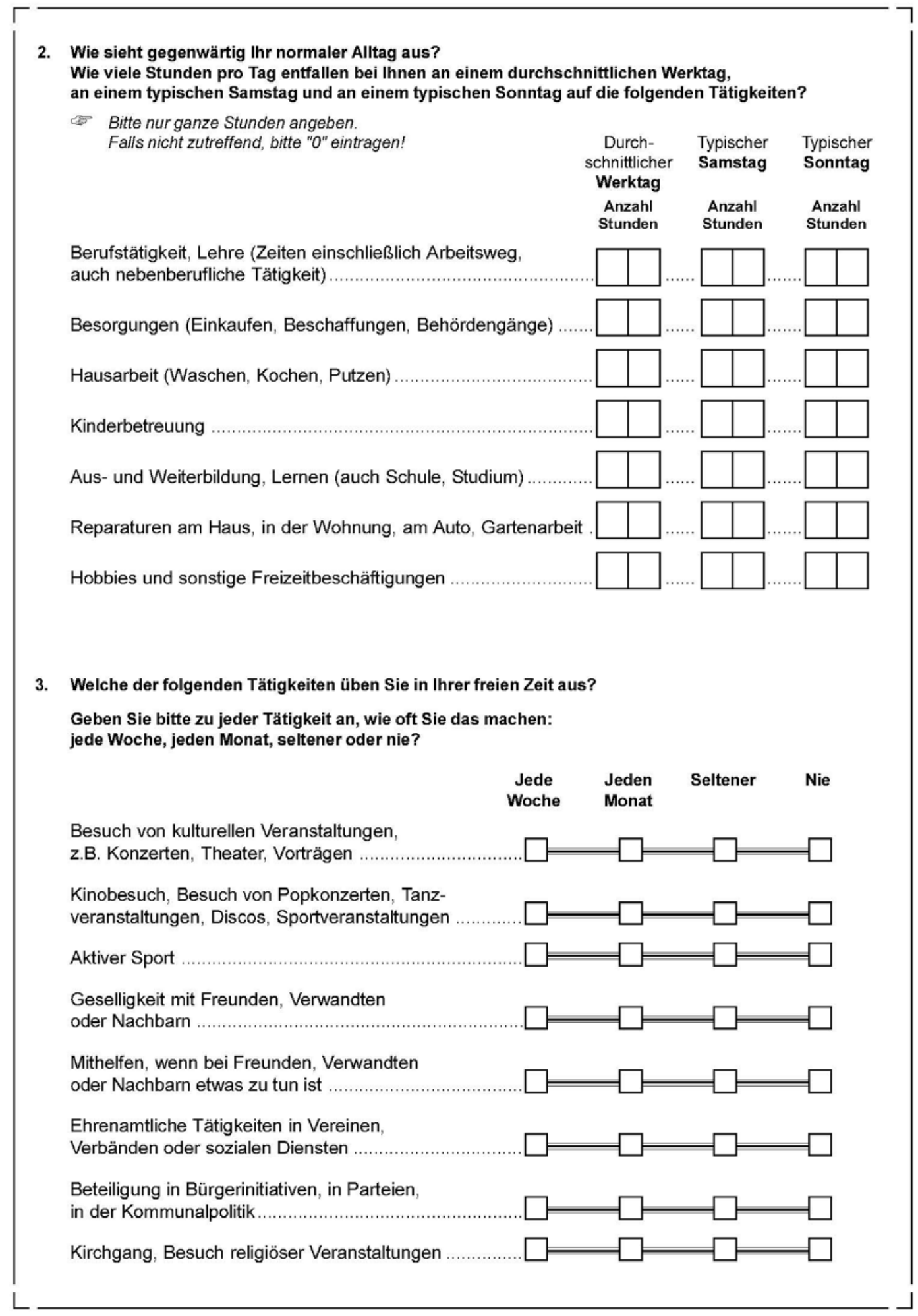




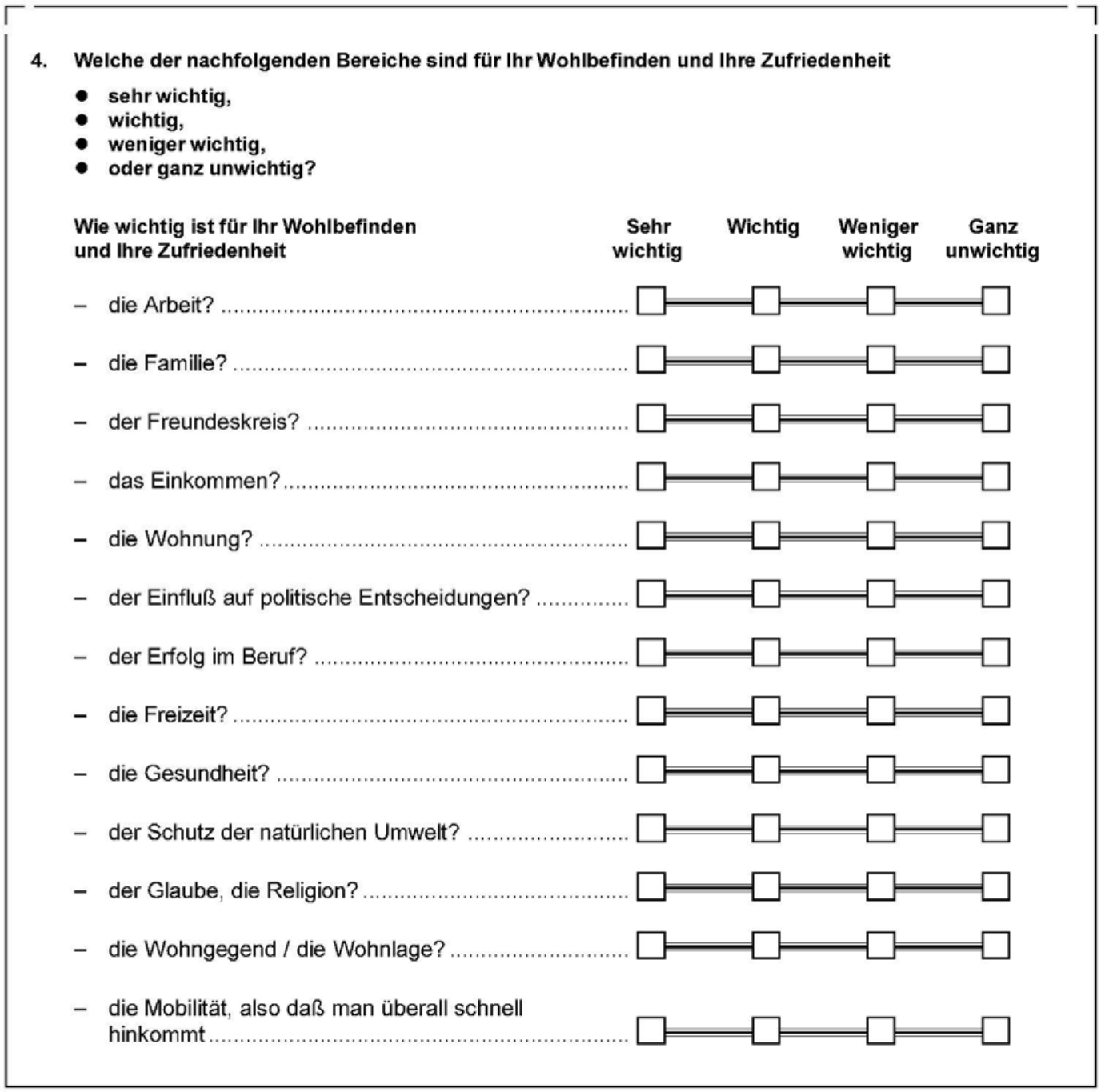

5. Sind Sie zur Zeit beim Arbeitsamt arbeitslos gemeldet?

$\mathrm{Ja}$

Nein

6. Sind Sie zur Zeit im Mutterschafts- oder Erziehungsurlaub?

$\mathrm{Ja}$

Nein

7. Haben Sie während der letzten 7 Tage irgendeine bezahlte Arbeit ausgeübt, auch wenn dies nur für eine Stunde oder für wenige Stunden war?

Bitte antworten Sie auch mit "Ja", wenn Sie in den letzten sieben Tagen normalerweise gearbeitet hätten, aber wegen Urlaub, Krankheit, schlechtem Wetter oder anderen Granden zeitweise abwesend waren.

$\mathrm{Ja}$

Nein 
8. Sind Sie derzeit in Ausbildung? Das heißt: Besuchen Sie eine Schule oder Hochschule, machen Sie eine Berufsausbildung oder nehmen Sie an einem Weiterbildungslehrgang teil?

Ja .................

Nein

Sie springen auf Frage 10 !

9. Was für eine Ausbildung oder Weiterbildung ist das?

Allgemeinbildende Schule

Hauptschule

Realschule

Gymnasium

Gesamtschule

Abendrealschule, Abendgymnasium

Fachoberschule

Hochschule

Fachhochschule

Universität, sonstige Hochschule

Lehrgang / Kursus zur Weiterbildung

Berufliche Umschulung

Berufliche Fortbildung .....

Berufliche Rehabilitation

Allgemeine oder politische Weiterbildung

Sonstiges

und

zwar:
Berufliche Ausbildung

Berufsgrundbildungsjahr

Berufsvorbereitungsjahr

Berufsschule ohne Lehre

Lehre

Berufsfachschule, Handelsschule

Schule des Gesundheitswesens

Fachschule

(z.B. Meister-, Technikerschule)

Beamtenausbildung

Sonstiges

und

zwar:

10. Üben Sie derzeit eine Erwerbstätigkeit aus?

Was trifft für Sie zu?

Rentner mit Arbeitsvertrag gelten hier als enwerbstätig.

Voll erwerbstätig

In Teilzeitbeschäftigung

In betrieblicher Ausbildung / Lehre

oder betrieblicher Umschulung

Geringfügig oder unregelmäßig erwerbstätig

Sie springen auf Frage 21!

Als Wehrpflichtiger beim Wehrdienst / Zivildienst

Bitte weiter mit Frage 11!

Nicht erwerbstätig 


\section{Nur an Nicht-Erwerbstätige:}

11. Wenn Sie jetzt eine Arbeit suchen würden:

Ist oder wäre es für Sie leicht, schwierig oder praktisch unmöglich, eine geeignete Stelle zu finden?

Leicht

Schwierig

Praktisch unmöglich

Trifft nicht zu, da Rentner / Pensionär

12. Beabsichtigen Sie, in der Zukunft (wieder) eine Erwerbstätigkeit aufzunehmen?

Nein, ganz sicher nicht

Sie springen auf Frage 61!

Eher unwahrscheinlich

Wahrscheinlich

Ganz sicher

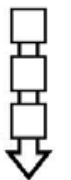

13. Wann etwa wollen Sie Ihre Erwerbstätigkeit aufnehmen?

Möglichst sofort

Innerhalb des kommenden Jahres

In 2 bis 5 Jahren

Erst später, in mehr als 5 Jahren

Weiß noch nicht

15. Möchten Sie in Ihrem bisherigen bzw. Ihrem erlernten Beruf arbeiten oder wollen Sie lieber etwas Neues machen, oder wäre Ihnen das egal?

Im bisherigen/erlernten Beruf

Etwas Neues machen

Trifft nicht zu, habe (noch)

Egal

keinen Beruf erlernt 
16. Wie wahrscheinlich ist es, daß innerhalb der nächsten 2 Jahre die folgenden beruflichen Veränderungen für Sie eintreten?

Bitte geben Sie die Wahrscheinlichkeit auf einer Skala von 0 bis 100 an

Der Wert 0 bedeutet: Das wird mit Sicherheit nicht eintreten.

Der Wert 100 bedeutet: Das wird mit Sicherheit eintreten.

Mit den Werten zwischen 0 und 100 können Sie die Wahrscheinlichkeit abstufen.

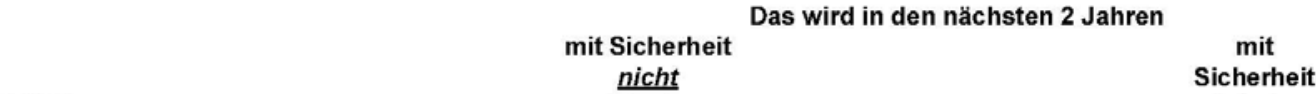

Daß Sie...

- eine bezahlte Beschäftigung aufnehmen? eintreten

- sich selbständig machen bzw. freiberuflich

tätig werden?
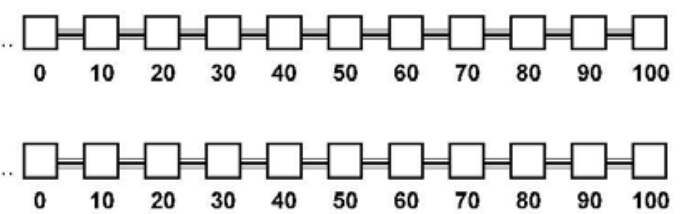

- sich durch Lehrgänge / Kurse weiterqualifizieren oder fortbilden?

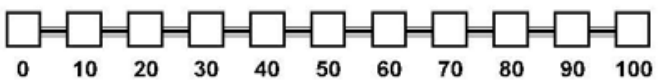

17. Es gibt ja verschiedene Gründe dafür, berufstätig zu sein.

Was steht bei Ihnen im Vordergrund: daß Sie Geld verdienen oder andere Gründe?

Geld verdienen

Andere Gründe

Beides gleichermaßen

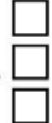

18. Wie hoch müßte der Nettoverdienst mindestens sein, damit Sie eine angebotene Stelle annehmen würden?

19. Wenn man Ihnen jetzt eine passende Stelle anbieten würde, könnten Sie dann innerhalb der nächsten zwei Wochen anfangen zu arbeiten?

$\mathrm{Ja}$

Nein ..........

20. Haben Sie innerhalb der letzten vier Wochen aktiv nach einer Stelle gesucht?

Ja

Nein ......

Sie springen auf Frage 61! 


\section{Ihre derzeitige Erwerbstätigkeit}

21. Haben Sie seit Anfang des vorigen Jahres, also seit Januar 1998, die Stelle gewechselt oder eine Arbeit neu aufgenommen?

Ja

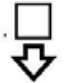

Nein

Sie springen auf Frage 36 !

22. Wann haben Sie Ihre jetzige Stelle angetreten bzw. die Arbeit aufgenommen?

1998, im Monat

1999, im Monat

23. Welche Art von beruflicher Veränderung war das?

Falls Sie mehrfach die Stelle gewechselt haben, sagen Sie es bitte for den letzten Wechsel.

Habe erstmals in meinem Leben eine

Erwerbstätigkeit aufgenommen

Habe nach einer Unterbrechung jetzt wieder eine

Erwerbstätigkeit aufgenommen

Habe eine Stelle bei einem neuen Arbeitgeber angetreten .

Habe eine neue Tätigkeit als Selbständiger aufgenommen

Bin vom Betrieb übernommen worden

(war vorher dort in Ausbildung / in ABM /

oder als freier Mitarbeiter tätig)

Habe innerhalb des Unternehmens die Stelle gewechselt

Frage 24 entfallt, bitte weiter mit Frage 25 
25. Wie beurteilen Sie Ihre heutige Stelle im Vergleich zur letzten?

In welchen Punkten haben Sie sich verbessert, welche sind etwa gleichwertig

und in welchen haben Sie sich eher verschlechtert?

In jede Zeile bitte eine Nennung!

Wie ist dies in bezug auf ...

Verbessert Etwa gleichwertig schlechtert

- die Art der Tätigkeit?

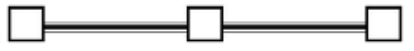

- den Verdienst?

- die Aufstiegsmöglichkeiten?

- die Arbeitsbelastungen?

- die Länge des Arbeitsweges?

- die Arbeitszeitregelung?

- die betrieblichen Sozialleistungen?

- und die Sicherheit des Arbeitsplatzes?

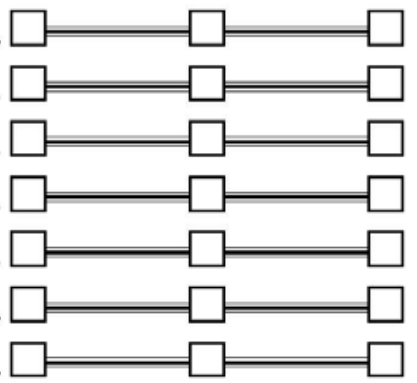

26. Können Sie von Ihren beruflichen Kenntnissen und Fähigkeiten heute mehr, gleich viel oder weniger verwenden als in Ihrer letzten Stelle?

Mehr ......

Gleich viel

Weniger

27. Bevor Sie ihre jetzige Stelle angetreten haben, haben Sie da aktiv nach einer Stelle gesucht oder hat sich das so ergeben?

Aktiv gesucht.

Hat sich so ergeben

28. Wie haben Sie von dieser Stelle erfahren?

Bitte nur einen Punkt ankreuzen!

Über das Arbeitsamt

Über eine private Stellenvermittlung / Agentur

Über eine Stellenanzeige in der Zeitung ....

Über eine Stellenanzeige im Internet.

Über Bekannte, Freunde, Angehörige

Bin zum früheren Arbeitgeber zurückgekehrt

Sonstiges bzw. nicht zutreffend 1 
29. Welche berufliche Tätigkeit üben Sie derzeit aus?

Bitte geben Sie die genaue Tatigkeitsbezeichnung an, also z. B. nicht "kaufmánnischer Angestellter", sondern: "Speditionskaufmann", nicht "Arbeiter", sondern: "Maschinenschlosser". Wenn Sie Beamter sind, geben Sie bitte Ihre Amtsbezeichnung an, z.B. "Polizeimeister", oder "Studienrat". Wenn Sie Auszubildender sind, geben Sie bitte Ihren Ausbildungsberuf an.

Bitte in Druckbuchstaben eintragen!

30. Entspricht diese Tätigkeit Ihrem erlernten Beruf?

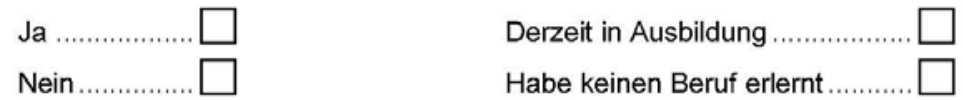

31. Welche Art von Ausbildung ist für diese Tätigkeit in der Regel erforderlich?

Keine bestimmte Ausbildung erforderlich

Eine abgeschlossene Berufsausbildung für diese Tätigkeit

Ein abgeschlossenes Fachhochschulstudium

Ein abgeschlossenes Universitäts- oder Hochschulstudium

32. Welche Art von Einarbeitung in diese Tätigkeit ist in der Regel erforderlich?

Nur eine kurze Einweisung am Arbeitsplatz

Eine längere Einarbeitung im Betrieb

Der Besuch von besonderen Lehrgängen / Kursen

33. Gehört der Betrieb, in dem Sie arbeiten, zum öffentlichen Dienst?

$\mathrm{Ja}$

Nein ........

34. In welchem Wirtschaftszweig / welcher Branche / welchem Dienstleistungsbereich ist das Unternehmen bzw. die Einrichtung überwiegend tätig?

Bitte geben Sie die genaue Bezeichnung an, z. B. nicht "Industrie", sondern "Elektroindustrie". nicht "Handel", sondern "Einzelhandel"; nicht "offentlicher Dienst", sondern "Krankenhaus".

Bitte in Druckbuchstaben eintragen!

35. Wieviele Beschäftigte hat das Gesamtunternehmen etwa?

\begin{tabular}{|c|c|}
\hline unter & 5 Beschäftigte \\
\hline 5 bis unter & 20 Beschäftigte \\
\hline 20 bis unter & 100 Beschäftigte \\
\hline 100 bis unter & 200 Beschäftigte \\
\hline 200 bis unter & .000 Beschäftig \\
\hline
\end{tabular}

Trifft nicht zu, da selbständig ohne weitere Beschäftigte 
36. Seit wann sind Sie bei Ihrem jetzigen Arbeitgeber beschäftigt?

Falls Sie Selbständiger sind, geben Sie bitte an seit wann Sie Ihre jetzige Tátigkeit ausäben.

Seit

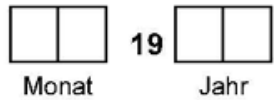

37. Haben Sie ein von vornherein befristetes Arbeitsverhältnis oder haben Sie einen unbefristeten Arbeitsvertrag?

Befristetes Arbeitsverhältnis $\square 5$ Handelt es sich um eine ABM-Stelle?

Habe unbefristeten Arbeitsvertrag Ja ........ $\square \quad$ Nein ...

Trifft nicht zu / bin Selbständiger

38. In welcher beruflichen Stellung sind Sie derzeit beschäftigt?

Wenn Sie mehr als eine berufliche Tätigkeit ausüben, beantworten Sie die folgenden Fragen bitte nur for Ihre derzeitige berufliche Haupttatigkeit.

\section{Arbeiter}

(auch in der Landwirtschaft)

Ungelernte Arbeiter

Angelernte Arbeiter

Gelernte und Facharbeiter

Vorarbeiter, Kolonnenführer

Meister, Polier

\section{Selbständige}

(einschl. mithelfende Familienangehörige)

Selbständige Landwirte

Freie Berufe,

selbständige Akademiker.

Sonstige Selbständige

- ohne Mitarbeiter

- mit 1 bis zu 9 Mitarbeitern

- mit 10 und mehr Mitarbeitern

Mithelfende Familienangehörige

\section{Auszubildende / Praktikanten}

Auszubildende

Volontäre, Praktikanten u.ä

\section{Angestellte}

Industrie- und Werkmeister

im Angestelltenverhältnis .

Angestellte mit einfacher Tätigkeit

- ohne Ausbildungsabschluß

- mit Ausbildungsabschluß

Angestellte mit qualifizierter Tätigkeit (z.B. Sachbearbeiter, Buchhalter, technischer Zeichner)

Angestellte mit hochqualifizierter Tätigkeit oder Leitungsfunktion (z.B. wissenschaftlicher Mitarbeiter, Ingenieur, Abteilungsleiter)

Angestellte mit umfassenden Führungsaufgaben (z.B. Direktor, Geschäftsführer, Vorstand größerer Betriebe und Verbände)

\section{Beamte}

(einschl. Richter und Berufssoldaten)

Einfacher Dienst

Mittlerer Dienst

Gehobener Dienst

Höherer Dienst 
39. Wie wahrscheinlich ist es, daß innerhalb der nächsten 2 Jahre die folgenden beruflichen Veränderungen für Sie eintreten?

Bitte geben Sie die Wahrscheinlichkeit auf einer Skala von 0 bis 100 an

Der Wert 0 bedeutet: Das wird mit Sicherheit nicht eintreten

Der Wert 100 bedeutet: Das wird mit Sicherheit eintreten.

Mit den Werten zwischen 0 und 100 können Sie die Wahrscheinlichkeit abstufen.

Daß Sie... $\quad \begin{gathered}\text { mit Sicherheit } \\ \text { nicht } \\ \text { eintreten }\end{gathered}$

- von sich aus eine neue Stelle suchen?

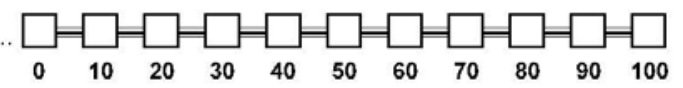

- Ihren Arbeitsplatz verlieren?

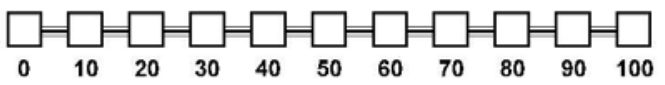

- in Ihrem jetzigen Betrieb einen beruflichen Aufstieg schaffen?

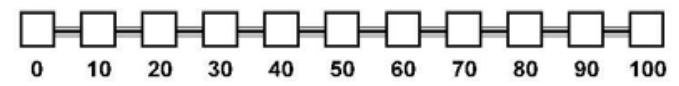

- sich selbständig machen bzw. freiberuflich tätig werden?

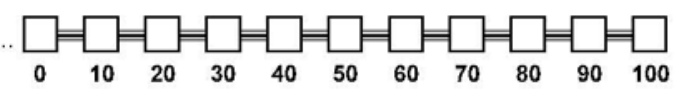

- Ihren derzeitigen Beruf aufgeben und in einem anderen Beruf neu anfangen?

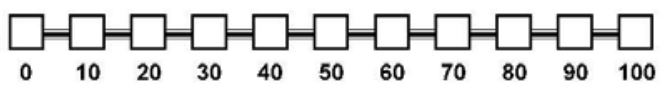

- Ihre Erwerbstätigkeit ganz oder vorübergehend aufgeben?

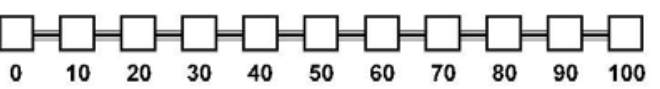

- sich in Ihrem jetzigen Betrieb beruflich verschlechtern?

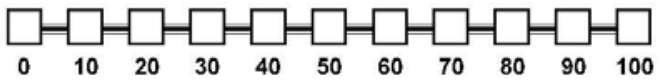

- regulär oder vorzeitig in Rente bzw. in Vorruhestand gehen?

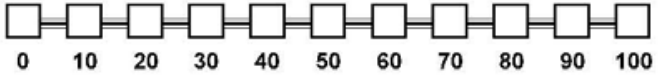

- Ihre Arbeitszeit wesentlich verändern, also von Vollzeit in Teilzeit oder von Teilzeit in Vollzeit wechseln?

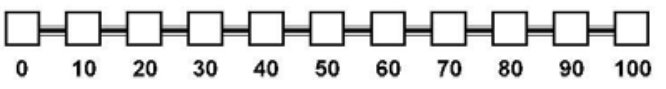

- sich durch Lehrgänge/Kurse weiterqualifizieren oder fortbilden?

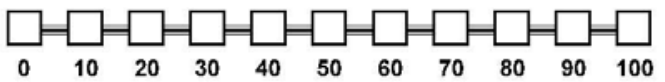

- persönlich eine Lohn- oder Gehaltserhöhung bekommen, die über die allgemeinen Tariferhöhungen hinausgeht?

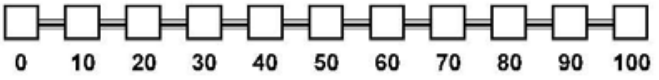


40. Wie war das in den zurückliegenden 12 Monaten in dem Betrieb, in dem Sie heute arbeiten: Hat sich die Zahl der Beschäftigten erhöht, verringert oder ist sie in etwa gleichgeblieben?

Erhöht

Verringert

Gleichgeblieben

Weiß nicht

41. Und welche Entwicklung erwarten Sie für diesen Betrieb:

Wird sich die Zahl der Beschäftigten in den nächsten 12 Monaten erhöhen,

verringern oder etwa gleichbleiben?

Erhöhen

Verringern

Gleichbleiben

Weiß nicht

42. Wenn Sie Ihre jetzige Stelle verlieren würden, wäre es für Sie dann leicht, schwierig oder praktisch unmöglich, wieder eine mindestens gleichwertige Stelle zu finden?

Leicht

Schwierig

Praktisch unmöglich

43. Liegt Ihr derzeitiger Arbeitsplatz an Ihrem Wohnort?

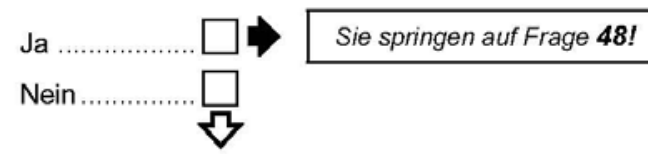

44. Wie oft pendeln Sie von Ihrer Wohnung zu Ihrem Arbeitsplatz?

Täglich

Wöchentlich

Seltener

45. Wie viele Kilometer ist Ihr Arbeitsplatz von Ihrer Wohnung entfernt?

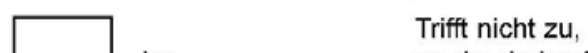

$\mathrm{km} \quad$ wechselnder Arbeitsort

46. Seit wann pendeln Sie schon von Ihrer Wohnung zu einem Arbeitsplatz außerhalb lhres Wohnorts?

Seit 19

47. Befindet sich Ihre Arbeitsstelle in -

einem der alten Bundesländer (inklusive Berlin West)

einem der neuen Bundesländer (inklusive Berlin Ost)

im Ausland 
48. Kommt es vor, daß Sie Ihre Beschäftigung zu Hause ausüben?

$\begin{aligned} \text { Ja ................. } \square \text { Wie häufig? } & \text { Täglich ........................................ } \\ & \text { Mehrmals in der Woche } \\ & \text { Alle } 2 \text { bis } 4 \text { Wochen ................ } \\ & \text { Seltener, nur bei Bedarf ......... }\end{aligned}$

49. Wenn es Ihr Betrieb lhnen freistellen würde, zeitweise auch zu Hause zu arbeiten, würden Sie dann dieses Angebot annehmen?
$\mathrm{Ja}$
Ist bei meiner Arbeit nicht möglich

Nein

50. Benutzen Sie beruflich einen Computer?

Gemeint sind Personal-Computer (PC) oder Großrechneranlagen

Ja ............... $\square$ und zwar: mit Internet-Anschluß

Nein

ohne Internet-Anschluß

51. Wie viele Wochenstunden beträgt Ihre verein barte Arbeitszeit ohne Überstunden?

\begin{tabular}{ll}
\hline \\
\hline
\end{tabular}, \begin{tabular}{l}
$\square$ Stunden pro Woche Keine festgelegte Arbeitszeit \\
\hline
\end{tabular}

52. Und wieviel beträgt im Durchschnitt Ihre tatsächliche Arbeitszeit einschließlich eventueller Überstunden?

\begin{tabular}{ll}
\hline \\
$\square$
\end{tabular} Stunden pro Woche

53. Sind Sie durch diese Tätigkeit sozialversichert, das heißt: zahlen Sie bzw. Ihr Arbeitgeber Beiträge zur Rentenversicherung?<smiles>[131In]CC1CCC1</smiles>

Nein

Trifft nicht zu, bin Selbständiger

54. Wenn Sie den Umfang lhrer Arbeitszeit selbst wählen könnten und dabei berücksichtigen, daß sich Ihr Verdienst entsprechend der Arbeitszeit ändern würde:

Wie viele Stunden in der Woche würden Sie dann am liebsten arbeiten?

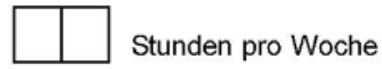

L 
55. Wie viele Tage in der Woche arbeiten Sie in der Regel?

Tage pro Woche Trifft nicht zu, da -

- Zahl der Tage nicht festgelegt ....

- wöchentlich wechselnde Zahl der Tage

56. Wie viele Stunden arbeiten Sie in der Regel pro Arbeitstag?

\begin{tabular}{|l|l}
\hline & \\
&
\end{tabular} Stunden $\quad \begin{aligned} & \text { Wechselnd, } \\
& \text { unregelmäßig }\end{aligned}$

57. Kommt es vor, daß Sie Überstunden leisten?
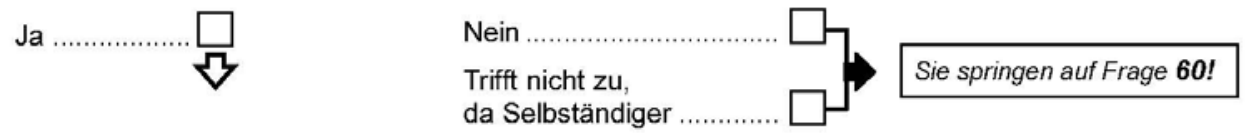

58. Wenn Sie Überstunden leisten, werden die in der Regel abgefeiert oder bezahlt oder gar nicht abgegolten?

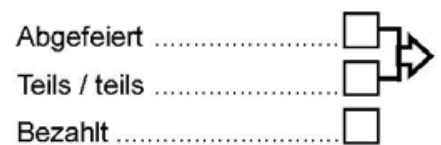

Können Sie für angefallene Überstunden auch ganze Tage Freizeit nehmen oder nur stundenweise?

Bezahlt

Auch ganze Tage

Gar nicht abgegolten

Nur stundenweise

59. Wie war das im letzten Monat:

Haben Sie da Überstunden geleistet? Wenn ja, wieviele?

Ja, und zwar:

Stunden

Nein

60. Wie hoch war Ihr Arbeitsverdienst im letzten Monat?

Wenn Sie im letzten Monat Sonderzahlungen hatten, z.B. Urlaubsgeld oder Nachzahlungen rechnen Sie diese bitte nicht mit. Entgelt far Uberstunden rechnen Sie dagegen mit.

Kindergeld bitte hier nicht mitrechnen, auch wenn es vom Arbeitgeber ausgezahlt wird.

Bitte geben Sie nach Möglichkeit beides an:

- den Bruttoverdienst, das heißt Lohn oder Gehalt vor Abzug der Steuern und Sozialversicherung

- und den Nettoverdienst, das heißt den Betrag nach Abzug von Steuern und Beiträgen zur Renten-, Arbeitslosen- und Krankenversicherung.

Der Arbeitsverdienst betrug:

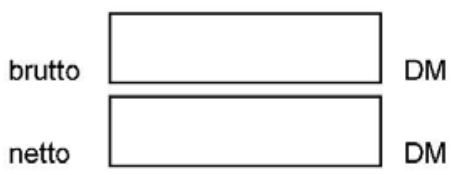


Ab hier wieder an alle!

61. Neben Beruf, Haushalt, Ausbildung oder als Rentner kann man ja noch Nebentätigkeiten ausüben. Üben Sie eine oder mehrere der folgenden Tätigkeiten aus?

Nicht gemeint ist die schon beschriebene Enwerbstätigkeit!

Mithelfender Familienangehöriger

im eigenen Betrieb

Regelmäßige Nebenerwerbstätigkeit.

Nein,

nichts davon

Gelegentliche Arbeiten gegen Entgelt

62. Was für eine Tätigkeit ist das überwiegend?

Bitte in Druckbuchstaben eintragen!

63. Welchem der folgenden Bereiche würden Sie Ihre Tätigkeit zuordnen?

Land- und Forstwirtschaft

Bau

Industrie und Handwerk

Dienstleistungen

64. Für wen arbeiten Sie da?

Ist das -

- eine Firma (bzw. mehrere Firmen)?

- ein privater Haushalt (bzw. mehrere)?

- eine öffentliche Einrichtung?

- oder sonstiges?

65. An wie vielen Tagen im Monat üben Sie durchschnittlich diese Nebentätigkeit aus?

Tage pro Monat

Und wie viele Stunden durchschnittlich an diesen Tagen?

Stunden pro Tag

L 


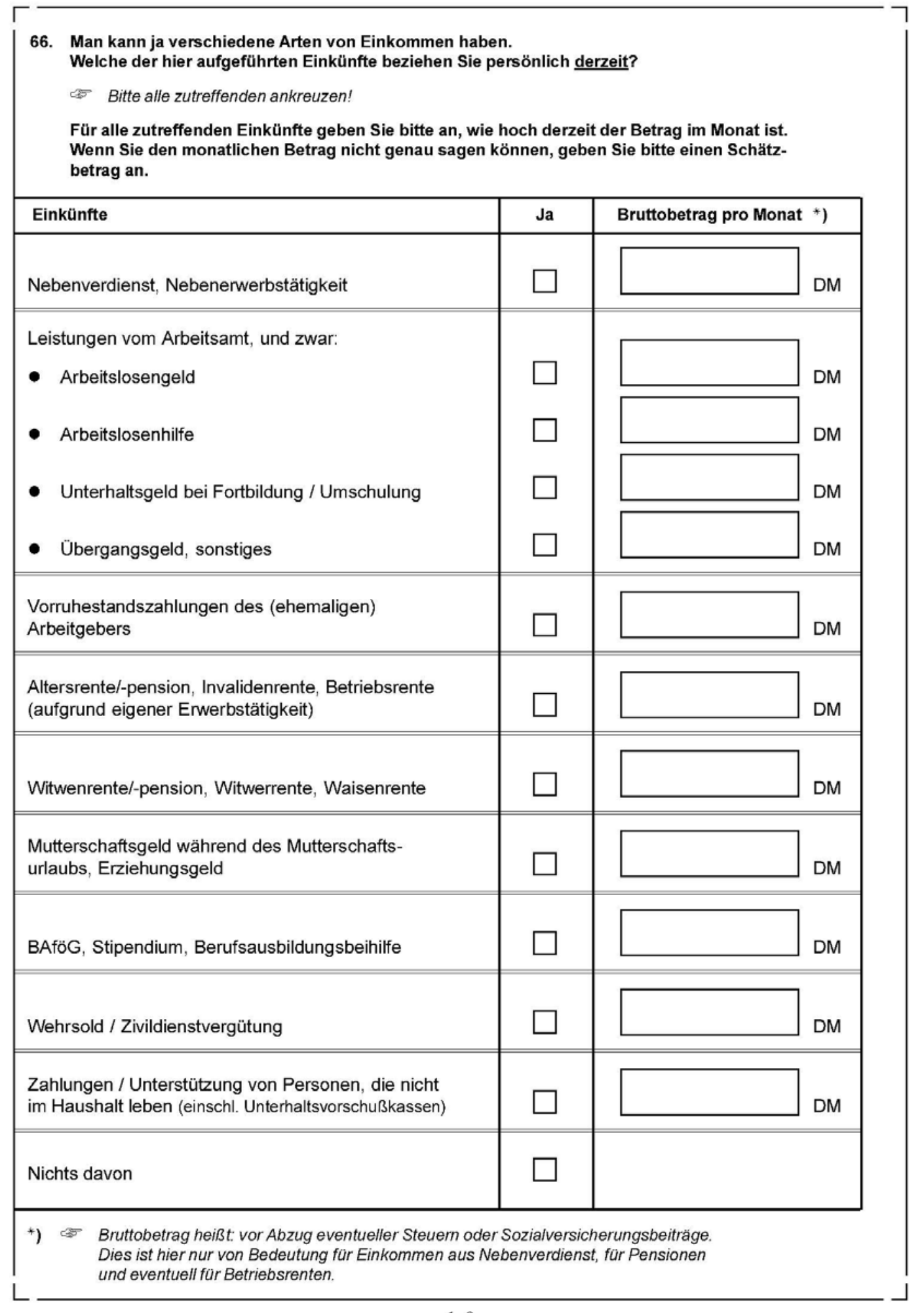




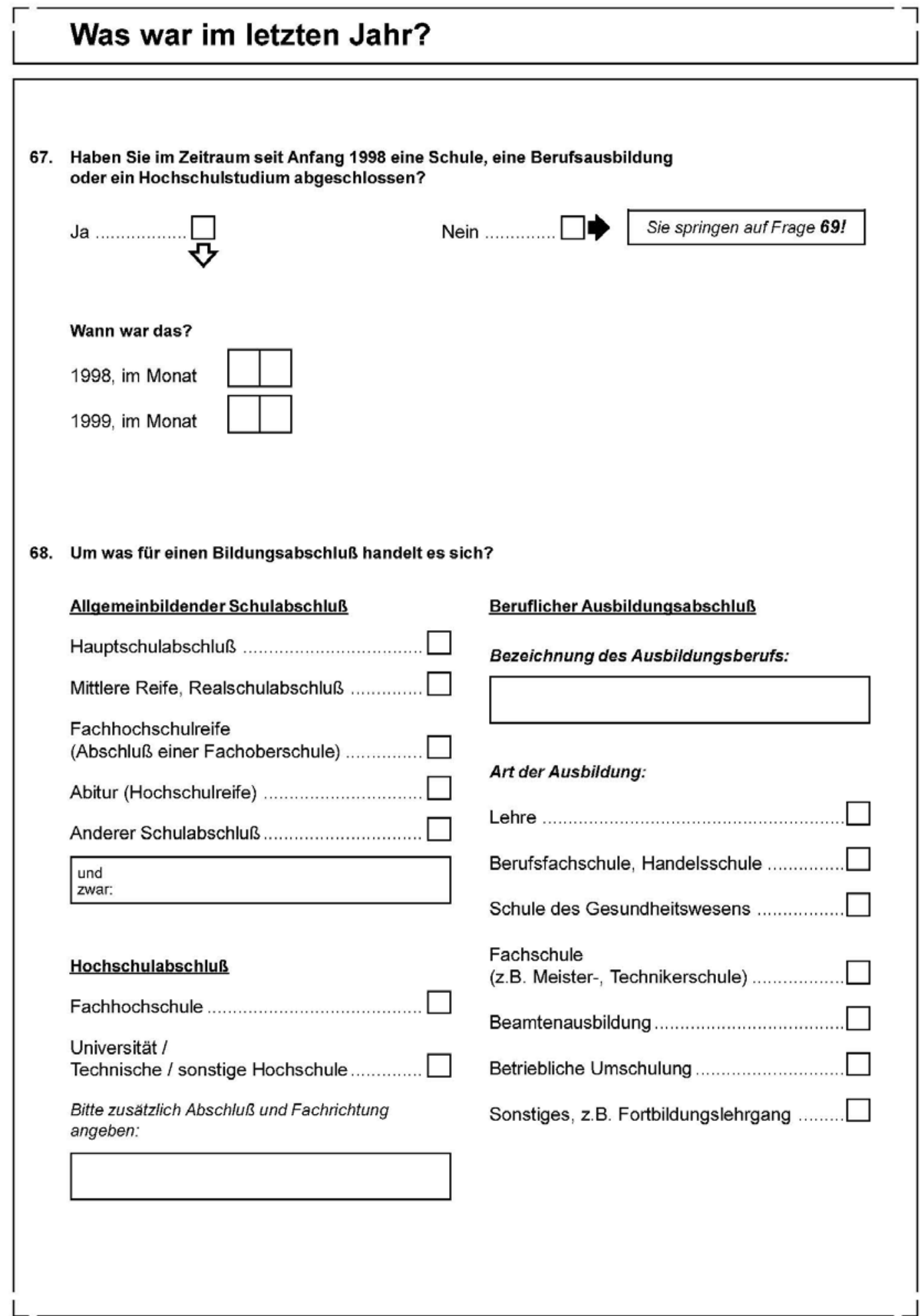


69. Sind Sie im Zeitraum seit Anfang 1998 aus einer beruflichen Tätigkeit bzw. einer Stelle, die Sie vorher gehabt haben, ausgeschieden?
$\mathrm{Ja}$
$\sqrt{2}$
Nein
Sie springen auf Frage 75 !

70. Wann haben Sie bei ihrer letzten Stelle aufgehört zu arbeiten?

1998, im Monat

1999, im Monat

71. Wie lange waren Sie insgesamt in ihrer letzten Stelle beschäftigt?

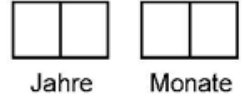

72. Auf welche Weise wurde diese Beschäftigung beendet?

Bitte nur einen Punkt ankreuzen!

Durch eigene Kündigung

Durch Kündigung von seiten des Arbeitgebers

Durch Auflösungsvertrag / im Einvernehmen

Befristete Beschäftigung oder Ausbildung war beendet

Erreichen der Altersgrenze / Rente / Pension

Beurlaubung

bei Selbständigen: Aufgabe des Geschäfts

73. Erhielten Sie vom Betrieb eine Abfindung?

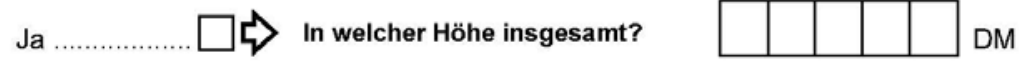

Nein

74. Falls Sie erwerbstätig sind:

Hatten Sie zum Zeitpunkt des Ausscheidens bereits eine neue Stelle in Aussicht oder bereits einen neuen Arbeitsvertrag?
Ja, neue Stelle in Aussicht
$\mathrm{Ja}$, bereits neuen Arbeitsvertrag
Nein
Trifft nicht zu,
bin nicht mehr erwerbstätig 


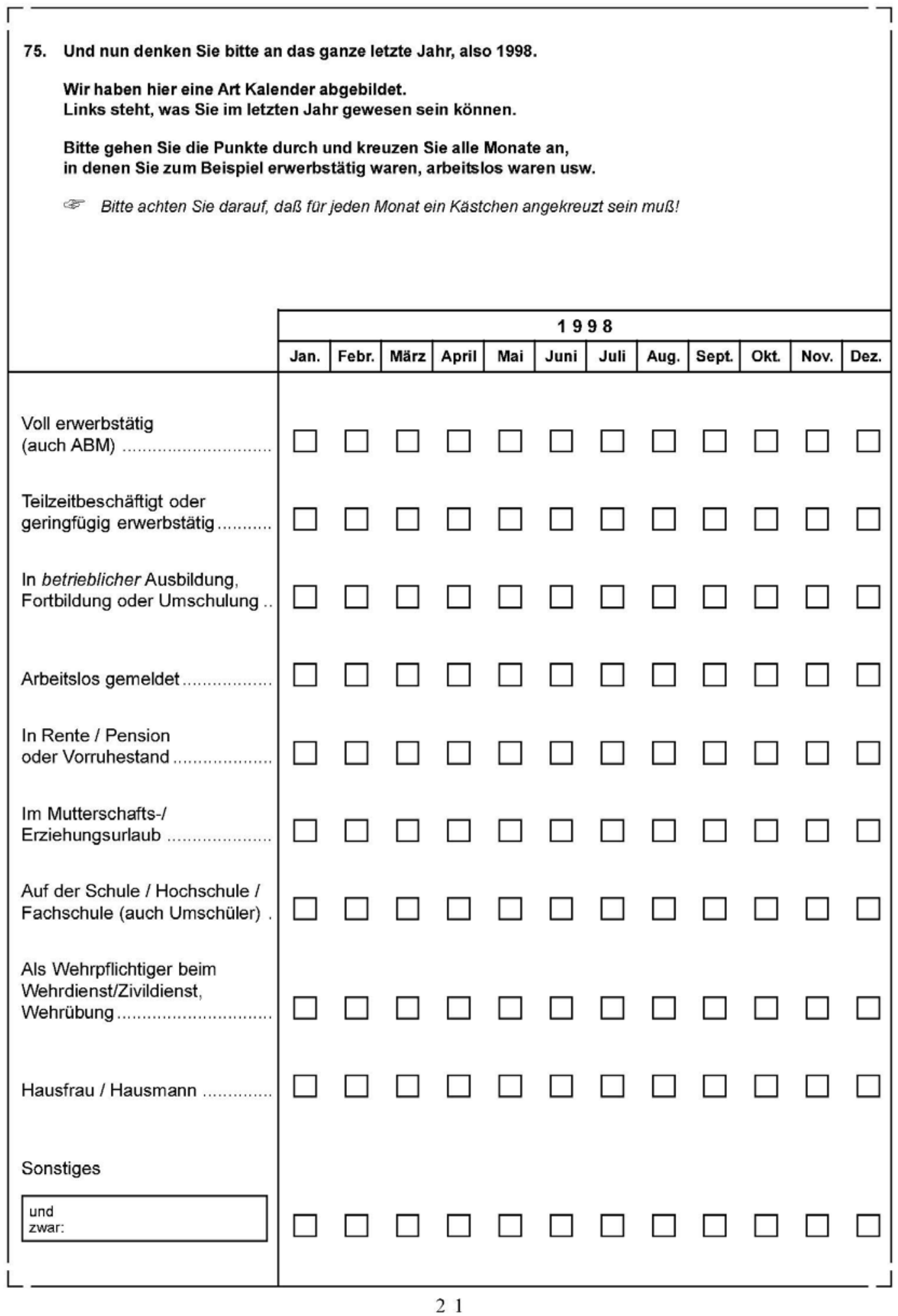




\section{Einkommen 1998}

76. Wir haben bereits nach Ihrem derzeitigen Einkommen gefragt.

Bitte geben Sie noch ergänzend an, welche Einkommensarten Sie im vergangenen Kalenderiahr 1998

bezogen haben, sei es durchgehend das ganze Jahr oder nur in einzelnen Monaten.

Sehen Sie dazu bitte die Liste der Einkünfte durch und kreuzen Sie alle zutreffenden an.

Für alle zutreffenden Einkommensarten geben Sie dann bitte an, wie viele Monate im Jahr 1998

Sie dieses Einkommen bezogen haben und wie hoch im Durchschnitt der monatliche Betrag war.

(Gemeint ist der Bruttobetrag, also vor Abzug eventueller Steuern und Sozialversicherungsbeitrăge).

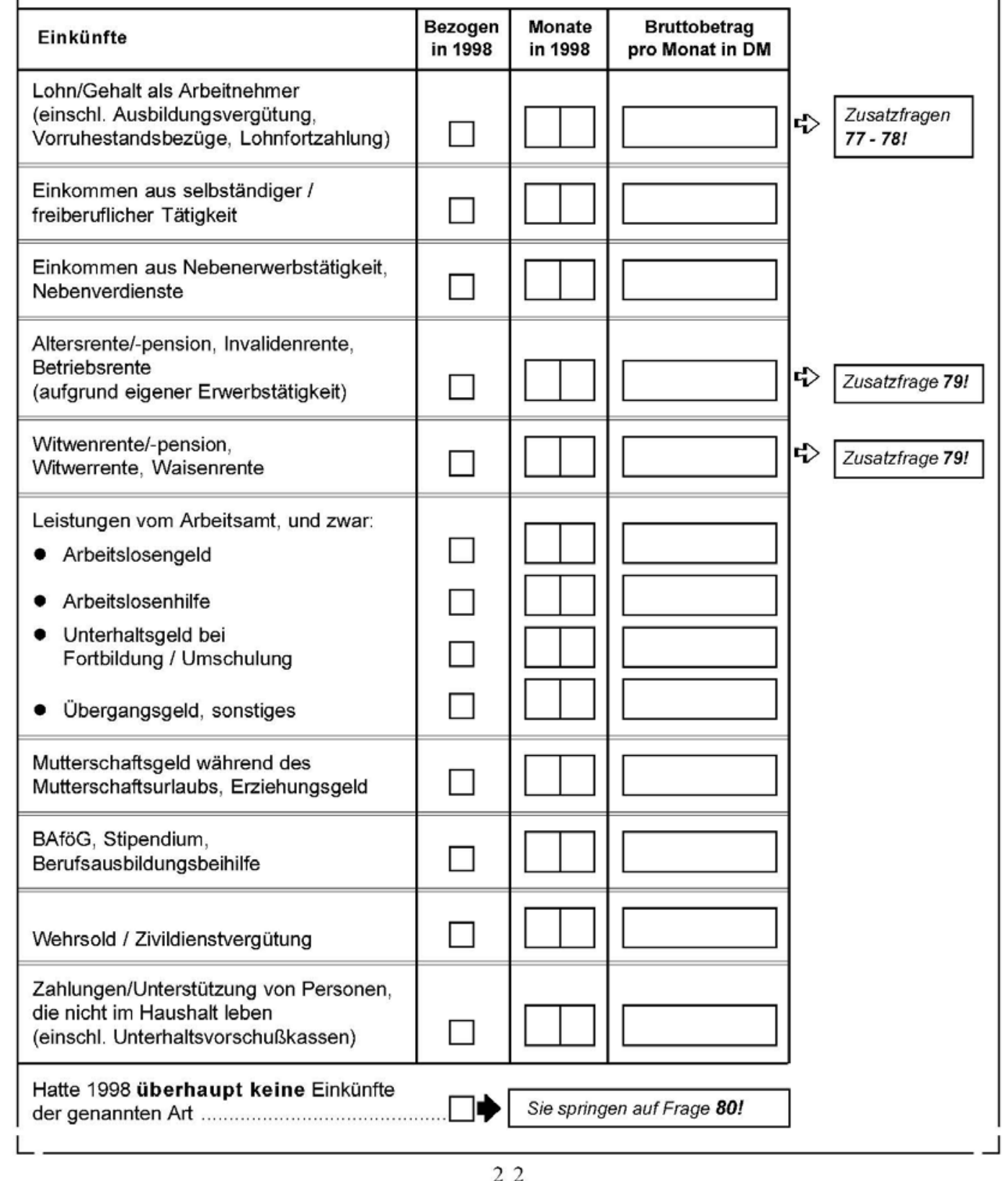




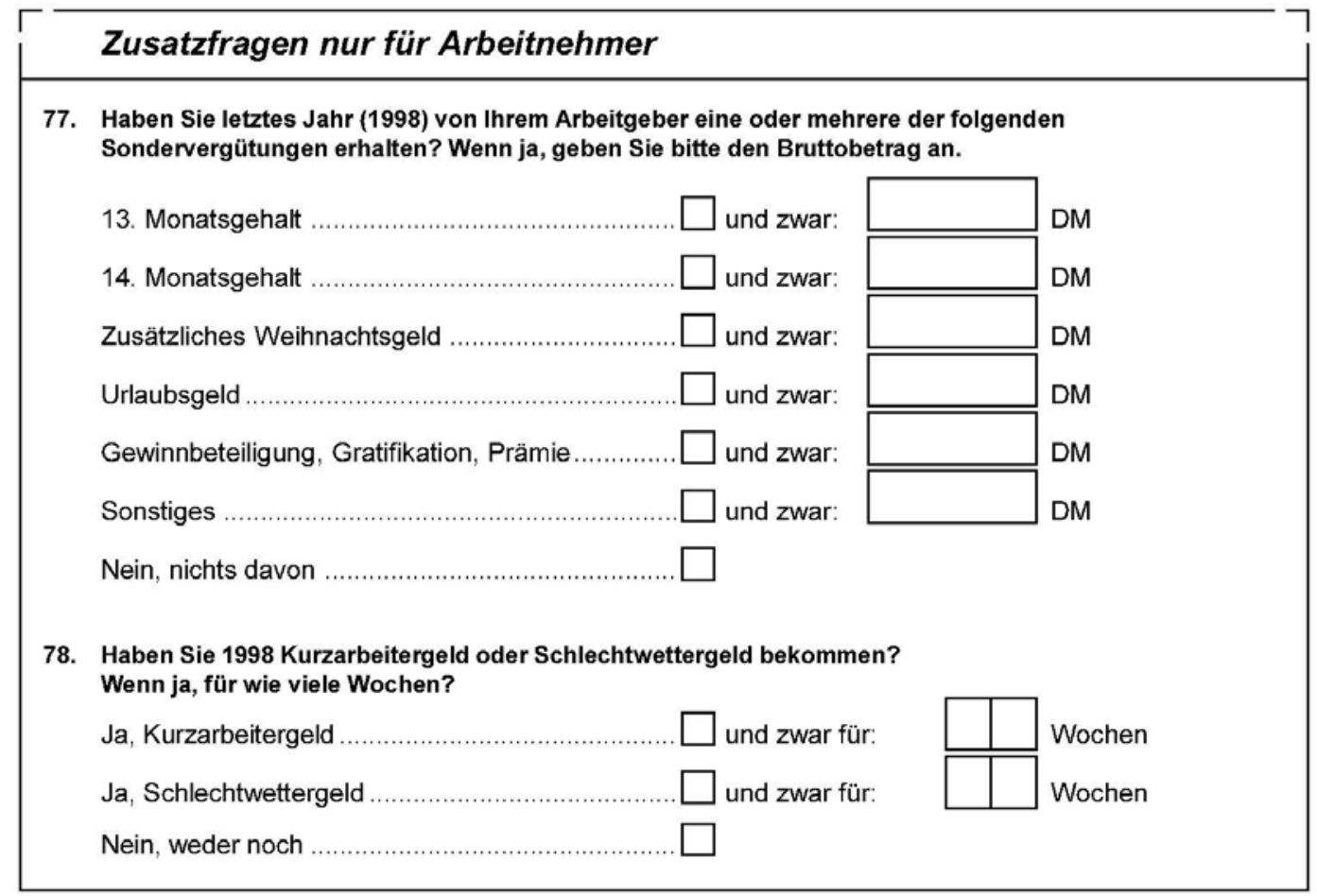

\section{Zusatzfragen nur für Rentner/Pensionäre}

79. Von wem wird die Rente $/$ Pension gezahlt und wie hoch waren die monatlichen Beträge 1998?

Geben Sie bitte den Bruttobetrag an, also vor Abzug eventueller Steuern.

Falls Sie mehrere Renten beziehen, geben Sie bitte jede zutreffende an.

In diesem Fall prufen Sie bitte auch, ob Sie in Frage 76 keine Rente vergessen haben.

Wenn Sie den Betrag nicht genau wissen, geben Sie bitte einen Schätzwert an.

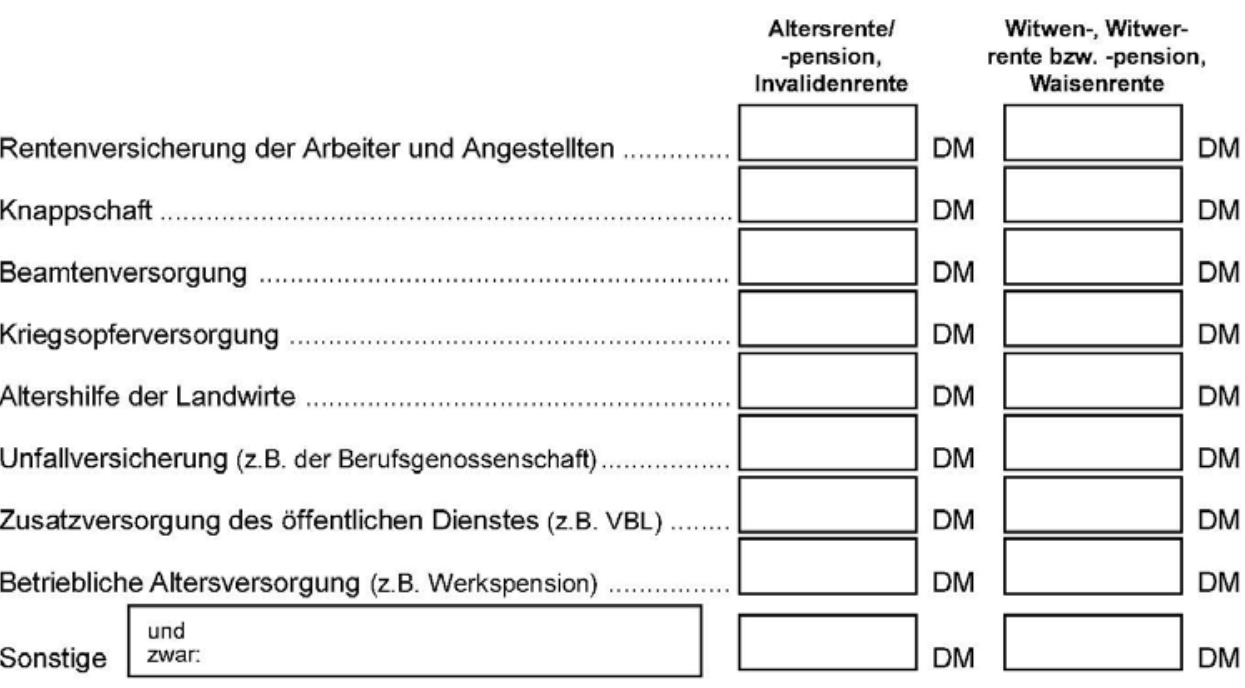




\section{Gesundheit und Krankheit}

80. Wie sind Sie krankenversichert:

Sind Sie in einer gesetzlichen Krankenversicherung (mit oder ohne privater Zusatzversicherung) oder sind Sie privat versichert oder weder - noch?

Bitte auch angeben, wenn Sie nicht selbst Beitrăge zahlen, sondern als Familienmitglied oder sonstwie mitversichert sind.

Welche Kassen zur "gesetzlichen Krankenversicherung" gehören, zeigt die folgende Frage 81

In einer gesetzlichen

Krankenversicherung

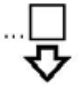

Privat versichert ..... $\square$

Sie springen auf Frage $\mathbf{8 7 !}$

81. Welche Krankenversicherung ist das?

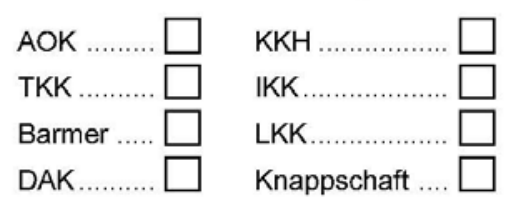

Betriebskrankenkasse ...

Sonstige

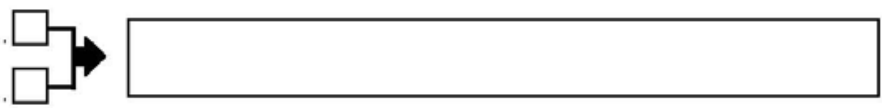

82. Sind Sie persönlich -

- beitragszahlendes Pflichtmitglied.

- beitragszahlendes freiwilliges Mitglied

- mitversichertes Familienmitglied

- als Rentner / Arbeitsloser / Student / usw. versichert? ...

83. Haben Sie eine private Zusatzkrankenversicherung?
Ja
$\frac{\square}{5}$
Nein
Sie springen auf Frage $\mathbf{8 6 !}$

84. Welchen monatlichen Beitrag zahlen Sie dafür?

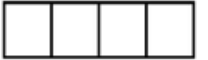

DM pro Monat

Weiß nicht .

85. Welche der folgenden Leistungen sind über die Zusatzkrankenversicherung abgedeckt?

Bitte alles Zutreffende ankreuzen!

Krankenhausbehandlung

Zahnersatz

Heil- und Hilfsmittel (z.B. Brillen)

Auslandsaufenthalt

Sonstiges

Weiß nicht . ...

86. Haben Sie Anspruch auf Beihilfe oder Heilfürsorge für Angehörige des öffentlichen Dienstes?

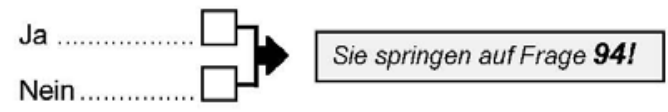




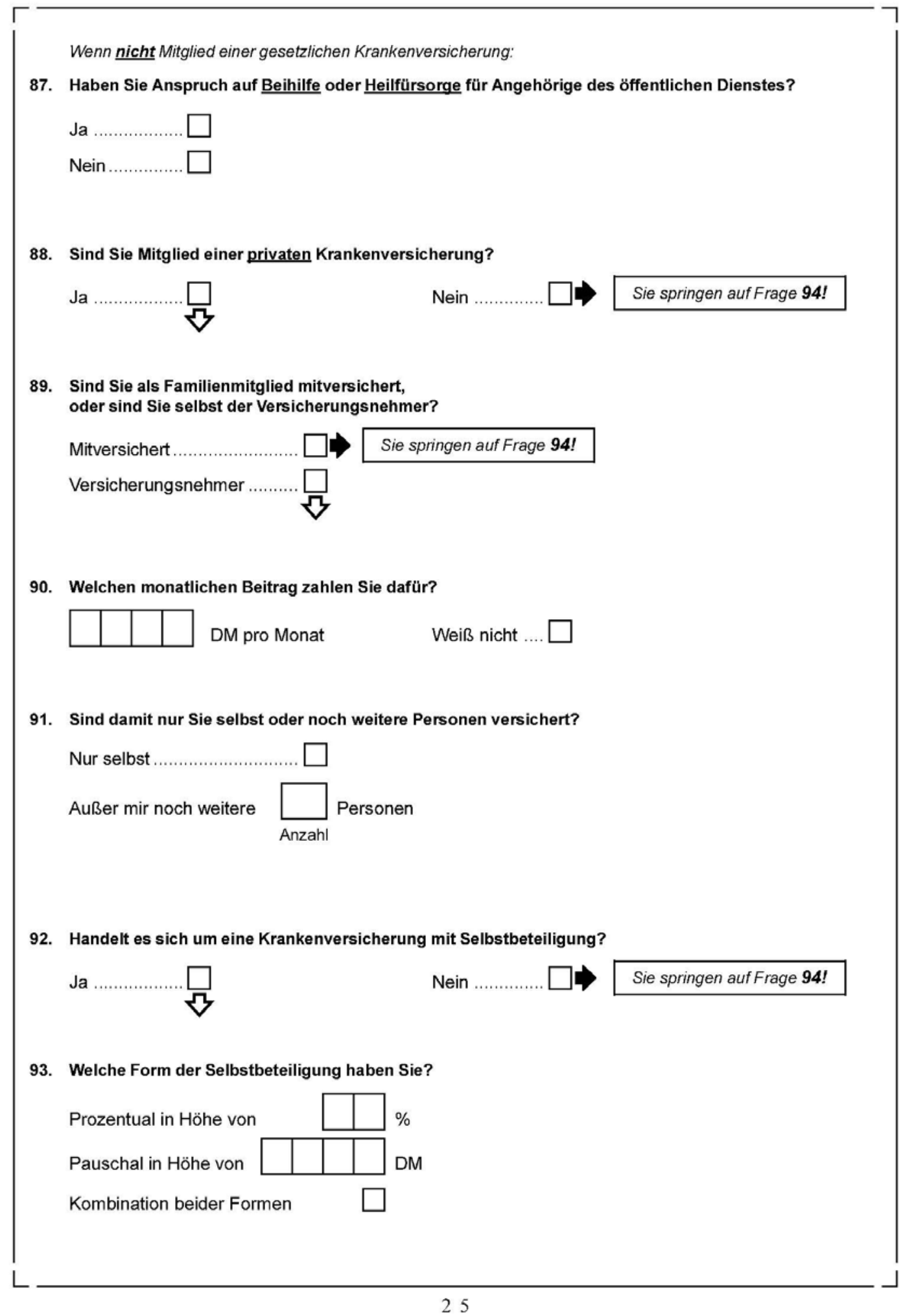


94. Haben Sie seit dem 1.1.1998 die Krankenkasse gewechselt?

Ja ........

Nein

95. Wie würden Sie Ihren gegenwärtigen Gesundheitszustand beschreiben?

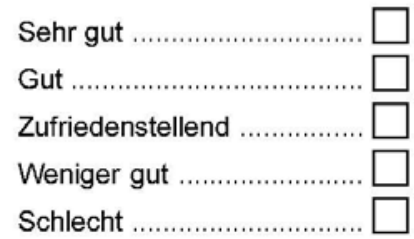

96. Von kurzen Erkrankungen einmal abgesehen. Behindert Sie Ihr Gesundheitszustand bei der Erfüllung alltäglicher Aufgaben, z.B. Haushalt, Beruf oder Ausbildung? In welchem Umfang?

Überhaupt nicht..

Ein wenig

Stark.

97. Sind Sie nach amtlicher Feststellung erwerbsgemindert oder schwerbehindert?

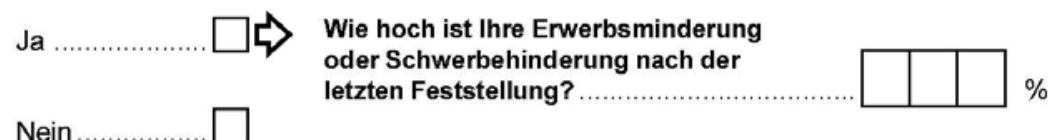

Nein oder Schwerbehinderung nach der oder Schwerbehinder
letzten Feststellung?

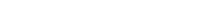

98. Haben Sie in den letzten 3 Monaten Ärzte aufgesucht? Wenn ja, geben Sie bitte an wie häufig.

Zahl aller Arztbesuche in den letzten 3 Monaten

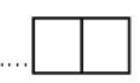

Keinen Arzt

in Anspruch genommen

99. Gehen Sie auch zu Ärzten, Therapeuten oder Heilpraktikern, deren Leistungen Sie ganz aus eigener Tasche zahlen müssen?

$\mathrm{Ja}$, regelmäßig

Nein........ 
100. Und wie war das mit Krankenhausaufenthalten im letzten Jahr?

Wurden Sie 1998 einmal oder mehrmals für mindestens eine Nacht im Krankenhaus aufgenommen?

$\mathrm{Ja}$

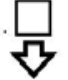

Nein

Sie springen auf Frage 102!

101. Wie oft mußten Sie 1998 für mindestens eine Nacht ins Krankenhaus?

mal

Und wie viele Nächte haben Sie alles in allem letztes Jahr im Krankenhaus verbracht?

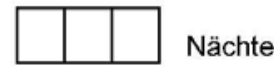

102. Falls Sie im Jahr 1998 erwerbstätig waren:

Kam es im letzten Jahr vor, daß Sie länger als 6 Wochen ununterbrochen krankgemeldet waren?

Ja, einmal.

Ja, mehrmals

Nein

Trifft nicht zu,

war nicht erwerbstätig

Sie springen auf Frage 105!

103. Wie viele Tage haben Sie im Jahr 1998 wegen Krankheit nicht gearbeitet?

Geben Sie bitte alle Tage an, nicht nur die, für die Sie eine ärztliche Arbeitsunfăhigkeitsbescheinigung erhalten haben.

Keinen Tag

Insgesamt

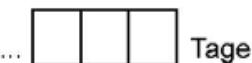

104. Waren Sie im vergangenen Jahr, also 1998, aufgrund eines Arbeitsunfalles in ärztlicher Behandlung oder im Krankenhaus?

$\mathrm{Ja}$, in ärztlicher Behandlung ......

Ja, im Krankenhaus

Nein

105. Waren Sie im Jahr 1998 zu einer Kur oder stationären Heilbehandlung?

Ja

Nein

106. Rauchen Sie?

Gemeint sind Zigaretten, Pfeife oder Zigarren.

Ja

Nein, war aber früher einmal Raucher

Nein, war nie Raucher 


\section{Einstellungen und Meinungen}

\section{Nun noch etwas ganz anderes:}

Die folgenden Aussagen kennzeichnen verschiedene Einstellungen zum Leben und zur Zukunft. Bitte sagen Sie, ob Sie für sich persönlich jeweils

- voll zustimmen,

- eher zustimmen,

- eher nicht zustimmen,

- oder überhaupt nicht zustimmen.

$\begin{array}{cccc}\begin{array}{c}\text { Stimme } \\ \text { voll }\end{array} & \begin{array}{c}\text { Stimme } \\ \text { eher }\end{array} & \begin{array}{c}\text { Stimme } \\ \text { eher } \\ \text { zu }\end{array} & \begin{array}{c}\text { Stimme } \\ \text { überhaupt }\end{array} \\ \text { nicht zu } & \text { nicht zu }\end{array}$

Wie mein Leben verläuft, hängt von mir selbst $a b$.

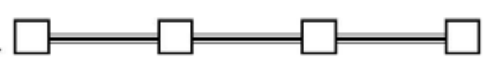

Im Vergleich mit anderen habe ich nicht das erreicht, was ich verdient hätte

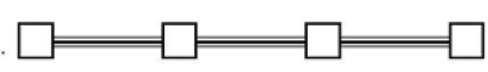

Was man im Leben erreicht, ist in erster Linie eine Frage von Schicksal oder Glück

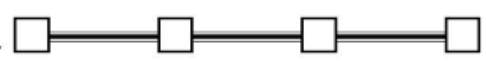

Wenn man sich sozial oder politisch engagiert,

kann man die sozialen Verhältnisse beeinflussen

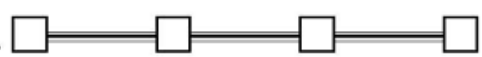

Ich mache häufig die Erfahrung, daß andere über mein Leben bestimmen

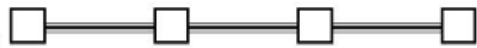

Erfolg muß man sich hart erarbeiten

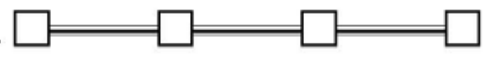

Wenn ich im Leben auf Schwierigkeiten stoße,

zweifle ich oft an meinen Fähigkeiten

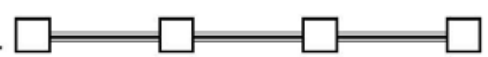

Welche Möglichkeiten ich im Leben habe,

wird von den sozialen Umständen bestimmt

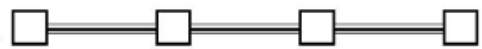

Wichtiger als alle Anstrengungen sind die

Fähigkeiten, die man mitbringt.

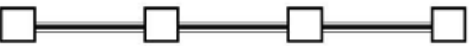

Ich habe wenig Kontrolle über die Dinge,

die in meinem Leben passieren

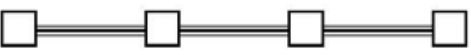




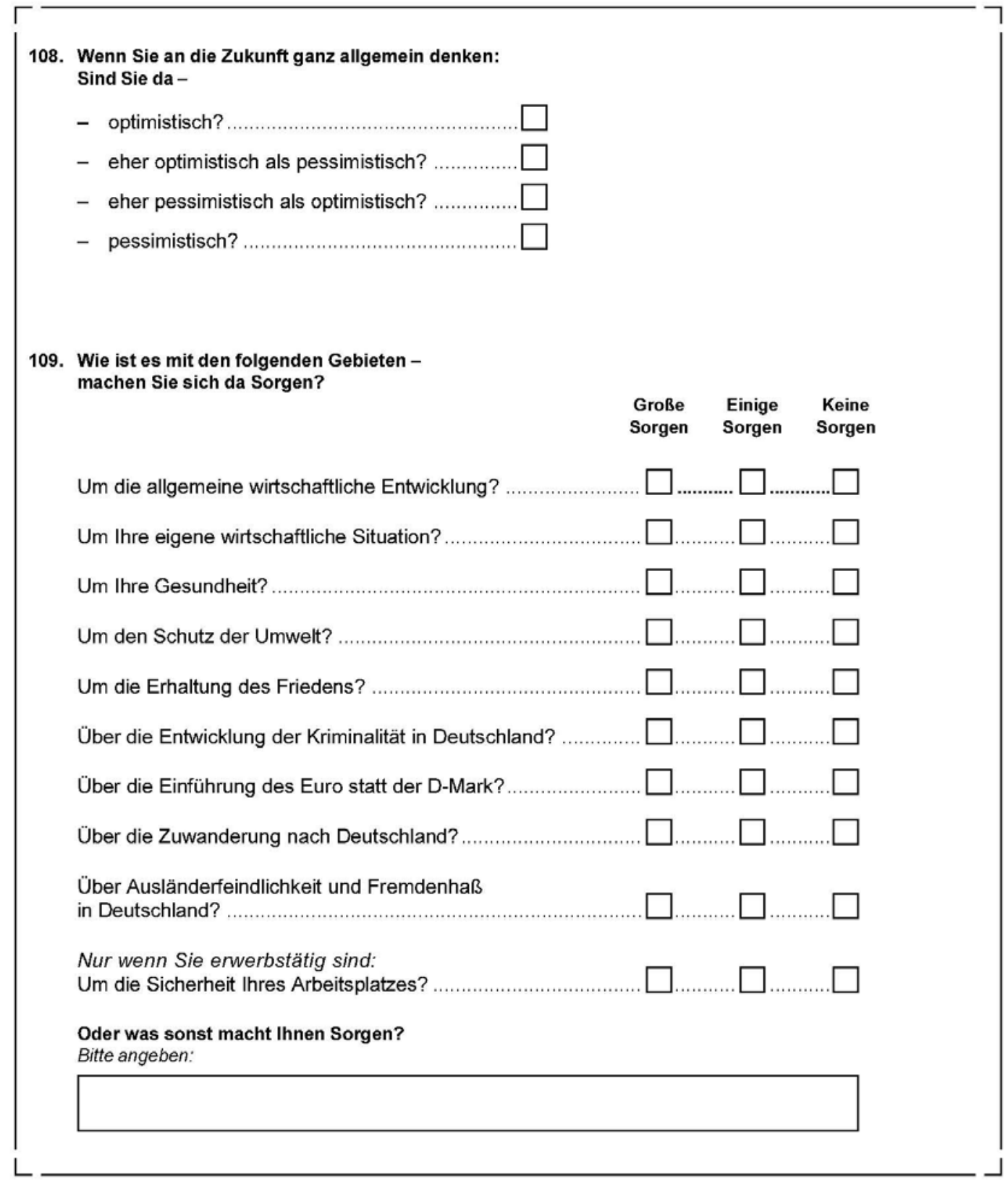


110. Einmal ganz allgemein gesprochen:

Wie stark interessieren Sie sich für Politik?

Sehr stark

Stark.

Nicht so stark

Überhaupt nicht

111. Viele Leute in der Bundesrepublik neigen längere Zeit einer bestimmten Partei zu, obwohl Sie auch ab und zu eine andere Partei wählen.

Wie ist das bei Ihnen: Neigen Sie einer bestimmten Partei in Deutschland zu?

$\mathrm{Ja}$

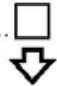

112. Welcher Partei neigen Sie zu?

SPD

CDU

CSU

FDP

Bündnis '90/Grüne

PDS

Republikaner

Andere

Und wie stark neigen Sie dieser Partei zu?

Sehr stark.

Ziemlich stark

Mäßig

Ziemlich schwach

Sehr schwach
Nein ….... $\square \quad$ Weiß nicht

Sie springen auf Frage 113! 


\section{Bindungen an diesen Ort, dieses Land}

113. Wie sehr fühlen Sie sich dem Ort und der Gegend hier, in der Sie wohnen, verbunden?

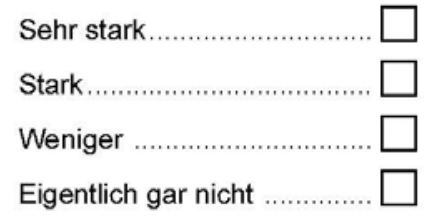

114. Könnten Sie sich vorstellen, von hier wegzuziehen, z.B. aus familiären oder beruflichen Gründen? $\mathrm{Ja}$

Kommt darauf an, will ich nicht ausschließen.

Nein, ausgeschlossen,

kaum denkbar

Sie springen auf Frage 116!

115. Befragte in den neuen Bundesländern oder Berlin-Ost:

Könnten Sie sich auch vorstellen, in den westlichen Teil Deutschlands, also die alten Bundeslănder, umzuziehen?

Befragte in den alten Bundesländern oder Berlin-West.

Könnten Sie sich auch vorstellen, in den östlichen Teil Deutschlands, also die neuen Bundesländer, umzuziehen?

Ja, gerne

Ja, unter Umständen

Eher nein.

Auf keinen Fall 
116. Haben Sie die deutsche Staatsangehörigkeit?

Ja .........

117. Sind Sie in Deutschland geboren?

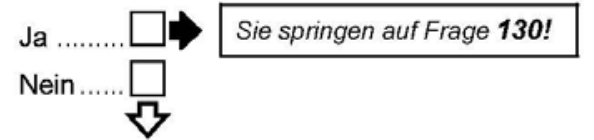

118. Haben Sie im Jahr 1984 schon in Deutschland gelebt?

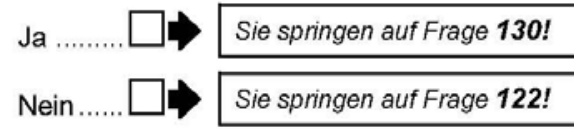

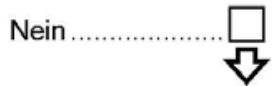

Welche Staatsangehörigkeit haben Sie?

Bitte eintragen:

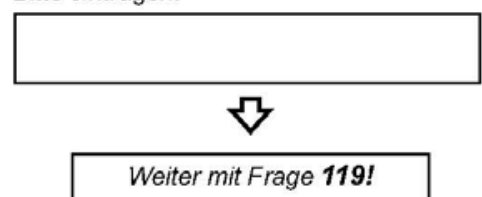

119. Hatten Sie, seitdem Sie in Deutschland sind, nähere Kontakte zu Deutschen?

Ja

Nein .

120. Haben Sie in den letzten 12 Monaten Deutsche in deren Wohnung besucht?

$\mathrm{Ja}$

Nein .

121. Wurden Sie in den letzten 12 Monaten von Deutschen in Ihrer Wohnung besucht?

Ja

Nein

122. Wenn man als Ausländer oder Aussiedler nach Deutschland kommt, hat man es nicht leicht, die deutsche Sprache zu lernen. Auf der anderen Seite: Wer lange hier lebt, kann vielleicht nicht mehr so gut die Sprache des Landes, aus dem er oder seine Familie kommt. Wie ist das bei lhnen?

Wie gut können Sie nach Ihrer eigenen Einschätzung deutsch sprechen? Und schreiben?

Sprechen Schreiben
Wie gut können Sie nach Ihrer eigenen Einschätzung noch die Sprache lhres Herkunftslandes sprechen? Und schreiben?

Sprechen Schreiben

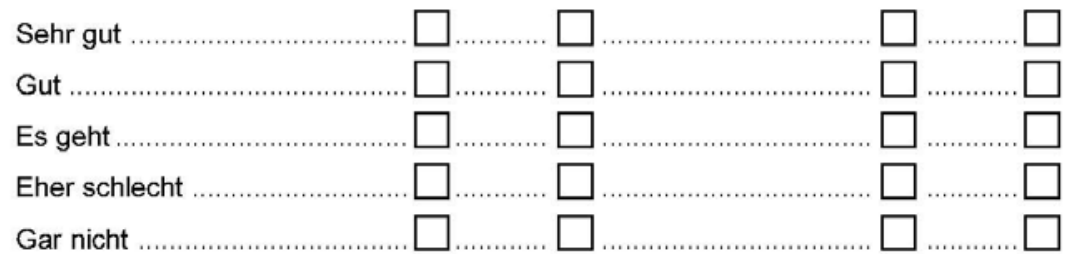

123. Sprechen Sie hier in Deutschland -

- überwiegend deutsch

- überwiegend die Sprache Ihres Herkunftslandes

- oder teils - teils?

L 
124. Wie häufig haben Sie persönlich innerhalb der letzten beiden Jahre die Erfahrung gemacht, hier in Deutschland aufgrund Ihrer Herkunft benachteiligt worden zu sein?

Häufig ............ $\square$
Selten ............ $\square$
Nie ................. $\square$

125. Möchten Sie für immer in Deutschland bleiben?

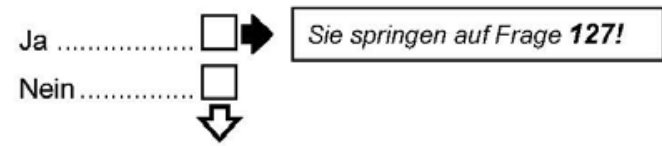

126. Wie lange wollen Sie in Deutschland bleiben?

Höchstens noch ein Jahr ......... $\square$
Noch einige Jahre ................... $\square \Leftrightarrow$ Wie viele Jahre? $\square+\square$ Jahre Weiß nicht

127. Wie sehr fühlen Sie sich als Deutscher?

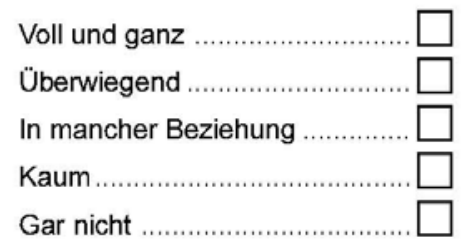

128. Und wie sehr fühlen Sie sich noch dem Land verbunden, aus dem Sie oder Ihre Familie kommen?

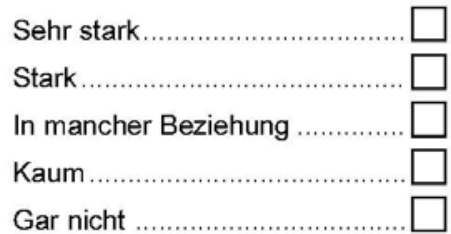

129. Haben Sie nahe Verwandte, die nicht in Deutschland leben?

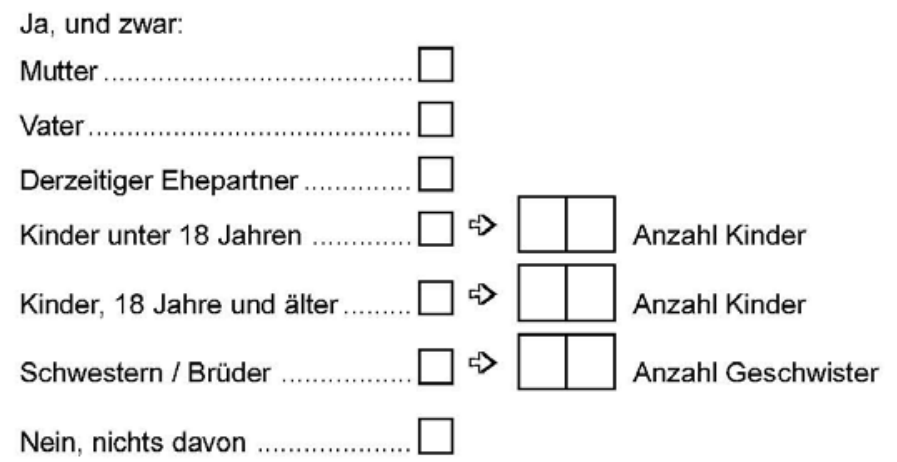


Und zum Schluß:

130. Ihr Geschlecht und Geburtsjahr:

Männlich ......... $\square$

Weiblich

Geburtsjahr: 19

131. Wie ist Ihr Familienstand?

Verheiratet, mit Ehepartner zusammenlebend

Verheiratet, dauernd getrennt lebend

Ledig

Geschieden

Verwitwet

Sie springen auf Frage 133!

132. Haben Sie derzeit eine feste Partnerschaft?

Ja

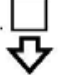

Nein

Wohnt Ihr Partner/Ihre Partnerin hier im Haushalt?

$\mathrm{Ja}$

$\square \curvearrowleft$ Bitte Vornamen eintragen:

Nein

$\square$

$\square$

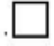




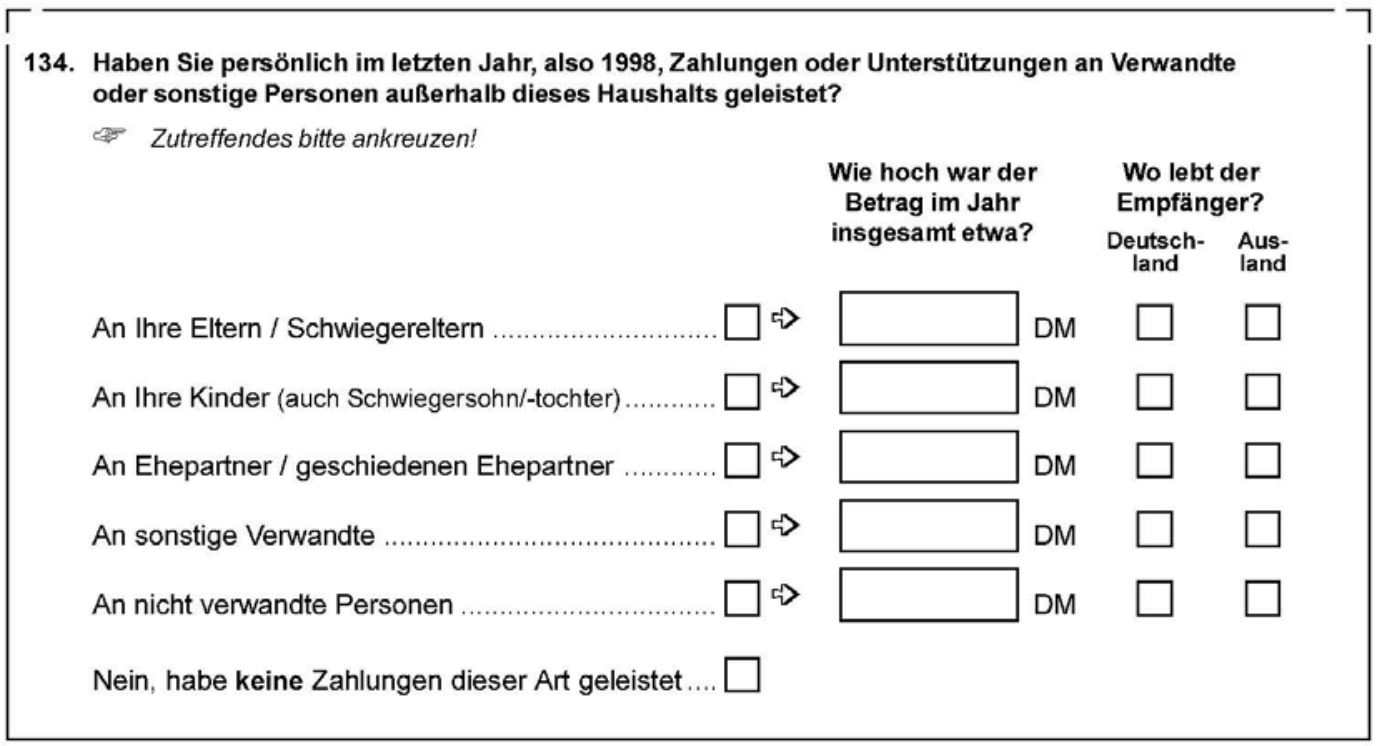

135. Zum Schluß möchten wir Sie noch nach Ihrer Zufriedenheit mit Ihrem Leben insgesamt fragen.

Antworten Sie bitte wieder anhand der folgenden Skala,

bei der " 0 " ganz und gar unzufrieden, "10" ganz und gar zufrieden bedeutet.

Wie zufrieden sind Sie gegenwärtig, alles in allem, mit Ihrem Leben?

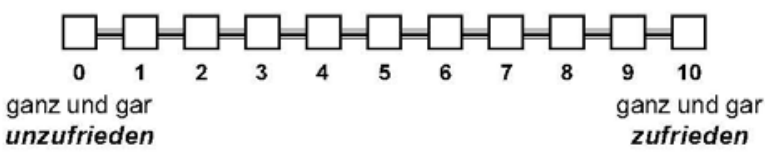

Und was glauben Sie, wie wird es wohl in fünf Jahren sein?

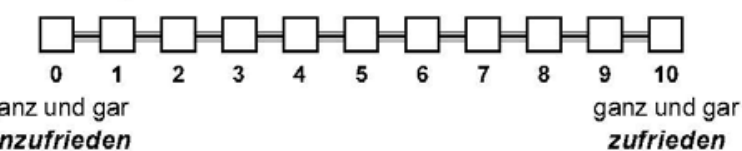

136. Wenn Sie einmal 10 Jahre zurückblicken - also auf 1989, das Jahr vor der Vereinigung Deutschlands -, was würden Sie sagen:

Hat sich seit dieser Zeit lhre Lebenszufriedenheit alles in allem eher erhöht oder eher verringert?

Erhöht

Verringert

Gleichgeblieben 
Durchführung des Interviews

A Wie wurde die Befragung durchgeführt?

Mündliches Interview

Befragter hat den Fragebogen selbst ausgefüllt, und zwar:

- in Anwesenheit des Interviewers

- in Abwesenheit des Interviewers

Teils mündlich, teils selbst ausgefüllt

B Interviewdauer:

Das mündliche (Teil-)Interview dauerte

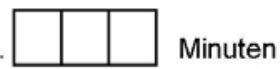

Befragter brauchte zum Selbstausfüllen

C Sonstige Hinweise:
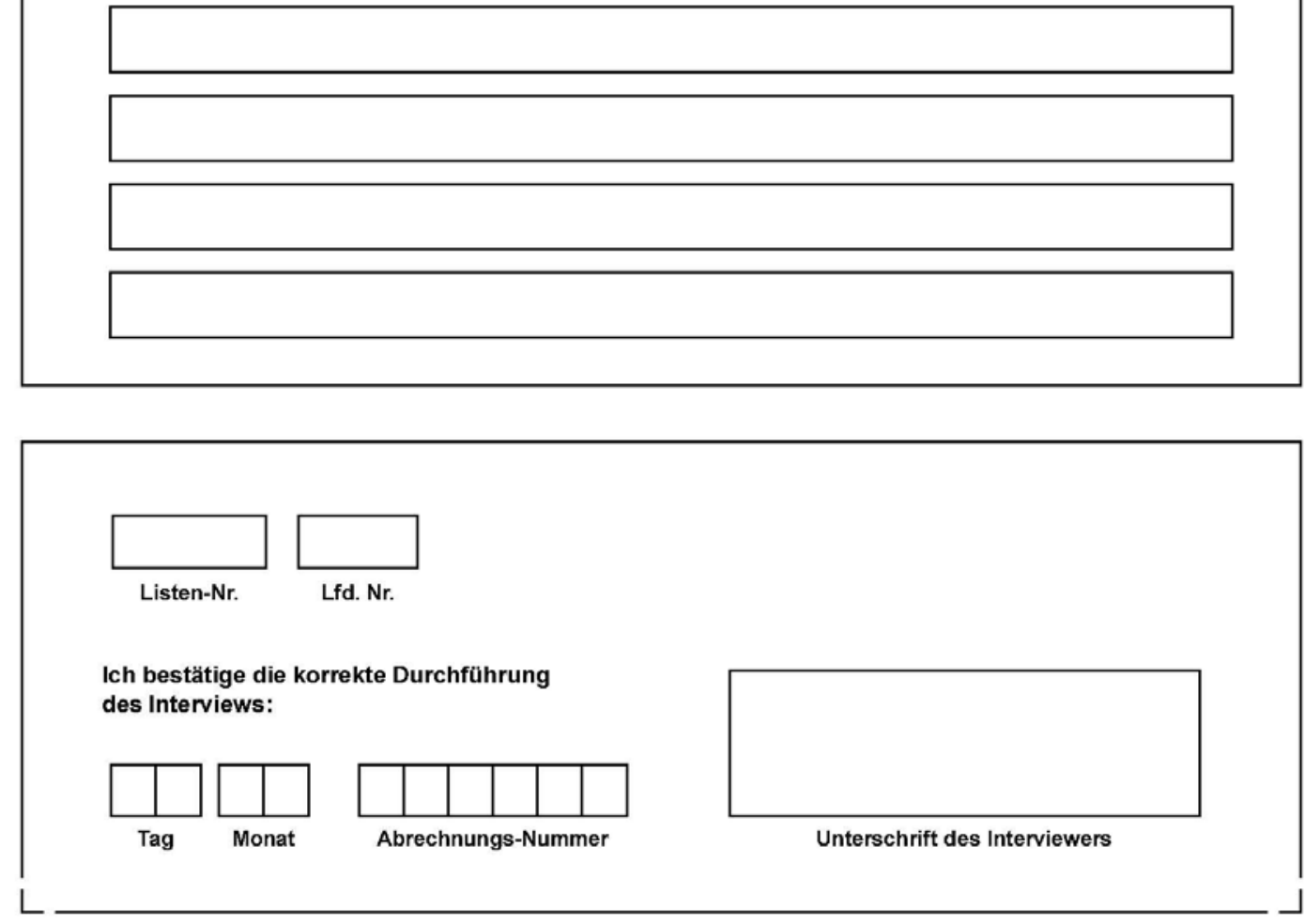


\section{Lebenslauf}

\section{Persönliche Daten Jürgen Leibold}

geboren am 15. Juli 1968

in Flieden

verheiratet, zwei Kinder

\section{Schulbildung}

Grundschule Flieden

Konrad-Adenauer-Schule Petersberg

Winfriedschule Fulda

Studium

Wirtschaftsingenieurwesen Universität-Gesamthochschule Paderborn

Volkswirtschaftslehre Justus-Liebig-Universität Giessen

Sozialwissenschaften Studienelemente: Psychologie und Rechtswissenschaften Justus-Liebig-Universität Giessen

Mitglied des 37. Studierenden Parlaments

Vordiplom

Diplomarbeit zum Thema:

„Kriminalitätsfurcht in Giessen - Untersuchung der Zusammenhänge zwischen antizipiertem Risiko, Furcht und Verhalten“

Abschluss als Dipl. Sozialwissenschaftler

Wissenschaftliche Tätigkeit

Leitung von Zenus Umfrage- und Sozialforschung

Teaching Fellow

Essex Summer School in Social Science

Data Analysis and Collection

Wissenschaftlicher Mitarbeiter am Methodenzentrum Sozialwissenschaften der Universität Göttingen

$1979-1985$

$1985-1988$

$1990-1991$

$1991-1993$

$1993-2000$

$1998-1999$

15. August 1995

13. September 2000

1997 - 1999

Juli - August 1999

seit Oktober 2000
Am Eikborn 6 37079 Göttingen juergen.leibold@gmx.de 